\title{
ACADEMIC READING \\ AND PACIFIC STUDENTS: \\ PROFILING TEXTS, TASKS \& READERS \\ IN THE FIRST YEAR OF UNIVERSITY
}

IN NEW ZEALAND

BY

MARY RUTH TOUMU'A

\begin{abstract}
A thesis
submitted to the Victoria University of Wellington

in fulfillment of the requirements for the degree of Doctor of Philosophy

in Applied Linguistics
\end{abstract}

Victoria University of Wellington

2012 



\title{
VICTORIA UNIVERSITY OF WELLINGTON
}

\author{
ABSTRACT \\ Academic Reading and Pacific Students: Profiling texts, tasks and readers in \\ the first year of university in New Zealand. \\ by Mary Ruth Toumu'a
}

Supervisors:

Dr Peter Gu

Dr John Macalister

Central to tertiary study in the literate world is the storage, transmission and retrieval of knowledge via the written word through the complex, multifaceted and largely invisible process of academic reading. With New Zealand's changing demographics and increasing participation in tertiary education by students of Pacific descent, it cannot be assumed that there is a sufficient degree of match between the cultures of academic literacy of the institution and those of the linguistic /ethnic minority student readers; and this is an issue that requires in-depth investigation. This thesis focuses on student readers of Pacific descent undertaking their first year of study in selected 100level Humanities and Commerce courses in a NZ university.

By drawing together the composite skills, cognitive and socio-cultural traditions of reading research, this study conceptualizes academic reading as the dynamic interplay between Text-Task-Reader within any given sociocultural context. This three-part understanding of academic reading enables a rich profiling of Readers, Texts, and Tasks within their contexts. It permits the systematic discovery and documentation of the nature of the challenge inherent in the academic texts and tasks of the first year of university, and 
enables the characteristics of the prototypical 'good reader' in a specific discipline to be established.

By identifying and holding the core first year academic reading Task of 'reading to understand and remember' constant, the complex interactions between the Reader and Text were able to be observed, thus providing insights into the ways in which these Pacific readers made meaning from Text. Then, through the holistic profiling of cognitive, affective, skills, and socio-culturally based reader features, the Pacific student readers' academic reading personae were constructed. Combined, the readers' profiles reveal group trends, and individually, the complete holistic profiles of two case study readers were able to be woven together from the various profiling 'strands', thus highlighting the usefulness of the profiling system and the uniqueness of the individual readers. Finally, a comparison between the 'good reader' and the 'real' student readers affords an understanding of the degree of 'fit' between the readers' characteristics and the expectations of the institution.

It is argued that this type of holistic profiling is of considerable value to institutions, enabling them to respond in informed, strategic ways to the academic literacy development requirements of their Pacific (and other) students, on both an individual and group scale. 


\section{ACKNOWLEDGMENTS}

My sincere gratitude to Victoria University of Wellington for the award of the Vice-Chancellor's Strategic Research Scholarship for 2007, which has made this research possible. I also wish to acknowledge the New Zealand Ministry of Education for the Study Award in Pasifika Education Research for 2010 which has assisted me to complete this study.

I wish to express my sincere appreciation to my supervisors Dr Peter Gu and Dr John Macalister, and to all the staff members and Pacific and other students who participated in this research and allowed me to briefly share their journeys in higher education. It has been a great privilege.

Grateful thanks also to Dr Deborah Laurs, Liz O’Connor, Laila Faisal, Jeanne and Dr Fred Biddulph for editorial assistance, support and encouragement.

Special thanks to my family, whose unfailing love, hope and faith in me inspire me each day anew. To the many others - friends, colleagues, extended family, and all who have expressed interest and support along the way - thank you.

This thesis is dedicated to my maternal grandparents Brian \& Ruth Carson who passed on during this study journey. And to my mother, Doris Carson this one's for you. 
TABLE OF CONTENTS

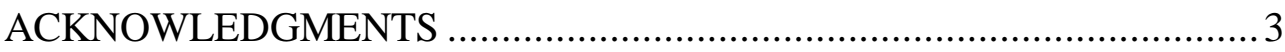

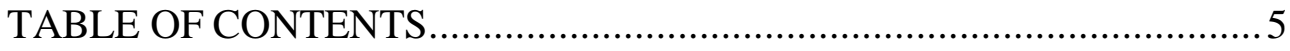

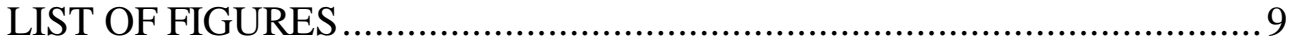

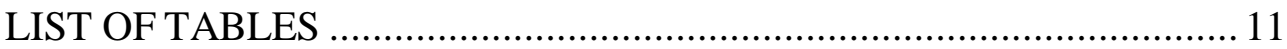

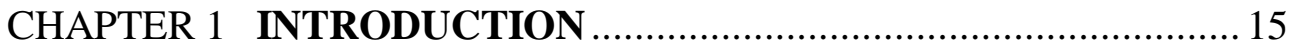

(1.1) Personal and Practical Significance of the Study, its Design and Goals . 15

(1.2) 'Pacific Students' \& Trends of Participation in Tertiary Education in New Zealand ......................................................................... 16

(1.3) Pacific Students' Achievement on Traditional Measures of Literacy at the Upper Secondary and Tertiary Levels.......................................... 18

(1.4) The Problematic Nature of Academic Reading at the Tertiary Level.22

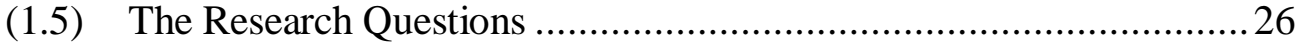

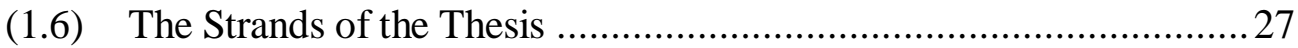

CHAPTER 2 A REVIEW OF LITERATURE RELATED TO

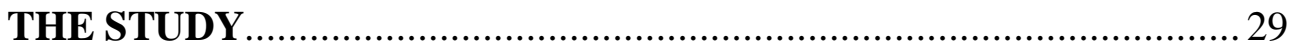

(2.1) Academic Reading in the Content Areas/Academic Disciplines: A case for the context-dependent nature of academic reading in higher education

(2.2) Academic Reading Tasks in Higher Education \& Situated Conceptions of Reading within Learning and Teaching Contexts............................32

(2.3) The Nature of Academic Texts \& the Profiling of Text 'Difficulty'.37

(2.4) The 'Reading Components' and 'Interactive-Compensatory' Perspectives of Reading................................................................. 51

(2.5) Understanding Reading as a Social Practice .....................................6 60

(2.6) Understanding Academic Readers through the Profiling of Readers \& Factors Affecting their Reading Success ........................................... 71

(2.7) The 'Good Reader' and the 'Poor reader' (and the Overarching Factor of Context)...... .94

(2.8) Linguistic/Ethnic Minority Background Students \& Readiness for Academic Reading in Higher Education. 101 
(2.9) Pacific Student Readers in New Zealand .

CHAPTER 3 THEORETICAL, CONCEPTUAL AND METHODOLOGICAL FRAMEWORKS OF THE STUDY

(3.1) Theoretical and Conceptual Frameworks of the Study

(3.2) Methodological Frameworks Designed for this Study.....

(4.1) Research Questions 136

(4.2) Research Methods: Overview and Rationale 138

(4.3) Ethical Considerations 142

(4.4) Research Setting and Participants 145

(4.5) Research Instruments and Procedures 149

CHAPTER 5 ACADEMIC TEXTS IN FIRST YEAR COURSES: FINDINGS \& DISCUSSION. 177

(5.1) Lexical Complexity of Authentic First Year Texts: Vocabulary Profiles

(5.2) Grammatical Complexity of Authentic First Year Texts: Estimates of Syntactic Complexity (SC) Based on Sentence Length

(5.3) Conceptual Density of Authentic First Year Texts: Idea Unit (IU) Density Measures.

CHAPTER 6 ACADEMIC READING TASKS: FINDINGS \& DISCUSSION

(6.1) Course Outline Content Analysis: Reading Tasks/Outcomes/Processes in First Year Case Study Courses

(6.2) Interviews with Tutors/Lecturers, and Classroom Observations ...... 207

(6.3) Therefore, the Characteristics of a 'Good Reader' 213

CHAPTER 7 THE PACIFIC READERS: FINDINGS \&

(7.1) Themes from Pacific First Year Students' Reading Histories \& Memories. 218

(7.2) Vocabulary Size Test Results for First Year Pacific and Other Students

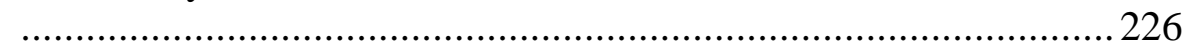

(7.3) Reading Rate Measurements of First Year Pacific Students ........... 238 
(7.4) Reported Academic Reading Strategies/Habits/Attitudes of First Year Pacific Students.

CHAPTER 8 THE READER $\leftrightarrow$ TEXT INTERACTION: FIRST YEAR PACIFIC STUDENTS' AFFECTIVE REACTIONS TO TEXTS \& PERFORMANCE IN FREE RECALL OF ACADEMIC TEXT. 255

(8.1) Pacific Readers' Perceptions of Passage Interest and Difficulty ......256

(8.2) Reader's Free Recall Performance 260

(8.3) How Course A Pacific Readers Characteristically Interacted with the Academic Texts during the Free Recall Tasks 266

(8.4) Post-task Interview Findings: What the Readers Themselves Reported about How They Read....

CHAPTER 9 THE INDIVIDUAL ACADEMIC READER PROFILES: WEAVING TOGETHER ALL THE STRANDS 283

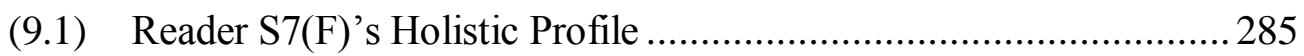

(9.2) Reader S13(PF)'s Holistic Profile .................................................... 301

CHAPTER 10 CONCLUDING THOUGHTS...................................... 317

(10.1) Matches and Mismatches between the characteristics of the first year Pacific Readers and the 'Good Reader' in Course A........................317

(10.2) Is there a link between academic reading profile characteristics and academic success?

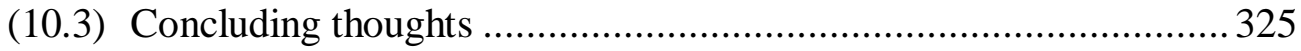

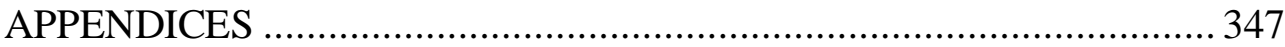

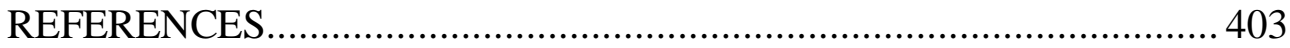




\section{LIST OF FIGURES}

Figures

page

Figure 3.1: The Reader-Text-Task conceptual framework for investigation of academic reading in higher education

Figure 3.2: Influential factors in academic reading: A framework for reader profiling

Figure 4.1: Reading strategy self-reporting scale (adapted from Zhang, 1994)

Figure 7.1: Pacific Readers' combined average estimated knoweldge of the vocabulary levels K1-K14

Figure 7.2: Pacific Readers' estimated percentage knowledge of the University Word List

Figure 7.3: Selected Course A Pacific students' estimated knowledge of vocabulary levels 1-14 \& average percentage IU recall from Course A texts

Figure 7.4: Pacific readers' self-reported use of positively framed reading strategies compared to the group average for the Pacific participant group.

Figure 7.6: Differences between Course A readers in self reported use of positively framed inventory items

Figure 7.7: Differences between Course A readers in self reported use of negatively framed inventory items

Figure 7.8: 'Most used' to 'least used' reading strategies according to readers' combined average self-ratings for positively framed items

Figure 7.9: 'Most used' to 'least used' reading strategies according to readers' combined average self-ratings for negatively framed items 
Figure 8.1: Course A Pacific readers' combined average perceived interest ratings \& percentage of IU recall for Course A passages

Figure 8.2: Course A Pacific readers' combined average perceived difficulty ratings \& percentage IU recall for Course A passages

Figure 9.1: Reader S7(F)'s vocabulary size test results compared to the average for these Course A Pacific readers

Figure 9.2: Reader S7(F)'s self-reported inventory results for positively framed items

Figure 9.3: Reader S7(F)'s self-reported inventory results for negatively framed items

Figure 9.4: Reader S13(PF)'s vocabulary size test results compared to the average for these Course A Pacific readers

Figure 9.5: Reader S13(PF)'s self-reported inventory results for positively framed items

Figure 9.6: Reader S13(PF)'s self-reported inventory results for negatively framed items 


\section{LIST OF TABLES}

Table

Table 3.1: Meyer's (2003) reader, text, task and strategy variables.....

Table 3.2: Framework for the investigation of the linguistic and non-linguistic

features of text

Table 4.1: The Pacific student reader details 148

Table 4.2: The case study courses involved in the study

Table 4.3: Sample extract from text analysis table: Dividing text into sentences . 154

Table 4.4: Sample extract from text analysis: Analysis of the sentences into IU .. 154

Table 4.5: Sample text profile summary table

Table 4.6: Sample extract from task analysis: Course outline analysis for reading tasks.

Table 4.7: Sample extract from task analysis: Thematic analysis of shared reading tasks

Table 4.8: Sample extract from task analysis: Documenting course-specific reading tasks

Table 4.9: Sample extract from reader profiling: Reader's reading history interview summary notes.

Table 5.1: Comparison of sample text vocabulary profiles for Courses A, B and $\mathrm{C}$.

Table 5.2: Syntactic complexity of passages in Courses A, B and C

Table 5.3: Idea unit density of the passages in Courses A, B and C

Table 6.1: Core reading tasks/processes found to be overlapping in Courses A,

$\mathrm{B}$ and $\mathrm{C}$

Table 6.2: Core academic reading tasks common to case study Courses A, B and $\mathrm{C}$.

Table 6.3: Core academic reading tasks shared by two courses. 193 
Table 6.4: Core generic academic reading tasks for courses categorised into content, skills or thinking development ................................................. 194

Table 6.5: Core course-specific academic reading tasks specific to Courses A,

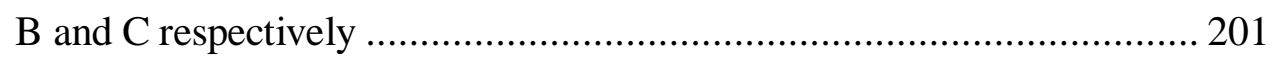

Table 6.6: Task labels for coding of lecturer/tutor interview transcript data for task analysis

Table 6.7: Task labels for coding of student readers' interview transcript data

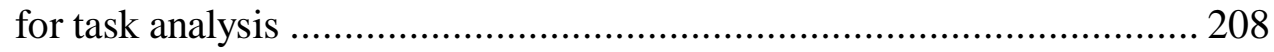

Table 6.8: Task labels for coding of classroom observation data for task analysis 208

Table 7.1: Pacific student readers' demographics and language backgrounds ...... 219

Table 7.2: Pacific student readers' combined average estimated knowledge of the vocabulary levels

Table 7.3: T-test results for comparison of vocabulary levels test results of Pacific and non-Pacific first year students..... 232

Table 7.4: Recorded reading rates in words per minute (wpm) of Pacific first year students reading authentic text samples from Courses A, B, C, D, $\mathrm{E}$ and $\mathrm{F}$...... 239

Table 7.5: Course A readers' average self-ratings for positively framed inventory items

Table 7.6: Course A readers' average self-ratings for negatively framed inventory items

Table 8.1: Course A Pacific readers' interest ratings for free recall passages....... 257

Table 8.2: Course A Pacific readers' difficulty ratings for free recall passages.... 258

Table 8.3: Course A Pacific readers' perceptive and affective responses to texts . 259

Table 8.4: Course A Pacific readers' overall amounts of IU recall........................ 260

Table 8.5: Course A Pacific readers' ranges of percentage IU recall from Free

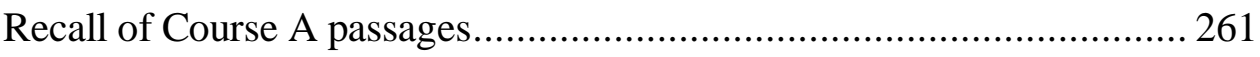


Table 8.6: Existence of IU in Course A Pacific readers' free recall transcripts

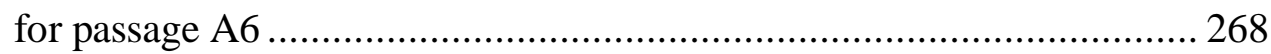

Table 8.7: Map of frequency of IU recall patterns within sentences in Course

A, passage A6. 270

Table 8.8: Textual elements not present in Pacific readers' IU free recall transcripts for passages A6-A11 in Course A

Table 8.9: Pacific readers' common meaning-making and memory-related approaches to the Course A texts during the task of 'reading to understand and remember'.....

Table 9.1: Reader S7(F)'s reading rates for Course A passages compared to Course A Pacific readers' average rates.

Table 9.2: Reader S7(F)'s prompted recall and free recall results for Course A passages.

Table 9.3: Reader S13(PF)'s reading rates for Course A passages compared to Course A Pacific readers' average rates.

Table 10.1: Comparison of the characteristics of a 'good reader' in Course A \& observations of these characteristics in the Pacific student case study readers 


\section{CHAPTER 1}

\section{INTRODUCTION}

\section{(1.1) Personal and Practical Significance of the Study, its Design and Goals}

This thesis documents the profiling of readers and their reading context, with a particular focus on readers of Pacific descent in their first year of study in a typical New Zealand university. The primary motivation for this study comes from personal experience and observations as a teacher, tutor and learning adviser at the upper secondary and foundational tertiary levels of education both in New Zealand and the Pacific. The study builds on and further explores issues uncovered in my Masters research which examined the issues relating to academic reading habits, skills and strategies, and the perceived and observed influence of participants' cultural backgrounds on their current academic reading experience.

Motivation for the research also arises from a recognition of the pressing need for inclusive and responsive strategies in tertiary education for Pacific students to enable the academic potential of Pacific peoples to be reached and used to empower individuals, families and wider communities within New Zealand. It is fuelled furthermore by the unhappy observation that there is a dearth of research and literature relating specifically to the academic reading literacy situation of Pacific students at the tertiary level in the New Zealand context upon which informed and strategic decisions can be made. 


\section{(1.2) 'Pacific Students' \& Trends of Participation in Tertiary Education in New Zealand}

The term 'Pacific student' is usually used in the New Zealand context to refer to students who self-identify as tracing their heritage to one or more of the 22 Pacific Nations within Melanesia, Micronesia and Polynesia, and are thus affiliated with one of the most ethnically and linguistically diverse regions on the planet. Similar terms such as Pasifika, Pasifiki, and Pasefika are often used to refer to this group, however the recognizable linguistic affiliations of various of these transliterations does unavoidably create exclusion, and therefore in this study the term "Pacific', in spite of all its own shortcomings, is used. Recognition of and appropriate response to this diversity is key to decision making for effecting the kinds of positive, systematic changes in tertiary education that will begin to address systemic and historic educational and literacy related inequalities that exist. Recognising the literacy-related diversity within the first year Pacific student body is integral to my thesis, and is reflected in the detailed profiling of individuals as well as the reporting of aggregate patterning.

In this study the deliberate decision was made not to take a comparative standpoint (except in the vocabulary profiling section), but rather to study these Pacific readers in and of themselves as a group of significance. The decision to focus solely on this group is for a number of important reasons. The current topical popularity of language and education-related research of and for Pacific peoples is predominantly due to the dual trends of (1) increased participation and (2) over representation in negative statistics.

In relation to the first trend, there is clear evidence of the current and likely continued growth in the size of the under 25 year old Pacific population in 
New Zealand over the coming decades. In projections of New Zealand's population in 2026

the proportion of Pasifika under-25-year-olds is expected to be 53 percent while for the total New Zealand population the expected proportion is 31 percent. As younger students tend to study with the aim of getting a qualification, it is expected that more Pasifika students will gain qualifications. (Wensvoort, 2010)

These increasing demographic and participation trends provide a strong argument for the need to recognize and respond appropriately to the widely varying characteristics of these learners.

In terms of the overall education participation rates of Pacific peoples, according to statistics published by the New Zealand Qualifications Authority (NZQA), school leavers of Pacific descent displayed the lowest rates of achievement of the qualification to enter university of all the major ethnic groupings recorded in New Zealand between 2004-2009. However despite these trends there is evidence of increasing participation by Pacific descent students in higher education. Statistics released by the Ministry of Education indicate that in 2009, Pacific student enrolment in some form of tertiary study increased by $6.2 \%$ from 2008. Figures also show that "[o]ver the 10 years from 1999 to 2009, the average annual increase in the number of enrolments by Pasifika students was 6.8 percent” (Wensvoort, 2010).

In previous years the types of fields that students of Pacific descent chose to study in and their various proportions were found to be roughly as follows (Wensvoort, 2010): 
- Management and Commerce, 29\%

- Education, $8 \%$

- Creative Arts, $7.1 \%$

- Information Technology, 4.5\%

- Society and Culture, $21 \%$

- Health, $7.2 \%$

- Engineering, 5.7\%

- Natural and Physical Sciences, $4.5 \%$

- Other, $17 \%$

Thus, the fields of Management and Commerce, and the Humanities type subjects in university such as Society and Culture and Education are the disciplines in which the largest amounts of Pacific enrolments may be found. This makes them the ideal disciplines in which to conduct initial research into Pacific descent students' reading literacy issues.

\section{(1.3) Pacific Students' Achievement on Traditional Measures of Literacy at the Upper Secondary and Tertiary Levels}

In addition to trends of growing demographics and increasing participation, the second major cause for urgency in research into Pacific peoples is their overrepresentation in negative statistics. Currently a reasonably solid research base on Pacific student literacy does exist at the pre-school, primary and secondary school levels. In the literature surveyed it became very clear that while many Pacific students do perform very well in school, according to traditional measures of reading literacy students of Pacific descent perform on average consistently lower than their counterparts at all levels of compulsory education. As a group they were found to be, on average, the lowestachieving group at primary and intermediate levels, less likely to gain a 
national qualification at the expected year level, and more likely to leave school without qualifications (Statistics New Zealand, n.d.).

Similar patterns are unfortunately also observed at the tertiary level in the limited statistics available. In fact, quite a concerning picture is painted by recent exit, retention and degree completion statistics in higher education nationwide. The current reality is that the academic potential of all our students of Pacific descent in tertiary education is simply not being fully attained or retained in our New Zealand tertiary institutions. While the causes of attrition are obviously complex, I argue that not least of these attritionrelated factors is their inability to 'crack the code' of tertiary study and their having experienced countless, repeated, negative and frustrating encounters with academic text.

However these trends are by no means unique to the Pacific student body. In most other regions globally where English is the medium of instruction in higher education, these institutions are finding that their systems are illequipped to respond to the needs of increasingly diverse students with increasingly diverse literacy and reading characteristics, backgrounds and degrees of 'reading readiness'. It follows that this is likely to also be the case in the New Zealand context. Under examination in this study is whether students of Pacific descent are 'ready' for reading in higher education and, necessarily, whether the institutions are 'ready' for them.

The problem that becomes immediately apparent is that sufficient researchbased literature on this population at the tertiary study level does not yet exist in New Zealand to give weight to the anecdotal experiences of those 'at the coal-face'. Also of concern is that while the existing figures are useful in 
allowing insight into the patterns of education generally, they do not relate specifically to academic reading literacy at the tertiary level.

In relation to reading literacy, Pacific students' performance is reasonably well documented at the lower compulsory schooling levels. In the International Association for the Evaluation of Educational Achievement (IES) Reading Literacy Study (Wagemaker, 1993), Pasifika students were found to make up the majority of the group of ESL students in New Zealand who demonstrated significant gaps in reading literacy performance. Similarly, the results of the National Educational Monitoring Project (NEMP) Reading and Speaking Assessment (Flockton \& Crooks, 2001) showed that Pasifika students in Years 4 and 8 scored lower than their mainstream counterparts. As Dickie rightly observes, "in New Zealand many students from a Pasifika background are not achieving in literacy as well as their peers and we need to seek possible causes for this" (Dickie, n.d., p.1).

A strong body of research is also forming on the related issue of adult literacy development which is often referred to in the NZ context as 'Adult Foundational Learning' and which tends to focus on the fields of language, literacy and numeracy development for adult learners. Following national trends, much of it also touches on learners of Pacific descent. Ministry of Education (2005) statistics indicate that "people who identify as Asian, Pacific, or Maori, appear to be more at risk of low English literacy", and Pacific peoples in particular. While showing a remarkably high participation rate in adult education programmes they are still the most likely to demonstrate the lowest levels of literacy in the English language. It is not surprising therefore to note that in reports on Foundation Learning in the New Zealand context, Bensemann, Sutton and Lander (2003) credit such research 
with the fact that raising the literacy levels of Pacific peoples in New Zealand is currently an overt commitment of the NZ government.

However in the face of all these negative statistics, the important challenge for researchers today is to bear all this in mind but to also actively work to no longer automatically associate this observed difference with deficit (Dickie, n.d., p.6). While the fact remains that many ESL (and other) students are at a disadvantage in the English medium institution, Mokhtari and Reichard stress that portraying them as "at risk and full of problems" (2004, p.380) is counterproductive, and they caution strongly against the "persistent search for disabling attributes" (2004, p.380).

Conceptualizing literacy as a social practice and viewing the university learning context in socio-cultural terms ideally emancipates researchers and educators, allowing them to "move beyond deficit views of individual students towards a consideration of their own teaching practices and how they could best help students to expand their literate repertoires" (Hirst, Henderson, Allan, Bode \& Kocatepe, 2004, p.66). With this knowledge of the socioculturally situated nature of reading and its componentiality, I seek to understand the academic readers and their contexts in this study, documenting both their strengths and areas for development. I do this not in order to reinforce perceptions of their unsuitability for higher education, but rather to pro-actively document their current characteristics and those of their desired discourse community, and from this point move to design ways to practically empower all involved in the learning and teaching situations to recognise and reduce disparities. 


\section{(1.4) The Problematic Nature of Academic Reading at the Tertiary Level}

In this thesis the term 'academic reading' is used to refer to reading that is carried out for academic purposes within any academic context, and it is recognized as central to the tertiary study endeavour in literate cultures. There is little doubt that in today's world, the higher education context is dominated by information, its management and dissemination. In this context, reading remains a "durable, effective and powerful means of accessing information", and given the role of books in educational contexts, the importance of students being able to "read to learn" is great. US research suggests that "approximately $85 \%$ of all college learning involves reading, and that texts are central to learning at all levels of education" (Nist \& Simpson, 2000, p.648). Thus, "[s]uccess at university depends to a large extent on students' ability to read expository texts efficiently" and "the ability to access and understand print information independently and effectively is an important characteristic of being literate at tertiary level" (Pretorius, 2006, p.433).

Moreover, this situation is not only limited to the Western or 'developed' world, since as Pretorius (2000, p.6) points out, "even in developing countries, textbooks, particularly expository texts, constitute the main medium whereby new information and knowledge is acquired, especially in the more advanced years of study. It is only through reading that one can independently access these knowledge bases", and this independent ability is crucial to and highly valued by the Western institution of higher education. Yet few would probably argue that there are still a great many students in higher education who read neither effectively nor efficiently, and this is a great cause for concern. For this reason, it is vitally important that core academic reading abilities suited to higher education be developed either prior to or early on in the tertiary study journey. 
However central to this thesis is the argument that despite the crucial centrality of reading to tertiary education, it is not as much of a concern as it should be, and this is for a variety of reasons. In an article on supporting English language learners in academic reading, Brown (2007) points out that while reading is essential to academic success, it does not receive much attention in research or literature. It has been suggested that this is perhaps due to the 'invisibility' of reading in higher education. As Block (1992) observed, reading is "such a hidden process that it often goes unnoticed in the language classroom". I would point out that if it is hidden in the 'language' classroom, how much more is it so in the mainstream tertiary classroom?

Dreyer and Nell (2003, p.350), whose studies document this same issue in South Africa, suggest that the invisibility of reading may be largely due to the fact that reading itself is not assessed in university, but the outcomes of reading are. In the mainstream university course it would appear that most often students are assigned reading to undertake on their own and in their own time, yet the outcomes of this largely unguided reading are then subjected to intense scrutiny in the classroom, tutorial, discussion group, essay or exam. Importantly too, Dreyer and Nel point out that at university, while it is assumed that students have the necessary reading skills and strategies, there is little evidence that students will acquire these skills unless they are explicitly taught.

Likewise, Mann (2000, p.311) argues that in academic contexts in higher education the "normal' process of reading is "disturbed" and becomes problematic to students. This is because of the fact that it must necessarily become a public process, and for students it ultimately becomes an activity "whose evaluated outcomes will - crucially - tell them something about their worth in the eyes of others" (Mann, 2000, p.297). In this way in tertiary 
institutions across the nation a hidden process occurs which is largely unguided, the outcomes of which are then subjected to disproportionately high levels of scrutiny by those with greater power than the reader/writer. In my view this raises many potentially problematic issues, not least of which is that the moral concerns of this situation do not appear to have been noticed by institutions of higher education in which this process is repeated daily as standard teaching and learning situations.

However despite the widespread oversight of reading, some have recognized the importance of observing the processes of academic reading in educational contexts. Those who do view academic reading as of considerable research importance argue that for facilitators of the learning experience, "knowledge of the components and management of this internal process is extremely important. Without it, educators must resort to designing reading programs based on intuitions and guesses about students' problems" (Block, 1986, p.463).

Flower, Stein, Ackerman, Kantz, McCormick and Peck (1990) insist that the chance to look into these hidden processes that lie behind the final written product is an extremely useful one and reveals valuable insights into the student's learning processes and their complicated and often confused encounters with academic discourse. As Block (1986, p.463) so aptly puts it, " $[\mathrm{k}]$ nowledge about the process, not just the product of reading, is needed if we are to move from head-scratching to designing programs which truly meet the needs of our students". Hence, it is necessary to collect process-oriented descriptions of what readers actually do before making decisions about what students need in the way of learning and skill development in any context. 
In order to do this, much more needs to be known about the readers, their institutions, the texts they interact with, and the reading demands placed upon them. The collection and synthesis of such information is necessary in order to be able to work towards strategic and evidence-based approaches to enhancing the text-based learning and teaching experiences that occur within that vital first year of higher education.

In discussing reading in the New Zealand situation it is important to acknowledge the internationally recognised role of New Zealand's reading researchers in the fields of emergent, primary, adult, and remedial reading. However there is a point during the course of compulsory education and the associated discourse surrounding it at which students make the move from supported 'learning to read' to independent 'reading to learn'. It is from this point onwards it appears that the research into academic reading in the New Zealand context becomes very scarce. This is problematic on many fronts, but primarily because of the vital need for evidence-based, research-, theory- and practice-informed responses to the academic literacy development needs of students.

While a variety of approaches exist throughout institutions of higher education for the development of academic literacy skills relevant to the tertiary context, from my observations these efforts are fragmented and are often more closely based on the conceived wisdom of the practitioners themselves rather than any widespread evidence base. Very often they are weighted more towards the development of academic writing rather than reading. This study seeks to provide a means for ensuring that when explicit developmental attention is necessary for academic reading in tertiary contexts, a detailed understanding of the demands of the texts and tasks and the nature of the readers within that socio-cultural context and the complex interactions between them is able to be 
created and that this can then provide the basis for strategic and appropriate responses.

This study argues the need for, and seeks to demonstrate the value of, a holistic approach to the understanding of the authentic texts, actual tasks and real Pacific readers embarking on their first year of study in New Zealand universities. It is designed to expose and respond to the implicit assumptions held by tertiary institutions about the role and nature of academic readers and reading, and the belief that everyone who can 'read' can 'read to learn' and should require no further assistance to do so. In universities in particular it appears that it is most often assumed that throughout their previous years of schooling most, if not all, students will have 'absorbed' the skills and abilities to be able to handle the academic reading texts and tasks presented to them in the first and subsequent years of higher education. I simply do not believe this is so.

\section{(1.5) The Research Questions}

Based on the inherent complexity of the construct of reading and its dynamic conceptualization as being a contemporaneously cognitive, affective, and socio-cultural process, this study required a holistic and multi-faceted approach. The three-part approach in the study's attempt to profile the academic reading situation in the first year of university study reflects the central theoretical framework constructed for understanding academic reading - reading as the dynamic interplay between texts, tasks and readers within a socio-cultural context. The research questions that guided this research centred on the investigation of these three inextricably bound factors, and were as follows: 
1) What is the nature of the typical academic texts encountered in the first year of university study?

2) What is the nature of the typical academic reading tasks required of the reader in the first year of university study?

3) What are the characteristics of Pacific students as academic readers in the first year of university study?

Based on the findings of these profiles and the systematic cross tabulation of features of the three main strands, richly detailed understandings of the readers, their context and interactions with text emerge. From this understanding of both the readers and their contexts, greater understanding of the potential matches and mismatches between them may be observed.

\section{(1.6) The Strands of the Thesis}

Due to the inherent complexity of the construct and its operationalization, the central metaphor by which this multi-faceted project was understood and undertaken was the woven Pandanus fibre mat commonly found in Pacific homes. The mat is created by the preparation of individual strands and the laborious weaving together of these strands to create a durable foundation. It is upon such foundations that many important discussions are undertaken and decisions made. Similarly, in this study the strands of the holistic reader, text and task profiles are individually prepared, and then woven together to reveal insights into academic reading in this context. The strategic value of the project lies in its ability to inform current and future decision making and planning with regard to the equity goals and objectives of not only the University studied but others in New Zealand and internationally where sizeable proportions of Pacific descent students are enrolled. 
This thesis comprises eight chapters which, although presented separately, are in reality interwoven strands within the fabric of the whole project. The first of which, Chapter 2, presents the review of relevant literature. Chapter 3 then details the theoretical frameworks at the heart of the study and their rationale. It is upon the main conceptual frameworks that the rest of the thesis is built. Chapter 4 continues the presentation of the methodology, context and participants, instruments and procedures employed in the study. Chapters 5, 6, and 7 present and discuss the findings resulting from the systematic profiling of the Texts, Tasks, and Readers respectively - the three main concepts within the central framework for understanding academic reading. Chapter 8 then moves to the investigation of the interaction between the Readers and the Texts. Chapter 9 refocuses again on Reader profiling and presents the holistic profiles of two case study Readers. Finally, in Chapter 10 I present my concluding thoughts on the degree of fit between the Pacific readers and their academic reading context in the first year of higher education in a New Zealand University. 


\section{CHAPTER 2}

\section{A REVIEW OF LITERATURE RELATED TO THE STUDY}

This chapter seeks to highlight (1) the importance of academic reading in higher education disciplines as a field of study, (2) the importance of increasing our understanding of the readers' contexts within higher education, and (3) the importance and timeliness of the study of the academic reading characteristics of Pacific students in higher education in New Zealand. This is done through a review of relevant literature in the following fields:

(2.1) Academic Reading in the Content Areas/Academic Disciplines: A case for the context-dependent nature of academic reading in higher education

(2.2) Academic Reading Tasks in Higher Education \& Situated Conceptions of Reading within Learning and Teaching Contexts

(2.3) The Nature of Academic Texts \& the Profiling of Text 'Difficulty'

(2.3.1) Traditional Measures of Text Readability \& Core Features Affecting the Readability/Accessibility/Difficulty of Academic Text

(2.3.1.1) Lexical Complexity

(2.3.1.2) Conceptual Density

(2.3.1.3) Non-linguistic Features

(2.4)The 'Reading Components' and 'Interactive-Compensatory' perspectives of reading

(2.5)The Social Construction of Literacy, Language and Reading: understanding reading as a social practice

(2.6)Understanding Academic Readers through the Profiling of Readers \& Factors Affecting their Reading Success

(2.6.1) The 'Mechanics' of Reading (specifically Reading Rate)

(2.6.2) Language Proficiency (specifically lexical knowledge)

(2.6.3) Attitudinal/Affective factors 
(2.6.4) The Knowledge, Use, Monitoring and Evaluation of Reading Strategies

(2.6.5) Background Knowledge/Schema

(2.6.6) Metacognition and Metacognitive Control

(2.6.7) Readers' Memory (specifically relating to the recall of expository text)

(2.7) The Good Reader' and the 'Poor Reader' (and the overarching factor of context)

(2.8) Linguistic/Ethnic Minority Background Students and Readiness for Academic Reading in Higher Education

(2.9) Pacific Student Readers in New Zealand

\section{(2.1) Academic Reading in the Content Areas/Academic Disciplines: A case for the context-dependent nature of academic reading in higher education}

In addition to the inherent complexity and invisibility of academic reading, there is also increasing evidence for the link between discipline and reading and the existence of specific traditions and cultures of academic reading in higher education. This study proposes initial frameworks for identifying these, and presents the findings of such an investigation of a higher education context in New Zealand. It can be argued that it is not only important to document the general situation within institutions, but it is vital that the actual reading competencies required by particular disciplines be clearly and accurately identified, and that the theoretical constructs underlying the actions of being able to "assess, evaluate, judge or interpret in each of these areas" (Carter-Wells, 1996, p.52) be determined and documented.

According to Sullivan (2002), a challenge to the study of reading in the disciplines in first or second language education is that the definitions of "literacy" tend to differ depending on the disciplinary lens through which one views it. Moreover, compiling research on academic reading in actual 
disciplines is often complicated by the differing terminology used to refer to a range of concepts and ideas, such as 'reading to learn', 'reading for academic purposes', and 'content area reading'. Although there is no single, unanimous definition of 'reading in the content areas', it can generally be understood to refer to a reader possessing the ability and skills to read, comprehend, react to, and learn from instructional materials (texts) in a specific subject area (Bean, 2000, p.630).

Like the study of reading in general, the study of 'reading in the content areas' has evolved in line with the prevailing theory of the day. Bean presents a brief synopsis of the historical background of content literature in European history, and points out that in the early 1900s content area reading was largely not explored. Only in the 1980s, when a "cognitivist ethos of research" prevailed (Bean, 2000, p.630) did serious research begin into reading in the content areas, but this was almost exclusively at the upper primary and junior secondary levels of compulsory education. This research, which was often criticized for its de-contextualised nature, then began to be influenced by social constructionism which "places the experiences and views of participants in a social context at the forefront" (Bean, 2000, p.631).

What is also apparent when surveying the existing research literature on the issue is that studies on ESL and EFL academic reading at the university level appear to be predominantly carried out in specific reading and/or second/foreign language classrooms, rather than in 'mainstream' content classes where students undertake authentic academic reading tasks. Even fewer studies appear to have a specific focus on the experiences, needs, and abilities of students in their first year of tertiary study. Fewer still focus on specific minority groups within these 'mainstream' first year of study. Yet 
these are the very places in which minority students are frequently overrepresented within negative educational statistics.

Of the few studies of academic reading within the disciplines of higher education and one of the most relevant to this thesis is that carried out by Kirkpatrick and Mulligan (1996, cited in Mann, 2000). They investigated the reading requirements and attitudes to reading across the disciplines of Engineering, Health Science and Social Science. Kirkpatrick and Mulligan concluded that there are significant differences in what are believed to be "appropriate reading practices" at undergraduate levels across the various disciplinary areas. Francis and Hallam (2000, p.280) also conclude that readers attempting to read in a discipline without such knowledge are likely to encounter difficulty.

\section{(2.2) Academic Reading Tasks in Higher Education \& Situated Conceptions of Reading within Learning and Teaching Contexts}

One of the features that most often defines a particular discipline (apart from the texts themselves) is the type of academic reading tasks that one is required/expected/directed to undertake with the core academic texts. In an international context, Brown and Campione (1996, p.121) explain that a lot of the activities labeled 'reading' in senior secondary levels of compulsory education are in fact "indistinguishable from critical thinking and studying". Reading in higher education has often been posited as comprising two distinct reading competencies: (1) literal comprehension skills and (2) critical comprehension skills. These differ from the reading tasks, processes and outcomes required of readers in previous and compulsory educational levels in some important ways. The existence of this 'gap' between the demands of 
compulsory and post-compulsory level academic reading is one which I believe also requires considerable research within the New Zealand context.

An important issue to bear in mind is that reading research has traditionally consisted of the researcher's interpretations of the reading processes required in particular contexts, but Devine (1988) stresses that when understanding the readers (SL readers in particular) the importance of the reader's own internalized models of the reading process must not be overlooked. There is convincing evidence that readers hold internalized models that they activate when they read and, furthermore, that these models "may be of critical importance in allowing them to strike a successful balance between bottom-up and top-down processing necessary for the interpretation of a text" (Abisamra, 2001, p.9). This is consistent with Gu's (2003, p.2) view that a

learning task is the end product in the learner's mind. ... Broadly speaking, this conception of the learning task includes the materials being learned (such as the genre of a piece of reading) as well as the goal the learner is trying to achieve by using these materials (such as remembering, comprehending, or using language).

Schumm and Mangrum's (1991, p.120) view is similar. "If students are to become self-actualizing learners, they must be able to analyze an academic task and then plan actions appropriate for completing it". In other words, what they believe about how reading happens, and how they think one learns from text in their discipline, will determine the types of reading strategies selected when reading for that purpose (Nist \& Holschuh, 2005). Koda also supports the view that students' strategy use or 'ways' of reading are dependent on their own understandings of (i) what reading entails and involves, and (ii) their understanding of themselves as readers (Koda, 2005, p.206). This study seeks to examine and facilitate the processes involved in students becoming 
successful academic readers, namely (i) gaining an understanding of the tasks and processes of academic reading that is more closely aligned with the understanding held implicitly or explicitly by one's discipline/s, and (ii) gaining an understanding of themselves as academic readers.

In the course of seeking to identify the influences of specific academic disciplines on what were believed to be "appropriate" academic reading practices within particular cases, situations and contexts, Mann (2000, p.297) demonstrated the important influence of the first year undergraduate student's "assumptions, expectations and purposes" for reading on their actual reading performance. In addition to this, Basham, Ray and Whalley (1993) point out that cross-cultural perspectives also have an effect on the shaping of these internalized task representations. Furthermore, Ridgeway (2003) makes the important observation that foreign students' conceptions of literacy may be so different as to penalize them, and that it is important for lecturers to be aware of both their own cultures of literacy and those of their foreign students.

In a similar vein, Säljö (1982) explored the relationship between the conceptions that students hold about reading, their chosen approaches for learning through text, and the quality of their learning outcomes. Säljö found that students may hold particular conceptions of learning, and may view it either as the process of constructing new knowledge or the process of memorising existing knowledge. These conceptions in turn result in the adoption of either the belief that reading is a communicative act or that it is a deciding process through which they must identify what is important to memorise. Säljö concluded that in the educational context, the communicative aspect is all too often replaced by the external drive to read what is required of them and to search for what is important to remember. Most of these researchers advocate that "practitioners involved in academic assistance 
should help students become more sensitive to their beliefs and to the contexts in which they must read and study" (Nist \& Simpson, 2000, p.539).

The situation is further complicated by the fact that teaching staff also hold their own implicit understandings and beliefs about reading. Gebel and Schrier (2002) discuss the impact of second language teachers' beliefs and practices about reading in a second language and argue that, to truly improve the practices within any educational institution it is necessary to understand what teachers believe about language learning, and how they translate these beliefs into their everyday teaching. They cite Kamil and Pearson (1979) who noted that every teacher operates with at least an implicit model of reading and furthermore, these beliefs may differ from one educational or national culture to another.

Similarly, Basham, Ray and Whalley (1993) write that whenever teaching staff ask students to perform a reading task (particularly tasks which require a written product), they already possess "underlying assumptions" about academic discourse, texts and the literate practices one undertakes with them in academia. Green (2006, p.283) found that within their own disciplines lecturers, whether they realise it or not, operate to encode "diverse combinations of socio-political, cultural, educational and ideological mores", and these principles "have a profound impact on students and their developing subject constructs" (Green, 2006, p.283). This is particularly problematic for those students whose backgrounds have equipped them with "socio-political, cultural, educational and ideological mores" that differ from those of the lecturer and/or the institution.

Adding to the complexity is evidence that the expectations placed upon student readers in many situations in higher education are not usually made 
explicit, but even when they are it does not necessarily follow that the stated expectations will be interpreted in exactly the same way by all students. Instead, there is considerable evidence to suggest that the opposite is true, and that " $[\mathrm{w}]$ hat students believe about learning and studying has an influence on how they interpret the task, how they interact with text, and, ultimately, the strategies they select" (Nist \& Simpson, 2000, p.530). Trigwell and Ashwin's (2006, p.244) research also indicated that students' conceptions of knowledge, learning, learning from text, and their conceptions of the academic tasks will to a large extent define the nature of the task for them and therefore the way that they approach that task. Similarly, Gu (2003, p.2) observed that "[d]ifferent types of task materials, task purposes, and tasks at various difficulty levels demand different learner strategies".

The degree of conscious awareness a reader possesses of their own 'modus operandi' in academic reading is also a factor that influences their interaction with text in academic contexts. As Nist and Simpson (2000, p.531) indicate, "it stands to reason that if students are not aware of their own beliefs, and of how those beliefs are in concert with those of their professors, they will have difficulty in both analyzing academic tasks and selecting appropriate strategies". It is for this reason that students' and staff members' beliefs, and the influence of those beliefs on their academic processes is an issue of increasing interest and importance to reading researchers in compulsory education contexts (Nist \& Simpson, 2000, p.530), and is also of tremendous importance in the context of higher education.

The value of this line of investigation is expressed well by Johns (1991, cited in Johns 1993, p.280), who states that the study of task representation allows the researcher to find out what kinds of operations, strategies and processes are useful to students to accomplish the genuine academic tasks required of them 
in university classrooms. Johns adds that although research into task representation is relatively recent, it can usefully enable researchers to (1) determine the differences between highly skilled and less skilled readers and (2) identify the particular "operations and goals that appear to be more valuable to successful academic literacy in a number of contexts". Such research could provide vital insights and knowledge for the development of academic literacy in specific contexts within higher education.

\section{(2.3) The Nature of Academic Texts \& the Profiling of Text 'Difficulty'}

Central to academic reading are the academic texts themselves, and the nature of these plays an important role in shaping the experiences of first year readers. Students entering higher education usually notice significant increases in both the quantity and difficulty of texts they are required to read. The types of texts read at university are usually written for a native speaking readership (Bouvet, 2002), and there is often little or no overt assistance provided to facilitate the transition between the shorter, less complex texts students have read in their earlier stages of learning, and the complex texts in university. The sheer volume of reading required in university study frequently leads to "generally reported concerns about workload in higher education" (Francis \& Hallam, 2000, p.296). These researchers also reported that frequent underestimating of the work that is needed to understand academic texts "may lead to vastly overloaded schedules of required course reading".

In addition to the increased volumes of expected reading, academic texts in

higher education are also significantly more complex than texts previously encountered. Pretorius (2006, p.433) states that academic texts in higher education "are conceptually dense and present multiple - often conflicting - 
viewpoints". Discourse analysis of academic texts shows that they are frequently characterised by:

1. lengthiness

2. complexity of both language and content

3. frequency of academic and technical vocabulary.

Readers of authentic academic texts in higher education need to be able to master "academic English", which Brown (2007, p.33) states is "fundamentally different from conversational language". These features pose challenges for many students on a number of levels. Brown (2007, p.34) also discusses the importance of noting and responding to the following challenging characteristics of academic texts in higher education:

- background world, cultural and historical knowledge:

textbook writers assume that their readers will share their cultural experiences and background knowledge. For many readers this is often not the case, and these readers must then rely on their limited knowledge of the language and the conventions of academic writing to make sense of such information.

- vocabulary:

the highly abstract nature of academic vocabulary used in academic texts can result in lack of, or inaccurate, comprehension and makes 'reading between the lines' very difficult.

- syntax:

the syntactic structures used within academic texts are often very complex, consisting of both considerable amounts of dependent or multiple clauses connecting many ideas within a single sentence, and uncommonly used constructions such as the passive voice. This presents difficulty for the student who is trying to extract the main ideas from supporting information.

- densely packed ideas: 
the density of ideas within sentences and their often convoluted presentation can slow down or distract readers.

- complex time lines:

In some disciplines, the presentation of events or description of matters does not always follow chronological order and thus may make it difficult for the reader to understand relationships between events or ideas within the text.

According to Fraser (1989, p.74) many students with English as a second language complain that in higher education there are too many unknown words, long and complex sentences in academic text, there is too much to read within time limits, and that in addition to these textual challenges they experience considerable difficulty in applying what is read to outside tasks. They face texts whose linguistic characteristics are inherently challenging, and struggle to carry out successful 'learning' from these texts. The matter is further compounded by the aforementioned fact that the academic reading tasks they are required to perform with these texts (and thus the skills and knowledge required to accomplish them successfully) vary from discipline to discipline. Often this coincides with both learners' and educators' lack of awareness and/or willingness to acknowledge these textual challenges. For example, White (2004, p.38), drawing upon Shaw (1999) notes that "[m]any college professors believe that college textbooks are not significantly different from general reading materials and that college students are able to read academic materials adequately".

Since authentic academic texts in higher education do pose a real but only lightly documented challenge to academic readers, it seems important to investigate this issue more closely. The focus needs to be on ascertaining the actual nature and degree of challenge presented by the texts within specific disciplines. This is an important first step towards providing the minority 
culture student with admission "through practice, analysis, apprenticeship and reflection" (Warschauer, 1997) to the discourse communities in which these texts exist.

\section{(2.3.1) Traditional Measures of Text 'Readability' \& Core Features Affecting the 'Readability'/ 'Accessibility'/ 'Difficulty' of Academic Text}

Fulcher (1997, p.497) states that "text difficulty, or text "accessibility" is an important but much neglected topic in Applied Linguistics", having seemingly fallen out of favour after the 1970s. Nevertheless, the difficulty of the textbook or set text/s in particular disciplines needs to be taken into consideration in programme design (White, 2004), and the principles of assessing the difficulty of text for readers used in other educational levels and areas do have widespread potential relevance to higher education and the task of assessing the demands of authentic texts within this context.

There are both quantitative and qualitative approaches to the assessment of what is variously called 'readability', 'understandability', 'difficulty' or 'accessibility'. With the qualitative approach, texts are assessed using detailed

qualitative methods involving either time-consuming and expensive comprehension testing methods, or the judgment of panels of literacy experts. The quantitative approach, on the other hand, is simpler but possibly less rigorous. It entails the use of readability formulae that appear to generally take into account measures of language variables which include word frequency and type, and sentence length.

There are many such formulae in existence. White (2004) and Nuttall, 1982) provide lists of several of the more well-known: the SMOG index devised by McLaughlin (1969); the Fry Readability Estimate devised by Fry (1963-1977); Gunning's (1968) 'Fog' measure based on polysyllabic words in text; the 40 
Dale-Chall (1945) formula involving sentence length and percentage of unknown words, and the Flesch formula (1951). These measures take samples from texts and measure 'readability' by examining a range of text characteristics.

However, the main weakness of many of the readability formulae lies in the underlying assumption that the difficulty of a given text lies completely within the text itself, when in fact there are many other factors that can also influence the reader's ability to understand the passage, and these create great variability between individuals (Bailin \& Grafstein, 2001). According to Portnuff Venable (2003, p.205), although such readability formulae allow a rough estimation of text difficulty, "the actual readability of a book depends on the reading skills, vocabulary, and background knowledge of each individual, as well as on aspects of text that are not measured by formulas, such as text structure, cohesiveness, and metaphor". It is still the case, however, that "most measures to evaluate readability are teacher directed and look at the text as isolated from the learner" (Schumm \& Mangrum, 1991, p.120).

Fulcher (1997, p.501) recognizes this limitation and although choosing to calculate text readability based on the number of syllables and length of sentences, also identifies a number of other key features not covered by this measure, including the following categories of factors of text that impact its accessibility for readers:

Factors relating to text:

- font size

- illustration and colour

- specialist use of lexis

- conceptual and propositional complexity

- textual organization 
- syntax.

Factors relating to the reader:

- background knowledge

- interest in the subject

- level of general education

- reading speed and strategies.

Schumm and Mangrum (1991) also observed that the features of text not usually covered by readability formulae but nonetheless important to readability began to feature in alternative measures constructed by a number of later researchers which set out to "gauge the "considerateness" or "friendliness" of text (Armbruster \& Anderson, 1981; Irwin \& Davis, 1980; Singer, 1986)". Those studies generally define 'considerateness' as the extent to which the text includes features which enable "information gathering" (Schumm \& Mangrum, 1991, p.120).

The four main areas of concern for the expert judges of text difficulty in Fulcher's study (1997, p.505) were:

- linguistic structure

- contextual structure (purpose and audience, context of use, information gaps, layout and visual support)

- conceptual structure (degree of familiar and unfamiliar text content, informational relationships, degree of abstractness, and temporal structure)

- reader-writer relationship (use of pronouns, tense and voice).

More recently, Dreher and Singer (2001) proposed a text analysis system comprising eight categories of features known to affect text 'friendliness':

- Text organization (ways the author has arranged the information) 
- Signaling (lexical signals of content organization)

- Discourse Consistency (a uniformity in the style of presenting information)

- Cohesion (grammatical elements that link information)

- Explication (the degree to which ideas are stated as opposed to being inferred)

- Conceptual Density (the rate at which new ideas or vocabulary are introduced)

- Metadiscourse (direction from the writer to the reader about how to read the text)

- Instructional devices (linguistic devices that highlight the text's own organization and purpose)

In addition to the reader features and text features outlined above, it is highly likely that a reader's task or purpose for reading, and the reader's ability to exploit textual clues and cues, are also important factors that influence how 'readable' a text is for any given reader at any given time. From corpus analysis, Fulcher concluded that the features that made the texts difficult also included "poor linguistic structure, contextual structure, conceptual structure, and unclear operationalisation of the reader-writer relationship" (1997, p.497). These are examples of the kinds of factors not typically covered by traditional readability formulae. The task of gauging text difficulty is obviously extremely complex, but to design manageable research it is often necessary to oversimplify the issue.

Fulcher (1997, p.498) describes how the readability or difficulty of text has traditionally been of interest to those "who need to establish the appropriacy of a given text for a pedagogic purpose", since "giving students reading material that is "too difficult" is damaging to the learning process, and demotivating to the student" (1997, p.498). However the important difference between 
Fulcher's and others' studies (including my own study reported in this thesis) is that such studies seek predominantly to allow teachers at compulsory schooling levels to select or adapt texts in order to suit learners, whereas in higher education the texts themselves cannot usually be selected according to 'ease of reading' or altered in any way. The value therefore, in predicting the difficulty of text in higher education is not for text selection purposes, but for informing those involved in teaching and learning situations about how to best support and develop readers and equip them with the skills to deal independently and successfully with the reality of often unfriendly/difficult texts.

Due to the complexity of the concept of 'text difficulty', Fulcher (1997, p.501) stresses that there is

\begin{abstract}
no simple linear relationship between estimated text difficulty and reader comprehension. In one piece of research, however, it is simply not possible to cope with all these variables. It is essential to concentrate on a small number of variables which affect text accessibility in any one study, and build upon findings as research progresses.
\end{abstract}

A comparison of the lists of factors found to influence text readability from a number of sources revealed several key features which appear to act as useful key indicators of a text's 'readability', difficulty, or level of challenge. These are outlined below, and are incorporated into the text analysis framework I have designed for this study and which is presented in the following chapter.

\title{
(2.3.1.1) Lexical Complexity
}

Virtually all models of reading and the findings of empirical research agree on the importance of vocabulary knowledge for reading, particularly for SL reading (Grabe, 1991). Koda (2005, p.49) also strongly reinforces the central 
role of vocabulary in reading comprehension, claiming that research has consistently shown that vocabulary knowledge correlates more highly with comprehension than other factors, and that the relationship between the two is also thought to be strongly reciprocal. A fundamental understanding relating to vocabulary and readability is that the proportion of lexical items that are unknown to a particular reader on a given page of text will influence the 'readability' of that text for that reader. The proportion or percentage of words a hypothetical reader needs to know in a text before they are able to successfully and independently comprehend that text is often said to form a 'threshold' for comprehension.

In terms of a vocabulary threshold for the comprehension of text, a number of figures have been proposed by various researchers. Nuttall (1982, p.26) believes that two or three percent, which represents roughly ten to fifteen unknown words on a page, are as many as an ESL reader can encounter and still be able to comprehend the meaning of the text. Similarly, Hu and Nation (2000) propose that "when a learner has $98 \%$ coverage of a text, adequate unassisted comprehension is possible". This estimate is consistent with that of Laufer (1989) who places the threshold at 95\%. It is also consistent with the estimated $99 \%$ word coverage established by Carver (1994) as the percentage coverage needed for native English speaking readers to read for pleasure. Similarly, Nagy and Scott (2000) also claim that the reader needs to know the meaning of 90-95 percent of the words in the text for adequate comprehension.

Not achieving this threshold coverage can have consequences. Koda points to some of the negative effects of unknown vocabulary in text, observing amongst other things, that "looking up words can be disruptive in information integration during syntactic and discourse processing" (2005, p.63). This is 
strongly resonant with the findings of previous research I have conducted with Pacific readers (Davidson-Toumu'a, 2005).

\section{(2.3.1.2) Syntactic/Grammatical Complexity}

The linguistic determinants of text complexity have been debated for some time (Koda, 2005, p.95), however the specific impact of syntax on readability was recognized very early on in second language reading research. In 1977, Nilagupta reported that the relationship between a reader's grammatical knowledge and their reading ability had already been under investigation for "several decades" (1977, p.90). Investigating the effect of text syntax on the comprehension of written English by Thai college students with English as a second language, Nilagupta identified specific syntactical structures in the text that were likely to cause difficulty for second language readers. Nilagupta's and other previous studies applied Chomsky's theory of transformational grammar to readability, and by building from the idea that all English sentences are made up of one or more simple "kernel" sentences that are then transformed in various ways, they came to the conclusion that some of these transformed forms present more difficulty for English second language readers than others. The five syntactic structures in particular found to have presented difficulty for non-native speaking readers were: "negatives, passive voice construction, embedding, deletion, and nominalization" (1977, p.92). It is likely though that the difficulty of various structures for English second language readers may be considerably influenced by the structures of their first language (L1).

Berman (1984) found that the two main syntactic features correlated with the greatest degree of second language comprehension difficulties were: "violation of structural prototypicality" (when the surface form of the sentence conflicts with the basic order of the semantic and syntactic relations between 
ideas in the sentence, for example, the passive construction), and "lack of structural transparency" or "structural opacity" (which occurs when cohesive devices such as deletion and substitution work to obscure the relationship between ideas in the sentence, for example the absence of the relative pronoun in reduced relative clauses) (2005, p.111).

However, according to Koda (2005, p.110), there is much debate about the existence of 'automatic' relationships between this syntactic complexity and ensuing reading comprehension difficulties. Kintsch and Keenan (1973, p.273) appear to base understandings of readability on aspects such as vocabulary density and rarity, and sentence length, but other important factors mentioned in related literature include lexical factors, the hierarchical relationships between ideas/propositions, and word class and function.

Operationalising the concept of syntactic complexity is difficult and according to Szmrecsányi (2004) it still remains a slippery construct despite having been studied repeatedly. Several approaches appear to be possible for obtaining indications of a text's syntactic complexity. Some involve a systematic, timeconsuming, sentence-by-sentence grammatical analysis of the text. Others apply measures based on more easily quantifiable features of text. Szmrecsányi (2004) looks in particular at three main measures: (1) the use of Node counts as a measure of sentence complexity, (2) the use of an Index of Syntactic Complexity (ISC), and (3) the use of sentence length as a proxy for syntactic complexity. The use of the Node count as a measure of sentence complexity is based on the understanding that "the more phrasal nodes a unit dominates, the more complex it is" (2004, p.1033). The use of the ISC involves a formula designed to yield a numerical score for structural complexity, based upon the number of certain high complexity indicators 
existing in the text (such as subordinating conjunctions, WH-pronouns, and certain verb forms and noun phrases).

The third approach adopts sentence length as a proxy for syntactic complexity, and Szmrecsányi argues that "length is taken to mean the number of words dominated by a syntactic unit" (in this case, the sentence), and therefore, the "unit's syntactic complexity will be directly proportional to the number of words it contains" (2004, p. 1033). In a comparison of these three measures of syntactic complexity, Szmrecsányi (2004, p.1037) found that measuring sentence length (SL) and computing an index of syntactic complexity (ISC) do an excellent job in approximating the node count (NODE), which is the structural and probably most 'real' measure of syntactic complexity at a cognitive level. Sentence length does appear to be a straightforward and satisfactory means by which the syntactic complexity of a text may be estimated, and is the approach chosen for use in this study.

However there are some criticisms of this approach. The main criticisms that Koda (2005) aims at it are that it necessarily leads to an overly quantitative approach to assessing text difficulty, and it cannot be assumed that there is an automatic relationship between the length of a word or sentence and its complexity. In fact, as Koda points out, some research suggests that the longer a word, the more affixes it may contain, and thus, the higher the chance of a reader guessing its meaning. Likewise, with sentence length, the longer the sentence the more subordination it is likely to contain, and thus, the possibility exists that with the presence of explicit connectives, longer sentences could actually assist comprehension rather than impair it.

Thus, while there is ongoing debate about whether a systematic relationship exists between syntactic features and comprehension problems, sentence 
length does offer a practical and valid means of easily estimating a text's syntactic complexity and, whether or not it directly affects comprehension, it is likely to directly affect the processing demands made on the reader's working memory. In this way it does certainly impact upon text readability and ease/difficulty (Koda, 2005, p.96).

\section{(2.3.1.3) Conceptual Density}

Expository text is the means by which information is conveyed, and this characteristically necessitates high levels of conceptual density. Conceptual density is defined by Dreher and Singer (2001, p.100) as the rate at which new ideas or vocabulary are introduced in a text, and has been touched on as a text feature affecting readers. However, having recognized its important influence on readers, the puzzle remaining for researchers is how best to operationalize this concept and what unit should be used to do so.

The concept of the Idea Unit or 'propositional unit' or some form of semantically based unit in text is not new, having been used by researchers for at least 40 years. Early work with the concept was undertaken by Meyer (1975) and van Dijk and Kintsch (1983) who proposed the semantic building block that they called the 'proposition'. Dennis and Kintsch (n.d., p.2) describe propositional analysis (Kintsch, 1974) as a particularly important level of analysis, and explain that "the term derives from logic, but has been widely used in linguistics and psychology to designate units consisting of one or more concepts that function as arguments (with specific semantic roles) and a relational term". Kintsch et al. demonstrated that conceptual density had a clear relationship with factors such as reading rate and text content recall. 
However, as explained later in the Methodology chapter, the propositional unit and various other units described in previous literature have proved unsuitable for analytical purposes in this study, and so an alternative was created. Since the concept of the idea unit forms a significant basis for my interpretation of the nature of text and the interaction between reader and text, it was necessary to create my own definition for use and calculations in this study, and these have been designed for and tailored to this project based on my interpretation of existing literature. Criticism of the concept of idea units is usually invoked in terms of the level of subjectivity required to define it and the seemingly arbitrary divisions that are created to define units, and this is acknowledged but I believe is far outweighed by the value of the unit for the purposes of understanding texts and readers.

\section{(2.3.1.4) Non-Linguistic Features}

The features discussed thus far have been linguistic in nature, but, as Bernhardt (1986) realized, it is important to acknowledge that the "physical fact of the text, with its spatial appearance on the page, requires visual apprehension: a text can be seen, must be seen...". White $(2004$, p.39) also noted that most specifically designed textbooks provide a number of visual and non-verbal learning aids, but that many students do not appear to understand the value of these aids or how to utilize them most effectively. Bernhardt (1986, p.67) found that these non-linguistic features are what readers gain a "heightened sense of categories, divisions, and orderly progression" from. Moreover, these non-linguistic patterns do differ by genre and discipline, and this is especially noticeable in scientific or technical disciplines in which it has been estimated that 30 percent of the text is usually composed of such non-verbal and graphic information (Rubens, 1986). 
Nuttall (1982, p.42) provides a very useful description of three categories of non-textual information that can serve to assist (or hinder) the reader in authentic reading tasks:

1) Graphic conventions - such as layout, punctuation, typeface (font), symbols, spacing, indentation

2) Reference Apparatus - such as titles, index, introductions, abstracts, summaries, table of contents, footnotes, glossaries, appendices, blurbs on covers, author's biographical information

3) Non-verbal information - such as maps, diagrams, illustrations, tables

These non-verbal features have been shown to work together with the verbal information in text to facilitate readers' comprehension (Nuttall, 1982, p.52). Of late, there has been considerable interest also in the effects of layout and non-linguistic aspects of online texts on readers. For example, Chaparro, Shaikh and Baker (2005) have claimed that the "readability of online text has been shown to be influenced by a number of typographical variables including text size and type, line length, density, margins, and white space". The range of effects on readers varies considerably from study to study, however common findings relate to the effects of features such as white space on the page on reader features such as reading speed and levels of comprehension. The existence of these measured and defined variables must be taken into account when assessing the levels of challenge presented or likely to be presented by a given text.

\section{(2.4) The 'Reading Components' and 'Interactive-Compensatory' Perspectives of Reading}

These understandings of academic reading, the nature of texts, what constitutes their difficulty, and indeed the whole of this research can be linked back to several key theoretical traditions in the study of reading. These are (1) 
the reading components perspective, (2) the interactive-compensatory view of reading, and (3) the acknowledgement of reading as occurring firmly within a socio-cultural context. These three lines of thought are explained briefly in this and the following section of this review of literature.

In the literature surveyed, a number of incarnations exist of the view that reading and reading comprehension can be understood as being composed of key 'components' or 'composite skills'. For instance, as children, first language readers are said to need five components for reading development to occur: the alphabetic principle, phonemic awareness, oral reading fluency, vocabulary and comprehension (Snow, Burns \& Griffin, 1998). Thus, due to the complexity of reading many researchers seek to explain fluent reading by analyzing the process into a set of composite skills that can be individually measured (Grabe, 1991).

Goodman (1982) viewed reading as depending on three main types of knowledge:

(1) semantic (knowledge of the world),

(2) syntactic (knowledge of the structure of the language), and

(3) graphophonic (knowledge of the sound-letter correspondence).

These types of knowledge were thought to be used simultaneously by the reader, and readers employed a series of 5 processes in reading: recognitioninitiation, prediction, confirmation, correction and termination. The Goodman model made a valuable contribution to our understanding of reading in that it firmly positioned the reader as an active participant in the reading process. While there has been strong criticism of this and similar models, and concern about their "excessive vagueness" and the questionable plausibility of some aspects of the explanation for the reading process (Stanovich, 1980, p.34), the 
reading components perspective nevertheless continues to be used and useful in research.

According to Koda (2005), Carr and Levy (1990) are credited with first proposing the component skills approach, and it has proven particularly useful for exploring individual and developmental differences and variations. The approach which entails identifying the cognitive skills underlying reading and comparing their relative contributions to performance (2005, p.19), was the subject of much research interest in the early to mid 1990s. Koda defined reading as the "product of a complex information-processing system, involving a constellation of closely related mental operations", and went on to say that "because of its multiple-skills orientation, the component approach is particularly well-suited for assessing L2 reading development" (2005, p.19). Based on an extensive review of relevant literature, Koda was also able to outline the most salient components involved in second language reading comprehension, and whilst acknowledging the influence of factors outside the reader, Koda's own work focused particularly on the cognitive comprehension processes, with the following processes being considered as core to reading:

- Decoding ability

- Vocabulary knowledge

- Text-structure knowledge

- Main idea detection

- Background knowledge

- Comprehension strategies.

However, it has become more widely accepted that it is more than just cognitive skills that contribute to reading, and this recognition has informed the construction of reading central to this thesis. Recognition of other wider influential factors within the reading equation began to be reflected in research 
in the 1990s. For instance, in 1991, Grabe listed a variety of conditions that may influence $\mathrm{L} 2$ reading. These included:

- Literacy ability in the first language

- Experience of reading for academic purposes

- Transfer of abilities from L1 reading

- Differences in the writing systems of L1 and L2

- Match of the text to the reader's level of proficiency.

These factors were then organized by Grabe into three main categories: (1) Second language acquisition and training background differences, (2) Language processing differences, and (3) Social context differences.

Based on this type of research and earlier related research, Field and Aebersold (1990) compiled a list of factors they saw as influential upon L2 reading:

- Cognitive development and cognitive style orientation at the time of beginning L2/FL study

- Language proficiency in the L1

- Metacognitive knowledge of L1 structure, grammar, and syntax

- Language proficiency in an L2/FL

- Degree of difference between L1 and an L2/FL (e.g. in writing systems, rhetorical structures, appropriate strategies)

- Cultural orientation.

In 1992, Scarcella and Oxford used the communicative competence framework devised by Canale and Swain (1980) to identify four areas of influence on reading:

1) Grammatical competence (knowledge of grammar)

2) Sociolinguistic competence (ability to use language appropriately in various social contexts) 
3) Discourse competence (knowledge of acceptable patterns in written and spoken language)

4) Strategic competence (ability to use a variety of language strategies to communicate successfully).

In the late 1990s, according to Bernhardt (2000, p.798), the following factors were considered to be central to the construct of reading in the research context:

- Affective factors

- $\quad$ Text structure

- Syntactic features

- Word knowledge

- Reading instruction (particularly in terms of vocabulary instruction for reading).

In this list, the characteristics of the text (which had become unpopular for a time, as researchers veered away from the idea of meaning residing fully within the text) have been reintroduced into the list of factors known to influence reading.

In 1998 Grabe described how this type of research generally proposed six component skills and knowledge areas:

1) Automatic recognition skills

2) Vocabulary and structural knowledge

3) Formal discourse structure knowledge

4) Content/world background knowledge

5) Skills and strategies for synthesis and evaluation

6) Metacognitive knowledge and skills monitoring. 
Grabe's own research focus was on these features and the "processing relations" perceived to exist between the various "component skills" in reading (Grabe 1998, p.58).

Exploration of the componentiality of reading has continued within the last decade, with Weir, Yang and Jin (2000) attempting to establish the parameters of the reading construct in their specific context and to investigate the divisibility of the reading construct into components. They did so to inform themselves about what measures a 'reading for academic purposes' test should contain within their own context. Their findings led them to confidently state that prior studies, their needs analysis, and the teaching and text task analyses in their study, all provided positive evidence of the separability of reading skills/strategies for both testing and teaching purposes. They concluded that theoretical literature on text processing supports a componential view of reading, as does the empirical research into the divisibility of reading (Weir, Yang \& Jin, 2000, p.118). More recently, following a similar line of reasoning, Vellutino, Tunmer, Jaccard and Chen (2007) proposed a structural model called the Convergent Skills Model of Reading Development. This defines specific reading sub-skills and reading-related cognitive abilities, hypothesizes relationships among them, and then tests these empirically. Their model incorporates no fewer than 11 such constructs, and maps intricate patterns of relationship among them.

There are, however, criticisms of the componential view of reading, and (as in many other areas of reading) strong arguments against what is often termed the 'oversimplification' of a deeply complex process. For example, there are concerns about postulating reading as a series of discrete skills that can be learned separately and that will, when assembled together, somehow comprise the ability to read. Such vagueness it appears is a persistent weakness of much 
of the theorizing of reading across the decades. Another weakness of some componential models of reading is that they fail to recognize the sometimes asymmetrical nature of the components and their relationships. Furthermore, progress in delineating some of the key components in reading and documenting their influences has also led to some researchers attributing a causative nature to the relationship between the components and the reading abilities documented.

Despite these criticisms, empirical research findings from first and second language, child and adult studies have shown that certain 'skills', 'abilities', 'factors' or 'components' are linked strongly to successful reading processes and outcomes, and that through their discriminatory qualities, they can be used to gauge reading ability and the likelihood of the reader encountering difficulty when entering a certain reading context. In some cases, for specific groups (if the factor is critical enough to reading in that genre/area and that particular context/situation) high performance in these components can be good predictors/indicators of likely future reading success.

Within a composite skills approach (a complementary understanding of reading that is also central to this study), particular skills or components can and do compensate for weaknesses in one or more of the other component areas within the reading process. This is the basis of the 'interactivecompensatory' view of reading which emerged as a focus of research in the 1980s and 1990s. The interactive-compensatory model of reading proposed by Stanovich (1980) states that not only can reading be viewed as being composed of many skills, but that these skills interact in complex ways. Moreover, Stanovich (1980) pointed out that working from this perspective allows one to see that if a reader has a shortfall in a particular process, they will be likely to rely more on other sources of knowledge, whatever 'level' 
these may come from. Prior to Stanovich's work, Masson and Sala (1978) suggested possible trade-offs between some particular high and low level processing factors, but did not recognize this as a possibly universal phenomenon. Nor did they use it to account for individual differences and developmental issues in reading ability. With this realization, the new interactive and compensatory model provided plausible explanations for empirically observed phenomena that were hitherto seemingly paradoxical.

Stanovich's compensatory hypothesis also appears to align neatly within the earlier work of Posner and Snyder (1975) who developed a theory regarding the cognitive processes involved in making lexical and semantic decisions while reading. The trend observed appeared to indicate that, with lower automaticity, there was a greater reliance on context to assist with word recognition (in line with the Compensatory Hypothesis), but that as a reader developed greater automaticity, word recognition occurred more effortlessly and 'short circuited' the action of the contextual facilitation. At the same time, the increased automaticity left many more cognitive resources available for higher level processes, and these helped the reader to integrate information and aid comprehension. This higher level processing plays a significant role in second language reading, and the compensatory hypothesis may be very useful in understanding what occurs when students in higher educational contexts are forced into patterns of overcompensating for lack of language resources, and often also a lack of relevant schema (Grabe, cited in Abisamra 2001).

More recently Yamashita (2002) investigated the relationship between second language proficiency and first language reading ability and suggested that a mutual compensatory relationship existed between the two. This also offered an explanation for why the linguistic threshold differs between readers. Yamashita acknowledged the importance and necessity of taking a "multi- 
factor approach" to reading "by operationalising conceptually complex variables", and pointed out that studies which focus on only one or two elements can only add small (but valuable) pieces of evidence to the research in this field, thus, advancing it only incrementally each time.

The incremental advancements and the changing influences of the dominant theories of the day have their strengths and drawbacks. While it is important for theorists to take into account all these factors and continually build on the 'latest' knowledge, it does make for a somewhat fragmented understanding of reading over the decades. It also makes it difficult for there to be any widespread consensus or the formation of strong theoretical foundations on which ensuing generations can build with some certainty. This is summed up well by Rapp and van den Broek:

Our understanding of the processes and factors involved in text comprehension is quite impressive, but it is quite fragmented, with a proliferation of "mini-theories" for specific components that in reality are intertwined and interact with one another. (Rapp \& van den Broek, 2005, p.276).

Although there is still much work to be done in exploring this concept, the Interactive-Compensatory model does have many implications for researchers and practitioners in reading in both the first and other languages. It is also extremely useful in informing the design of reading assessment. Of particular importance to this study is the contention of Weir, Yang and Jin (2000, p.118) that "some form of profiling of these abilities is essential" [my emphasis]. This recommendation has been incorporated into the research approach I have utilized in this study, namely the profiling of readers through thick description of a selection of salient reading-related traits, skills, abilities, approaches, attitudes, practices and beliefs. 
What became apparent when comparing these various accounts of the components, influences, and sub-skills of reading, was that the lists varied

subtly, and each was clearly linked to the specific type of reader group (usually second/foreign language) that the researchers were focusing on. For instance, it was apparent in the ways that Field and Aebersold's (1990) list varied from that of other researchers that it was tailored to the description of beginning/emergent second language readers. This made some of their categories irrelevant to readers such as those reading at an advanced academic level. A similar situation existed with some of the other lists proposed by researchers working with groups of readers who differ substantially from the group at the centre of this study.

Thus, although many views exist as to the nature, number and combination of the components, it was necessary to create a workable model specifically for the profiling of academic readers of Pacific descent in this particular study context. It was also clear that researching cognitive 'skills' alone would not be adequate to enable the holistic understanding of Pacific reading and readers. A holistic framework was needed which encompassed not only traditional cognitive and linguistic factors but also the influence of reading-related affective and attitudinal factors, together with features of readers' educational, literary and linguistic backgrounds. The framework containing the selection of factors affecting an individual's reading that I have devised and used in this study is presented in the Methodology chapter.

\section{(2.5) Understanding Reading as a Social Practice}

Bernhardt (1991, p.5) observed that "insights and data generated about reading generally fall under one of two rubrics: cognitive or social". Since there are still some inadequacies in approaching the study of reading solely from a single 'skills' perspective, this study unites the componential, interactive and 
compensatory views of reading with understandings from a socio-cultural model of reading. The theoretical frameworks that underpin the study are presented in the following chapter.

Social constructionism is the philosophical base that emphasizes that learning (and hence reading) takes place in a socio-cultural environment, and that learners (and hence readers) are active constructors of their own learning environment. All learners and readers belong to their own societies and these influence what and how they learn and thus, what and how they read (Yang \& Wilson, 2006). Reading can therefore be interpreted as a social practice occurring through 'dialogue' (either inter-mental dialogue (between people) or intra-mental dialogue (in one's own mind)), and which occurs best when scaffolding is provided in a situation of high challenge, high support.

Since each cultural context has its own purposes for using text, readers within that social context have to know how to use texts appropriately. In educational contexts, students have expectations of how texts can and should be used, based on their prior experience of texts and reading as social practice within the educational institutions which they have been part of thus far in their educational journeys. Readers entering into new educational contexts need to gain text awareness and become 'text analysts' in this new context, and will require varying degrees of support to do so appropriately (Yang \& Wilson, 2006, p.367).

Nelson (1993) documents how, until the early 1990s, reading researchers had tended to focus on the cognitive processes that happen when an individual reads, (Nelson, 1993, p.316). Nelson states that this "individualistic view of literacy" exists within a socio-cultural context which serves to foster this particular type of perspective. However, a clear movement away from this 
'solitary' view of reading has occurred in the past two decades, following the widespread recognition that cognition is firmly embedded in context. Nelson (1993, p.317) explains this well:

[n]either students nor teachers walk into class as blank slates. They bring numerous assumptions and expectations about literacy, about the teaching and learning of literacy, about education, and about the ways individuals relate to each other in the classroom. They also bring the values, attitudes, and behaviors of their home cultures and subcultures. All of these factors and more make up the social dimension of reading.

The roots of this movement did exist earlier though, and are evident in Eskey's (1976) proposal that to more fully comprehend the "total reading process" one must be willing to conceive of the reader as being simultaneously shaped by (1) their outer sociolinguistic context and culture in which "text and reader respectively could be related to a particular universe of texts and a particular society of readers", (2) their intermediate linguistic context "in which the texts could be related to the functions and forms of a given human language, and the reader to his functional knowledge of that language" and (3) their inner psycholinguistic core "where text and reader come together in the mind of a single human being". 


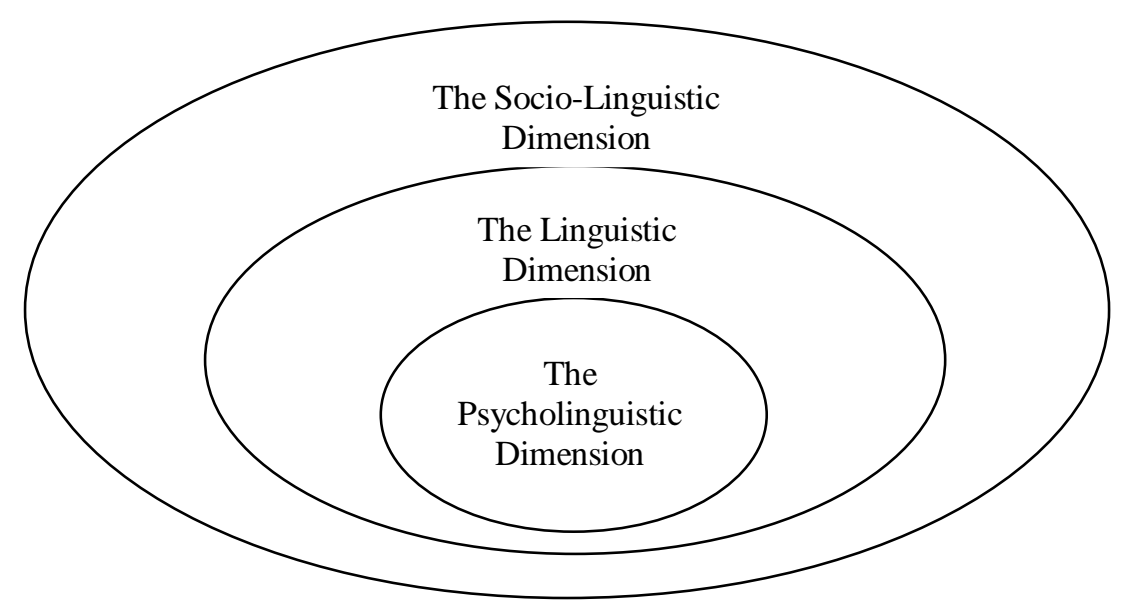

Figure 2.1: Eskey's (1976) dimensions in the "total reading process"

Eskey (1976) proposed this framework for assisting researchers and second language reading practitioners to come to a place of understanding from which more informed theories of reading could be advanced. Eskey's approach embraces an understanding of reading as being simultaneously influenced by factors from a range of internal and external sources. This study takes a similarly holistic approach to the design of a framework and methodology for investigating academic reading as both construct and process in the context of higher education, striving similarly for a framework from which to better view the "total reading process", and seeking to shed light on the dynamic interplay between the reader and their context (specifically the texts and tasks within it).

People's ways of reading are context-specific and closely linked to identity and social setting (Wallace, 2003), so there is a strong case for the influence of a reader's cultural, linguistic and educational background on academic reading. Culture, according to Behrman (2004), can be described as "an integrated pattern of behavior, practices, beliefs, and knowledge. These constitute the operating rules by which people organize themselves". 
Behrman explained that although not all members of a culture think or behave in exactly the same ways, they do all participate in it in some way, thus contributing to creating and maintaining the fundamental conventions and regulations of the culture.

When studying reading culture in an educational setting, Behrman (2004) aimed to describe the "integrated pattern of reading behavior, practices, beliefs, and knowledge, and to understand how the staff and students actively created and maintained this pattern." Consequently, Behrman's approach was to (1) study the "physical space, the shared activities, and the individual behaviors and beliefs of the participants as they related to reading", and having described these, to (2) examine how they varied and were interrelated. Following Behrman, this present study also sought to document the behaviours, beliefs and practices of real readers undertaking actual tasks with authentic texts in the context chosen for study.

In relation to the effect of cultural orientation on second language reading, Aebersold and Field (1997, p.28) commented that although

[h] ard to identify, elusive to trace, complex, and subject to individual differences, cultural orientation influences a wide variety of reading behaviors, beliefs, and performance. ... cultural orientation is important. In fact, cultural differences are pervasive and they constitute the largest category of factors that are said to influence particularly second/other language reading.

Aebersold and Field (1997, p.29-32) described the factors of second language reading that they perceived were most influenced by cultural orientation. These fall into six groups: 
1. Attitudes toward text and purpose for reading

2. Types of reading skills and strategies used in the L1

3. Types of reading skills and strategies appropriate in the L2/FL

4. Beliefs about the reading process

5. Knowledge of text types in the L1 (formal schemata)

6. Background knowledge (content schemata).

The widening view of literacy as a social practice that is always embedded in social and cultural contexts is now shared by a growing number of researchers. Hirst et al. (2004) cite Barton and Hamilton (1998), Luke (1992) and Street (2002), and state in their article on 'repositioning academic literacy' that the literacy practices of each group are contextual, situated and need to be considered in terms of "ideologies, power relations, values and identities" (2004, p.68). They explained that their view of literacy does not perceive that literacy is composed of a set of "neutral and transportable skills", nor does it advocate conceptualizing academic literacy as "a set of 'basics' that students can acquire". Instead, Hirst et al. (2004, p.68) advocated the student developing what they call "repertoires of literacy practices" and mentioned the need for students to be "apprenticed into a set of specific social practices, which will enable them to participate effectively in tertiary communities" (2004, p.69). They also strongly asserted that in the various disciplines of tertiary study, special privilege is given to certain practices and to certain voices and certain genres, and these are not always those that have been privileged in the minority student readers' home cultures. The key to participating in a community of practice is, according to Hirst et al. (2004, p.72), to adopt its practices, cultural tools, values and beliefs and ways of being so that one is then able to "contribute to its ongoing conversations". The dilemma which then arises is whether this pragmatic, yet essentially assimilationist model is the best for fostering the transition of Pacific and other 
minority culture students into university study and enabling their academic potential to be demonstrated. This debate is beyond the scope of this current thesis, but nevertheless is an important discussion which must be had at some point in the process of rethinking academic literacy in the higher education context.

It is argued that reading behaviour in literate societies is closely tied to a wide range of social, professional and personal roles (Wallace, 1986). In essence, children in literate societies are socialized into reading, and will usually want to join in reading if reading is part of the behaviour of the others in their environment. This socialization into being a reader is assumed to begin in the home, but it may not, and even if it does, it may differ from the ways that are rewarded in the education system.

Bernhardt (1991) highlighted the fact that even before a second language reader begins reading, there can be conflict. This conflict begins at the microlevel (e.g. orthography differences) and extends through aspects of the text such as grammar, and on through to the social nature of access to literacy. Bernhardt stressed that this social perspective of literacy and reading must be incorporated alongside the traditional cognitive view of reading if research into second language reading is to maintain balance and avoid being skewed. Bernhardt (1991, p.11) cites Wells' (1986) finding that

when a learner's home environment does not mesh with the school environment (generally the majority culture), the learner's attainment of literacy skills from the majority culture's point of view is retarded. The critical point here is that this retardation is not the result of cognitive deficit, but rather the result of the conflict between home and school cultures; in other words, the result of a social mismatch. 
Research seems to suggest that children with limited English proficiency in bilingual programs usually come from homes with very few books (Pucci, 1994; Neuman \& Celano, 2001) and limited literacy practices. Van Steensil (2006) investigated the relations between socio-cultural factors, the home literacy environment, and children's literacy development in the early school years and concluded that in Western societies, "where written language has a central place, no child, even when he/she is from a low income or ethnic minority family, is entirely deprived of literacy exposure at home", but that there are "considerable differences among low SES/ethnic minority children in the range of literacy activities they are engaged in" (2006, p.369). It may be that this range is key to the development of a wide variety of skills, strategies, approaches and knowledge that enable a student to approach a variety of texts in effective and efficient ways later in their educational career.

Cohen and Horowitz (2002) also emphasized the importance of the home environment as a context for "family literacy or historical-cultural practices" (2002, p.44). They concluded that teacher knowledge of the family and intergenerational literacy practices of each student's home can be instrumental in the teaching of reading. It seems likely that this also has very important implications for higher education.

According to Ackerman (cited in Nelson, 1993, p.3318), reading and writing are "learned technologies that reflect the cultural needs of a society". Scribner and Cole (1981) studied the role, functions and cognitive effects of literacy in society, and their study highlights the importance of the purposes for which literacy is learned. They point out that in the classroom the cue for reading is usually the reading/writing assignment, whereas for the Arabic speaking study participants, the primary purpose of literacy in the Vai society was to recite Qur'anic verses during religious practices. This mirrors the original purposes 
of reading in English in the Pacific, which was the promulgation of Christianity in the region. Like Scribner and Cole, Koo and Soo Hoo (2007) also touched on the important influence of the religious beliefs of parents on their children's literacy development. The significance of this religion-based reading purpose is that it encourages, privileges and rewards the development of certain sets of reading techniques which can be highly successful for the recital of religious texts but may be less suited to other contexts.

Koo and Soo Hoo's (2007) exploration of the social construction of literacy by Malaysian Chinese parents presented some telling insights into the construction of literacy that occurs within the home and school in Malaysia. The discussion applies the theoretical viewpoint of literacy as a "social discursive practice", based on the works of Gee (1996, cited in Koo \& Soo Hoo, 2007, p.73) and Wallace, 1998. According to Koo and Soo Hoo (2007, p.73),

[1]iteracy practices are the historically constituted aggregates of worldviews, ideologies, values, attitudes, behaviors and thinking of particular communities in situated contexts. Literacy practices are expressed through socially sanctioned performances and displays like acts of learning, critical thinking, listening, speaking, reading, writing and the production of texts for particular communities and contexts.

Importantly, the authors incorporated the definition of literacy provided by Baynham (1995, p.1) who emphasized that any discussion of literacy must not only include what people do, but also "what they make of what they do" and "how they construct its value and the ideologies that surround it". They also cited Barton's (1994, p.36) view that literacy involves not only the use of reading and writing within specific contexts, but also the "ways in which people bring their cultural knowledge to an activity" (Koo \& Soo Hoo, 2007, 
p.73). Thus, Koo and Soo Hoo (2007) concluded that learners' habits of reading and reading responses are the product of both their past educational and literacy experiences, and the influence of significant people and practices within their social spheres.

In the Malaysian context, Koo and Soo Hoo (2007, p.74) reported the orientation to learning as being examination-driven, valuing the "top-down transmission of knowledge and convergent thinking around standardized norms". Koo and Soo Hoo, (2007) made reference to the effects of Malaysia being a "hierarchical, collectivist society", referring to Hofstede's (1988) cultural descriptors to help understand the context of the Malaysian reader. In that context, the researchers' observations led them to note that, "children learn to take their bearings from others when it relates to opinions. The personal opinion seldom exists" (2007, p.81). They concluded that the ultimate outcome of the home, educational system and national context is a body of readers who are relatively passive, "largely uncritical and nonreflexive" (Koo \& Soo Hoo, 2007, p.82). The authors point also to the rarity of authored texts, and the students' resulting lack of exposure to texts that present an individual's views on a matter, thus resulting in readers who have been taught to avoid the "contestation of alternative views in public spheres" (Koo \& Soo Hoo, 2007, p.75). This outcome is in marked contrast with the reading qualities valued in most Western English speaking tertiary institutions, and the implications of this for a student reader from this background moving into the 'Western' model of higher education are obvious.

In studying how culture may influence language learning, Parry (1996) looked specifically at reading strategies, viewing them as a distinct group of cultural practices that relate specifically to literacy. By comparing the strategies used for completing academic reading tasks in English by students from Nigeria 
and China, Parry was able to document striking differences between the two groups. She attributed these strategy differences to language backgrounds and literacy experiences. However, Parry (1996, p.665) also cautioned that

cultural background is an important factor in the formation of individual reading strategies but that this fact should not lead to a simple cultural determinism; individual variation must always be acknowledged, and so must the fact that both individuals and cultures may change in the very process of L2 learning.

Parry acknowledged that it is important not to assume a causal relationship between culture and reading strategy choice and use, since this remains an area of considerable debate. Nevertheless, she stressed that it is very useful to acknowledge and be informed about "central tendencies within cultural groups".

Another key study which offers strong empirical evidence for the existence of distinct academic literacy profiles across national cultures and across disciplines within cultures was undertaken by Taillefer in 2005. The study is underpinned by the hypothesis that different cultures may have very different conceptions of reading for academic purposes. Taillefer cited Richardson's (1995) finding that two main broad approaches to academic reading may exist across cultures, namely (1) the comprehending approach (sometimes called the 'deep' approach) and (2) the reproducing approach (sometimes called the 'surface' approach). Taillefer explored the possible culturally-dependent sources of reading problems faced by students such as foreign exchange or international students who face the necessity of quickly becoming successful at reading in a foreign language and foreign reading literacy culture. Taillefer's findings revealed very interesting trends across the 600 students and 169 teachers surveyed in Britain, France and Spain. Taillefer concluded 
that the academic literacy culture in Britain exemplified a 'comprehending approach' in which academic reading was seen to be an integral part of study, whereas the academic reading culture demonstrated by the Spanish students more closely resembled a 'reproducing approach', and the French profile exhibited aspects of both approaches.

In a similar type of comparative study which investigated how readers complete reading tasks, Basham, Ray and Whalley (1992, p.311) found that "Latino students tended to orient themselves more toward the topic, while Asian and Alaskan students oriented themselves more toward the text and the task, though with different levels of personal authority and conviction". Overall, the authors concluded that an understanding of cultural differences in attitudes toward literacy and literate practices can make teaching staff more effective. Such studies have important implications for both teachers and students in terms of each becoming aware of possible mismatches between their respective cultures of reading. Teachers especially are encouraged to recognize that students' reading problems may stem from culturally dependent sources. Nelson (1993) argued that teachers of reading in particular need to recognize the socio-cultural dimensions of literacy and remember that students entering academic discourse communities may need to be explicitly assisted to learn the typical structures, tasks and styles that are required in that particular context.

\section{(2.6) Understanding Academic Readers through the Profiling of Readers \& Factors Affecting their Reading Success}

Currently there is little systematic profiling of readers at the higher education level. While 'profiling' is more commonly associated with psychometric and/or careers-related testing and occurs in a small proportion of educational settings as some form of general competency profiling, the research literature 
suggests that its application to reading at all levels holds great potential. Manzo, Manzo, Barnhill and Thomas (2000), for example, profile tertiary students on a scale they call the "Myself as a Reader" inventory, which enables students to self-assess their reading, study and thinking characteristics.

Factor analysis of the 148 item inventory revealed four main generic profiles to which most students could be matched. Underpinning and driving the researchers' study was the belief that "we must better understand what it means to be literate, more so than being non-illiterate, by addressing the cultural and constitutional factors that influence what people think about and do with what they read as much as how much and how well they read" (Manzo, et al. (2000, p.224).

Strucker and Davidson (2003) also set out to systematically profile adult readers in this way. Their study involved 955 adult learners and a battery of reading and language measures which were designed to describe the various 'types' of readers enrolled in adult basic education in the United States, with the overall aim, like most studies of this kind, of helping practitioners and policymakers to understand the readers and how best to respond to their specific learning needs. The learners were tested on phonological awareness, rapid naming, word recognition, oral reading, spelling, vocabulary and background knowledge, as well as being interviewed on aspects of their educational history and reading habits. Their results revealed a number of key insights into the groups measured, unique features and the configurations of features. These insights led directly to implications for the teaching and development programmes for these learners.

Another profiling-based study of adult literacy learners' difficulties in reading was undertaken by Besser, Brooks, Burton, Parisella, Spare, Stratford and Wainwright (2004). Besser et al. investigated the areas of reading difficulty 
and centered their data collection around the actual practices and experiences of learners and teachers in the classroom, observing that "there appeared to be a less than perfect match between learners' difficulties and pedagogy" (Besser et al. 2004, p.8). Specific difficulties were observed for readers in the mechanical aspects of reading including word identification, comprehension, phonological awareness, decoding and spelling. Importantly, Besser et al. document the existence of what they termed "spiky profiles", or a "scattered pattern of strengths and weaknesses".

Although studies such as these have primarily focused on the context of adult basic literacy and education, such approaches and goals have considerable potential for 'mainstream' higher education. What is common to all is the approach of identifying certain of the component factors of reading believed to be most pertinent to the group at hand, and then systematically profiling readers using those constructs. The research literature reveals the following factors commonly held to affect academic reading, and these have provided a basis for the reader profiling system designed and utilized in this study.

\section{(2.6.1) The 'Mechanics' of Reading (Specifically Reading Rate)}

The most fundamental aspect of reading involves the 'mechanics' of the reading process. Areas of interest at this level have mostly to do with cognitive language processing activities that range from phonological processing (dealing with the sounds of the language) and orthographic processing (dealing with the graphophonemic, or letter-sound, aspects), to lexical processing (dealing with words and meanings) and syntactic processing strategies (dealing with phrases and sentences) (Birch, 2007, p.6). Many of these are the focus of the profiling studies mentioned previously. 
Once these processes had been documented, it followed that research attention should shift to questioning and exploring the role of decoding efficiency in second language reading ability. According to Koda (2005, p.156) decoding efficiency is "one of the primary characteristics distinguishing strong and weak L2 learners". The key to effective decoding is efficiency, and this efficiency is developed over time with practice and is often referred to as 'automaticity'. Efficiency is important because a reader has limited working memory capacity which must be divided between the functions of "computation and temporary information storage" (Koda, 2005, p.255). From early on, researchers such as LaBerge and Samuels (1974) have suggested that when the cognitive processes of decoding during reading no longer require conscious attention (that is, they have become 'automatic' through fluency), the reader's brain has more capacity available to focus on higher order processes beginning with comprehension.

A useful indicator of decoding fluency is reading rate. Reading speed or rate is, however, still only a tentative representation of reading fluency, since the exact relationship between reading rate and comprehension remains unclear despite emphasis and research since early in the twentieth century (Meyer, Talbot \& Florencio, 1999, p.303). Nevertheless, reading speed or rate provides a rudimentary indication of reading fluency, is easily quantifiable and is thus well suited to the profiling of academic readers in this study. There are many views as to the rate/s of reading appropriate to higher education. In research into reading rate and what they term "prose retrieval", Meyer, Talbot and Florencio (1999) found that reading comprehension was affected by different reading speeds and that there appeared to be a consistent positive relationship between decreasing speed and increasing comprehension. 
After summing up numerous studies, Meyer, Talbot and Florencio (1999, p.305) concluded that while a native English speaking student of average education and intelligence would be likely to have an average reading rate of about 300 words per minute (wpm), there appears to be a general rate at which college/university students read for purposes which require "concerted long term-memory storage", and that speed is between 136-200 words per minute (wpm). Meyer, Talbot and Florencio (1999) found that the best reading comprehension performance was achieved by the university student participants at a rate of 90 words per minute (wpm). This rate is considerably slower than the rates of $300 \mathrm{wpm}$ for reading to understand, the $200 \mathrm{wpm}$ for reading to learn and the $138 \mathrm{wpm}$ for students reading to memorise which Carver's (1990) results suggested. It also differs from the $200 \mathrm{wpm}$ when reading and recalling scientific text that was found by Just and Carpenter (1980); the 144wpm and 136wpm in previous studies by Meyer and various associates (cited in Meyer, Talbot \& Florencio, 1999, p.306); and the ceiling rates of $290 \mathrm{wpm}$ and $275 \mathrm{wpm}$ above which comprehension was observed to decline (Lewandowski, Codding, Kleinmann \& Tucker, 2003).

According to Nuttall (1982, p.35), "secondary school pupils in countries where English is a second language may read at 120-150 words a minute ... University students in similar areas may read at about 200 w.p.m. but have been found to study at a rate of as little as 60 w.p.m.”. Nuttall further noted that university students from countries where there is little tradition of reading have been found to read as few as 40 words per minute. These results are similar to what I observed in my own previous research of a particular Pacific born and educated student in their first year of higher education in New Zealand, who demonstrated an average reading speed of 197 words per minute on simplified, non-academic text and an average accuracy rate of just $30 \%$ in comprehension (Davidson-Toumu'a, 2005, p.80). 
In research on Chinese ESL readers at a university in China, Weir, Yang and Jin (2000, p.4) described how a reading speed in English of 17 wpm with 70\% comprehension was the set required standard expected of Chinese students, but they found that only $1 / 3$ of graduates actually achieved this rate. Ultimately however, as Nuttall (1982, p.35) pointed out, comparisons of reading speeds in research are meaningless unless it is apparent what exactly is being compared and furthermore, a spectacular reading speed is obviously pointless if comprehension is not occurring.

Throughout the literature surveyed, it was clear that complex relationships had been documented and explored between reading speed/rate and such things as comprehension and/or recall, text type, reader purpose, and strategy use. Furthermore, the relationship is thought to be potentially two-way, with investigation being undertaken into (1) how speed or rate may contribute to certain reading effects and processes, and (2) how certain features of the text and reader may contribute to reading speed. An investigation from both these angles is necessary to shed real light on the role and nature of reading rate/ speed in academic reading. This thesis seeks to explore this two way relationship and observe how reading rate relates to other reader and text characteristics. It will be apparent thus far that the terms 'rate' and 'speed' have been used synonymously, however from this point onwards in the presentation of my study and its findings I will use the term 'rate' for reasons explained in the Methodology chapter.

Reading rate is clearly affected by many things, not least of which are the nature of the texts and readers, and the purpose for which readers were reading when measured. The importance of the purpose for which the readers were reading when their rates were measured is also discussed at length by Carver 
(1990). It is clear that each study of reading rate comprises its own unique configurations of these factors and, as in so many areas of reading research, this is what most often confounds the possibility of meaningful comparison and impedes the building of a sound base from which to draw any clear conclusions that might significantly further the field.

\section{(2.6.2) Language Proficiency (Specifically Lexical Knowledge)}

Language proficiency involves a complex range of features. Within the scope of this study it was simply not feasible to investigate all of these aspects, and so specific attention was given to the reader's lexical knowledge. The central role of vocabulary in reading comprehension has already been discussed in relation to the features of text, but vocabulary knowledge is equally important as a feature of the reader themselves which directly affects reading. The influence that the extent of the readers' vocabulary has on academic reading is most frequently documented in second language settings, since second language reading differs from first language reading in that there is not a strong language base to begin from, so linguistic (lexical) limitations are a particularly salient inhibiting factor (Carrell, 1988).

Overall, research seems to indicate that a strong and comprehensive second language vocabulary must exist before second language reading can occur, and as touched on in the previous chapter, theories about the 'threshold of linguistic proficiency' (Cummins, 1979), the 'linguistic ceiling' (Clarke, 1978, 1980, cited in Lee \& Schallert, 1997) and the 'Linguistic Threshold Hypothesis' (Devine, 1987) relate to the importance of a certain level of second language proficiency being attained before reading in the second language can really take place. Lee and Schallert (1997) investigated the relative contributions of second language proficiency and first language 
reading ability to second language reading performance. They concluded that teachers may need to tailor each learner's reading experience based more on their second language proficiency levels, and spend more time on developing a low level reader's second language proficiencies in order to bring them up to the threshold level.

In response to the many understandings of what it means to 'know' a word, the many tools that have been designed for assessment of vocabulary appear to variously test vocabulary size (how many words they know) and/or vocabulary depth (how well they know the word) (Koda, 2005, p.56). Nation and Beglar (2007, p.9) documented the relatively recent development of a Vocabulary Size Test designed to "provide a reliable, accurate and comprehensive measure of a learner's vocabulary size from the $1^{\text {st }} 1000$ to the $14^{\text {th }} 1000$ word families of English". The test is a measure that provides estimates of the test-taker's receptive vocabulary - the aspect of vocabulary knowledge most relevant to reading. In addition to the Vocabulary Size Test, several other measures have been proposed to provide insight into the vocabulary size and breadth of a reader. The other vocabulary yardstick used in this study is the Academic Vocabulary test that forms part of Schmitt, Schmitt and Clapham's (2001) Vocabulary Levels Test 5 (Version 2). Items in this test were taken from the University Word List (Xue \& Nation, 1984).

Building on the vocabulary size tests, Nation and Beglar (2007) provided a measure to estimate how close a reader is to having a vocabulary size sufficient enough to perform certain tasks. Based on $\mathrm{Hu}$ and Nation's threshold estimate of $98 \%$ coverage of a text, they believe that it is then possible to estimate the number of word families one would need to know to satisfy this $98 \%$ threshold, and thus, get an estimate of the kind of vocabulary size one would need to possess to read a given text. Their preliminary trials of 
this proposal indicated that the average undergraduate non-native speaking student who is "successfully coping with study at an English speaking university" is likely to have a vocabulary of around 5,000-6,000 word families (Nation \& Beglar, 2007, p.13).

These calculations of thresholds of vocabulary coverage may provide a rudimentary estimate of how close real readers are to "adequate unassisted comprehension" (Nation \& Beglar, 2007, p.9). However, the genres of text for which $\mathrm{Hu}$ and Nation, and Carver, have provided analysis for the vocabulary coverage needs tend to be predominantly (1) fiction (children's movies, novels, newspapers) and (2) spoken conversational English. This makes it not well-suited to this current study in which the focus is firmly on the reading of texts of an expository nature in an academic context. Key questions of relevance to this study concern the vocabulary assets of the first year readers in this research, and how these compare to the vocabulary challenge presented by the texts, and how students who wish to enter higher education can be assisted to develop large general, academic and technical vocabularies.

\section{(2.6.3) Attitudinal/Affective Factors}

In line with the growing understanding of reading as more than simply a cognitive or behavioural process, it is now beginning to be more widely accepted that readers' reading habits, their attitudes to reading, their affective responses to it, their levels of motivation, and their beliefs about both reading and themselves as readers all work to shape the readers and their interaction with text. It has been noted that as children move into adolescence "attitudes towards reading also become significantly related to reading achievement (Conlon et al., 2006)" (McHardy, 2009, p.43). It is also most likely linked to self-efficacy (the readers' opinions of their reading ability, as well as how they 
believe others view their reading), and it is commonly held that readers with good self-efficacy will be more effortful in their reading activities. The results of these effortful activities combine to influence further reading behaviour.

Readers' beliefs and attitudes evolve and develop as they progress through the levels of education. For many, as Juel (1988) repeatedly found in longitudinal studies amongst primary school children, the progress is one that becomes increasingly negative and leads to behaviours such as avoidance. It is probable that these patterns continue through consecutive educational levels unless other more positive affective experiences with text are afforded the reader. Thus, self-efficacy clearly affects performance because of its direct and indirect effects on readers' effort, persistence and willingness to persevere (Gough, 1996; Gough \& Hillinger, 1980; Hoover \& Gough, 1990; LaBerge \& Samuels, 1974; Snow, 2002, all cited in the Tertiary Education Commission (TEC), n.d.).

Readers' motivation to read in educational contexts is also a core factor affecting the success of their academic reading in higher education. In a study of the situational factors that motivate student readers to read English for Academic Purposes, Huang (2006) found that the learners studied were most willing to read when the following conditions occurred:

(1) teachers were available to answer questions

(2) key points were highlighted clearly in textbooks

(3) reading skills were taught.

Huang also found that motivation to read was influenced by whether the reader had relevant background information.

Another core reader characteristic that is known to affect reader success is interest. Reader interest is commonly studied in conjunction with factors such 
as prior knowledge (Baldwin, Peleg-Bruckner \& McClintock, 1985), and reading comprehension (Belloni \& Jongsma, 1978, who cite and build on earlier work by Bernstein, 1955; Squire, 1964; Shayner, 1968; Estes \& aughan, 1973; and Pauk, 1973). Lee (2009, p.162) recognised the theoretical importance of such affective responses as interest, and also acknowledged how they may have complex interrelationships with cognitive aspects of reading (such as prior topic knowledge and topic congruence). It has also been recognised that text features themselves also play a role in both interest and recall, as Nist and Simpson (2000, p.651) reported in their research findings on the 'considerateness' of text and its influence on readers' levels of interest.

Having extensively surveyed the research literature on the topic, Hidi (2001, p.195) reported that "interest is central in determining the ways in which we select and process certain types of information in preference to others". Hidi (2001) summed up three main lines of investigation observed in the literature on interest and reading. The first is research into the facilitative effect of interest on various reading processes, particularly comprehension and recall. The second is research establishing the variables that influence interest in reading such as text characteristics, modifications in the learning environment and an individual's own self regulation. The third is research on how, once it is elicited, interest may result in behavioural, cognitive and affective changes.

It has also been shown that relationships often exist between certain text characteristics and readers' interest. Hidi (2001, p.197) reported on particular features of text which have been found to contribute to text "interestingness and memorability". However, the ways in which these text-related factors relate to interest still requires considerable further inveatigation. Hidi's (2001) findings suggest that readers' interest may be related to a number of these factors individually. The text-related factors investigated were, however, 
clearly oriented to the semantic and meaning-related content of the text rather than the linguistic features of the text. This is logical, since the ideas in the text are most likely what one would first react to, but it does not take into account the role that the texts' linguistic and lexical features may in fact have on readers' interest responses.

Hidi (2001, p.196) concluded that research in the 1980s and 1990s demonstrated that 'both readers' well-established individual interests and their situational interests (elicited by text segments, topics, and themes) contributed to increased comprehension and learning". The studies of readers in higher education that Hidi (2001) cited indicate that:

- students who experienced situational interest while reading, demonstrated improved text recall (Schraw et al., 1995, cited in Hidi, 2001; Renninger et al., 1992, cited in Tobias, 1995)

- interest may influence the type of learning that takes place - not only the amount of information recalled but also the quality of learning and "more elaborate and deeper processing of expository texts" (Krapp,1999, Schiefele, 1996, 1998, Schiefele \& Krapp,1996, and Ryan et al., 1990, cited in Hidi, 2001; Schiefele, 1990, 1991, 1992, cited in Tobias, 1995)

Similarly, Tobias (1995) found that students in higher education for whom the text content was related to their field of interest made more accurate metacognitive judgements than students for whom the text did not coincide with their area of study interest. However, Tobias cautioned that "little is known about the cognitive processes that mediate the effect of interest on comprehension and recall of reading" (Tobias, 1995, p.399). Similarly, Alexander, Jetton, and Kulikowich (1995, p.559) pointed out that whilst a "burgeoning" number of studies have demonstrated that there is a strong relationship between knowledge, interest, and recall of text, how these factors 
are associated and what influence they collectively have on learning within academic disciplines has "remained a puzzle".

Nevertheless, Alexander, Jetton and Kulikowich have emphasized that interest is a vital factor in the reading equation, and pointed to the consistent finding that

learning in any domain cannot be understood as a purely rational, "coldly cognitive" enterprise (e.g., Pintrich, Marx, \& Boyle, 1993; Pintrich \& Schrauben, 1992; Schunk \& Meece, 1992). ... Therefore, it seems vital for future explorations of learning and development to embrace the multidimensional, interactive role of cognitive and noncognitive factors.

(Alexander, Jetton, \& Kulikowich, 1995, p.559)

Another important affective reader response to text is that of perceived difficulty. Research literature exists on readers' perceptions of text difficulty and its effects in the context of compulsory schooling levels, but relatively little exists in the context of higher education. At the primary and secondary level it has been demonstrated that perceptions of text difficulty are closely and directly linked to negative affective responses to text, which in turn led to text avoidance and the application of minimal effort to achieve and maintain pass grades. Ultimately this corresponded negatively with students' performance in reading assessments (Chapman \& Tunmer, 1995; Guthrie, Coddington \& Wigfield, 2009).

In research into the role of difficulty perceptions as an undermining motivation factor and its relation to reading achievement in elementary school students, Guthrie, Coddington and Wigfield (2009, p.338) found that for both African American and Caucasian Students, perceived difficulty was a strong predictor of reading achievement, suggesting that the relationship between negative 
affect and reading performance transcends both ethnicity and age. This kind of research evidence points fairly conclusively to the importance of student readers' affective responses and attitudinal positions and to their role in shaping students' reading experiences and determining their success.

\section{(2.6.4) The Knowledge, Use, Monitoring and Evaluation of Reading Strategies}

There are many definitions of reading strategies. Koda (2005, p.205) listed some of the definitions proposed by prominent researchers in the field:

- Weinstein and Mayer (1986) believe that the term strategy incorporates a broad array of actions that govern behaviour, emotion, motivation, communication, attention, and comprehension;

- Paris, Wasik and Turner (1991, p.692) define strategies as "actions selected deliberately to achieve particular goals";

- Rubin (1987, p.19) states that a strategy is "any set of operations, steps, plans, routines used by the learners to facilitate the obtaining, storage, retrieval and use of information";

- Anderson (1991, p.460) defined strategies as "deliberate cognitive steps that learners can take to assist in acquiring, storing and retrieving new information";

- Chamot and El-Dinary (1999, p.319) defined strategies as "mental procedures that assist learning and that occasionally can be accompanied by overt activities".

- Garner's (1987, cited in Mokhtari \& Reichard, 2002, p.250) operational definition of reading strategies is "generally deliberate, planful activities undertaken by active learners, many times to remedy perceived cognitive failure". 
Koda (2005, p.204) stated that behaviours such as monitoring reading, paying attention to cues in text to assist in organizing, understanding and retaining text, are all strategic behaviours that distinguish between skilled and lessskilled readers and "essentially characterize what is referred to as strategic reading". Most importantly for this study, there is now general agreement amongst researchers that "strategic reading" is crucial to academic contexts in which the act of reading is usually inseparable from the act of comprehending and learning difficult and unfamiliar concepts (Koda, 2005, p.204). Moreover, because findings have suggested that bilinguals who are reading in their second language use fewer strategies than monolinguals (since they are struggling with decoding and lack both the automaticity and 'headspace' (Cohen \& Horowitz, 2002)), the strategic approaches of bilinguals in higher education are of particular research importance. Nist and Holschuh (2005) summed up the situation that exists in all too many institutions of higher education:

... when students get to college, most professors expect students to think on higher levels. They are expected to apply, to synthesize, and to analyze; yet they continue to use the strategies that made them successful memorizers. ... few students receive any formal, extended instruction on how to be an efficient and effective learner. For some reason, learning how to learn has been kept a secret from them. Thus when they enter the college classroom, students may not bring with them the strategies to do anything but memorize.

There is an extensive amount of literature on a considerable number and variety of identified reading strategies, but as Block (1986) pointed out, while these studies shed light on certain reader types, the "categories of strategies vary from study to study", and many "do not adequately account for diversity within groups of readers" (Block, 1986, p.466). 
To better understand the wide array of strategies, researchers generally try to classify or group the reading strategies. Chamot and O'Malley (1994) grouped strategies into interrelated function-based clusters: Cognitive, Metacognitive, and Social and Affective. Anderson (1991, p.460) describes strategies as the "deliberate cognitive steps that learner can take to assist in acquiring, storing and retrieving new information", and proposes five strategy categories of strategies:

1) Supervising

2) Supporting

3) Paraphrasing

4) Establishing text coherence

5) Test taking.

Some reading researchers also choose to classify strategies according to time of use in the reading process, such as Paris, Wasik and Turner's (1991) strategy categories of: before reading, during reading, and after reading.

Having identified that certain lists of characteristics and strategy groupings were found to be common to more and less able readers, some researchers then sought to define lists or inventories of these strategies as a thorough and efficient means of assessing readers' strategy use. In developing one such reading strategy inventory for ESL college students, Zhang (1994) summarised a review of literature and concluded that four major sets of reading strategies existed:

1) cognitive strategies,

2) compensation strategies,

3) memory strategies,

4) test-taking strategies. 
Zhang used a rating scale of 1 to 7 to measure students' reported use of these reading strategies. The construct validity of Zhang's instrument was established and defended by virtue of having been based directly on the evidence of the systematic review of the results of decades of prior reading strategies research, and the validity and internal consistency of the items were assured statistically. The results of Zhang's study indicated that variables such as nationality, degree major and English proficiency all influenced reading strategy use in reading. It is principally by these same means - the extensive review of literature - that the academic reading strategies, habits and attitudes inventory was designed for use in this research. Zhang's 7-point response scale has also been adapted for use in this study.

Block (1986, p.472) draws the distinction between strategies aimed at (1) comprehension gathering (aiding the reader to construct an understanding of the text while reading), and (2) comprehension monitoring strategies (those that are used by the reader to monitor their own understanding and to take appropriate action when problems are encountered). In an attempt to make sense of the various categorizations, Koda (2005, p.207) compared them and found that two broad distinctions appear to underlie these many systems:

1. the distinction between cognitive and metacognitive strategies

2. the distinction between strategies that deal with local information processing, and those that deal with global information processing within a text.

The latter of these appears to concur with Block's previous distinction between meaning making and comprehension monitoring.

With the identification and classification of reading strategies, studies have then usually undertaken to investigate the nature and efficacy of reading strategy instruction interventions. Song (1998, p.42) explained that "strategy 
training comes from the assumption that success in learning mainly depends on appropriate strategy use and that unsuccessful learners can improve their learning by being trained to use effective strategies". Similarly, Cohen and Horowitz (2002) concluded that research evidence strongly suggests that readers, particularly bilingual readers, can be trained to be more strategic readers. This and other similar research suggests that reading strategy instruction (particularly comprehension strategy instruction) should receive greater attention from educators. This thesis seeks to identify patterns in strategy use and areas for development, but its scope does not extend to action research involving strategy training or intervention. This would, however, be an important line of future investigation amongst Pacific student readers.

\section{(2.6.5) Background Knowledge / Schema}

The act of reading has been described as an interactive process that occurs between the text and the reader's background knowledge, with efficient comprehension requiring an integration of the ideas in the text with one's own knowledge. According to Wallace (2003) much of the work on schema theory has been primarily derived from the work of Bartlett (1932), whose study of reconstructive memory led to a proposal that people's memory and recall abilities are influenced by their cultural experiences and world knowledge, and that they interpret information in terms of what they already know. Schema theory in effect attempts to explain how people interpret new information in relation to their existing knowledge.

Schemata are perhaps best understood as not being linguistic entities, but rather being abstract symbolic representations of knowledge which are then

expressed using language (Rumelhart \& Ortony, 1977). This is similar to Ajideh's (2003) view that "a schema (plural schemata) is a hypothetical mental structure for representing generic concepts stored in memory", and 
likens it to a "framework, or plan, or script". Wallace (1992) further expanded on the schema model in relation to reading, suggesting the existence of two main types of knowledge; (1) knowledge of the content (based on our experience of similar content in the past, and (2) knowledge of the genre (based on our knowledge and experience of similar genres in the past). Carrell (1987) also concluded that prior knowledge of both content and discourse structure influence reading comprehension.

Schema theory is essentially a theoretical metaphor for background knowledge. As such, it has been criticized for the vagueness of its theory and that it cannot be explicitly defined or measured. However, this has not been of great concern to second language researchers because it provides a very useful means for understanding how readers use background information in second and first language reading. Schema theory appears to now be relatively widely accepted in second language research and theory (Grabe, 1991), so much so that, according to Ajideh (2003, p.5), researchers commonly maintain that "the ESL teacher must provide the student with appropriate schemata s/he is lacking, and must also teach the student how to build bridges between existing knowledge and new knowledge". As with most reading theories, schema theory has implications for reading teaching and development, and particularly for second language readers. For example, schema theory in second language reading has been used as a strong rationale for certain aspects of reading strategy training, such as pre-reading activities and comprehension strategy training (Floyd \& Carrell 1987).

Since meaning "does not reside solely in words, sentences or even longer texts" and readers "bring meaning with them to texts" (Wallace 1986, p.32), this poses a very real problem for those readers from cultural or linguistic minority groups who simply do not have enough of what is considered the 
'right' type of world knowledge to bring to the text. Alptekin (2006) suggested that background knowledge is particularly important for readers for whom English is a foreign language, since so many cognitive resources are taken up in text processing that they have little left for meaning-making. Research by Cohen and Horowitz (2002) also found that cultural familiarity in texts probably acts to facilitate second language reading. However there appear to be factors that can mitigate this. For instance, Cohen and Horowitz (2002) cite Droop and Verhoeven's (1998) finding that linguistic complexity can override the benefits for readability that familiar cultural content provides.

In addition to the content related schema, it is also beneficial for readers to possess prior knowledge of (1) text and discourse structures, and (2) knowledge of genre. One factor relating to the discourse structure of expository texts that has received some recent attention is what Pretorius (2006) called 'signaled logical relations' (the text devices that explicitly signal how pieces of information in a text are related to each other). Some types of logical relations were found to be more difficult than others, and the ability to comprehend causal and adversative relations was found to be an important determinant of students' text comprehension ability. These logical relations appear to be linked to the construct of "metadiscourse" which serves to "guide readers through a text and help them to organize content as they read, thus fostering global comprehension" (Camiciottoli, 2003, p.22). Camiciottoli (2003) found that students' prior knowledge and awareness of metadiscourse is generally scarce, and that it thus warrants specific instruction. Camiciottoli also suggested that this may be even more important for students in Science and other non-Arts type subjects where students may have little awareness of "linguistic notions" (2003, p.10). 
According to Chandler (n.d.) "from the point of view of schema theory, genres are textual schemata". Importantly, an awareness of genre appears to be a factor that discriminates between more and less proficient readers. It has been noted by Wallace (1986, p.103) that "[p]oorer readers tend not to have a very clear notion of genre, largely because - like other reading-related and textrelated concepts - it develops largely through reading itself'. Livingstone (1994, p.252-3) explained that the various genres 'specify different 'contracts' to be negotiated between the text and the reader... which set up expectations on each side for the form of the communication..., its functions..., its epistemology..., and the communicative frame (e.g. the participants, the power of the viewer, the openness of the text, and the role of the reader)". Clearly, having prior knowledge of these things before reading is an advantage, as is having a clear understanding of the fact that every text has an author/s, and is connected in some way to other texts within the discipline. This understanding of the relationships between the text at hand and other relevant texts that the reader has previously encountered is referred to as intertextuality, and is linked to meaning-making and ultimately to personal transformation (Chi,1995).

\section{(2.6.6) Metacognition and Metacognitive Control}

Metacognition is usually defined as the overarching ability to think about one's thinking while performing a mental activity such as reading. Block (1992, p.320) summed up metacognition as

an ability that develops relatively late because it involves the ability to stand back and observe oneself. It is also an ability often related to effective learning and to competent performance in any area of problem solving. Experts plan, predict outcomes, and monitor their performance more consistently than do novices. 
It is generally understood that metacognition plays an important role in reading and learning, in both first and second languages, but particularly so in second language reading. Casanave (1988) explained why this is particularly so in second language reading:

for one thing, L2 readers can be expected to encounter more unfamiliar language and cultural references while reading authentic or unadapted texts than $\mathrm{L} 1$ readers would. They, therefore, may have to "repair" more gaps in their understanding than L1 readers.

Moreover, well developed metacognition appears to be a major distinguishing characteristic of so-called 'good' readers from 'poor'.

Considerable research and literature exists on metacognition, from a variety of perspectives. Brown and Campione, (1996, p.121) have provided a good summary of the activities that researchers have found the 'metacognitive skills' of reading to include:

(a) clarifying the purposes of reading (understanding the task demands, both explicit and implicit);

(b) spontaneously making use of relevant background knowledge;

(c) allocating attention so that concentration can be focused on the major content at the expense of trivia;

(d) critically evaluating content for internal consistency and compatibility with prior knowledge and common sense;

(e) monitoring ongoing activities to see if comprehension is occurring, by engaging in activities such as periodic self-review;

(f) drawing and testing inferences of many kinds including interpretations, predictions, and conclusions; 
(g) criticizing, refining, and extending newly acquired knowledge by imagining other uses of the information or counterexamples to the arguments.

In essence, the act of reading is the act of thinking, and this thinking requires both effort and skill (Brown, 1980). The study of metacognitive processes involved in reading has been very fruitful. It shows that learners may sometimes be unaware that they are not progressing well in their reading, but when they do become aware, they can use their metacognition and knowledge of strategies to remedy the situation. One of the greatest challenges for both first and second language readers, however, is "knowing when they don't know" (Devine, 1993, p.114).

\section{(2.6.7) Readers'Memory - Research on the Recall of Expository Text}

The specific study of memory and readers' memory capacities is not within the scope of this thesis, but it is important to acknowledge that it is a key factor in academic reading in higher education. Much research exists on readers' memory for expository text, for example, Meyer, Brandt and Bluth (1980), Taylor (1980) (investigating child memory), Berkowitz (1986). The majority of research on the recall of expository text pertains to its relationship with readers' understandings of text structure and the effects of this awareness or instruction on the recall of expository texts.

In the design of her research incorporating measures of memory and comprehension of text content, Block (1986, p.470) chose two methods: retelling and multiple choice questions. Straight after reading the passage, reader participants were asked to tell, as coherently and completely as possible, all that they remembered from the passage. They were not able to 
look back at the passage while doing so, and their retellings of the passage content were later scored by looking for the presence of the thesis statement and other main ideas and details that had previously been identified as such by a 'panel' of proficient readers. It is this approach that has informed the basis of the Free Recall procedure and scoring system I have used in this research.

While memory-related research investigates what the readers bring to the situation, it is important to remember that a range of factors within the reading situation may influence recall. Research on the recall of expository text has begun to reveal that certain text related factors can have an impact on the recall of expository text. For example, Lorch and Lorch (1995) found that the presence or absence of signals of text organization in the text had an effect on both recall and the strategies that adult readers in tertiary education used to encode, store and retrieve information from expository texts. It has also been suggested that the salience of passage segments is key to recall, and that this salience is related to such text characteristics as its structure and coherence, and to reader characteristics such as interest and knowledge, and to task characteristics such as the presence of inserted questions or objectives (Tobias 1995, p.400). This highlights again the need to look holistically at this and all aspects of reading-related behaviour.

\section{(2.7) The 'Good Reader' and the 'Poor reader' (and the Overarching Factor of Context)}

In reading research a distinction is frequently made between 'good readers' and 'poor readers', 'skilled' and 'less-skilled' readers, and 'proficient' and 'less proficient' readers. Using the core components of reading described in the previous section, comparative studies of these 'groups' of readers have yielded much understanding of how reading occurs and how successful reading can be promoted. The list below is a collation of relevant research- 
based findings from the past 30 years about what constitute 'good' and 'poor' readers.

$$
\begin{aligned}
& \text { Table 2.1: Selected researchers' } \\
& \text { understandings of 'Good' and 'Poor' readers } \\
& \text { in the past } 30 \text { years' research. }
\end{aligned}
$$

\begin{tabular}{|c|c|c|}
\hline Researcher/s & 'Good Readers' & 'Poor Readers' \\
\hline $\begin{array}{l}\text { (Stanovich, } \\
\text { 1980) }\end{array}$ & $\begin{array}{l}\text { Have faster word recognition } \\
\text { speeds }\end{array}$ & $\begin{array}{l}\text { May be deficient in word and } \\
\text { letter analysis skills }\end{array}$ \\
\hline $\begin{array}{l}\text { (Garner, } \\
\text { 1981) }\end{array}$ & $\begin{array}{l}\text { Focus on inter-sentential } \\
\text { consistencies }\end{array}$ & $\begin{array}{l}\text { Focus on intra-sentential } \\
\text { consistency }\end{array}$ \\
\hline $\begin{array}{l}\text { (Wallace, } \\
\text { 1986) }\end{array}$ & & $\begin{array}{l}\text { Tend not to have a clear } \\
\text { notion of genre }\end{array}$ \\
\hline $\begin{array}{l}\text { (Casanave, } \\
1988)\end{array}$ & $\begin{array}{l}\text { Prefer a top-down, meaning- } \\
\text { based approach }\end{array}$ & \\
\hline \multirow[t]{4}{*}{$\begin{array}{l}\text { (Singhal, } \\
\text { 2001) }\end{array}$} & $\begin{array}{l}\text { Keep meaning in mind while } \\
\text { reading }\end{array}$ & $\begin{array}{l}\text { Tended to lose the meaning } \\
\text { while decoding }\end{array}$ \\
\hline & Have positive self concepts & $\begin{array}{l}\text { Have a generally negative } \\
\text { self concept. }\end{array}$ \\
\hline & $\begin{array}{l}\text { Read in broad phrases and } \\
\text { skip less important parts }\end{array}$ & Read in short phrases \\
\hline & & $\begin{array}{l}\text { Ponder inconsequential } \\
\text { words and seldom skip } \\
\text { unimportant information }\end{array}$ \\
\hline \multirow[t]{6}{*}{ (Block,1992) } & $\begin{array}{l}\text { More sensitive to } \\
\text { inconsistencies in the text an } \\
\text { respond to a wider range of } \\
\text { them }\end{array}$ & $\begin{array}{l}\text { Fail to recognize or report } \\
\text { inconsistencies in text }\end{array}$ \\
\hline & $\begin{array}{l}\text { Seek to develop a coherent } \\
\text { representation of the text as a } \\
\text { whole }\end{array}$ & \\
\hline & $\begin{array}{l}\text { Use meaning-based cues for } \\
\text { evaluating if they have } \\
\text { understood }\end{array}$ & $\begin{array}{l}\text { Use or rely too heavily on } \\
\text { word-level cues and focus on } \\
\text { decoding }\end{array}$ \\
\hline & Recognition of text structure & \\
\hline & $\begin{array}{l}\text { Use general knowledge, } \\
\text { personal experience and } \\
\text { associations }\end{array}$ & \\
\hline & $\begin{array}{l}\text { Are more aware of how they } \\
\text { control their reading and more }\end{array}$ & \\
\hline
\end{tabular}




\begin{tabular}{|c|c|c|}
\hline & $\begin{array}{l}\text { able to verbalize this } \\
\text { awareness }\end{array}$ & \\
\hline \multirow[t]{3}{*}{ (Song, 1998) } & Monitor their comprehension. & \\
\hline & $\begin{array}{l}\text { Are aware of the strategies } \\
\text { they use and use them more } \\
\text { flexibly and efficiently. }\end{array}$ & \\
\hline & $\begin{array}{l}\text { Notice inconsistencies in the } \\
\text { text and do things to address } \\
\text { them. }\end{array}$ & \\
\hline \multirow{3}{*}{$\begin{array}{l}\text { (Pressley \& } \\
\text { Afflerbach, } \\
\text { 1995, cited in } \\
\text { Afflerbach, } \\
\text { 2000) }\end{array}$} & $\begin{array}{l}\text { Identify and remember } \\
\text { important information }\end{array}$ & \\
\hline & Monitor their reading & \\
\hline & Evaluate their reading & \\
\hline $\begin{array}{l}\text { (Weir, Yang } \\
\text { \& Jin, 2000) }\end{array}$ & $\begin{array}{l}\text { Know a range of strategies and } \\
\text { employ them when necessary } \\
\text { and according to task demands }\end{array}$ & $\begin{array}{l}\text { Tend to only know/use a } \\
\text { small range of strategies } \\
\text { whatever the task }\end{array}$ \\
\hline \multirow{3}{*}{$\begin{array}{l}\text { (Clarke, cited } \\
\text { in Abisamra, } \\
\text { 2001) }\end{array}$} & $\begin{array}{l}\text { Concentrate on passage-level } \\
\text { semantic clues. }\end{array}$ & \\
\hline & $\begin{array}{l}\text { Formulate and test hypotheses } \\
\text { about text before reading. }\end{array}$ & \\
\hline & $\begin{array}{l}\text { Develop a tolerance for } \\
\text { inexactness, willingness to } \\
\text { take chances and make } \\
\text { mistakes. }\end{array}$ & \\
\hline \multirow{4}{*}{$\begin{array}{l}\text { (Martino and } \\
\text { Hoffman, } \\
\text { 2002) } \\
\text { (African } \\
\text { American } \\
\text { freshmen at } \\
\text { private US } \\
\text { university), }\end{array}$} & Knew more vocabulary & \\
\hline & $\begin{array}{l}\text { Had better phonological } \\
\text { awareness }\end{array}$ & $\begin{array}{l}\text { Had poor phonological } \\
\text { working memory. }\end{array}$ \\
\hline & & $\begin{array}{l}\text { Working memory capacity } \\
\text { was not as great as the higher } \\
\text { ability students }\end{array}$ \\
\hline & & $\begin{array}{l}\text { Needed strategies to address } \\
\text { decoding and similar lower } \\
\text { order reading skills }\end{array}$ \\
\hline \multirow{2}{*}{$\begin{array}{l}\text { (Bouvet, } \\
\text { 2002) } \\
\text { (reading in } \\
\text { foreign } \\
\text { language: } \\
\text { Melbourne } \\
\text { university } \\
\text { students }\end{array}$} & $\begin{array}{l}\text { Better second language } \\
\text { knowledge helped them use } \\
\text { more effective and } \\
\text { sophisticated strategies. e.g. } \\
\text { Favouring energy efficient } \\
\text { moves like skipping. }\end{array}$ & $\begin{array}{l}\text { Had to resort to facilitative } \\
\text { processes to help overcome } \\
\text { textual unfamiliarities } \\
\text { mechanically. }\end{array}$ \\
\hline & $\begin{array}{l}\text { Used 'macroframe } \\
\text { elaboration' - formulated }\end{array}$ & $\begin{array}{l}\text { Used macroframe } \\
\text { elaboration but not as much }\end{array}$ \\
\hline
\end{tabular}




\begin{tabular}{|c|c|c|}
\hline \multirow[t]{3}{*}{$\begin{array}{l}\text { studying } \\
\text { French) }\end{array}$} & $\begin{array}{l}\text { 'snapshots' of understanding } \\
\text { periodically through the text } \\
\text { and as they progressed, they } \\
\text { put the pieces together to get a } \\
\text { global picture of the text. }\end{array}$ & or as systematically. \\
\hline & $\begin{array}{l}\text { Relied very little on the use of } \\
\text { external sources }\end{array}$ & Relied on external sources. \\
\hline & $\begin{array}{l}\text { Read in such a way as to avoid } \\
\text { breaking the reading flow }\end{array}$ & \\
\hline \multirow{14}{*}{$\begin{array}{l}\text { (Duke \& } \\
\text { Pearson, } \\
\text { 2002) }\end{array}$} & Were active & \\
\hline & Had clear goals & \\
\hline & Constantly evaluated & \\
\hline & Previewed text before reading & \\
\hline & Made predictions & \\
\hline & Read selectively & \\
\hline & $\begin{array}{l}\text { Constructed, revised, } \\
\text { questioned meaning }\end{array}$ & \\
\hline & $\begin{array}{l}\text { Dealt with unfamiliar words, } \\
\text { concepts and gaps in } \\
\text { understanding }\end{array}$ & \\
\hline & Integrated prior knowledge & \\
\hline & Thought about authors & \\
\hline & Monitored understanding & \\
\hline & Evaluated text quality & \\
\hline & Read different texts differently & \\
\hline & $\begin{array}{l}\text { Found reading satisfying and } \\
\text { productive }\end{array}$ & \\
\hline \multirow{3}{*}{$\begin{array}{l}\text { (Lau \& } \\
\text { Chan, 2003) } \\
\text { (L1 Chinese } \\
\text { secondary } \\
\text { school } \\
\text { readers } \\
\text { reading } \\
\text { Chinese } \\
\text { texts) }\end{array}$} & & $\begin{array}{l}\text { Less capable in their use of } \\
\text { cognitive and metacognitive } \\
\text { reading strategies to } \\
\text { comprehend Chinese text }\end{array}$ \\
\hline & & $\begin{array}{l}\text { Could only use simple } \\
\text { strategies and that they may } \\
\text { also face deficiencies in their } \\
\text { higher order thinking skills } \\
\text { as well as sophisticated } \\
\text { reading skills }\end{array}$ \\
\hline & & $\begin{array}{l}\text { Showed significantly less } \\
\text { intrinsic motivation than } \\
\text { good readers }\end{array}$ \\
\hline $\begin{array}{l}\text { (Cziko, 1980; } \\
\text { Horiba, }\end{array}$ & & $\begin{array}{l}\text { Were more heavily involved } \\
\text { in word-level than in }\end{array}$ \\
\hline
\end{tabular}




\begin{tabular}{|l|l|l|}
\hline $\begin{array}{l}\text { 1990, cited } \\
\text { in Koda, }\end{array}$ 2005, p.39) & & discourse-level processing \\
\cline { 2 - 3 } & & $\begin{array}{l}\text { Had "seriously restricted } \\
\text { word recognition skills" } \\
\text { spend considerable time and } \\
\text { energy in their visual } \\
\text { sampling, which constrains } \\
\text { their ability to use multiple } \\
\text { information sources. }\end{array}$ \\
\hline $\begin{array}{l}\text { (Lau, 2006) } \\
\text { (secondary } \\
\text { school aged } \\
\text { students in } \\
\text { Hong Kong) }\end{array}$ & $\begin{array}{l}\text { Had a more comprehensive } \\
\text { metacognitive conception of } \\
\text { their own reading abilities than } \\
\text { poor readers. }\end{array}$ & Had better motivation \\
\hline $\begin{array}{l}\text { (Pretorius, } \\
\text { 2006) }\end{array}$ & $\begin{array}{l}\text { Searched for relationships } \\
\text { between masses of details and } \\
\text { find chunks of info to join } \\
\text { together to create } \\
\text { representation of text as whole }\end{array}$ & $\begin{array}{l}\text { Found it challenging to } \\
\text { understand logical } \\
\text { connectives in text }\end{array}$ \\
\hline
\end{tabular}

Some of these characteristics particularly stand out as being most widely considered as indicative of reading skill. The importance of metacognitive control is clear in the literature, and is supported by Block (1992, p.322), who reported that "there is some tentative evidence suggesting that metacognitive control, a globally oriented process, distinguishes more and less skilled L2 readers". Horiba's study (2000, p.253) offered strong evidence that good readers are those with flexible control over the allocation of their cognitive resources to a variety of types of text processing, whereas poor readers, who already had less control over their processing, became even less flexible in their processing as they encountered more linguistically and conceptually demanding texts.

Lau and Chan's (2003) findings also suggest that there is a strong link between reading comprehension ability and strategy use and reader motivation (particularly intrinsic). The consistency of these findings with both native 
speaking and second language readers seems to indicate that what occurs at the higher levels of processing during reading appear to be universal cognitive activities, independent of the language system. For this reason, both cognitive and motivational factors should be considered by all educators attempting to plan programmes to assist less skilled readers.

Similarly, Song (1998, p.49) states that "many L1 and L2 reading researchers have demonstrated that strategy use and awareness of reading strategies are different in more or less proficient readers, and that more proficient readers use various types of strategies, and they use them in more efficient ways". The finding that good readers use more reading strategies more effectively is also supported by Weir, Yang and Jin (2000, p.118). They concluded that most of the medium to high ability students appeared to use a range of strategies, whereas the weakest students "appeared to have only one style, of slow careful reading" for whatever task was presented to them.

Bouvet's (2002) findings, on the other hand, indicated that the proficient and less proficient readers appear to use similar strategies, but make use of them in different ways and to different extents. Bouvet $(2002$, p.1) concluded that the main difference between the more and less proficient readers "resides in the ability some readers have to integrate meaning and construct text in a cohesive and synthetic fashion".

Interestingly, Lau (2006) found that the differences between the 'good' and 'poor' readers emerged more when dealing with expository rather than narrative text. This has significance for academic reading and literacy development issues in higher education wherein expository texts form the foundation of learning. However, in a discussion of 'good' and 'poor' readers it is also important to recognize that the results of some studies on the 
importance of second language proficiency have shown that limited control of the second language may cause a normally good reader to revert to poor reading behaviours.

Thus, a person may in fact know, and know how to use and monitor, strategies in their first language, but when reading in the second language these are not able to be utilized for a number of complex reasons. Personal experience also suggests that there may be varying degrees of overlap between the sets of reading strategies, skills, habits, attitudes and behaviours that are rewarded in the two language contexts. Readers may find that they were 'good readers' in their previous educational context, only to struggle and be labeled "poor readers' in their new context because that particular context assumes and rewards a particular set of characteristics that the reader has had neither the need nor the opportunity to acquire. Overall then, the view expressed by Clarke (1980, p.206) is probably the most balanced: "it may be inaccurate to speak of "good readers" and "poor readers", but of good and poor reading behaviors which characterize most readers at different times".

Another difficulty with classifying a set of characteristics as being definitive descriptions of 'good' readers or 'poor' readers, and about giving learners fixed labels, is that in every situation there are the ever-present, all-pervasive and overarching factors of context and purpose. The question which necessarily arises is whether the 'good' reader in one context will also be a 'good reader' in another when they transfer that present set of skills, habits, beliefs, attitudes and approaches to another context? As this study is situated within the first year of higher education the next question which arises is about what it is that particularly defines a 'good reader' in the context of higher education, and whether it is again a fairly constant and definable set of 
competencies or whether it varies from discipline to discipline, being influenced by context?

Nevertheless, it is clear that researchers still refer to 'good' and 'poor', or 'proficient' and 'less proficient' readers, and constantly seek features whose measurement may yield the desired discriminatory ability and be helpful for identifying students with potential weaknesses. The lists which emerged from the survey of literature on reader characteristics and abilities found commonly in skilled readers and less skilled readers are also a valuable starting point for the profiling of readers. They are therefore used in this study as the basis for the design of the reading strategies, habits and attitudes inventory, which is described in the Methodology section.

\section{(2.8) Linguistic/Ethnic Minority Background Students \& Readiness for Academic Reading in Higher Education}

Given the findings that home culture influences aspects of academic reading, it follows that students of linguistic or cultural minority descent in higher education are likely to bring with them reading skills, beliefs, behaviours and knowledge that have their own particular characteristics. One common approach to understanding the experiences of English second language readers and readers of ethnic and linguistic minority descent is to investigate whether differences exist between them and native English speaking readers, and what the nature of these differences are. What follows is a consideration of relevant studies on linguistic/ethnic minority students in higher education, and research literature on the phenomenon of 'readiness' for tertiary study.

Lonon Blanton (1993) wrote from the perspective of having taught at an urban university with a high refugee student population. Similarly to my own previous research (Davidson-Toumu'a, 2005), Lonon Blanton (1993, p.235) found that the refugee student participants approached text passively, as if they 
had no role to play in the reading process. Although they valued education, they had seemingly little understanding of what it would take to achieve in education. Lonon Blanton also found that if a student reads a text but finds no way to connect it to themselves, they have understood it only in a very superficial way, having decoded it but not truly comprehended it. In addition to this, the students studied were observed to rely on the text so heavily for its meaning that when the text was taken away and they were asked questions, they were rarely able to answer questions or discuss the topic. This left them feeling extremely inadequate.

In what Lonon Blanton (1993, p.237) termed "the jargon of our trade", these students would commonly be referred to as "not having been "socialized" into the "academic discourse community". In essence, they had not developed the persona of a reader. The solution appeared to be to facilitate and support the students as they developed an awareness of themselves as readers. Lonon Blanton saw that a reader-based pedagogy, not a text-based one, is important for teaching these students about creating connections between themselves and the text, and encouraging them to think about themselves as readers.

In a study involving only African American students, Martino and Hoffman's (2002) data showed that this group still exhibited problems with the more mechanical aspects of reading such as decoding and phonological working memory. The researchers thought that this may indicate that the mechanical aspects which are largely assumed to be firmly developed by the tertiary level, are in fact not fully developed for many students from this (and possibly other) groups. This raises the issue that it may be of little value to focus on the teaching of more advanced reading strategies until one has established whether the problem in fact lies at a more fundamental level of the reading process. 
The phenomenon of minority students not being 'prepared' for higher education reading demands has also been documented closer to home in Australia. Sugirin (1999), and Hirst, Henderson, Allan, Bode and Kocatepe (2004) among others, have highlighted the changing composition of the undergraduate cohort and the fact that long-held assumptions about the skillbase students bring with them to the first year of university can no longer be maintained. As Hirst et al. pointed out, "there is no guarantee that students have been equipped by their previous life experiences to cope with the academic or tertiary literacies required of them". Due to the rapidly growing popularity of Australasian universities internationally, challenges have arisen around the concern that "international students from non-English speaking backgrounds may not have the necessary skills required to participate in the mainstream" (Sugirin, 1999, p.1). Importantly, Sugirin argued that while the challenges and importance of academic reading are high, research into the comprehension processes of this group of students is comparatively scarce.

Existing research has tended to use 'think-alouds' as the major research method, but Sugirin (1999) argued the value of complementing such a protocol with other methods, especially a multiple-choice test with in-depth interview to be carried out immediately after the think-aloud procedure. Such a combination allowed Sugirin to confidently document procedural aspects of students' reading comprehension. The relevance of Sugirin's work for this thesis lies in the use of authentic and discipline-specific texts for the procedures and the fact that, having profiled the readers, practical implications were then able to be offered for the teaching of these students. Sugirin is clear that "all lecturers should share responsibility of helping the NESB students to develop themselves into proficient readers in order to be able to participate in the mainstream". The complexity of the task of academic reading for NESB (Non-English Speaking Backgrounds) students is stressed, as is the fact that 
students approach tasks in a number of different ways, not all of which are successful (1999, p.13). Sugirin's argument that the utilization of multimethods in reading research has the potential to result in superior and more comprehensive insights into the reading comprehension processes employed by ethnic and linguistic minority student readers, providing strong support for the holistic approach chosen for use in this research.

A similar study of minority students' reading readiness for higher education is found in Bell's (2007) research into the reading practices of postgraduate Thai students at an Australian university. Bell investigated how and to what extent students' home country and Australian experiences shaped their reading practices while studying in Australia, and found that "during the first semester the six Thai participants displayed an awareness that their home country reading experiences and background knowledge had not fully prepared them for their graduate and postgraduate studies in the Australian environment". Bell went on to describe how these students "soon became aware, moreover, that many of the strategies they brought with them from Thailand were not effective for the amount and type of reading they had to tackle" (Bell, 2007, p.56). Thus, a certain degree of mismatch was recently observed in Australian universities, and I argue that this is most likely to be the case in New Zealand too.

Such acknowledgments of the potential differences that some readers may bring to higher education usually leads to discussions of 'readiness' for higher education. The particular issue of reading readiness amongst ethnic and linguistic minority students in higher education is one which requires urgent attention and research in the New Zealand higher education context. 
This field of study is already well established in the United States where the American College Testing (ACT) system and a series of prior tests in the secondary and upper primary system exist to ensure that the situation of 'underprepared' readers from various ethnic minority groups, such as African American, Hispanic and Native Americans, is relatively well documented. It appears to be widely recognised in the US that many students entering college (university) are 'underprepared' for the demands of reading in higher education. For example, in a report by the ACT states that in 2005, approximately half of all high school graduates would be ready to meet the demands of college level reading (ACT, 2006, p.1). Statistically, a significant proportion of this percentage has always been made up of ethnic and linguistic minority populations. The literature surveyed indicates that concern about these same issues has been rising for some time across the English speaking world. Concern is also expressed that this problem is not new, and has apparently not been responded to by those who have the power to begin to address it.

A more comprehensive investigation of university readiness ('college readiness' in US literature) was undertaken by Conley (2008), who noted that a deficiency in previous considerations of general college readiness was that they failed to cover a wide enough range of the factors. Conley (2008) sought to better reflect the range of facets involved, proposing a four-part model of readiness factors:

1) key cognitive strategies (for example, analysis, interpretation, precision and accuracy, problem solving and reasoning)

2) key content knowledge (major theories and content areas central to the discipline)

3) academic behaviours (such as academic self management behaviours) 
4) contextual skills and knowledge (e.g. contextual knowledge associated with college application, entrance and acculturation).

Conley observed that the development of the cognitive and metacognitive capabilities of incoming students is "at the heart of college readiness" and that a more comprehensive model of college readiness is needed to assist in drawing attention to the difference between the concepts of being "college eligible" and being "college ready". However, what the four-part model notably lacks is reference to the centrally important aspects of language proficiency and/or literacy ability of learners. Nevertheless, the framework for conceptualizing the overall readiness of students for college/university is useful in that it allows the situation of reading skills in relation to the other key facets of readiness. Compounding the issue of under-preparedness is that it has also been shown to be both self-perpetuating and self-exacerbating.

Dreyer and Nel's (2003, p.350) African studies demonstrated that when underprepared students were pressed to read, they most often selected ineffective and inefficient strategies and approaches to the tasks. Consequently, their attempts became even less fruitful and even more frustrating. This was though to be due to limitations in both knowledge of university-appropriate reading strategies and metacognitive skills. It is however noticeable that in the majority of these studies, the orientation is clearly towards establishing apparent deficiencies in the minority readers, with little acknowledgement of the responsibility of colleges and universities for ensuring their own 'readiness' to provide appropriate, relevant and empowering educational and literacy experiences for these learners.

The prevailing understanding of students not being ready for the demands of tertiary academic reading is often further complicated by the unspoken beliefs 
held by staff within higher education. Grimes and David (1999, p.2) cite London's (1980) research which documented faculty beliefs that "students are responsible for their own success or failure", and that any areas of weakness that are in need of development are automatically attributed to "deficiencies" in the student's "character, mind, or social background". London's findings also suggest that faculty may even express ambivalence toward the mission of helping 'at-risk' students for these very reasons. Although a little dated, Obah's experience in the African context is revealing. Obah (1982) documented tertiary staff members' often incredulous responses to her attempts to raise awareness of the importance of academic reading in higher education and the need for it to be taught in some contexts.

\begin{abstract}
"Teaching what?. . Reading?. . To infants?. . . adults?... on this campus?... I don't understand. . what is there to teach?" The tone of stunned disbelief shows, first of all, that most people think that reading as a subject in the curriculum belongs in primary schools, where children learn to read. They may allow that reading belongs to some extent in secondary schools where students, if they wish to pass the secondary school leaving examination or the university admission screening examination, must practice comprehension and summary exercises. The tone of disbelief, secondly, shows that most people know only the two skills that are taught everywhere: "Reading to break the code" and "Reading to get by." That there is anything beyond these different techniques of reading, different purposes for reading, different levels of reading and comprehension, different rates of reading, all of which we go on acquiring and perfecting through life - is unknown or known only in a vague, incidental way.
\end{abstract}

(Obah, 1982, p.316)

In cases where the reality of reader under-preparedness is brought to educators' explicit attention, the questions which usually arise are why the 
phenomenon occurs and what should be done about it (if indeed it is the place of higher education to do anything)?

Many would suggest that under-preparedness occurs because schools and universities are operating at different levels and often at cross purposes when it comes to literacy, particularly in relation to the subject of English. Green (2006), drawing upon the work of Knights (2004), documented "a growing perception that dichotomous paradigms of English (or rather Englishes) now exist in schools or colleges and university". Green (2006, p.276) also stressed that
attached to each paradigm are associated pedagogies and practices which enshrine (often tacitly) their own particular codes and agendas, and imply a relationship both between teachers and the material taught and between teachers and learners.

To establish the true foundations for teaching and learning, Green concluded that there must be a balance between "delivery' and 'inquiry", and that this "depends upon scrutiny of the respective roles of lecturers and students, the functions of pedagogical content knowledge (Turvey, 2005) and evaluation of learner readiness (Daly, 2004)" (Green, 2006, p.276).

In their research into the student experience in Australian universities, McInnis and James (1995) pointed out that a significant divide exists not only between school or college and university, but also between students and academics. This recognition is fundamental in beginning to define and refine pedagogic practices. Unless thinking about teaching begins with thinking about learners and their readiness to learn, a "short circuit" inevitably occurs in the process of adapting to university study. To ensure the appropriate support, challenge and development for students occurs, objectives for teaching and learning need to 
engage as much with the students' actual abilities as they do with lecturers' perceptions of what these should be. This is not to imply that subject content needs to change or that the students should be patronized by being offered over-simplified courses. At issue is the need to develop an understanding of where students come from in terms of their learning, and the pedagogical processes and rationales they have experienced prior to entering university study, so that teacherly practice facilitates their progression rather than reinforces difficulties (Green, 2006, p.277).

Part of acknowledging where student readers come from is also to understand what academic reading expectations have been required of them prior to entry into University. The prior educational environments of students and the particular sets of skills, strategies, approaches and reading behaviours which they privilege and reward, shape student readers. Building an understanding of students' reading histories enables a deeper understanding of both the reader themselves and the education system within which they have journeyed.

Along these lines and in the interests of the issues surrounding the transition of students into tertiary from upper secondary levels, it is worthwhile noting the stated academic reading goals set for readers in the years of education just prior to entering university both in New Zealand, and across the wider OECD countries. The New Zealand Ministry of Education sets out the literacy requirements for achieving 'University Entrance'. At the time of this study's literature review the 2004 requirements were available on the New Zealand Ministry of Education website. English standards fulfilling the literacy requirement for university entrance from 2004 included:

- $\quad$ responding critically to written text(s) studied 
- completing independent research on a language or literature topic and presenting conclusions in writing

- $\quad$ analysing extended written text(s)

- $\quad$ analysing short written texts

- $\quad$ reading unfamiliar texts and analyzing the ideas and language features

- $\quad$ investigating a language or literature topic and presenting information in written form

- investigate a theme across a range of selected texts and evaluating the outcomes of the investigation

- investigating a theme across an inclusive range of selected texts

- $\quad$ reading closely and evaluating the effectiveness of transactional written texts

- $\quad$ reading an inclusive variety of written texts and recording the reading experience

- $\quad$ reading transactional written text closely

In essence, it could be argued that this list reveals the core skills which are thought to be essential for 'university entrance' at the upper secondary school levels in New Zealand, and that when summarised, they relate to:

- reading to investigate a topic/theme across a range of selected texts (that is, research)

- reading to respond critically to text

- reading to analyse ideas and stylistic features of text

- reading to evaluate the effectiveness of text

An in-depth investigation the degree of overlap between these and the core reading processes of the first year of university is beyond the current scope of 
this thesis, but is an issue which does warrant closer attention in research efforts toward issues of transition and university preparedness.

Another measure of reading in upper secondary levels of education can be found in the PISA 2000 measure which was used to compare New Zealand and other OECD countries on literacy levels in 2000. The reading literacy measure was divided into Proficiency Levels on the three reading sub-skills: (1) information retrieval, (2) text interpretation and (3) evaluation. The following descriptors are from an excerpt of the PISA 2000 Proficiency Levels Measure (Level 5), and provide an indication of what was expected to be demonstrated by the top percentage of all secondary school readers:

- Retrieving information (locating one or more pieces of information in a text)

Critically evaluate or hypothesise, drawing on specialised knowledge. Deal with concepts that are contrary to expectations and draw on a deep understanding of long or complex tests.

- Interpreting texts (constructing meaning and drawing inferences from one or more parts of a text)

Either construe the meaning of nuanced language or demonstrate a full and detailed understanding of a text.

- Reflecting and evaluation (relating a text to one's experience, knowledge and ideas)

Locate and possibly sequence or combine multiple pieces of deeply embedded information, some of which may be outside the main body of the text. Infer which information in the text is relevant to the task. Deal with highly plausible and/or extensive competing information.

(Comparative Education Research Unit, Ministry of Education, 2004, p.7)

These descriptors embody what internationally is considered to be achievable by the most capable readers at this age level. They thus provide a basis of comparison for OECD countries (with which New Zealand seeks to align 
itself) and can therefore be thought of as fairly representative of the kinds of abilities students may be expected to demonstrate in their first year of higher education. Yet, it was estimated that less than $6 \%$ of the roughly three and a half thousand Pacific descent student readers studied in 2000 in New Zealand were able to perform to this level at the age of 15 or 16 years (Ministry of Education, 2004).

In upper levels of compulsory education, Brown and Campione point out that

[m] uch of what is called reading ... is indistinguishable from critical thinking and studying. Students are not only required to decode, they are also required to understand the meaning, critically evaluate the message, remember the content, and apply the newfound knowledge flexibly and creatively.

(Brown \& Campione, 1996, p.121)

Similarly, Pretorius (2000, p.6) states that in higher education "[n]ot only do students have to read, understand and critically evaluate numerous texts, they also have to accumulate systematically conflicting information from various sources and integrate it into coherent knowledge systems or schemas on various topics".

At the tertiary study level though, only a few studies exist that have specifically and clearly set out to document the academic reading tasks of higher education. In the assessment schedules of the Media Studies course analysed by Railton and Watson (2005, p.188), the three main processes required of students were to:

- comprehend key concepts

- apply key concepts in another context

- $\quad$ use and critique a range of key concepts 
Thus while some international studies exist which seek to more explicitly document the nature of the academic reading literacy in upper compulsory and tertiary study, there is much more which needs to be understood about New Zealand's academic literacy situation and particularly the transition experiences of students of Pacific descent.

Despite the often somewhat controversial nature of the debate about the reasons for differing levels of preparedness amongst students entering higher education, the study of readiness for tertiary study is valuable because of the implications it has and the questions it necessarily raises for teaching and learning situations in higher education. For instance, such studies often point to the necessity of an acknowledgement of responsibility on the part of educational institutions. Grimes and David (1999, p.13) concluded that "[a] commitment to universal access means that open-door colleges cannot ask if the students are "college material"; they are tasked with ensuring that the colleges are "student material" (Gleazer, 1970)". Increasing participation carries with it conditions of success and progression, and this is of utmost importance to minority students in higher education because, as Tinto (2004) noted, "access without support is not opportunity". Due to the importance of this issue, and the key role of the first year of higher education study in shaping the likelihood of a student staying on at university, the first year is a crucial place to examine reading and study readiness.

In terms of the 'diagnosis' of readiness, Berhman and Street (2005) promoted the use of content-specific rather than content-general reading assessments for university entrants. They argued that

content-general reading placement tests that measure comprehension across a variety of subject areas may have limited utility in academic placement decisions. As an 
alternative to using content-general reading placement tests, it is suggested that reading placement tests be specific to courses for which placement decisions will be made.

(Berhman \& Street, 2005, p.17)

At the heart of all of this is the premise that students' difficulties in managing the transition into higher education and its literacy practices are not necessarily a reflection of their academic impoverishment, but rather are a reflection of (1) the profound changes in the learning environment they experience, and (2) their unfamiliarity with the lecturers' expectations (Green, 2006, p.281). It is therefore important that consideration is given not only to the content-base of the subject for study, but also to the pedagogical and practical dimensions of subject knowledge. In doing so, it is important that a clear path is steered away from a deficit mentality that positions learners, especially those from non-traditional backgrounds, as being 'lacking' or 'deficient'.

\section{(2.9) Pacific Student Readers in New Zealand}

Some researchers have argued that the apparent under-preparedness of readers from non-traditional backgrounds is a consequence of larger cultural and societal practices. Parry (1996), for example, was concerned that the "significance" of written language varies a great deal from one cultural context to another, and that this is influenced by many factors, including how the written language is related to the everyday spoken language of the people, the social functions the written texts are expected to serve in that community, and the way in which the knowledge of the writing system is transmitted from one generation to the next. In relating this to Pacific cultures and societies, both within NZ and Oceania, the situation was summarised well by Mangubhai 1995, p.14): 
When we look at the type of literate behaviours seen in Pacific societies, especially in the Eastern Pacific, we note that reading of the Bible and other religious materials has become a part of the culture of the people (Clammer 1976, Spolsky et al. 1983, Mangubhai 1987, Crowley 1989). ... Activities in Pacific societies tend to be communal and not solitary and literacy practices like reading for one's own pleasure or exploring information or knowledge for the sake of interest do not fit well into this pattern. The practice of reading widely is not encouraged either by the way many rural homes are built ... Living conditions in conjunction with cultural practices have not encouraged the development of the practice of wider reading.

Both reading and writing have, since their introduction, traditionally served a limited purpose within Pacific societies, but from this it should not be inferred that reading and writing are not regarded as important by parents for their children. In fact Pacific children are usually told repeatedly both by their parents and their teachers about the importance of reading and writing in the school contexts. However, according to Mangubhai (1995, p.15), the rhetoric is not matched by corresponding encouragement or social approval for schoolbased activities to spill over into the society.

Research specifically into the academic literacy of Pacific students in postcompulsory levels is scare. In such studies reading may play a small part, but is usually parceled together with writing and labeled 'literacy' issues. Others such as Fanene's (2006) study looked specifically at the academic writing of students of Pacific Island descent. Apart from my own Masters research (Davidson-Toumu'a, 2005), Masters degree research carried out by McHardy $(2009$, p.6) on the characteristics of adult readers in entry level tertiary settings in New Zealand is the nearest study to the issue of academic reading and Pacific students in higher education - although in McHardy's investigation of 
the reading related characteristics of $52 \mathrm{NZ}$ adults, only two participants were from Pacific heritages. These students were enrolled in Private Training Establishments and engaged in employment skills courses, security work training or trade skills courses, and her study reports that

results from IALS (1996) showed $75 \%$ of Pasifika adults failed to meet the minimum levels of literacy competence considered necessary for the knowledge society (Gibbs,2008). Ten years on from IALS (1996), results from ALL (2006) showed Pasifika continue to be over-represented in the low levels in all sections of the survey (Satherley \& Lawes, 2008b).

McHardy investigated the decoding skills, word reading skills, sentence comprehension skills and receptive vocabulary knowledge of the participants, and also looked at their self-belief in reading ability and the value they placed on reading, as well as their reading habits. While some correlations were found, overall McHardy found no apparent relationship between the actual reading skill of the readers and their perception of that skill, or any link between the value they placed on reading and their actual skill. However it was noted that generally the skilled readers in this study read a broader range of materials and read more regularly than less-skilled readers in this study. While only a small proportion of McHardy's participants were of Pacific descent, this study does indicate just how complex the issues surrounding reading are, and how difficult it is to simply predict the reading ability of adults in tertiary study.

On the whole, current studies on Pacific descent students in higher education appear to be primarily concerned with issues of retention and of general student experiences. The scarcity of such research on the academic reading literacy experiences and abilities of Pacific students at the tertiary level means that I have found it necessary to read relevant research at lower educational 
levels, and comparable bodies of minority students reading in overseas universities in order to inform me of the likely issues at stake. The most relevant national study of secondary school literacy amongst Pacific students is the work done on the academic literacy and reading abilities of Pacific students by the Programme for International Student Assessment (PISA). PISA carried out a widespread study on the reading literacy, mathematical and scientific literacy abilities of 15 year olds in 28 OECD member countries, aiming to achieve internationally comparable measures of educational achievement. From this data, reading related information which is relevant to the educational achievement of Pacific students has been collated by the Ministry of Education in New Zealand.

Their report, entitled 'Focus on Pasifika Achievement in Reading Literacy', focuses on educational achievement in areas of reading literacy, mathematical literacy and scientific literacy. As in other OECD countries, the New Zealand results are based on "a representative sample of secondary school students aged from 15 years 3 months to 16 years 2 months" and "statistics from the PISA study are estimates of national performance based on a sample of students" (Comparative Education Research Unit, Ministry of Education, 2004). The scale used to observe reading literacy in the PISA measure was comprised of three subscales which were each designed to measure a particular aspect of reading literacy:

(a) retrieving information,

(b) interpreting texts, and

(c) reflection and evaluation.

Patterns of difference were found in the achievement levels of the main ethnic groups studied. In each literacy domain it was found that 
Pasifika students' achievement, on average, was significantly lower than the mean scores of Pakeha, Asian and Māori students. ... average achievement for Pasifika students was significantly lower than the OECD mean score.

(Comparative Education Research Unit, Ministry of Education, 2004, p.3)

Considerable diversity in performance was observed across the Pacific population, so much so that there was wider ranging performance within the Pacific population than between the Pacific and other populations. According to the report, the "larger proportion of Pasifika students at the lower end of the reading literacy scale is potentially a concern" (Comparative Education Research Unit, Ministry of Education, 2004, p.5). The large gap between the top percent and those who scored in the lowest levels was noted, and it was also noted with some concern that those students scoring below the lowest level "will likely have serious difficulty using their reading skills to gain knowledge and skills in other areas, increasing the risk that as school leavers they will lack qualifications and have difficulty finding work" $(2004$, p.6).

The PISA study also measured general issues related to learning including motivation and engagement, learning strategies, and student self-concepts, and the results were revealing. The PISA 2000 study identified factors that were associated with greater achievement in reading literacy measures. These were:

- being female

- being of multiple ethnicities

- being born in New Zealand

- usually speaking English at home.

High achievement among Pasifika students was found to be associated with:

- Student Motivation and Engagement

- Use of Learning Strategies 
- Student Self-Concepts

- Interest in reading, engagement in reading

- Time spent on homework and self-concept in computers

- Family-related factors including home educational resources and family wealth

- School-related factors including student-related behaviours, teacher shortages, teacher-related behaviours and the school physical infrastructure.

Particular differences were noted in the home and school circumstances of the low and the high achievers on the following:

- engagement in reading

- availability of home educational resources

- Principals' perceptions of student-related behaviours, teacher-related behaviours, and teacher shortages at their schools.

(Comparative Education Research Unit, Ministry of Education, 2004, p.20)

Although caution has been advised when interpreting these results, they would seem to suggest that these features may well be good predictors of Pacific student achievement in reading literacy.

In relation to the general experiences of Pacific students in higher education, statistics indicate that Pacific students (together with Maori students) have the lowest degree completion rates in New Zealand (Scott \& Smart, 2005). Dickie (n.d.) asks whether the performance of Pasifika students should "be considered purely in terms of technical skill or should it be looked at from the point of view of culture and ideology?" In this study I explore both the linguistic and the ideological nature of matches and mismatches between the reading demands of the first year of higher education, and the characteristics of the Pacific reader. The challenge lies in trying to observe the degree of fit 119 
between the ability levels of the students and the actual difficulty levels of the texts and the reading tasks required of the first year students. Having established whether potential mismatches in skill and ideology exist between the Pacific student and the tertiary institute, if mismatches are found to exist, it is then logical to develop and trial specific programmes aimed at addressing the issue. This study profiles Pacific readers and the texts and tasks within the context of the first year of university, seeking insights into the matches and mismatches that exist, and providing recommendations as to future directions.

\section{Chapter Summary}

This chapter has presented key findings from the review of relevant research literature. The following chapter presents the theoretical, conceptual and methodological frameworks that I have devised for this study as a result of the review of relevant literature. These frameworks are presented as one of the main contributions of this study to the endeavour to understand academic literacy at the higher levels in the New Zealand context, and they form the basis of the methodology of this research. 


\section{CHAPTER 3}

\section{THEORETICAL, CONCEPTUAL AND METHODOLOGICAL FRAMEWORKS OF THE STUDY}

\section{(3.1) Theoretical and Conceptual Frameworks of the Study}

First and second language reading is complex and multifaceted, and therefore requires a multifaceted approach to its investigation. The study design is intended to facilitate change by bringing about greater awareness, understanding, cooperation and better outcomes. Such change-oriented research "is more about a distinct set of goals than a unique approach to research or even a particular paradigmatic positioning" (O'Leary, 2004, p.133), and hence, influences from a number of methodological approaches may be seen in the modes of data collection and analysis in this study.

As Johnson and Saville-Troike (1992, p.602) note, quantitative and qualitative methods are "complementary rather than mutually exclusive", and while this study is primarily qualitative in nature (using interviews, observation, document analysis), it also employs "auxiliary quantitative procedures" (various tests and an inventory) for data types that necessitate this type of handling.

The research employed a multi-method approach with triangulation of data sources and an overarching emphasis on looking at the issues from as many angles as were feasible within the scope of the project. Such an approach in Applied Linguistics research places obvious practical, logistical and timerelated demands on the researcher (Johnson \& Saville-Troike, 1992:463). The multi-method approach is, however, best suited to this project wherein the 
qualitative and quantitative aspects work together to provide rich insights into this most complex entity called reading.

This is a pragmatic approach which echoes the sentiments of other researchers such as Larsen-Freeman and Long (1991, p.14), who point out that

[w] hat is important for researchers is not the choice of a priori paradigms, or methodologies, but rather to be clear about what the purpose of the study is and to match that purpose with the attributes most likely to accomplish it. Put another way, the methodological design should be determined by the research question.

Central to the study is the conceptualization of reading "not only as a mental individualistic process, but one that is also embedded in the sociocultural contexts in which it occurs", and the belief in the importance of taking a holistic perspective in carrying out research on reading (Davis, 1995, p.432). This approach seeks to investigate the issues by utilising methods that are designed from a holistic, emic perspective, with careful attention also being paid to ensuring credibility and dependability (Davis, 1995, p.436). Accepting reading as integrally combined with the cultures and contexts in which it occurs, carries the implication that studying literacy education as "cultural praxis" requires "interpretive, field-based methods of data collection, analysis, and reporting" (Florio-Ruane \& McVee, 2000, p.155).

A common criticism of qualitative studies relates to what many argue is the limited generalizability of such research (Johnson \& Saville-Troike, 1992; Davis 1995). This may be so, but as Davis (1995, p.441) points out, a strength of such studies is that they "allow for an understanding of what is specific to a particular group, that is, what cannot possibly be generalized within and across populations". In such cases it can be argued that the onus is firmly upon the reader of such interpretive qualitative studies to decide on whether, and the 
extent to which, that which is described in one research context applies to any other contexts and situations. Thus, the reader of this thesis must distinguish which of the insights contained herein may be applied for the betterment of their own individual and collective contexts and how.

I also agree with Prater's (2009) statement that the complexity of reading comprehension cannot be adequately represented by research methods that parse the component parts of reading skill and do not also take into account social, cultural and linguistic variables within the context of the reader, and this belief is fundamental to my thesis. Prater argues that researchers must work to develop research practices that seek to "untangle the intricate connections among the myriad of factors that impact academic achievement among English language learners while embracing the linguistic and sociocultural realities of English language learners". Accordingly this study was designed so that it constructively draws upon and draws together the three broad theoretical approaches to the study of reading and reading pedagogy within Applied Linguistics which have emerged over the past few decades. As mentioned in Chapter 2, these are (1) the componential, skills-based approach, (2) the cognitivist approach, (3) the socio-cultural approach.

Vacca (2002) provides a valuable description of these three major paradigmatic shifts in the study and teaching of reading, detailing how research within the 'Reading and Study Skills Paradigm' (1900s to 1960s) included investigation into and identification of the reading and study skills associated with content area reading in the various content areas. The 'Cognitive and Learning Paradigm' (1970s through 1980s) saw investigations into the cognitive and metacognitive processes in reading with schema theory, text structure, metacognition, strategic learning all having an influence on reading research in that period. More recently the 'Social Constructivist 
Paradigm' (1990s to current), which argues that learners construct knowledge inside their minds, has prompted a research shift towards investigation of how the social context of the classroom affects the way those within it interact. Consequently, greater research effort was directed towards the experiences, views and beliefs of students and teachers, and to the link between reading and learning.

Drawing upon the three paradigms above, in this thesis project the componential, skills-based approach underlies the framework by which I examine the Reader characteristics. The cognitivist approach is reflected in the close observations of the interactions between Reader and Text and meaning-making processes utilized during the Free Recall exercises. While the socio-cultural approach underlies much of the project's belief that social and cultural contexts are very much a part of the holistic lens through which reading, what is read, how it is read, and who reads it in the first year of university, need to be viewed and investigated.

Central to the study is also the utilization of Case Study methodology. In this study the cases - the University, the first year Courses, and the students within those Courses - are embedded within each other. They are studied not only for their intrinsic value, but also for their instrumental value in accomplishing the goals of the study, and for their collective value in allowing us to find important connections between the individual cases and to better understand the integrated systems (Stake, 1995, p.2). Stake (1995, p.12) emphasizes that as primarily qualitative researchers "[w]e try hard to understand how the actors, the people being studied see things ... the qualitative case researcher tries to preserve the multiple realities, the different and even contradictory views of what is happening" (Stake, 1995, p.12). This advice has underpinned the research and analysis in this thesis project. 


\section{(3.2) Methodological Frameworks Designed for this Study}

Due to the unique nature of this study's context and participants, it was necessary to devise or adapt methodological frameworks more specifically suited to the study context than were available in current literature. Thus, from the review of literature and with consideration given to the nature of the context and participants, the following frameworks were designed and used to guide the collection and analyses of data. The frameworks include an overarching study design framework for understanding academic reading literacy in higher education, and two further frameworks for the study of the nature of the academic Texts and the academic Readers respectively. These are outlined in the following sections.

\section{(3.2.1) The Reader-Text-Task Framework for Investigation of Academic Reading in Higher Education}

While the Pacific Readers themselves are undoubtedly the centre of this research, gaining an understanding of the context in which they operate is essential to an authentic understanding of them. Readers are thus best understood in relation to the texts they are reading, and the tasks they are required to carry out with these texts within that specific socio-cultural context.

The understanding around which the methodology of the research is built is that reading (and specifically academic reading) is a dynamic process involving the interplay between the Reader's own characteristics and the characteristics and features of their context (specifically the texts and tasks that exist within it). These inherently complex entities - Reader, Text and Task also interact in ways that are shaped by the unique combinations in which they exist in any particular reading scenario at any particular time. This framework essentially constitutes my thesis and informs the three-part design of the study, 
and all results presented herein are drawn from this framework and provide illustrations of the thesis at work in a particular context. While I propose that this fluid conceptualization of reading allows us to more accurately represent the nature of academic reading in educational contexts, at the same time it does present its own very unique challenges to research. With these three factors each being comprised of multiple features themselves, it was necessary to limit this research project to the investigation of a selected sub-set of these factors.

The crucial link between the Text, Task and Reader is by no means 'new', having been discussed in various other forms by researchers over the past decade, but its treatment has been mostly from a theoretical rather than a practical standpoint. Reading is acknowledged as a process shaped partly by the text, partly by the reader's background, and partly by the situation the reading occurs in (Hunt, 2004, p.137, cited in Hermida, 2009, p.4). In an article on college (university) study, Nist and Simpson (2000, p.2) discuss the external factors that influence reading and studying, and specify that these "external factors include the texts that are assigned and the academic tasks that are either tacitly or explicitly communicated by the professor". They go on to discuss the role of the text and the role of the academic task in influencing active and effective study and stress that "researchers need to know more about how factors such as text, academic task, and students' beliefs affect and interact with strategy use" (Nist \& Simpson, 2003, p.10).

Meyer (2003, p.204) also explicitly works with the three central factors of text, task and reader but also adds a further factor called 'strategy variables' when explaining the variables involved in reading comprehension. Meyer's (2003, p.205) comprehensive model diagrams the interactions between reader, text, task and strategy variables for the task of reading computer-cued reading 126 
material. Each of the text, task, reader and strategy variables are themselves composed of many complex variables. The ones identified explicitly by Meyer (2003, p.205) are as follows:

Table 3.1: Meyer's (2003) reader, text, task and strategy variables

\begin{tabular}{|c|c|c|c|}
\hline Reader variables & Text variables & Task variables & Strategy variables \\
\hline $\begin{array}{l}\text { Verbal ability \& } \\
\text { Word Knowledge }\end{array}$ & Text structure & $\begin{array}{l}\text { Mode or Rate of } \\
\text { Presentation }\end{array}$ & Structure Strategy \\
\hline Education & $\begin{array}{l}\text { Topic Content \& } \\
\text { Word Familiarity }\end{array}$ & Response Mode & Rereading \\
\hline Age & Signaling & $\begin{array}{l}\text { Task } \\
\text { Requirements }\end{array}$ & Underlining \\
\hline World Knowledge & Cohesion & $\begin{array}{l}\text { Type and Setting } \\
\text { of Task }\end{array}$ & and so forth... \\
\hline Perspectives & $\begin{array}{l}\text { Number of Levels } \\
\text { in Structure }\end{array}$ & and so forth... & \\
\hline Values & $\begin{array}{l}\text { Amount and Type } \\
\text { of Details }\end{array}$ & & \\
\hline Norms & Sentence Length & & \\
\hline Reading Expertise & $\begin{array}{l}\text { Left-branching } \\
\text { Sentences }\end{array}$ & & \\
\hline $\begin{array}{l}\text { Working memory } \\
\text { Capacity }\end{array}$ & Genre & & \\
\hline Styles & Typography & & \\
\hline Interests & and so forth... & & \\
\hline and so forth... & & & \\
\hline
\end{tabular}

Meyer's approach has informed many aspects of my work.

Text, task and reader factors are also drawn together in the work of Horiba (2000), who investigated the effects of reader language competence, text type and task on the concept of reader control in reading. Reader control is seen as the ability of competent readers to flexibly control their own text processing in order to fulfill their own comprehension goals in that context, such control is undoubtedly related to metacognition and is primarily demonstrated in efficient processing of text. According to Horiba (2000, p.224), readers who 
demonstrate this ability "can and do regulate their cognitive processing operations according to the goal of their reading."

Horiba's (2000) study of the way that native and non-native English speakers processed text (observed through think-alouds) provides some evidence for the effect of task type and reader language status on text processing. Furthermore, patterns were found in the way the nature of the text affected the readers' processing during reading, and it was suggested that different text types within one wide genre (such as expository text) "may invite different patterns of processing" (Horiba, 2000, p.255). Horiba's results are important in highlighting how complex and intertwined the influences of the text type and task type are with the characteristics of the reader. Horiba's (2000, p.258) recommendation for future research is that it should investigate "the effect of type of task on text processing and learning by keeping in mind that both the type of text and the type of task both potentially affect processing and memory".

Koda (2005) also makes reference to the crucial combination of text, task and reader in reading research, as does Snow (2002, p.11, cited in TEC, Learning Progressions Background, n.d.), who describes how 'comprehension' entails three elements:

1) The reader who is doing the comprehending

2) The text that is to be comprehended

3) The activity in which comprehension is a part.

However my contribution is the unique configuration of the factors within these three elements, and the belief is that acknowledgement of a further factor is vital, and this is that the three dimensions of text, task and reader occur within a larger socio-cultural context, and this context in turn also interacts 
with and shapes each of the three elements. This forms the framework that comprises the thesis of my research and is represented as shown below:

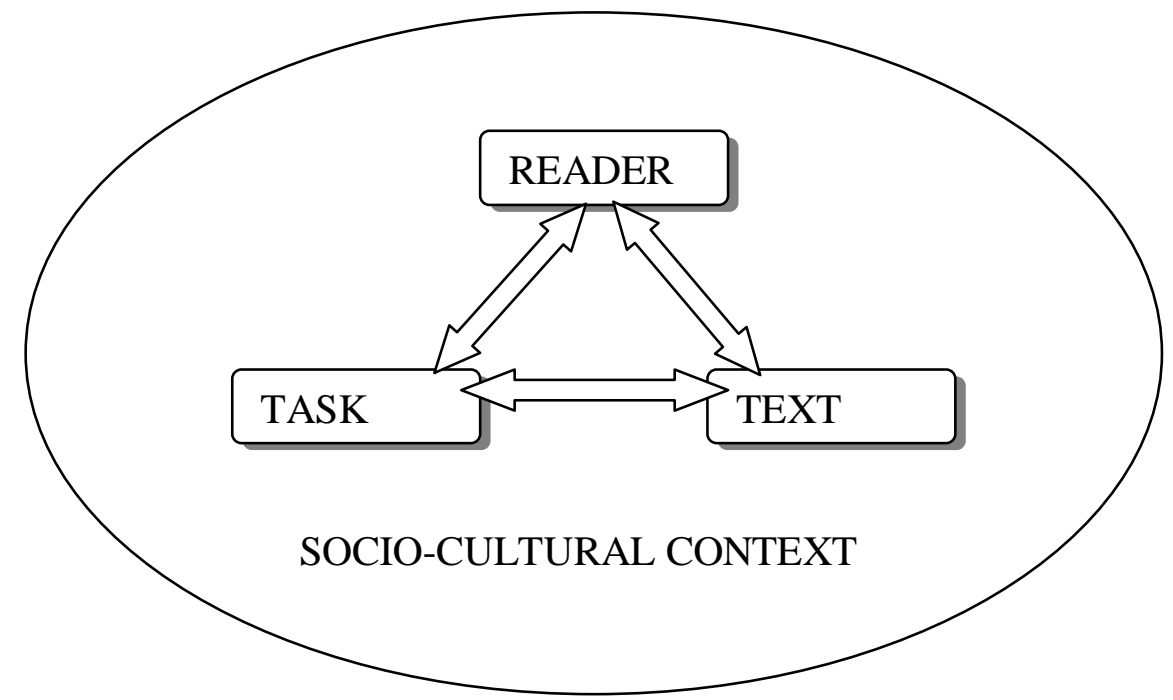

Figure 3.1: The Reader-Text-Task conceptual framework for investigation of academic reading in higher education

\section{(3.2.2) Framework for the Investigation and Profiling of the Linguistic and Non-verbal Characteristics of Academic Texts in Higher Education}

In an attempt to devise a practicable framework for investigating the nature (and hence the demands) of authentic academic texts in the first year of university study, I have developed a three-part framework for the systematic investigation of the features of first year texts by compiling the factors found in the literature to be of significance to text readability. 
Table 3.2: Framework for the investigation of the linguistic and nonlinguistic features of text

\begin{tabular}{|c|c|c|c|c|c|}
\hline \multicolumn{2}{|c|}{$\begin{array}{l}\text { Lexical \& Syntactic } \\
\text { Complexity }\end{array}$} & \multicolumn{2}{|c|}{ Idea Density \& Structure } & \multicolumn{2}{|c|}{ Format \& Design Features } \\
\hline construct & measure & construct & measure & construct & Measure \\
\hline $\begin{array}{l}\text { Vocabulary } \\
\text { profile of texts }\end{array}$ & $\begin{array}{l}\text { Lexical } \\
\text { profiling } \\
\text { (using } \\
\text { software) }\end{array}$ & Idea density & $\begin{array}{l}\text { Analysis of text } \\
\text { for propositions } \\
\text { or 'Idea Units' }\end{array}$ & $\begin{array}{l}\text { Graphic } \\
\text { Conventions }\end{array}$ & $\begin{array}{l}\text { Analysis of } \\
\text { layout, } \\
\text { punctuation, } \\
\text { typeface, } \\
\text { symbols, etc. }\end{array}$ \\
\hline \multirow[t]{2}{*}{$\begin{array}{l}\text { Syntactic } \\
\text { Features \& } \\
\text { Complexity }\end{array}$} & $\begin{array}{l}\text { Analysis of } \\
\text { text samples } \\
\text { or use of } \\
\text { appropriate } \\
\text { software }\end{array}$ & $\begin{array}{l}\text { Idea } \\
\text { structure }\end{array}$ & $\begin{array}{l}\text { Analysis of } \\
\text { text's } \\
\text { rhetorical } \\
\text { structure / } \\
\text { 'propositional } \\
\text { textbase' }\end{array}$ & $\begin{array}{l}\text { Reference } \\
\text { Apparatus }\end{array}$ & $\begin{array}{l}\text { Analysis of } \\
\text { titles, index, } \\
\text { introductions, } \\
\text { abstracts, } \\
\text { summaries, } \\
\text { etc. }\end{array}$ \\
\hline & & Coherence & $\begin{array}{l}\text { Analysis of } \\
\text { cohesive } \\
\text { devices }\end{array}$ & $\begin{array}{l}\text { Non-Verbal } \\
\text { Information }\end{array}$ & $\begin{array}{l}\text { Analysis of } \\
\text { maps, } \\
\text { diagrams, } \\
\text { etc. }\end{array}$ \\
\hline
\end{tabular}

As mentioned before, scope does not permit that all of these textual features be investigated in this study. However this framework is proposed as a means for future systematic analysis of the characteristics of academic text in higher education for the purposes of ascertaining the nature and degree of challenge presented by authentic texts across various disciplines within the university.

It is proposed that this knowledge, when combined with knowledge ascertained regarding the nature of the tasks required of readers within these disciplines, and the nature of the readers themselves, will inform appropriate and strategic responses to the development of academic reading literacy in the first and subsequent years of higher education. Moreover, it is anticipated to have useful application in the areas of text-book authoring and development, 
and also be of use in informing work on facilitating the transition from the text experiences of upper secondary to tertiary education.

Of the features in the framework, this study seeks to document the lexical and conceptual density, the vocabulary profiles, and the idea density of the text samples are explored, and a proxy measure of sentence length is used to gain a picture of the syntactic complexity of the texts in the first year courses.

\section{(3.2.3) Framework for the Holistic Profiling of academic Readers' Characteristics in the First Year of Higher Education}

For the purpose of the study a framework for profiling readers using a componential, interactive-compensatory, and socio-cultural view of reading was created. While I acknowledge that there are drawbacks to both the composite skills approach (small scale studies, inconsistent outcomes, variability of measures used and conditions and the tendency to identify learners as 'at risk') and the socio-cultural view (again, small scale studies, questions about the generalisability of results to other populations and the reliability of measures used) (Prater, 2009), their combination does nonetheless enable the holistic approach that I feel is vital to the study of academic reading.

Contemporary reading comprehension models say that the reader uses a combination of (1) background knowledge, (2) linguistic knowledge and (3) text knowledge to comprehend, and that meaningful comprehension cannot occur without all three (Pretorius, 2006). By thematically analyzing existing relevant literature I have identified and selected broad yet discrete component categories that fall within Pretorius' key categories and are central to academic reading ability. I have then combined these to create a framework by which the systematic and holistic profiling of academic readers may be undertaken. 
The framework comprises 7 broad categories into which the key factors, skills and components I encountered in my literature review fit.

I propose that through the combined investigation of the factors in this framework one can produce richly descriptive and informative profiles of academic readers within the investigated context. Through the holistic profiling of reader abilities, traits, characteristics and beliefs, and through the analysis and documentation of the factors that appear to be related to difficulties and breakdowns in academic reading, one can reach the informed position from which to support and develop students in academic reading in higher education.

The framework which draws together a holistic series of key factors influential upon academic reading has also been informed by the InteractiveCompensatory Model of Learning (ICML) proposed by Stanovich (1980). Since my list of 'component skills' extends well beyond the traditional understandings and parameters of the cognitive, I have chosen to use the term 'influential factors' rather than simply 'skills' because the factors that influence the SL reading process for Pacific readers, as I see it, are by no means limited to 'skills' alone. The framework is presented below: 


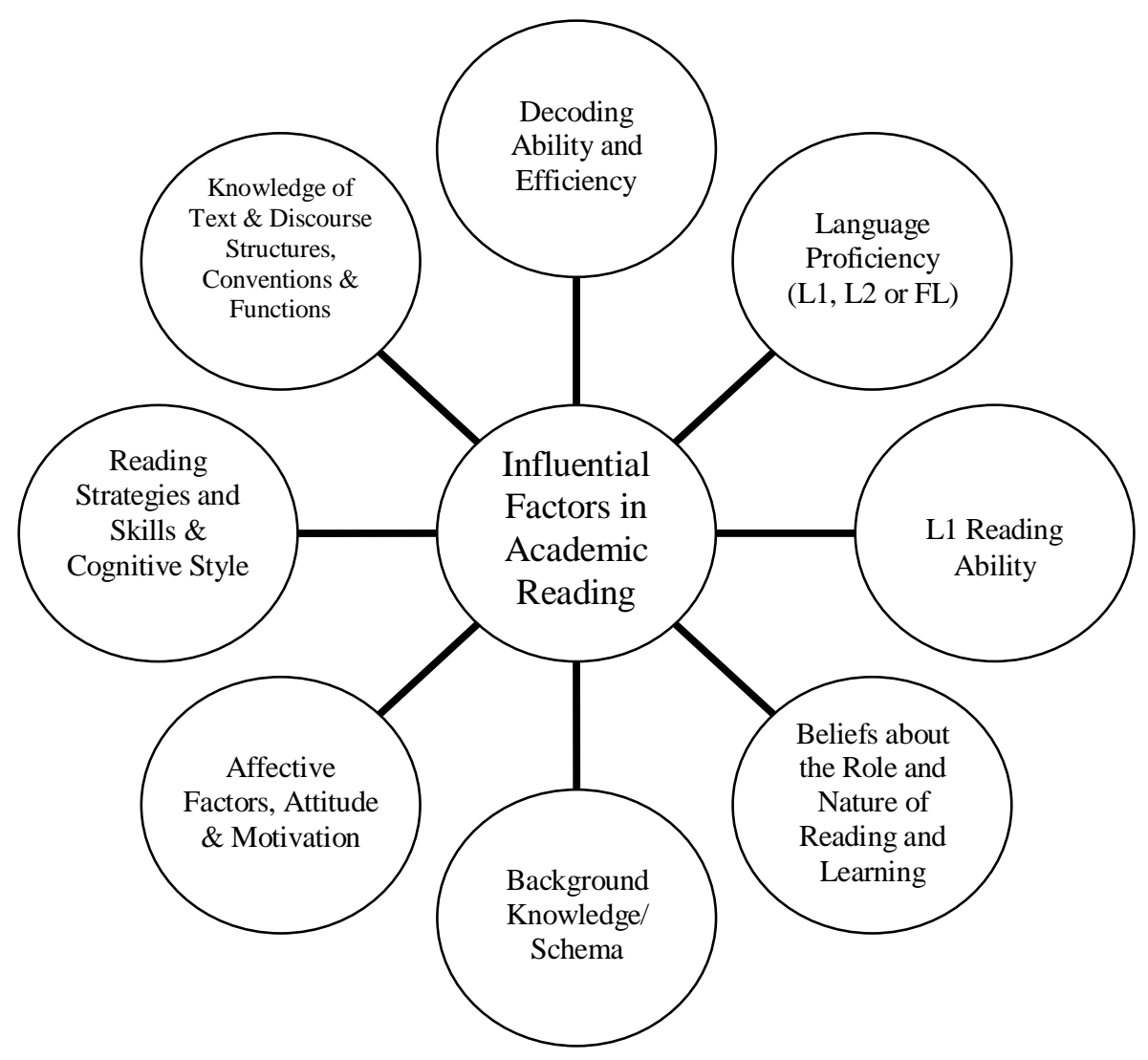

Figure 3.2: Influential factors in academic reading: A framework for reader profiling

Overall, this study will attempt to gain an holistic view of the Pacific student, eliciting rich data to build up their academic reading profiles. However, as with the text analysis framework, it is impractical to address all aspects of the framework in this study, so certain key features have been selected for investigation in this work.

\section{(3.2.4) Pacific Metaphorical Framework for the Project Design}

This project also coincides with signs of a 'coming of age' of Pacific research, Pacific epistemologies, Pacific methodologies, and a growing feeling amongst 
researchers of Pacific descent involved in researching the issues of Pacific peoples that it is time for recognition and validation of the knowledge systems and ways of thinking that characterize Pacific peoples. Accordingly, an important part of this project is the Pacific research framework that underpins the study and the manner in which the various parts of the project are integrated. Several key research frameworks frequently referred to in literature and research concerning Pacific peoples are the 'kakala' model (Helu-Thaman, 1992), Fa'afaletui (Tamasese et al., 1997), Tivaevae (MauaHodges, 2000) and the Vanua (Nabobo-Baba, 2006) (all cited in HeluThaman, 2009, p.5). Common to many such Pacific frameworks is the powerful use of metaphor, and many comprise a central metaphor through which the research process is conceptualized and which brings unity and clarity both to the issue itself and to the task and processes involved in investigating it.

The metaphorical framework upon which this research project was designed is that of the woven pandanus fibre mat and the weaving process by which it is created.

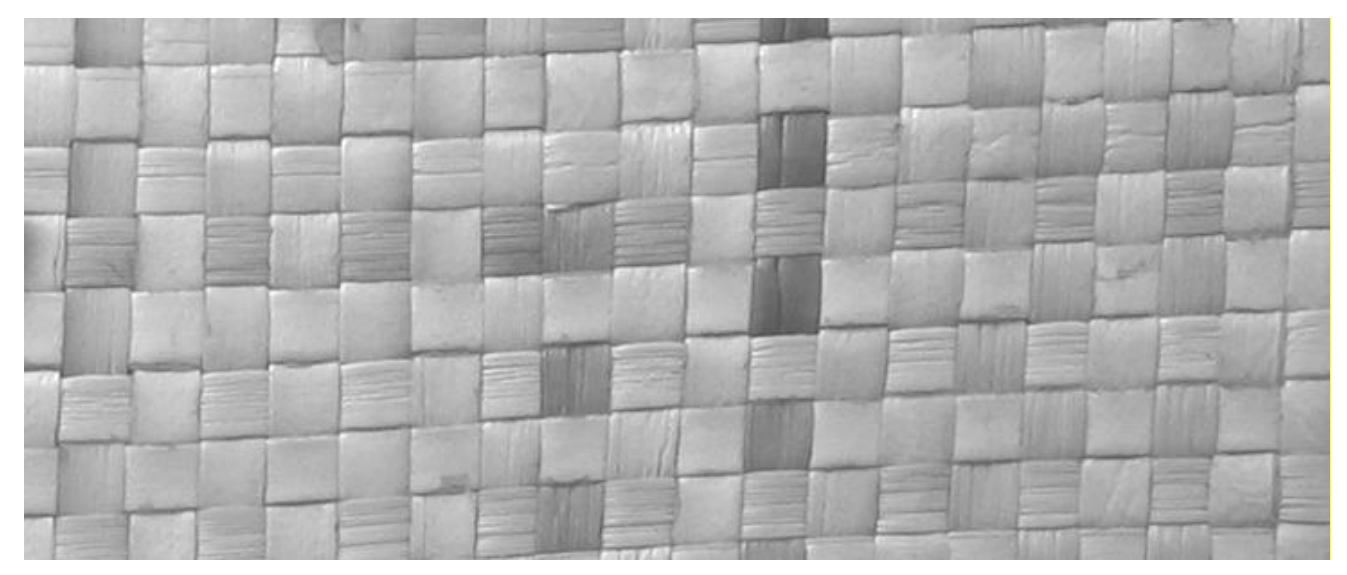

Figure 3.3: Woven Pandanus fibre mat 
This framework has assisted me to maintain clarity and control of a complex and multi-faceted research project in which multiple and multi-part 'strands' were simultaneously collected and analysed. In this metaphorical framework, just as the best individual Pandanus fronds are selected and laboriously prepared, cut to size, and carefully woven into the mat, so too have the various holistically representative strands of this research project been individually prepared. The weaving of these strands together is symbolic of the systematic cross-tabulation that forms a large part of this study. Once prepared, the strands are then 'woven' together with the other strands to construct a weaving that will, like a woven mat which forms the basis of the family home, constitute a research foundation upon which future research and discussion may be situated, located and based. In this way the strands, while of considerable interest on their own, take on new value when woven together, and it is often only when the final strands are in place that one may appreciate the full design of the weaving.

\section{Chapter Summary}

Thus, elements of all the frameworks mentioned in this section have been harnessed for the insights they are able to provide, and for their contribution to developing a holistic understanding of academic reading in this context. Ultimately, however, whatever methods are chosen in research such as this, what is most important is that they ensure that the final research outcomes are of clear benefit to Pacific students, the higher education institutions in which they are enrolled, and the concerns of the communities they represent. In the following chapter the expanded research questions derived from the three part framework are presented, along with an overview of the participants, setting, ethical considerations and research methods, instruments and procedures. 


\section{CHAPTER 4}

\section{METHODOLOGY}

This chapter presents the Research Questions that have guided this study, the methodological framework and methods designed for this study by which data was collected and analyzed. On the whole, my research aims to holistically profile first year student readers of Pacific descent and their academic reading context, and the ways in which these elements interact to shape the Pacific Readers' experiences of academic reading in their first year of study at the New Zealand University.

The sections of this chapter are as follows:

(4.1) Research Questions

(4.2) Research Methods: Overview and Rationale

(4.3) Ethical considerations of the study

(4.5) Research Setting and Participants

(4.6) Methods of Data Collection and Analysis: Research Instruments and Procedures.

\section{(4.1) Research Questions}

The review of the research literature provided a guide to the expanding of the original three Research Questions presented in Chapter 1 to include a number of specific sub questions. Together, the questions and sub-questions guided the choice of methodology in this research. They are as follows: 
Research Question One:

What is the nature of the typical academic texts encountered in the first year of university study?

(a) What are the key linguistic characteristics of typical authentic first year academic texts in the chosen Faculty in terms of their

(i) lexical profile?

(ii) grammatical complexity?

(iii) conceptual density?

\section{Research Question Two:}

What is the nature of the typical academic reading tasks required of the reader in the first year of university study?

(a) What types of reading tasks/activities/outcomes/processes are required in typical first year courses?

(b) In a typical first year course how do the reading task representations of lecturers and students compare?

(c) Given the characteristics of the texts and tasks, what are the characteristics of a 'good reader' in this Faculty?

\section{Research Question Three:}

What are the characteristics of Pacific students as academic readers in the first year of university study?

(a) What are the characteristics of first year Pacific students in terms of:

(i) Salient reading experiences throughout their reading history (Sociocultural and literacy background)?

(ii) Vocabulary size (as an aspect of language proficiency related to academic reading)?

(iii) Reported patterns of reading strategy use, habits and attitudes?

(iv) Reading rate (as an indication of fluency)?

(v) Affective and perceptive responses to text?

(b) What is the nature of the Pacific students' interactions with academic text whilst performing a typical reading task with authentic texts from their Course?

(c) What matches and mismatches exist between the academic reading profiles of the Pacific first year readers and the typical 'good reader' in this discipline?

(d) Is there a link between academic reading profile characteristics and academic success? 
The Research Questions in expanded form seek to holistically profile both the readers and their context and ultimately provide an indication of the degree of 'fit' between the two.

\section{(4.2) Research Methods: Overview and Rationale}

As indicted previously, this thesis reports on a project that was multi-method and holistic in its approach. The use of multi-methods and holism for me has been primarily driven by 1) the belief that a construct this complex necessitates such a research response, and 2) the vital importance of holism as a guiding principal in research endeavour in Pacific and other indigenous worldviews (Kovach, 2009).

Reading is a multi-dimensional entity and each of the facets investigated warrants its own means of data collection and analysis. This study comprised a combination of text analysis procedures, interviews, document analysis, observation, vocabulary testing, verbal report protocols, free recall protocols, and inventory based self-rating. The data analysis techniques comprised both analytical and qualitative techniques and the exploratory nature of the study necessitated both open and closed questioning, and thematic data analysis.

Following Lincoln and Guba (1985, p.109) and O'Leary (2004, p.115), the credibility of this predominantly qualitative research project was sought by:

- collecting rich data,

- attempting broad representation,

- building trust and rapport through prolonged engagement,

- triangulation of data sources,

- participant-checking of findings,

- providing description of my methods to allow scrutiny/replication 
Ajideh (2003) holds that there are two main understandings of reading. The first is a "product-oriented approach" which "assumes meaning exists in the text itself, and it is text-based factors that determine meaning" (2003, p.1). The second is a "process-oriented approach" in which "meaning is obtained through a successful interaction between the reader and the text, and it is inside-the-head factors that play an important role in comprehension" (Ajideh, 2003, p.1). Ajideh argues that research so far has tended to focus more on the product of reading than the process. There are several clear advantages of a process-oriented approach to understanding reading. The first is that investigating reading processes helps to distinguish between what different readers do when they are both given the same text (product). Secondly, having observed and defined the processes of the more successful readers, a persuasive argument stands that these processes could be taught to other learners or less able students in order to assist them to improve their reading (Ajideh, 2003, p.2).

Block (1986, p.464) also believes there is a clear need for "process-oriented research" in order to shed more light on what readers actually do, and for this to ultimately inform decision-making by educators. To gather processoriented descriptions there is a wide array of techniques which fall largely into two main categories: (1) reports of reading processes obtained during a reading event through such methods as the think-aloud protocol, and (2) information gained about the reading process after the reading event through methods such as the retrospective interview or reflection.

Verbal reports from Reader participants therefore seem a useful way to gain insights into the nature of their meaning-making processes, and cognitive and affective responses to text. The relatively extensive use of verbal reports and protocol analysis in reading research in the last decade is due to belief in their 
ability to yield "rich and compelling data" (Afflerbach, 2000, p.164). The potential advantages of the verbal report include the following:

- they provide access to the constructive and responsive processes that comprise reading, allowing us to further our understanding of the complex constructs involved in reading that we would otherwise only be able to investigate indirectly.

- they provide a lens on both the traditional constructs of reading, and important reader characteristics such as motivation and affect. They are particularly useful for exploring the relationship of these factors to the traditional constructs of cognition.

- they also allow for investigation of the influence that contextual variables such as text, task, setting and reader ability, have on reading.

(Afflerbach, 2000, p.165)

Protocol analysis provides evidence that constructive cognition is at the heart of reading, but it also shows that reading is more than just cognition. A significant advantage is that the analysis of protocols serves to "contribute to the initial building of theories that represent progress in the understanding of reading". These theories can then "help us chart a course of the work that remains to fill the gaps of this understanding" (Afflerbach, 2000, p.165).

However, there have been some criticisms of the verbal report. These challenges stem primarily from questions relating to the validity of the protocol and the fact that any lapses in the "integrity" of the method will influence the quality of the data obtained through them (Afflerbach, 2000, p.165). An obvious drawback is that the method does rely on the participants' ability to verbalize what they experienced during their interaction with the text, and that this verbalization can be influenced by a number of things, including "the relationship between the participant and the researcher, gender 
differences between subject and researcher, cultural differences in reporting and using language, or differences in how the subject conceptualizes his or her role as a reporter of reading phenomena" (Afflerbach, 2000, p.173).

Nevertheless, the consistent and widespread usage of the verbal report and protocol analysis within reading research in the last decade would indicate that it has been broadly accepted as a bona fide method for reading research, provided that it is administered and carried out consistently and conscientiously. The verbal report is not only suited to studies involving a single focus, but is valuable for (1) providing insights into the "totality of the reading task" (Afflerbach, 2000, p.167), and (2) bringing to light insights into both the cognitive and important non-cognitive aspects of academic reading. This makes it ideal for use in this study. Of additional benefit to participants is that it most often has the effect of developing metacognitive ability and self awareness within the reader. This is another reason why, given the place of the Pacific value of 'reciprocity' at the heart of this study, it is an appropriate method for this study.

An acceptance of verbal reports as one of the study's means of gaining insights into the processes and experiences of readers, then required a decision about the question of what type of verbal report to utilize. According to Block (1986, p.464)

process-oriented descriptions are usually obtained through two types of verbal reports: (a) retrospective, obtained after the reading task is completed, or (b) introspective reports and think-alouds, obtained during reading. Retrospective reports have the advantage of keeping the process intact and the potential disadvantages of being distorted or inaccurate (Hare, 1981). 
Difficulties encountered with the use of think-alouds in earlier research (Davidson-Toumu'a, 2005) also led me to choose retrospective interviews and Free Recall as the main means of data collection. The drawback of retrospective interviews is that it is indirect, does not occur at the moment of processing, and thus potentially misses out on some aspects of the 'why' and 'how' of the reading experience. Despite this, a clear advantage of the retrospective report as a data collection method is that the technique allows the reading process to stay "intact” (Block, 1986, p.464).

With respect to data analysis, this study reflects the view that each researcher needs, "through experience and reflection, to find the forms of analysis that work for him or her", with "[t]he nature of the study, the focus of the research, the curiosities of the researcher" determining the specific analytic strategies utilized. These factors also determine the balance between "categorical aggregation or direct interpretation" (Stake, 1995, p.77). In this study a multimethod data collection approach has necessitated a similarly multi-method approach to data analysis, however despite a variety of strategies being devised and utilized, the forms of qualitative analysis all followed the generally accepted qualitative data analysis procedural steps of (1) data reduction (the reduction of large amounts of data into neat and usable forms), (2) data display (the "organized compressed assembly of information that permits conclusion drawing") and (3) the drawing and verification of conclusions (Miles, 1994, p.9).

\section{(4.3) Ethical Considerations}

Approval for the project was sought from the Human Ethics Committee of Victoria University of Wellington and was granted on June 30, 2008. In the research design considerable care was taken concerning the following. 


\section{(4.3.1) Ensuring Minimal Disruption to Participants' Normal Working/Studying Routines and Workloads.}

Every care was taken to only meet during times that the participants felt that they were able to. Data collection exercises took place in our scheduled meetings over a period of one trimester (12 weeks) or until all data was collected. By spreading the load of data collection over the trimester the amount of disruption to normal study routines was minimized. Likewise, great care was taken with Lecturers and Tutors to ensure that meetings did not take place during times of high workload, and meeting times were always negotiated and decided on by the participant. As with the student participants, virtually no 'take home' elements were necessary, thus making sure that the important criterion of ensuring minimum extra workload for participants was achieved (Davis 1995, p.442).

\section{(4.3.2) Maintaining and Protecting Participant Anonymity.}

A crucial ethical issue in the study was ensuring that the anonymity of the participants was preserved. In this type of qualitative research methodology the protection of the participants' welfare and anonymity is key (Davis 1995, p.442). Given that I was simultaneously involving students and teaching staff from the same Courses, the identity of the students was never made known to the Lecturers. For my own purposes students were given a simple numbering system for identification (S1, S2, S3, and so forth), and information on gender and place of birth was also added to the number (F or M for Female and Male, and $\mathrm{P}$ to signify being Pacific born and educated). While I felt that this numbering system was not particularly culturally appropriate, it was necessary to strike the balance between the depersonalising of my readers and their need for anonymity. 
For protection of staff identity the participants are simply referred to as Lecturer or Tutor, and any reference to gender has been removed. Since some staff members' involvement in the project was subject to their anonymity being preserved, in analysis of their Course Outlines care has been taken to remove all content references and replace them with generic terms and to not directly identify the School or tertiary Institution to which they belong.

In research of this kind it would usually be desirable for the patterns of "behaviour, beliefs, and/or values" to be presented and reported as aggregate data to help ensure anonymity (Davis 1995, p.442). While this is so for the Reader description chapter in which general trends are presented, I have chosen to also provide case study descriptions of specific students in order to demonstrate the value of the holistic profiling system in action, but in doing so care has been taken to protect participant identity.

\section{(4.3.3) Upholding Core Pacific Values.}

Being a study undertaken with and for Pacific peoples, it was vital that Pacific values were upheld throughout. Anae, Coxon, Mara, Wendt-Samu and Finau's (2001) documentation of 'Pasifika Research protocols' is widely referred to as being a clear and concise encapsulation of the core values and behaviours considered appropriate when carrying out research with and for Pacific peoples. A subsequent publication by the Health Research Council (HRC) (2004) laid down similar "guiding principles" for Pacific research. These central values which guide decisions in planning, conducting, analyzing, and distributing information from the study are:

- Respect

- Cultural competency

- Meaningful engagement

- Reciprocity 
- Capacity building (HRC, 2004, p.2).

Throughout the project these values have guided interactions between myself as researcher and the participants.

Particular attention has been given to the value of reciprocity. This concept is not only of core importance to Pacific societies, it is also recognized as a sound research practice in Western frameworks, particularly those involving qualitative data collection within a social sciences tradition. For example, Davis (1995, p.443) documents "what is commonly referred to in the ethnographic literature as exchange of services or reciprocity (e.g., Agar, 1980)", which is based on the understanding that "participants are entitled to some kind of return for the time and effort they contribute to the study". Core to the research approach adopted in this study is the upholding of these core Pacific values in research practice, relationship building, and a commitment to the "well-being and empowerment" of those involved in the study and those for whom the study's outcomes are relevant (Airini, Curtis, Johnson, Luatua, O’Shea, Rakena, Reynolds, Sauni, Smith, Huirua, Tarawa \& Ulugia-Pua, 2009, p.7).

\section{(4.4) Research Setting and Participants}

To enable the outcomes of the research to be of more immediate, specific and practical effect I have deliberately chosen to focus on readers in 'mainstream' academic reading situations within a NZ University, rather than to focus on specifically language-oriented or preparatory-type university courses. I had originally intended to sample several of the Faculties which traditionally record high percentages of Pacific student enrolment, however the scope of the project and my desire to collect particularly rich data led me to 'zoom in' on just a handful of first year Courses within the two faculties of (1) Humanities and (2) Commerce within the chosen New Zealand University. Additionally, I 
concentrate on one particular first year Course (Course A) for the more detailed observations of the Text, Task and Reader interaction, and 'zoom' in even further on particular readers within this Course.

I have endeavoured to include both New Zealand and Pacific nation-born and educated students who were in their first year of university study and to achieve as much of a gender and ethnic balance as was possible through the use of culturally appropriate participant recruitment procedures. While this is not a comparative study, for the estimation of first year student vocabulary size a non-Pacific student group was involved to provide a basis for comparison of the Pacific students in the study with their non-Pacific peers.

\section{(4.4.1) Participant Recruitment Procedures}

In terms of recruitment of participants in Pacific research, Anae et al. (2001, p.36) point out that it is more successful to "involve processes that draw from researcher, community, and/or institutional networks". By the use of such means of recruitment I was able to successfully negotiate site entry and participation of people within the research context.

The Humanities and the Commerce Faculty first year Courses were recruited in the following manner: firstly, mainstream courses with traditionally high Pacific student enrolments were identified from enrolment statistics, and Lecturers were identified using the University's website. Secondly, personal network connections were utilized to facilitate introduction to the Lecturers, Tutors and Course coordinators who would later become involved in the study.

Once the four main case study Courses were identified and Lecturer/Tutor involvement secured, lists of students who had self-identified upon enrolment 
as being of Pacific descent were generated by the administrative coordinators of the Schools who forwarded my introductory email to the students on this generated mailing list. The email briefly explained the study and salient ethical issues and invited response directly to me. Uptake in the Commerce course was very encouraging, but in the Humanities papers it was markedly less so.

Student participants were clearly advised that they were free to withdraw from the study up to a certain point in the data collection process. I therefore anticipated a certain degree of attrition. While only 12 students feature in this thesis, there were originally more than 19 students who volunteered to participate but who, just prior to or in the course of the study, withdrew either formally or informally. Their data are not complete enough to form individual profiles and are thus utilized in aggregate form where relevant.

The first year students of Pacific, Asian and other descent who participated in the vocabulary measure to provide a basis of comparison were recruited from several large first year papers within the University. After receiving permission from the lecturers, a class visit and sign-up sheet and follow up email were used to obtain these participants' vocabulary scores. In return for their participation they were given feedback on their vocabulary scores if they indicated the wish to receive it.

\section{(4.4.2) The Student Participants}

The following table details the Pacific Student Readers involved in this study: 
Table 4.1: The Pacific student reader details

\begin{tabular}{|l|l|l|l|l|}
\hline $\begin{array}{l}\text { Student } \\
\text { Code } \\
\text { in Study }\end{array}$ & Age & Sex & $\begin{array}{l}\text { Pacific Ethnicity } \\
\text { Self-identified with }\end{array}$ & Course Taken \\
\hline S1(M) & $18-25$ yrs & M & Samoan & Course C \\
\hline S4(M) & $18-25$ yrs & M & Fijian & Course A \\
\hline S5(M) & $18-25$ yrs & M & Niuean & Course A \\
\hline S7(F) & $18-25$ yrs & F & Samoan & Course A \\
\hline S9(F) & $18-25$ yrs & F & Samoan & Course A \\
\hline S12(M) & $18-25$ yrs & M & Samoan & Course A \\
\hline S13(PF) & $25+$ yrs & F & Samoan & Course A \\
\hline S14(F) & $18-25$ yrs & F & Tokelauan & Course B \\
\hline S15(F) & $18-25$ yrs & F & Samoan & Course B \\
\hline S17(PF) & $18-25$ yrs & F & Samoan & Course D \\
\hline S18(PF) & $18-25$ yrs & F & Tokelauan & Course E \\
\hline S19(M) & $18-25$ yrs & M & Tongan & Course F \\
\hline
\end{tabular}

\section{(4.4.3) The Case Study Courses}

The following first year Trimester 1 Courses were case studies in this project:

Table 4.2: The case study courses involved in the study

\begin{tabular}{|l|l|l|}
\hline Course Title in Study & Faculty & $\begin{array}{l}\text { \# Pacific Readers } \\
\text { recruited from Course }\end{array}$ \\
\hline Course A & Commerce & 6 \\
\hline Course B & Arts & 2 \\
\hline Course C & Arts & 1 \\
\hline Course D & Arts & 1 \\
\hline Course E & Arts & 1 \\
\hline Course F & Arts & 1 \\
\hline
\end{tabular}




\section{(4.5) Research Instruments and Procedures}

The research was undertaken in a series of three Phases, exploring the elements of the three central Research Questions. The data collection and analysis procedures are therefore presented in order of the three phases:

(4.5.1) Data Collection Methods for Text Profiling

(4.5.2) Data Collection Methods for Task Profiling

(4.5.3) Data Collection Methods for Reader Profiling

A summary overview of the data collection procedures and the participants and courses involved can be found in Appendix 1.

\section{(4.5.1) Data Collection Methods for Text Profiling}

In keeping with Dunworth's (2008, p.316) contention that the "local inspection of primary sources ... the material that students themselves encounter" is imperative, this study observed the interaction of 'real' students undertaking 'actual' university reading tasks with 'authentic' texts. To gain an understanding of the nature of authentic academic texts assigned to first year students as required reading, I undertook to profile the salient linguistic characteristics of these texts in the case study Courses.

The selected text samples for Courses A, B and C are listed in Appendix 2. For each first year Course chosen, the assigned readings/textbooks were collected and text samples between 200-400 words in length were selected for analysis. This length of Passage allowed participants to complete its reading in under 5 minutes and was the length found to be a vehicle for sufficient development of content so as to make the passages conceptually 'self contained'. It was also the length that allowed expediency of analysis for idea 
units. The following methods were involved in the profiling of the selected text samples.

Profiling the Lexical Composition of Authentic Academic Texts: Estimating the vocabulary challenge of first year academic Texts

In order to gain an understanding of the lexical composition and potential complexity of the texts, each text sample was analysed for lexical frequency. Each text sample was first typed as a Word file including all headings, words, text accompanying tables and figures. This text was then lexically profiled for word frequency using the online vocabulary profiling software called "VocabProfile" (Cobb, n.d.), which is based on Heatley and Nation's (1994) Range vocabulary profiling programme. The output from this analysis was a breakdown of the vocabulary of the text according to whether it came from the first and second 1000 word Levels, the Academic Word List (Coxhead, 2000), or whether the item did not occur on either any of the vocabulary Levels or the Academic Word List, in which case they are classified as 'Off-list' words. The number of word families, word types, tokens and other similar information was also obtained.

This text analysis enabled a number of useful lines of investigation:

- Generation of an understanding of the general lexical make-up of first year academic texts in general.

- A comparison between the lexical characteristics of texts from different disciplines.

- Comparison of text vocabulary profile results and results of Reader vocabulary profiling in light of previous research-based estimates of lexical thresholds. 
- Systematic observation of the relationship between the lexical profiles of texts and the Reader's responses to texts and their modes of interaction with them.

(4.5.1.2) Profiling the Syntactic Complexity of Authentic Academic Texts: Estimates of SC Based on Sentence Length

To gain an estimate of the Syntactic Complexity (SC) of the authentic first year texts the average sentence length (average number of words per sentence) was calculated using the following equation:

Average Sentence Length $=\frac{\# \text { wordsS1 } 1+\# \text { wordsS2 }+\# \text { wordsS } 3+\ldots}{\text { number of sentences in text sample }}$

where the Average Sentence Length is equal to the average number of words per sentence in the text sample, and \#wordsS1 represents the number of words in sentence 1 (S1), \#wordsS2 represents the number of words in sentence 2 (S2), and so forth, for as many sentences as there are in the text sample. This calculation was undertaken for the text samples used for the Free Recall protocol in Courses A, B and C.

(4.5.1.3) Profiling the Conceptual Density of Authentic Academic Texts: Calculations of the Idea Unit Density (IU Density)

To establish the Idea Unit Density (IU Density) of the first year authentic academic texts, a calculation of the Idea Unit Density was carried out based on the relative ratio of Idea Units (IU) to sentences (s) in the text samples. This ratio is taken to demonstrate the number of ideas a student reader must 'process' while reading each sentence. 
The analysis of the IU in this study does not pertain to the relationships between ideas in text or the function of these ideas within the text (such as has been explored by Meyer, 1975, Fredericksen, 1975; Spencer, 1973 (cited in Meyer, 1975)). In fact it does not pertain to the organization of information in prose at all, but rather to the systematic identification of the semantically based units within text. Meyer (1975, p.57) states that "it may be that one prose analysis procedure is superior for a particular research problem and inferior for others". In my survey of the literature I was unable to find an approach to prose analysis that would be suited to my particular needs in the research context in this study, hence the 'do-it-yourself', research-informed approach to the design of Idea Units and text analysis procedures.

My approach to prose analysis for IU in this project has two main functions. The first is for the purpose of calculating the Idea Unit Density (IU Density) of the text samples, as an indicator of the complexity and readability of the texts. The second is akin to that of Spencer (1973, cited in Meyer, 1975, p.71), whose system of prose analysis in her research dissertation was designed with the explicit purpose in mind of "objectify[ing] the procedures for scoring subjects' recall protocols".

The IU in this research is a unit that lies in between van Dijk and Kintsch's (1983) semantic building block, the 'proposition', and the older models proposed by the likes of Meyer and Spencer. In this study, an Idea Unit is understood to be a unit of meaning complete in and of itself, but which is linked to the IU around it by means of grammatical and semantic cohesive devices. The focus is on (i) the density of IU within sentences, and later, (ii) the existence of these IU within the Free Recall (FR) transcripts of these Pacific Reader participants who have undertaken the FR protocol. 
A criticism that has been aimed at many of the earlier attempts at measuring conceptual density, and of which I am very much aware, relates to the measures used being deemed "arbitrary and theoretically unfounded" (Kintsch \& Keenan, 1973, p.274). I nevertheless attest to its value as a system of classification of semantic content in text and as a means of understanding readers' processes by analyzing the recall of participants. In spite of these units being fairly describable, a certain degree of researcher subjectivity is unavoidable in this IU division process. While care has been taken to ensure that all the text is covered in a consistent and systematic manner during my analysis, this subjectivity may in future studies be able to be counterbalanced by the involvement of inter-raters.

Overall, in the formation of Idea Units the following guidelines are adhered to:

- An IU centers around one main verb only.

- An IU contains any additional descriptive elements only if these do not contain verbs (in this case they are usually considered to be Idea Units in and of themselves).

- IU must be able to stand alone (any ambiguous pronouns or elipted verbs are replaced with their full forms).

- Clauses linked by conjunctions are separated into separate IU.

- For subjects, objects, adverbs, adjectives and such like which are composed of entire clauses, decisions are made based on sense and meaning as to whether they are treated as separate IU entities.

Using these guidelines, every sentence in all the text samples was analysed, yielding a list of IU for that text sample. This was undertaken for the text samples used for the Free Recall protocol in Courses A, B and C. The total number of IU in the Passage was then divided by the total number of sentences to ascertain a ratio measure which I call Idea Unit Density. 
IU Density $=\frac{\text { total \# IU in Passage }}{\text { number of sentences in text sample }}$

The following process was used for the text sample analysis:

(i) Division of the text sample into sentences and establishing sentences length.

The text sample was divided into sentences. These were catalogued in a table such as the following for each text sample.

Table 4.3: Sample extract from text analysis table: Dividing text into sentences

\begin{tabular}{|l|l|l|}
\hline $\begin{array}{l}\text { Sentence } \\
\text { number } \\
(\#)\end{array}$ & $\begin{array}{l}\text { Sentence } \\
\text { length } \\
\text { (no. of } \\
\text { words) }\end{array}$ & Sentence \\
\hline s1 & 8 & The nature of the task is always important. \\
\hline s2 & 24 & $\begin{array}{l}\text { It affects how well a team can focus its efforts and how } \\
\text { intense the group process needs to be to get the job done. }\end{array}$ \\
\hline &
\end{tabular}

(ii) Analysis of each of the sentences in the text sample for IU.

Identification of the IU existing within sentences was undertaken (using the guidelines presented previously), yielding results that were presented in a table such as the one below:

Table 4.4: Sample extract from text analysis: Analysis of the sentences into IU

\begin{tabular}{|l|l|l|}
\hline $\begin{array}{l}\text { Idea } \\
\text { Unit } \\
\text { number } \\
(\#)\end{array}$ & $\begin{array}{l}\text { Sentence } \\
\text { ( })\end{array}$ & Idea Units \\
\hline 1 & $\mathrm{~s} 1$ & The nature of the task is always important \\
\hline
\end{tabular}




\begin{tabular}{|l|l|l|}
\hline 2 & s2 & $\begin{array}{l}\text { The nature of the task affects how well a team can focus its } \\
\text { efforts }\end{array}$ \\
\hline 3 & s2 & $\begin{array}{l}\text { The nature of the task affects how intense the group process } \\
\text { needs to be to get the job done }\end{array}$ \\
\hline & & $\ldots$
\end{tabular}

(iii) Calculation of IU Density and Syntactic Complexity

IU Density and SC were calculated as previously described. A final results table such as the one shown below was created for each text sample, containing key text sample profile information.

Table 4.5: Sample text profile summary table

\begin{tabular}{|l|l|}
\hline Text sample topic & Passage A9: Group Inputs \\
\hline Source & Campling, J., Poole, D., Retha, W., Ang, S.E., \\
& $\begin{array}{l}\text { Chan, B., Tan, W.L., Schermerhorn, J.R. } \\
\text { (2004). Management. 3 } \\
\text { Edition. Asia-Pacific } \\
\text { Ltd. }\end{array}$ \\
\hline Total Word count & 329 \\
\hline \# of Sentences analysed in text sample & 17 \\
\hline \# of Idea Units & 35 \\
\hline $\begin{array}{l}\text { Idea Unit Density (Ratio of sentences } \\
\text { to Idea Units) }\end{array}$ & 2.05 \\
\hline $\begin{array}{l}\text { Average Sentence Length (\# of words } \\
\text { per sentence) }\end{array}$ & 19.8 words per sentence \\
\hline
\end{tabular}

The resulting data tables for each sample Passage recorded key textual profile information in preparation for further data analysis.

Of the case study courses represented in the study, detailed text analysis was carried out for samples from Courses A B and C only, and while passages A1A11, B1-B9, C1-C5 were analysed for Vocabulary profile, only Passages A6A11, B5-B9, C1-C5 were analysed for Syntactic Complexity and Idea Unit Density. The reason for this is that only those passages involved in the Free Recall protocols were analysed for SC and IU Density. This was primarily 155 
due to constraints on the time available for the text analysis phase of the research and the primary need for detailed IU analysis of the Free Recall passages.

\section{(4.5.2) Data Collection Methods for Task Profiling}

The following methods were used to profile the academic reading tasks required of first year readers in the case study Courses A, B and C, the task representations of the students and staff, and their beliefs about what constitutes being a successful reader in this context. Of the six first year courses which the participants represented, Courses A, B and C have been chosen for observation since they represent three distinct discipline areas, and for certain aspects of my analysis Course A takes a more prominent place because of the fact that six of the participants were enrolled in that particular first year course.

A three-part approach was taken to data collection, comprising document analysis, participant interviews and classroom observations and from each of these the core academic reading tasks for the Course were deduced. These methods are outlined below.

\section{(4.5.2.1) Document Analysis of Course Outlines}

In order to gain an understanding of the authentic reading tasks required of first year students, document analysis was carried out on the Course Outlines of the case study Courses. Course Outlines are the documents given to students at the beginning of the trimester containing an outline of the Course content, administrative details and commonly a breakdown of the assessment required of the student in the Course. From these Course Outlines (CO) the core reading tasks/processes and outcomes required of students in each first 
year case study Course were identified and documented using the following steps:

(i) Document analysis

All references to reading, both explicit and implicit were identified in the $\mathrm{CO}$, extracted and recorded in a table. From each reading-related statement in the $\mathrm{CO}$ a statement beginning with "Read to..." was constructed, to indicate the reason for the reading required and the means by which it was expected to be carried out. The following is an example of the charts that were created for each of Courses A, B and C:

Table 4.6: Sample extract from task analysis: Course outline analysis for reading tasks

COURSE B - Analysis of Course Outline 2008 for reading tasks/processes

\begin{tabular}{|c|c|c|c|}
\hline $\begin{array}{l}\text { Course Outline } \\
\text { Excerpt }\end{array}$ & $\begin{array}{l}\text { Explanatory } \\
\text { notes }\end{array}$ & $\begin{array}{l}\text { Explicit reading } \\
\text { requirements }\end{array}$ & $\begin{array}{l}\text { Implicit reading } \\
\text { requirements }\end{array}$ \\
\hline $\begin{array}{l}\text { "This course } \\
\text { focuses on the } \\
\text { interplay of [topic] } \\
\text { and [topic]. } \\
\text { Diverse case } \\
\text { studies show how } \\
\text { [topic] and [topic] } \\
\text { intersect to create, } \\
\text { sustain and disrupt } \\
\text { established [topic] } \\
\text { systems." }\end{array}$ & & $\begin{array}{l}\text { Read a narrative on a } \\
\text { topic to analyse its } \\
\text { structure. } \\
\text { Read a narrative on a } \\
\text { topic to analyse its } \\
\text { context. }\end{array}$ & $\begin{array}{l}\text { Read to understand the } \\
\text { interplay of topics in } \\
\text { the discipline. } \\
\text { Read case studies to } \\
\text { understand how topics } \\
\text { intersect and affect } \\
\text { each other. }\end{array}$ \\
\hline$\ldots$ & $\cdots$ & $\cdots$ & \\
\hline
\end{tabular}

(ii) Participant checking

The explicit and implicit reading purpose statements were then checked by the participating Lecturers/Tutors. Minor alterations were made as necessary. 
(iii) Thematic analysis \& Identification of core generic and core specific Reading Tasks / Processes

From the "Read to..." statements a thematic analysis was conducted, resulting in a condensed list of the core reading purposes/tasks/outcomes for each Course. These lists were then coded and compared. The reading tasks common to two or more Courses were extracted and labeled as Core Reading Processes. The following is an extract:

Table 4.7: Sample extract from task analysis: Thematic analysis of shared reading tasks

Core Reading Tasks/Processes Overlapping Two or More First Year Case Study Courses

\begin{tabular}{|l|l|l|}
\hline $\begin{array}{l}\text { Found } \\
\text { in }\end{array}$ & Shared Reading Task/Process & Shared theme \\
\hline A & $\begin{array}{l}\text { Read to critically understand key material/ideas } \\
\text { in order to facilitate a tutorial session and } \\
\text { generate original ideas to contribute to the } \\
\text { tutorial group/discussion. } \\
\text { Read to understand the concepts/topics within } \\
\text { reading in order to be able to participate in } \\
\text { tutorials. } \\
\text { Read to identify/understand key points/concepts } \\
\text { in set readings in order to be able to facilitate } \\
\text { and generate discussion with peers in tutorials. }\end{array}$ \\
C & $\ldots$
\end{tabular}

The remaining "Read to...' statements in each Course were labeled as the core Reading Tasks/Processes specific to each case study Course. Each core reading task was labeled for coding and analysis of the other data sources. The following is an extract: 
Table 4.8: Sample extract from task analysis: Documenting course-specific reading tasks

Course-Specific Reading Processes for Course A

\begin{tabular}{|l|l|}
\hline Label & Reading task/process specific to Course A \\
\hline A(a) & $\begin{array}{l}\text { Read to understand/critically analyse theories/concepts in order to be able to } \\
\text { recognize and interpret and examine instances of their application in real } \\
\text { life situations/events/behaviours. }\end{array}$ \\
\hline A(b) & $\begin{array}{l}\text { Read to form a basic understanding to then have this built upon/extended (in } \\
\text { lectures and further learning). }\end{array}$ \\
\hline$\ldots$ & $\ldots$
\end{tabular}

(iv) Triangulation of data sources

Each core and Course-specific reading task was coded and compared to the information gathered from (a) observation of classes and (b) staff and student interviews. Those tasks found to be supported by the data from the other data collection methods were confirmed as the core reading processes for the Course.

\section{(4.5.2.2) Interviews with Lecturers/Tutors}

Semi-structured interviews were conducted with the Lecturer/Tutor/Course coordinator of the case study Courses. Discussion with the Course Coordinator/Lecturer/Tutor was based on the Course Outline and clarification, extension and description was sought for various parts of the $\mathrm{CO}$, the instructor's intentions for the reading practices, processes and outcomes, and the Lecturer's /Tutor's conceptions of the reading tasks and ways of reading in their Course and in the discipline as a whole. Lecturers' interviews provided information concerning text selection, pedagogical position, philosophical position, reason for reading, way to read, understanding of students, and 
thoughts on the Course Outline analysis. Interview notes were summarized and themes were drawn from them.

\section{(4.5.2.3) Classroom Observation}

With the Lecturer/Tutor's permission I attended one or more 'typical' classes/tutorials of the staff member's choice. Field notes were taken as unobtrusively as possible, documenting the situations and contexts of reading or reading-related activity in the classrooms, evidence of students having done or not done readings, and both the Lecturer/Tutor's and the students' use of content from the readings in the classroom. Notes were summarized and themes were drawn from them.

Having identified the most common core Tasks I was able to choose the most basic ('reading to understand and remember'). By holding this factor of the Text-Task-Reader interplay stable, it was possible to observe the interaction of the other two - the Readers and the Texts.

\section{(4.5.3) Data Collection Methods for Reader Profiling}

In profiling the Readers, a composite skills approach was taken and the following holistic range of contributing factors was investigated.

\section{(4.5.3.1) Gaining an Understanding of Pacific Readers' Reading Histories: Reading History Interviews \\ For the purpose of gaining an understanding of the Pacific case study participants' reading histories I interviewed them on their most salient reading- related memories from various stages of their home and educational histories. During the interview, which lasted on average between 25-40 minutes, I asked the students to recall their most salient memories of the following things during their childhood and adolescent years:}


- languages spoken at home and in what amounts

- country of birth and education of both themselves and their parents/caregivers

- the presence of books and bookshelves in their home, and the role of these

- the reading habits of family members

- the reading habits of their closest friends

- $\quad$ attendance at pre-school, and any early reading experiences

- $\quad$ reading habits at school between the ages of 5-7 years (in-school reading)

- any reading done for non-school-related purposes (out-of-school reading)

- in-school reading and out-of-school reading between the ages of 8-10 years

- in-school and out-of-school reading in early high school years (up to Form 3 equivalent)

- in-school and out-of-school reading occurring between $3^{\text {rd }}$ Form and $5^{\text {th }}$ Form

- $\quad$ in-school and out-of-school reading experiences from $5^{\text {th }}$ to $7^{\text {th }}$ Form in high school

- their own feelings about themselves as readers and their development as readers.

Due to the personal nature of the content of the interview, the informal manner of the interviews, and the early stage of the researcher-participant relationship, I felt strongly that it was culturally inappropriate to use a recording device. Thus, while listening to them I took written notes. My background as a learning advisor has equipped me well with skills for listening and note-taking in an accurate, comprehensive and largely unobtrusive way. It was not until I had developed a trusting relationship with the student participants over more than half a trimester that I used the recording device, and this was only used for the recall protocol (where participants recalled passage content), not for interviews in which personal reading issues and weaknesses may have been revealed and discussed.

I believe strongly that part of the relative success of the study in engaging and sustaining voluntary participation in the study by the Pacific students was due to my awareness of and care to uphold the 'safety' of my participants by 
minimizing the engagement of potentially face-threatening and powerdistancing data collecting procedures (such as the immediate use of recording devices) in the early stages of the project while the crucial trust-building stages of the relationship underway. This I believe also contributed to the openness of the participants in their discussions with me, as well as their willingness to see the study through to completion over more than a trimester in length.

These notes made during semi-structured interviews/discussions with the participants were then compiled in tabular form for each Reader and used as reference for reader profiling and for understanding whether/how certain trends in reading histories could be traced to the existence of features in their various reading profiles. The following is an extract of these tables to illustrate:

Table 4.9: Sample extract from reader profiling: Reader's reading history interview summary notes

S13(PF) - Pacific born female

\begin{tabular}{|c|c|}
\hline First language: & Samoan. \\
\hline Parents: & Parents born in Samoa. \\
\hline Siblings: & Has siblings and they converse in Samoan. \\
\hline $\begin{array}{l}\text { Home } \\
\text { languages } \\
\text { spoken: }\end{array}$ & $\begin{array}{l}\text { Her parents had gone to another Pacific nation as missionaries } \\
\text { and three children were born there. She received all her } \\
\text { primary and secondary education in Samoa, she spoke } 100 \% \\
\text { Samoan language in the home. }\end{array}$ \\
\hline $\begin{array}{l}\text { Pre-school } \\
\text { reading } \\
\text { memories: }\end{array}$ & None \\
\hline $\begin{array}{l}5-7 \text { yrs reading } \\
\text { memories: }\end{array}$ & $\begin{array}{l}\text { At her local primary school in Samoa they used both Samoan } \\
\text { and English languages. She recalls learning the alphabet in } \\
\text { English. ... }\end{array}$ \\
\hline
\end{tabular}


My decision not to voice record my participants in these interviews means that there is seldom verbatim evidence to provide, but through systematic comparison, themes were drawn from the similarities and differences and repeated ideas present in the tables.

\section{(4.5.3.2) Gaining an Estimate of Receptive Vocabulary Size: Vocabulary Size Test}

In order to gain an estimate of the size of a student's receptive vocabulary the Vocabulary Size Test designed by Beglar and Nation (2007) was used. It was originally designed to "see how close the learner is to having enough vocabulary to be able to perform certain language use tasks" (Nation \& Beglar, 2007, p.1).

Each participating Pacific student Reader was given the vocabulary measure for the 14 vocabulary levels. In the measure, 10 items from each of the 14 levels have been selected and each is presented in a short sentence. The student must then choose the correct definition from a choice of four possible definitions. Having decided on the correct meaning, they circle the letter corresponding to that definition. Due to the constraints of the thesis this vocabulary measure itself is not included but can be easily retrieved in print form from Cobb's Frequency Based Vocabulary Tests website (Nation, 2007).

For this study a cover sheet was provided, explaining the source of the test and giving an example of how to answer one item. The instructions were also delivered verbally and when it was ascertained that they understood the task they began. The measure took on average between 30 to 40 minutes to complete. Each student completed the measure on their own and without the use of dictionaries or any other reference material. The majority of the Readers conducted the test in my presence, but a few took it away to complete. 
Once the student had completed the measure it was collected and marked. A score out of ten for each of the fourteen levels was generated, and these (when multiplied by 10) were taken as representative of an estimated percentage knowledge of that vocabulary level. The other university students who also undertook the 14 levels vocabulary size test were emailed the test and a simple answer sheet into which they were asked to type the letter of the answer they chose for each item.

The Pacific student participants also undertook the Academic Vocabulary test containing 30 items drawn from the corpus based University Word List. The measure was administered similarly to the Vocabulary Size Test and the score out of 30 achieved was converted to a percentage out of 100 as an indication of their estimated knowledge of the UWL items.

These results facilitated the following analyses:

- The estimated vocabulary size results were then plotted on a graph to observe patterns both group (combined averages) and individual patterning. A comparative analysis was carried out between the vocabulary results of Pacific and non-Pacific participants using a statistical t-test to observe whether differences existed between average scores of the two groups of readers.

- Observations of patterning across the 14 levels (for the Vocabulary Size Text).

- Calculations to estimate the likely ability of the first year Pacific student participants to be able to independently and successfully comprehend a particular authentic text within their first year, based on $\mathrm{Hu}$ and Nation (2000) and various others' research on vocabulary thresholds.

- Calculating estimates of percentage Text Coverage provided by a combination of several core vocabulary sets; K1, K2 and the AWL. 
- Comparison of these Text Coverage estimates against success on task (represented by \% IU Recalled in the Free Recall Task) to observe any relationship between vocabulary knowledge on task performance.

- Comparison of the vocabulary profile of Texts against Readers' affective responses to text, in order to gain an indication of whether Text vocabulary influences Readers' affective responses to Text.

If no findings of interest or significance were found, the measures have not been presented for discussion in this thesis, but all were undertaken as lines of investigation.

\section{Measuring Reading Rate: Timed Reading of Authentic Academic Text Samples while Undertaking the Task of 'Reading to Understand and Remember'.}

To gain an estimate of the Rate at which the participating Pacific students read in order to 'understand and remember' authentic text within their discipline, they were timed as they carried out the task with the authentic academic text samples from their Course. Readings chosen were those not yet covered in class as set reading. In terms of text sample selection, this was done manually according to the following criteria. The authentic text samples selected were:

- from the actual texts assigned by the course coordinator as the set and required reading for that course.

- from a text that the student could be expected not to have yet read at that point in the trimester.

- between 250-400 words long.

- taken from a number and variety of those sources when more than one existed.

- relatively 'self contained' and dealing with a 'complete' idea or set of ideas, such that in order to understand them it was not necessary to have read the preceding paragraphs/sections. 
The pages containing these selected text samples were colour photocopied, and to ensure authenticity, care was taken to preserve the characteristics of the text as the student would naturally encounter it in its source (textbook or book of readings). Each student participant undertook the task with their own set of these text samples to enable them to write on the text and interact with it in a natural manner. In all cases it was explained to the student where the text sample had been taken from.

To gain Reading Rates data indicating the speed at which these Passages were read for understanding and remembering purposes, the participants were instructed that they should indicate to me when they began and finished reading. The time taken was established and recorded in seconds using a wristwatch with a second hand and clear numbering. Using the following formula, the Reading Rate in words per minute was calculated:

Reading Rate $=\frac{\text { Number of words in passage }}{\text { Time taken to read it (in seconds) }} \times \frac{60}{1}$

This calculation yielded a score in 'words per minute' (wpm), and was done for each student's reading of each of the selected text samples from that Course. The use of 'Rate' rather than 'speed' accounts for the fact that it is the time taken to complete the task, not merely to decode the Passage (i.e. including time for rereading, pausing, highlighting, or whatever other strategies the reader used to complete the task).

Having established the Rates at which the various Readers read the text, various other cross tabulations were possible to observe whether relationships of any systematic nature existed between Reading Rate and any features of the texts themselves. This was of particular interest because of the links that have 
been suggested by previous researchers between the properties of text and reading speed (Kintsch \& Keenan, 1973, p.258; Perfetti, 1969). The possible existence of relationships between Rate and the following characteristics was investigated:

- Estimated Text Coverage by K1+K2+AWL

- Specific Features of the Text Vocabulary Profile

- Idea Unit Density

- Syntactic Complexity (average sentence length)

- Text sample length.

Also of interest were any relationships between Reading Rates and Readers' characteristics. Rate was plotted against various Reader variables including Estimated Vocabulary Size, Reading Strategy use and Attitudes, and success in prose retrieval (\% IU Recall). Plotted data in graph form allowed for visual observation of trends. All other comparisons in the study also took this form because the samples sizes were too small to warrant fuller statistical processing.

Measuring and Describing the Characteristics of Idea Unit Recall through (i) Immediate Written Prompted Recall and (ii)Immediate Spoken Free Recall

The task chosen for this study was 'reading to understand and remember' and in investigation of text comprehension and recall in a learning context it is important to note that there is a distinction between 'reading to learn' and 'reading to remember', and that one does not necessarily entail the other. Kintsch (1998, cited in Koda 2005, p.138) contends that "remembering entails the ability to reproduce the text verbatim or through summarization, whereas learning requires the capacity to use freshly acquired information productively in a new context". In order to gain an understanding of the amount of content 
(in terms of the Idea Units in the Passage) that participant Readers could recall after one reading (for the purpose of reading to understand and to remember), two measures were used - Prompted Recall, and Free Recall. Prior to the use of either protocol however, the participants had the procedure explained to them and friendly conversation was carried out to put participants at ease and ascertain their understanding of the process and its purpose, and their willingness to have their spoken recollections recorded.

Central to both the Recall measures was the concept of the Idea Unit described previously in this chapter. The first measure was the immediate written Prompted Recall, whereby Readers were given a set of simple factual recall questions generated from the main Idea Units in the text. Approximately one question per main idea was produced, with the aim of ascertaining how many of the central idea units were being recalled by each student with each of the selected text samples. The prompted recall was conducted for the two main case study Courses but abandoned part way through data collection, after it was found to be impractical. For the main case study Courses the second protocol was used.

The second protocol was the immediate spoken Free Recall (FR). This is strongly supported by Lee (2009, p.200) who concluded that "immediate postreading free recall tasks are preferable to multiple-choice questions" for assessment of reader comprehension, and they are very valuable for:

- 'tracking a reader's psychological processes while minimizing researcher bias" (p.200).

- providing rich evidence for understanding a reader-based constructivist modeling of the reader's processes (p.20) 
- providing an indication of comprehension that is "uncomplicated by linguistic performance and tester interference" (Bernhardt, 1991, cited in Lee, 2009, p.200).

- offering an "enhanced understanding of the qualitative features of L2 readers' comprehension and how they may be affected by cognitive and affective variables like topic congruence and topic interest" (p.163).

Free Recall as a procedure and the concept of Idea Units has also been used extensively and effectively by Armbruster and Anderson (1980) and various other associates throughout the 1980s and early 1990s, for the purposes of exploring the effects of text idea organization and idea mapping on student readers' text comprehension.

In the Free Recall protocol participants were asked to verbally recall what the Passage was about immediately (within one minute) after reading it. After the participant had indicated that they had finished reading and the time had been recorded, the Passage was turned face downwards and participants were asked "What was that passage about?" The participants then proceeded to verbally recall whatever they had understood and could remember from the text. Their spoken Recall was recorded on a small digital voice recorder whilst I simultaneously took written notes. The presence of the digital recorder was suitable in this context due to the formalized nature of the interaction between myself and the participants during the FR protocol and the rapport and trust that had by this point been established. The digital recordings were then transferred to a computer and digital voice recording software was used to enable me to transcribe each participant's spoken recollections. The Readers' transcripts for Course A can be found in Appendix 3. 
These transcriptions were then 'cleaned' to remove fillers, repetition, hedging and unrelated comments, and the 'cleaned version' was scored for presence of Idea Units from the original text. Analysis of the transcripts and their post task interviews allowed a better understanding of the nature of the Readers' Recall and the processes by which they undertook the task of 'reading to understand and remember'.

It was then that connections were able to be made between the findings of the Text profiling and the Reader profiling. The integration of data sets throughout the study was designed to shed light on any possible connections between the characteristics of the Texts in question, the ways in which Readers responded to them, the ways they made meaning from them, and the degree of success they had in reading them for the purpose of understanding and remembering.

(4.5.3.5) Gaining an Understanding of Readers' Reported Affective and Perceptive Responses to Text: Reader Ratings of Text Difficulty and Text Interest

In order to investigate the nature and the impact of Readers' affective responses to Text, each case study Reader was asked to rate the Passage just read in terms of perceived Difficulty and Interest and their perception of the Difficulty of the Vocabulary. Although this data was collected from most participants, analysis focused on Course A, in which six case study Readers responded to each of the 12 Passages read (with a few exceptions). This then presented a sizeable amount of data to work with. The ratings yielded insights into perceived Passage Difficulty and Interest, as well as Readers' characteristic responses to text and how this related to other variables both within the Text and the Reader themselves. 
Readers' perceived ratings of Passage Interest and Difficulty were crosstabulated against:

- Text vocabulary profile features

- Idea Unit Density

- Syntactic Complexity estimates

- \% IU Recall in the FR task

With data plotted on charts it was possible to gauge, for this group of participants undertaking this particular task, the existence of relationships between the various Text and Reader features. The data averages also allowed insights into each Reader's overall affective reactions to the Texts in their Course. There is considerably more information that can be drawn from these results than has been reported on in this thesis due to the limitations of space. As such, only the most notable findings have been presented.

\section{(4.5.3.6) Documenting Readers' Reported Reading Strategy Use: Academic Reading Strategies Inventory}

A great deal of literature and research exists on this issue. It appears to be unanimous in its support of the importance and efficacy of academic reading strategy instruction, but significantly less unified on the issue of the nature and contents of the measures for such reading strategies. Thus inventory of academic reading strategies, habits and attitudes was constructed for this study through which Readers could self report on their own reading strategy behaviour and attitudes. The inventory was synthesized from a number of existing inventories and from research findings on 'good' and 'poor' readers (Chapter 2). This measure can be found in Appendix 4 and was piloted with several students and refined for the purposes of use in this research. Further refinement of the instrument after piloting, involved discarding of ambiguous items and shortening and clarification of wording with assistance from colleagues and students. 
The Academic Reading Strategies, Habits and Attitudes Inventory used in this study consists of 71 items, falling into nine categories:

Affective and Attitudinal

This category has been defined by grouping affect-related items, including items relating to:

- personal preference in reading material

- self-concept as a reader

- motivation for reading

Selective and Directed

This category has been defined by grouping strategies relating to:

- $\quad$ selective reading

- previewing

- recognition of text structure

- response to text flow

- $\quad$ linking of ideas within the text

Comprehension \& Comprehension monitoring Strategies

This category has been defined by grouping strategies relating to: comprehension monitoring strategies

- $\quad$ annotation on the text

- summary creation

- questioning

- elaboration

- inference

- simplification

- predicting

- paraphrasing

- visualizing

- discussion with others

- consulting reference material

- memorising 


\section{Typographical related strategies}

This category has been defined by the grouping of items relating to visual aspects of the text, including use of:

- graphics and pictures in text to aid understanding

- typographical features of text to aid understanding

- headings and subheadings to aid understanding

Evaluation type strategies

This category has been defined by grouping strategies relating to:

- judgments about the value and truth of content/ideas in text

- judgments about how the author has constructed the argument

- distinguishing lines and types of argument

- identifying inconsistencies in the text

- identifying and comparing attitudes/arguments within a text

Strategies affecting reading rate and rate regulation

This category has been defined by grouping strategies relating to:

- vocalizing and subvocalizing

- rate adjustment according to text and task

- pausing

- rereading

- word recognition speed

- span of phrases in reading

\section{Utilizing prior knowledge}

This category has been defined by grouping strategies relating to:

- linking of text ideas to prior world/general knowledge

- linking of text ideas to prior learning

Author awareness and intertextuality

This category has been defined by grouping strategies relating to:

- identifying author's main arguments

- consideration of the author's context

- intertextuality and the connection between various authors' ideas 


\section{$\underline{\text { Vocabulary related strategies }}$}

This category has been defined by grouping strategies relating to:

- guessing of unknown words

- looking up unknown words in reference material

- conscious acquisition of new vocabulary in a text

- translation of unknown vocabulary into the L1

- maintaining meaning focus when dealing with unknown vocabulary

Each item and category was selected from literature, and multiple items exist for the strategies considered in literature and in my own experience to be of greatest use or interest in the study of the group in this project. Each item is phrased as a statement in the first person. For example, the strategy of purpose setting prior to reading the statement in the inventory is phrased as:

"Before I read I make sure I am clear about my purpose for reading" Opportunity for clarification and explanation of the statements was available throughout the exercise and the students were encouraged to ask if they felt they had not understood the statement. Each student, having listened to the statement being read aloud to them, then looked at the 7 point scale provided (see Figure 4.1 below), considered their general approach to reading in this Course, and said aloud the number corresponding to the answer that suited them best for that item.

\begin{tabular}{|c|c|c|c|c|c|c|}
\hline 1 & 2 & 3 & 4 & 5 & 6 & 7 \\
\hline $\begin{array}{c}\text { NEVER } \\
\text { TRUE }\end{array}$ & $\begin{array}{c}\text { ALMOST } \\
\text { NEVER } \\
\text { OFUE } \\
\text { OF ME }\end{array}$ & $\begin{array}{c}\text { USUALLY } \\
\text { NOT } \\
\text { TRUE } \\
\text { OF ME }\end{array}$ & $\begin{array}{c}\text { AITTLE } \\
\text { BIT } \\
\text { TRUE } \\
\text { OF ME }\end{array}$ & $\begin{array}{c}\text { USUALLY } \\
\text { TRUE } \\
\text { OF ME }\end{array}$ & $\begin{array}{c}\text { ALMOST } \\
\text { ALWAYS } \\
\text { TRUE } \\
\text { OF ME }\end{array}$ & $\begin{array}{c}\text { ALWAYS } \\
\text { ORU ME }\end{array}$ \\
& & OF & & \\
\hline
\end{tabular}

Figure 4.1: Reading strategy self-reporting scale (adapted from Zhang, 1994) 
The self-reported 'scores' for the Inventory were recorded on a score sheet. The Inventory measure took, on average, 20 minutes to complete. The scores of all study participants yielded aggregate as well as individual patterns.

The Inventory items were categorized according to major themes in reading strategy research literature (Chapter 2) and overall student ratings in this study did appear to point to tolerable consistency within categories. Although this inventory was not designed following traditional survey construction methods, and could be further refined for future research, it has proven very useful as a means of profiling the particular strategic and attitudinal characteristics of these Pacific Readers.

(4.5.3.7) Documenting Readers' Overall Experiences of the Course and Beliefs about Academic Reading in First Year Courses: Final Interview

To gain an understanding of each case study Reader's beliefs about reading built up over the 12 weeks of the Course, an interview was conducted after the examination period with as many of the research participants of Course A (the case study Course) as were available at this time.

In this interview they were asked about their understandings of:

- The role of reading in the Course

- Perceptions of the set text

- Main uses of the set text

- Necessity of reading the set text

- $\quad$ Task clarity in the course \& Perceptions of Lecturer modelling reading

- Strategies/Skills believed to be useful for study

- Strategies for dealing with unknown vocabulary

- Personal beliefs about student reader's role

- Awareness of Author \& authority over text 
- Reading attitudes

- Perceived Effect of Culture and Background

- Lessons learnt about reading in this course

- Impact of being involved in the study

- Their own academic performance

Notes were taken during the interview, collated, and used to inform individual Reader profiles as well as aggregate understandings of student beliefs about reading in the Course and the discipline.

Recording Readers' Academic Achievement: Ascertaining Readers' Academic Results for the Course

To ascertain the ultimate academic performance of the Readers in the case study Course permission was sought in the final interview (discussed above) for Readers to report to me on either their actual final grade or, if they were reluctant to reveal this, to simply report whether they passed or failed the course. The majority of the participants told me whether they had passed or failed the Course.

\section{Chapter Summary}

In summary, the three-part design of the project enabled identification of the characteristics of the Texts, and the key Tasks, as well as the profiling of the Pacific Readers. Thus, the strands, and their means of preparation and weaving has been presented. The following chapters present and discuss the findings gained in relation to the central Research Questions, beginning with the findings of the profiling of the authentic academic texts. 


\section{CHAPTER 5}

\section{ACADEMIC TEXTS IN FIRST YEAR COURSES: FINDINGS \& DISCUSSION}

The findings and discussion in this chapter address Research Question 1(a): What is the nature of the typical academic texts encountered in the first year of university study?

(a) What are the key linguistic characteristics of typical authentic first year academic texts in Faculty $\mathrm{X}$ in terms of their

(i) lexical profile

(ii) grammatical complexity

(iii) conceptual density

In this chapter the findings of investigation into the salient characteristics of the authentic academic texts in the first year courses at the case study New Zealand University are presented and discussed. These characteristics were identified through analysis of samples of actual required reading from these Courses within the Humanities and Commerce faculties.

The chapter is presented in three parts, each describing different characteristics of Text chosen for the profiling of first year texts.

(5.1) Lexical complexity of authentic first year Texts (findings from lexical profiling of case study Course Texts)

(5.2) Grammatical complexity of first year Texts (findings from analysis of text samples)

(5.3) Conceptual density of first year Texts (findings from Idea Unit analysis of text samples) 
The findings of these sections provide an understanding of the nature of the Texts in the first year of study, and also provided the basis for investigation of the interaction between the Readers and the Texts discussed in subsequent chapters.

\section{(5.1) Lexical Complexity of Authentic First Year Texts: Vocabulary Profiles}

The results of lexical profiling of the text samples from several first year Courses in the Humanities and Commerce Faculties demonstrate wide variation both across and within disciplines. While participants in this study were enrolled in six courses, three Courses (A, B and C) have been chosen to illustrate the differences between disciplines because they all represent quite distinctly different academic disciplines. Later focus on Course A is because of the largest number of participants having been enrolled in this course ( 6 of the total of 19 participants who volunteered to be part of this study.

The lexical profiles of the text samples taken from Courses A, B and C are presented in Appendix 5. Comparison of these vocabulary profiles reveals a number of key differences in the nature of the Texts across the first year case study Courses. The most notable are presented below: 
Table 5.1: Comparison of sample text vocabulary profiles for Courses A, B and $\mathrm{C}$

\begin{tabular}{|l|l|l|l|}
\hline & Course A & Course B & Course C \\
\hline K1 & $71.16 \%$ & $73.1 \%$ & $72.32 \%$ \\
\hline K2 & $6.81 \%$ & $5.21 \%$ & $6.06 \%$ \\
\hline AWL & $12.98 \%$ & $5.72 \%$ & $11.45 \%$ \\
\hline Off-list words & $8.65 \%$ & $15.73 \%$ & $9.86 \%$ \\
\hline Number of word types & 181.6 & 152.4 & 182.8 \\
\hline \% of Function words & $40.17 \%$ & $45.4 \%$ & $40.57 \%$ \\
\hline \% of Content words & $30.99 \%$ & $27.6 \%$ & $31.75 \%$ \\
\hline Type-Token ratio & 0.52 & 0.55 & 0.53 \\
\hline Lexical density & 0.59 & 0.54 & 0.59 \\
\hline
\end{tabular}

The main differences between the Courses' Texts can be seen in the percentages of AWL and Off-List words, with Course A and C texts possessing more AWL but fewer Off-list words than Course B texts.

The disparity between the 5\% coverage of Course B texts by the AWL and the $11 \%$ and $12 \%$ coverage by AWL of the texts from Courses A and C is clear. This is a strong indication of the different nature of the texts in Course B, and tallies well with the Lecturer's comments on text choice in the discipline which revealed that these texts are often not traditionally 'academic' by nature. According to the Lecturer they may consist of first-hand accounts of the concepts/ideas being studied, sometimes taking the form of books of published field notes, or narratives in the first person, and such like. The text samples in Course $\mathrm{A}$, on the other hand, came from a textbook written by academics specifically for first year university students, and Course $C$ texts were drawn largely from scholarly books and journals traditionally viewed as more typically 'academic'. The differences in the sources and nature of first year set texts is clearly revealed in certain aspects of their vocabulary profiles, which 
has implications for the ways in which readers will experience and utilize these texts.

The existence of such variation in the nature of the core texts selected for first year courses is most probably a natural consequence of the specialization into disciplines, however it does raise questions about the comparability of the first year reading experience across the University. It is not by chance that a student may cope well with the readings in one paper but founder when trying to navigate their way through the texts of another discipline even within the same Faculty. Nor is it by chance that some Courses gain a reputation of being 'difficult' or 'easy'. This reputation seems to be due at least in part to the nature of the Texts selected as core to the Course, and to what is expected of readers.

In terms of Off-List words another clear difference between the discipline texts is seen. Courses A and C are comprised of 8.6\%-9.8\% of Off-list words, while on average $15.7 \%$ of Course B texts are Off-List items. This indicates a clearly higher amount of low-frequency, borrowed, technical and specialist lexis in Course B texts. The results of the impact of Off-List words on these Pacific Readers were also observed when data were analysed. There is evidence that Course B Texts present some unique and considerable challenges for these Pacific Readers. However, due to the small number of cases these findings are not discussed in any detail here.

A strong difference between the text samples also lay in the Number of Word Types found in the extracts, with text samples from A and C possessing on average, a higher number of Word Types per sample than Course B. Interestingly, although a smaller overall selection of different words were used 
in Course B, the Off-List percentages indicate that a majority of those used were in fact technical and subject specific words.

Slight differences were also seen in the percentages of the Text samples that were Function words and those that were Content words, with Passages from Course B scoring higher for Function words and lower for Content words than Course A or C text samples. Accordingly, the average Lexical Density of the Course B texts was lower than that of Courses A and C. The higher average percentage of Function words in Course B Texts may be due again to the nature of those texts being not typically 'academic'.

Thus, the lexical profiling has provided convincing evidence that first year texts used by different disciplines contain differing lexical profiles. This has immediate implications for the academic reading experience for students within these first year Courses and for discussions around the comparability of the first year academic reading literacy experience. These measures of specific vocabulary features can also be of some use in predicting the potential difficulty students may experience with the set texts based on indications of students' accuracy rates in recall and their affective responses to text.

It is important to note however that the different numbers of sample passages utilized in each of the Courses A, B and C works to limit the inferences that can be drawn from the comparison of vocabulary profile in this study.

\section{(5.1.1) The "Off-list" Words, their Nature and Significance}

Consideration of the profile data category called 'Off-List' words is also instructive. Across the three first year Courses that form the main focus of this study, the average coverage by 'Off-List' words was $8.65 \%, 15.73 \%$ and 9.86\% for Courses A, B and C respectively, producing an overall indication 
that $11.4 \%$ of the texts fell into this Off-list category. This is of interest because it essentially means that over a tenth of the words in the text samples came from sources other than the K1-K14 lists and the AWL. The potential sources of this vocabulary include other low frequency English words, specialist, technical and subject-specific vocabulary items. Manual analysis of these items showed that the Off-list category contained:

- Proper Nouns

- Academic-type words not in the AWL

- Specialist technical vocabulary.

It must be noted, however, that the estimate does not necessarily entail $11 \%$ of brand new items, since repetition of "Off-list" items in the text played a noticeable role in bulking up the Off-list percentage. The situation may therefore not be as difficult as it seems. If, for instance, an Off-list item was a proper noun (place or person's name) and this was repeated with frequency in the text it could come to represent in figures what may appear to be a hurdle for the reader, but which in reality may not be. This is assuming of course that the reader possesses the ability to recognize the word as a proper noun and to recognize it each time it occurs without taxing the cognitive processes involved in comprehension. However, even if accommodation was made for repetition, and the existence of recognisable words such as proper nouns, the percentage of Off-list words left is still likely to comprise a substantial enough proportion of the text to cause some effect on comprehension and particularly, as this study found, on reader attitudes to and impressions of the text. 


\section{(5.2) Grammatical Complexity of Authentic First Year Texts: Estimates of Syntactic Complexity (SC) Based on Sentence Length}

As indicated previously, the measure of sentence length (in words) as indicative of the syntactic complexity of the text has been employed in this study. The following results were obtained from analysis of the Text samples (of only those texts involved in Free Recall) taken from set texts in Courses A, $\mathrm{B}$ and $\mathrm{C}$ :

Table 5.2: Syntactic complexity of passages in Courses A, B and C

\begin{tabular}{|c|c|}
\hline Passage & $\begin{array}{l}\text { Syntactic complexity } \\
\text { (Average number of words per sentence) }\end{array}$ \\
\hline \multicolumn{2}{|c|}{ Course A } \\
\hline A6 & 14.7 \\
\hline A7 & 20.85 \\
\hline A8 & 21.62 \\
\hline A9 & 19.8 \\
\hline A10 & 19.58 \\
\hline \multirow[t]{2}{*}{ A11 } & 15.38 \\
\hline & Average: 19 words per sentence \\
\hline \multicolumn{2}{|l|}{ Course B } \\
\hline B5 & 29.05 \\
\hline B6 & 19.9 \\
\hline B7 & 28.6 \\
\hline B8 & 19.3 \\
\hline \multirow[t]{2}{*}{ B9 } & 32.7 \\
\hline & Average: 26 words per sentence \\
\hline \multicolumn{2}{|l|}{ Course $\mathrm{C}$} \\
\hline $\mathrm{C} 1$ & 42.0 \\
\hline $\mathrm{C} 2$ & 33.5 \\
\hline $\mathrm{C} 3$ & 23.4 \\
\hline $\mathrm{C} 4$ & 35.1 \\
\hline \multirow[t]{2}{*}{$\mathrm{C} 5$} & 33.07 \\
\hline & Average: 33 words per sentence \\
\hline
\end{tabular}


Without having measured any other types of text it is not possible to comment on whether these texts represent comparative difficulty or ease in terms of their Syntactic Complexity. Nevertheless, it can be fairly stated that an average sentence length of 33 words per sentence (with maximum sentence lengths frequently reaching 40-56 words per sentence) does imply a certain level of complexity.

These results also indicate, once again, the variation between disciplines and text types/genres. Clearly the authentic scholarly and academic Texts in Course $\mathrm{C}$ are of greater Syntactic Complexity than those in the first year textbook in Course A, and the 'not typically academic' texts of Course B (which fall in between in terms of grammatical complexity). The implication is that Course $\mathrm{C}$ texts would pose a considerable challenge for the kinds of readers for whom grammatical complexity proves a hindrance to their text comprehension (for instance ESL or EFL readers).

\section{(5.3) Conceptual Density of Authentic First Year Texts: Idea Unit (IU) Density Measures}

The Idea Units within the sample Passages from Courses A, B and C were identified, and are presented in Appendix 9. The following results were obtained from analysis of the conceptual density of text samples from Courses A, B and C that were used for Free Recall. 
Table 5.3: Idea unit density of the passages in Courses A, B and C

COURSE A

\begin{tabular}{|l|l|l|l|l|l|l|l|l|l|l|l|}
\hline & \multicolumn{9}{|l|}{ Text Samples } \\
\hline & A1 & A2 & A3 & A4 & A5 & A6 & A7 & A8 & A9 & A10 & A11 \\
\hline $\begin{array}{l}\text { Idea Unit } \\
\begin{array}{l}\text { Density } \\
\text { (Average } \\
\text { number of IU } \\
\text { per sentence) }\end{array}\end{array}$ & - & - & - & - & 2.15 & 2.28 & 2.68 & 2.05 & 2.17 & 1.88 \\
\hline
\end{tabular}

Average IU Density for Course A Texts: 2.2

COURSE B

\begin{tabular}{|l|l|l|l|l|l|l|l|l|l|}
\hline & \multicolumn{6}{|l|}{ Text Samples } \\
\hline & B1 & B2 & B3 & B4 & B5 & B6 & B7 & B8 & B9 \\
\hline $\begin{array}{l}\text { Idea Unit Density } \\
\text { (Average number of IU per } \\
\text { sentence) }\end{array}$ & - & - & - & - & 2.57 & 2.3 & 4.12 & 2.16 & 3.37 \\
\hline
\end{tabular}

Average IU Density for Course B Texts: 2.9

COURSE C

\begin{tabular}{|l|l|l|l|l|l|}
\hline & \multicolumn{5}{|l|}{ Text Samples } \\
\hline & C1 & C2 & C3 & C4 & C5 \\
\hline $\begin{array}{l}\text { Idea Unit Density } \\
\text { (Average number of IU per } \\
\text { sentence) }\end{array}$ & 4.25 & 3.0 & 2.45 & 3.44 & 3.9 \\
\hline
\end{tabular}

Average IU Density for Course C Texts: 3.4

These results are yet more evidence of the differences between the texts used in the different disciplines. The Course $\mathrm{C}$ texts represent a higher concentration of Idea Units per sentence than the texts in Course B, which in turn are denser in ideas than the set textbook used in Course A. Accordingly, it is likely that Readers for whom conceptual density is a hindrance to comprehension would struggle with Course C Texts. Again, with no systematic comparison it is hard to say whether these academic texts possess 
comparatively high or low density of ideas, but suffice to say that when trying to create coherent understandings of text both at the micro and macro levels, the more Idea Units one has to process, the more difficult the task is likely to be.

\section{Chapter Summary}

Investigation of the nature of the authentic academic Texts profiled in this study has revealed that:

- Their nature differs between disciplines in terms of the lexical profiles of texts, the grammatical complexity and conceptual density.

- The average sentence length of all the Courses' Text samples was about 26 words per sentence. Given that sentence length is widely accepted as a fair indicator of grammatical complexity in sentences, the average length of sentences in Course $\mathrm{C}$ indicated that these Texts possessed the greatest syntactic Complexity and would thus be predicted to pose a challenge to many readers.

- In terms of conceptual density, measures of Idea Unit Density revealed that Course A possessed the least conceptual density (average 2.2 IU per sentence) and that Course $\mathrm{C}$ possessed the greatest, with an average of 3.4 Idea Units per sentence, which would suggest that these Course C texts may present greater challenge in terms of cognitive processing.

From the analysis of set texts in these first year Courses, it became apparent that a major difference between the disciplines was the extent to which their set texts were intended for the audience they were being read by in the current context. According to Chandler (n.d.), "embedded within texts are assumptions about the 'ideal reader', including their attitudes towards the subject matter and often their class, age, gender and ethnicity". This concept of the intended purpose/audience versus the actual purposes/audience of 
academic texts is one also explored by Coulthard (2001:4), who uses the terms "imagined readers" and "real readers". The argument is that the audience for whom a writer imagines they are writing (the "imagined readers") and the actual people who read texts in the course of their first year study ("real readers") may be quite different, and this gives rise to potential complications in the communication process from author to reader.

These concepts may help explain why the texts in some Courses may present considerably more challenge to first year student readers than the set texts in other Courses. The fact that the set text in Course A was a textbook written specifically for introductory level students entering the field at a tertiary level means that there is a high degree of overlap between the imagined and real readers, and thus, we would predict a context with fewer or less severe problems on the whole for the real readers. Whereas for Courses B and C, where the texts selected were intended for the author's peers and audiences primarily of the same academic standing as the author, there is a considerable gap between the imagined and real audiences. There is thus a higher likelihood of areas in which real readers may struggle. This probably accounts for a great deal of the variation observed between Course A and the other Courses B and $\mathrm{C}$ in this phase of Text analysis and also in my observations of the student Readers' struggles and experiences of the set texts.

The findings of this study support the position that academic text in higher education is characterized by highly abstract, academic and technical vocabulary (Brown, 2007). The Off-List (average 11\%) plus the AWL (average 10\%) items would account for up to an average of $21 \%$ of the lexical items in text. Thus, the fact that nearly a quarter of the lexical items in any given text would be likely to be academic or technical and specialist items is a strong indication of their potential difficulty. The coverage of the academic 
text samples provided by $\mathrm{K} 1$ and $\mathrm{K} 2$ combined was estimated to be on average about 78\%. It was also found that these texts, like those investigated by Pretorius (2006), and Brown (2007) do possess densely packed ideas, with the texts in some disciplines regularly containing more than three Idea Units per sentence. This density of ideas per sentence frequently serves to slow down and sometimes to confuse or distract readers. In addition to this, conclusions in the research literature concerning the complexity of the syntax of academic text have also been confirmed by the findings of this study, with confirmation of the existence of sentences with multiple dependent clauses and other complicating grammatical features.

Documenting the nature of academic Texts, acknowledging the degree of challenge that these Texts present, and viewing these in conjunction with the sets of demands and challenges each context and Task presents, provides a vital first step to challenging the notion that "any problems readers have with the text tend to be seen as deficiencies in the reader, deficiencies that are obviously compounded if the reader is not a native reader of the language of the text" (Coulthard, 2001:4).

The following Chapter presents the findings relating to the nature of the Tasks involved in first year Courses. 


\section{CHAPTER 6}

\section{ACADEMIC READING TASKS: FINDINGS \& DISCUSSION}

This Chapter presents the findings and discussion relating to Research

\section{Question 2:}

What is the nature of the typical academic reading tasks required of the reader in the first year of university study?

(a) What types of reading activities/outcomes/processes are required in a typical first year course in the Faculty?

(b) In a typical first year Course how do the reading task representations of lecturers and students compare?

(c) Given the characteristics of the texts and tasks, what are the characteristics of a 'good reader' in this Faculty?

The reading Tasks required of students in the first year Courses studied in this research have been documented and classified by means of three main qualitative methods: observation, interview and document review (Stake, 1995, p.114; Miles, 1994, p.9). This triangulation was undertaken to increase confidence in my interpretation of the central tasks and task representations of the various participant groups.

The findings are presented and discussed in the following sections:

(6.1) Course Outline content analysis

(6.1.1) Core generic first year academic reading tasks/processes/outcomes

(6.1.1.1) Reading for the three-part outcome

(6.1.1.2) Reading and critical thinking skills

(6.1.2) Core Course-specific academic reading tasks/processes/outcomes 
(6.1.2.1) Reading and the disciplines

(6.2) Interviews with Tutors/Lecturers \& Classroom Observations

(6.2.1) How Lecturers' and student Readers' perceptions of academic reading differ

(6.2.2) Reading and assessment

(6.3) Therefore, the characteristics of a 'Good Reader'

\section{(6.1) Course Outline Content Analysis: Reading Tasks/Outcomes/Processes in First Year Case Study Courses}

From the Course Outline analysis, both the explicitly stated and the implied reading tasks have been classified and grouped and what emerged from this process of analysis was a set of common core academic reading processes, and a further set of required reading processes that were unique to each particular Course studied. The terms 'tasks', 'processes' and occasionally 'outcomes' are used interchangeably in this context, and while they entail certain differences they represent the same line of thought - the all important purpose and expected outcome.

\section{(6.1.1) Core Reading Processes in the First Year Case Study Courses}

The core reading processes that overlapped in two or more of the three case study Course Outlines were as follows. 
Table 6.1: Core reading tasks/processes found to be overlapping in Courses A, B and $\mathrm{C}$

\begin{tabular}{|c|c|c|}
\hline $\begin{array}{l}\text { Found } \\
\text { in }\end{array}$ & Reading Task/Process (from Course Outline) & $\begin{array}{l}\text { Shared Core } \\
\text { Reading } \\
\text { Task/Process }\end{array}$ \\
\hline $\mathrm{A}$ & $\begin{array}{l}\text { Read to critically understand key material/ideas in } \\
\text { order to facilitate a tutorial session and generate } \\
\text { original ideas to contribute to the tutorial } \\
\text { group/discussion. } \\
\text { Read to understand the concepts/topics within } \\
\text { reading in order to be able to participate in tutorials. } \\
\text { Read to identify/understand key points/concepts in } \\
\text { set readings in order to be able to facilitate and } \\
\text { generate discussion with peers in tutorials. }\end{array}$ & $\begin{array}{l}\text { Read to participate } \\
\text { in tutorials }\end{array}$ \\
\hline $\mathrm{C}$ & $\begin{array}{l}\text { Read to identify/understand/learn/explore the } \\
\text { key/central issues/concerns/topics in the } \\
\text { field/discipline. } \\
\text { Read to deeply understand/learn foundational theory } \\
\text { and topics/concepts within the discipline. } \\
\text { Read to understand/learn/reflect on the key } \\
\text { concepts/theories/issues/research/topics in the } \\
\text { discipline, and be able to demonstrate this in written } \\
\text { assessment. }\end{array}$ & $\begin{array}{l}\text { Read to } \\
\text { understand/learn key } \\
\text { central topics } \\
\text { (and demonstrate } \\
\text { this in assessment) }\end{array}$ \\
\hline $\mathrm{C}$ & $\begin{array}{l}\text { Read to understand the links/associations between } \\
\text { topics in the field and how they can benefit each } \\
\text { other. } \\
\text { Read to understand how topics/concepts within the } \\
\text { discipline interact and affect each other. } \\
\text { Read to clearly identify/understand the } \\
\text { links/relationships between topics in the discipline } \\
\text { and how they affect each other. }\end{array}$ & $\begin{array}{l}\text { Read to } \\
\text { identify/under-stand } \\
\text { links/relation-ships } \\
\text { between topics in } \\
\text { the discipline. }\end{array}$ \\
\hline A & $\begin{array}{l}\text { Read to understand and learn key principles and use } \\
\text { them to analyse/identify key issues within case } \\
\text { studies. } \\
\text { Read to analyse the structure and context of case } \\
\text { studies from within the discipline. } \\
\text { Read to deeply analyse and evaluate cases/major } \\
\text { findings/situations/concepts in the discipline and be } \\
\text { able to express this in writing. }\end{array}$ & $\begin{array}{l}\text { Read to analyse case } \\
\text { studies from within } \\
\text { the discipline. }\end{array}$ \\
\hline $\begin{array}{l}\mathrm{A} \\
\mathrm{B}\end{array}$ & $\begin{array}{l}\text { Read to develop critical evaluation skills. } \\
\text { Read to develop key critical reading, analytical and } \\
\text { organizational skills for use in further study in the } \\
\text { discipline. } \\
\text { Read to develop key critical skills and use these }\end{array}$ & $\begin{array}{l}\text { Read to develop key } \\
\text { critical reading } \\
\text { skills. }\end{array}$ \\
\hline
\end{tabular}




\begin{tabular}{|c|c|c|}
\hline & $\begin{array}{l}\text { skills to critique a range of source material and } \\
\text { demonstrate this in written assessment. }\end{array}$ & \\
\hline $\begin{array}{l}\mathrm{A} \\
\mathrm{C}\end{array}$ & $\begin{array}{l}\text { Read to then be able to cite from these readings. } \\
\text { Read to be able to draw on literature and examples } \\
\text { effectively in written assessment. }\end{array}$ & $\begin{array}{l}\text { Read to be able to } \\
\text { draw on/cite } \\
\text { literature in written } \\
\text { assessment. }\end{array}$ \\
\hline B & $\begin{array}{l}\text { Read to understand and demonstrate this in weekly } \\
\text { quizzes. } \\
\text { Read to understand and remember in order to answer } \\
\text { in-class questions. }\end{array}$ & $\begin{array}{l}\text { Read to understand } \\
\text { and demonstrate this } \\
\text { understanding in in- } \\
\text { class quizzes. }\end{array}$ \\
\hline B & $\begin{array}{l}\text { Read to understand and generate one's own } \\
\text { findings/perspectives, and then to } \\
\text { communicate/demonstrate these in written form. } \\
\text { Read to generate original/innovative understandings } \\
\text { of/approaches to topics in the discipline. }\end{array}$ & $\begin{array}{l}\text { Read to generate } \\
\text { original/ innovative } \\
\text { perspectives/ } \\
\text { understandings/ } \\
\text { findings }\end{array}$ \\
\hline
\end{tabular}

These results demonstrate the existence of a core set of reading Tasks required of students in their first year in the Humanities and Commerce Faculties courses surveyed.

For ease of reference, these generic core reading tasks/processes were labelled with Roman numerals (I, II, III...), and in the following section the coursespecific reading tasks/processes have been labelled with an uppercase letter indicative of the course to which they belong, and lower case letters to differentiate them from each other (e.g. A(a), A(b)...). This labelling facilitated the analysis of the data collected in the student and staff interviews and during classroom observations, allowing the triangulation of data to occur. The resulting list of core academic reading tasks is shown below. 
Table 6.2: Core academic reading tasks common to case study Courses A, B and $\mathrm{C}$

\begin{tabular}{|l|l|}
\hline Label & $\begin{array}{l}\text { Core Reading Tasks/Processes/Outcomes common to all three } \\
\text { Courses }\end{array}$ \\
\hline I & Reading to Participate in tutorials. \\
\hline II & $\begin{array}{l}\text { Reading to } \text { Understand/learn key central topics (and demonstrate this in } \\
\text { assessment). }\end{array}$ \\
\hline III & $\begin{array}{l}\text { Reading to Identify/understand links/relationships between topics in the } \\
\text { discipline. }\end{array}$ \\
\hline IV & Reading to Analyse case studies from within the discipline. \\
\hline V & Reading to Develop key critical reading (and thinking) skills. \\
\hline
\end{tabular}

In the remaining shared core reading tasks/processes, there is an overlap between Courses B and C and between Courses A and C.

Table 6.3: Core academic reading tasks shared by two courses

\begin{tabular}{|l|l|}
\hline Label & Core Reading Tasks/Processes/outcomes shared by two Courses \\
\hline BC(a) & $\begin{array}{l}\text { Reading to Generate original/innovative perspectives/understandings/ } \\
\text { findings. } \\
\text { (core to both Courses B and C) }\end{array}$ \\
\hline AC(a) & $\begin{array}{l}\text { Reading to Be able to draw on/cite literature in written assessment. } \\
\text { (core to both Courses A and C) }\end{array}$ \\
\hline
\end{tabular}

These key core academic reading Tasks were found to represent the academic reading processes and outcomes of:

- recall

- linking

- analysis and application

- critical thinking

- development of original thought 
(6.1.1.1) Reading for the Three-part Outcome: (i) Content development, (ii) Skill development, and (iii) Thinking development

In addition to observing the core generic academic reading tasks/outcomes, the findings of this study suggest that reading in the first year of study in the Humanities and Commerce Faculties at this University appears to be primarily geared towards learning central foundational content and the links and patterns existing within it for the purpose of factual recall in assessment and classroom participation. In addition to this, it also appears that an expected outcome of reading is the development of key skills such as analysis. Furthermore, reading is expected to bring about the development of critical and original thinking patterns and processes. The core generic academic reading Tasks in the first year Courses studied all represented academic reading tasks that aimed to develop particular content, and/or specific skills, and/or certain types of thinking. These are indicated in Table 6.6 below.

Table 6.4: Core generic academic reading tasks for courses categorised into content, skills or thinking development

\begin{tabular}{|l|l|l|l|}
\hline \multirow{2}{*}{$\begin{array}{l}\text { Academic reading } \\
\text { Task/Outcome } \\
\text { in first year study }\end{array}$} & \multicolumn{3}{|l|}{ Classification } \\
\cline { 2 - 4 } & $\begin{array}{l}\text { Content } \\
\text { Development }\end{array}$ & $\begin{array}{l}\text { Skills } \\
\text { Development }\end{array}$ & $\begin{array}{l}\text { Thinking } \\
\text { Development }\end{array}$ \\
\hline recall & $\checkmark$ & & \\
\hline linking & $\checkmark$ & $\checkmark$ & \\
\hline analysis and application & $\checkmark$ & $\checkmark$ & \\
\hline critical thinking & $\checkmark$ & $\checkmark$ & $\checkmark$ \\
\hline $\begin{array}{l}\text { development of original } \\
\text { thought }\end{array}$ & $\checkmark$ & $\checkmark$ & $\checkmark$ \\
\hline
\end{tabular}

The expected outcome of content development is in itself deceptively simple, as the research reported in this thesis highlights what a fraught and complex process meaning-making from text can in fact be. The second category, reading to develop skills, is also usefully viewed as the development of 
strategy knowledge and strategic ways of dealing with text. The third category, the development of types and ways of thinking, is also extremely complex and greatly affected by the particular discipline in which a student is studying. There is scope for considerably more research into the nature of the skill and thinking development required of students through the act of academic reading, and how this is expected to occur within specific disciplines.

Having more closely identified this, we would then be in the position to begin to observe and design pedagogical processes that Lecturers and Tutors could utilise to ensure that academic reading does in fact bring about not only content development but also works systematically to engender the expected skill and thinking outcomes in a strategic and informed manner.

The importance of greater systematic understanding of the link between academic reading and the development of these skills and ways of thinking is reinforced by related US research that found that " $83 \%$ of faculty say that the lack of analytical reading skills contributes to students' lack of success in a course" (ICAS, 2002, p.4). Great importance is thus ostensibly placed on the ability of students to develop and demonstrate these reading based skills and ways of thinking, but how much explicit thought is given to the manner in which these are to be developed? Also raised is the question of what balance (proportionally) is given to these three equally important outcomes in current Course design?

It appears probable to me that a strong empirical basis for the postulation of specific reading skills/strategies as crucial to the higher education experience has yet to be established in the New Zealand context. What is more, while it is frequently bemoaned that students are under-prepared for the demands of 
academic literacy, what exactly it is that they are unprepared for has, by and large, perhaps not been as accurately and clearly specified as it ought to be. Or, where it has been attempted, it may still be largely the result of 'educated guesses' and 'conceived wisdom' and often in generic terms without full heed given to the impact of discipline.

In the documentation of the demands of academic reading in higher education, a crucial but sometimes overlooked factor is acknowledgement of the contextdependent nature of literacy and the existence of situated and often socioculturally generated conceptions of reading within specific universities, faculties, schools, courses and even classes. There is therefore a need for institutions to become explicitly aware of their own implicit 'cultures of reading' in order to better assist readers wishing to operate successfully within it.

\section{(6.1.1.2) Reading and Critical Thinking Skills}

The Course Outline analyses have also revealed that across all three main case study Courses a particular assumption was held that one of the core outcomes of academic reading of the set texts would be the development of critical thinking skills. However, the relationship between Reading, Critical Reading, and Critical Thinking in both the research literature and practice is far less

clear cut. On the one hand White (2004) views critical thinking as a "component of critical reading", while on the other, Ward and Beach (2007, p.9) perceive critical literacy (including reading) to be a subset of critical thinking. Both the research literature and the findings of this study indicate that these constructs should not be blurred in higher education. 
Past experience and close observation of students in this study suggests strongly that it is erroneous to say that 'reading' and 'critical reading' are synonymous, or in some cases that 'critical reading' is the same thing as 'critical thinking'. A truer representation of the relationship between these three commonly blurred entities is likely to be that they may be separate processes, and that when reading occurs concurrently with critical thinking then this can be called the process of critical reading. Thus, the exercise of critical reading reinforces and exercises critical thinking skills, but importantly, reading alone is most certainly no guarantee that critical thinking will occur.

The importance of this argument to the findings of the Task analysis in this study is that reading to develop key critical reading and thinking skills (V) was found to be a core generic academic reading Task. This common core Task was repeated in all three Courses, and its prevalence (perhaps university-wide) represents a potentially flawed belief held by Course Coordinators; namely that by amassing largely unguided reading experiences in the discipline a student will/can develop critical reading skills. This underlying belief not only assumes common starting and end points for all students, but more importantly, it represents the idea that repeated, independent exposure to the academic text is the means by which to bring about the development of sophisticated thought and practice. This assumption is analogous to, and equally as untenable as, the idea that the act of repeatedly throwing a person in deep water is sufficient to enable them to quickly and successfully become an expert swimmer. One would be hard pressed to find empirical support for such an assumption.

Rather, it is likely that readers, "contrary to what we seem to hope, do not inherently possess or 'naturally' develop" (Railton \& Watson, 2005, p.183) 
critical reading skills. Yang and Wilson (2006, p.367) echo this sentiment, stating that "we need to stop teaching reading by simply practising reading". Similarly, Wilson $(2009$, p.3), in reflecting on her English language teaching experience, comments on the fact that "teachers may be tempted to hope that simply 'practise, practise, practise' will build students' reading ability". Their discussion was in the context of the foreign language classroom, but applies just as well in the general higher education context.

It should be acknowledged that reading can and does promote the development of certain thinking skills, since the successful processing of certain types of text favours the exercise of certain information processing processes, and to get better at something one must actually do it. However, the act of reading alone is not sufficient for the development of higher order thinking skills, since the choice of texts, the tasks and processes, the degree of guidance and instruction, and a wide array of reader characteristics also play an important role. Given that the staff in this study testified to giving to students in their respective courses generally small and greatly varying degrees of direct instruction, modeling and guidance, it simply cannot be assumed that the necessary conditions exist to ensure that the expected reading outcomes are achieved. The case study student Readers' academic results in these Courses appear to corroborate this.

So how, in fact, can critical reading skills be developed? While Thistlethwaite (1990, p.587) states that "[d]efinitions of critical reading vary greatly", despite this, research and literature on the matter appears to almost unanimously support the ideas that (i) such higher level skills and thinking processes are developmental in nature, (ii) they are contextual (situated within social and cultural contexts), and (iii) that their improvement requires repeated opportunities for guided practice. 
From my reading I have compiled a list of what appear to be keys to the development of critical reading in higher education:

- a questioning mindset, a 'suspicious eye', a healthy skepticism that does not accept the printed word at face value and does not wish to be manipulated by text (Wallace, 1995; Thistlethwaite, 1990)

- $\quad$ an attitude of waiting, reflecting on text, being flexible in one's opinions and withholding or suspending judgment until all sides of the issue have been explored and evaluated (Thistlethwaite, 1990, p.587; Ward \& Beach, 2007; Kemp, 1963)

- use of background knowledge to evaluate what one reads and from this to form one's own opinion (Thistlethwaite, 1990)

- $\quad$ use of metacognition (Pithers \& Soden, 2000)

- use of recognized patterns of argument and higher levels of questioning designed to assist one to reach one's own conclusions (Ward \& Beach, 2007, p.9)

- an appreciation that texts are never neutral (Wallace, 1995; Yang \& Wilson, 2006; Cervetti, Pardales, \& Damico, 2001).

It is important to understand that in fact behind each of these lies a set of values, experiences and beliefs about text that a reader must possess or espouse before this action will become natural to them. It is in this core set of beliefs, values and experiences that we may find a number of challenges for the Pacific student. For instance, an awareness of the author is necessary for an approach to text that challenges the authority of the writer and the realization that each author has their own agenda (1 and 6). The use of background knowledge of course requires the existence of such relevant and rewarded schema (3). Moreover, the use of patterns of argumentation assumes that one knows these patterns (5). However in this study, observation of the 199 
Readers has shown that these qualities and characteristics simply cannot be assumed to exist upon starting tertiary education.

\section{(6.1.2) Core Course-Specific Reading Expected Outcomes in First Year Courses}

As previously mentioned, despite having common core generic Tasks, the Courses were also clearly distinguished by the existence of remaining reading outcomes/processes/tasks that were specific to each Course. The core reading processes/tasks/outcomes found not to overlap but rather to be specific to Courses A, B and C in this study are presented in turn below. 
Table 6.5: Core course-specific academic reading tasks specific to Courses A, B and C respectively

COURSE A

\begin{tabular}{|l|l|}
\hline Label & Reading task/process specific to Course A \\
\hline A(a) & $\begin{array}{l}\text { Read to understand/critically analyse theories/concepts in order to be able to } \\
\text { recognize/interpret/examine instances of their application in real life } \\
\text { situations/events/behaviours. }\end{array}$ \\
\hline A(b) & $\begin{array}{l}\text { Read to form a basic understanding to then have this built upon/extended } \\
\text { (in lectures and further learning). }\end{array}$ \\
\hline $\mathrm{A}(\mathrm{c})$ & Read to gain knowledge to apply/demonstrate in group work. \\
\hline $\mathrm{A}(\mathrm{d})$ & $\begin{array}{l}\text { Read to identify and describe concepts/theories/ideas that are relevant to a } \\
\text { specific given topic. }\end{array}$ \\
\hline $\mathrm{A}(\mathrm{e})$ & $\begin{array}{l}\text { Read to understand and integrate a range of concepts/theories and be able to } \\
\text { apply them appropriately to other specific situations. }\end{array}$ \\
\hline $\mathrm{A}(\mathrm{f})$ & Read to engage in meaningful discussion with the lecturer/tutor. \\
\hline $\mathrm{A}(\mathrm{g})$ & Read to identify critical steps necessary to accomplish certain tasks. \\
\hline $\mathrm{A}(\mathrm{h})$ & Read to learn/understand the broader context in which theories are relevant. \\
\hline
\end{tabular}

COURSE B

\begin{tabular}{|l|l|}
\hline Label & Reading task/process \\
\hline B(a) & $\begin{array}{l}\text { Read to recognize/understand/learn and critically assess the foundational } \\
\text { methods/tools within the discipline, and how they are used. }\end{array}$ \\
\hline B(b) & Read to be able to develop one's own essay writing assignment. \\
\hline B(c) & $\begin{array}{l}\text { Read to compare topics within the discipline, and recognize the current and } \\
\text { historical diversity that exists within the discipline. }\end{array}$ \\
\hline COURSE C \\
\hline Label & Reading task/process \\
\hline C(a) & $\begin{array}{l}\text { Read to explore/research and understand a specific issue within the } \\
\text { discipline and demonstrate this in written assessment. }\end{array}$ \\
\hline C(b) & $\begin{array}{l}\text { Read to identify/understand initiatives to overcome problems/issues and to } \\
\text { select the most appropriate from amongst them for addressing a given issue }\end{array}$ \\
\hline C(c) & $\begin{array}{l}\text { Read to understand and remember key concepts in the set readings in order } \\
\text { to make connections between readings and the material presented in } \\
\text { lectures. }\end{array}$ \\
\hline C(d) & $\begin{array}{l}\text { Read to identify/understand the views/philosophies/groundwork of specific } \\
\text { authors on a given topic and assess the extent to which these authors' views } \\
\text { may interrelate. }\end{array}$ \\
\hline C(e) & $\begin{array}{l}\text { Read to understand a given topic, develop an argument and then express } \\
\text { this in writing. }\end{array}$ \\
\hline C(f) & Read to visualise concepts. \\
\hline C(g) & $\begin{array}{l}\text { Read to identify/understand various authors' perspectives on critical factors } \\
\text { within discipline topics. }\end{array}$ \\
\hline C(h) & $\begin{array}{l}\text { Read to understand various pieces of research on a given topic and } \\
\text { synthesize an understanding of the issue. }\end{array}$ \\
\hline
\end{tabular}


Thus, it can be seen that what starts to differentiate the expected outcomes of academic reading in the various disciplines has to do with the modes of text interaction that they promote. These lists appear to demonstrate the underlying values and beliefs that constitute what it means to read in that Course. The lists above constitute appreciably different expected outcomes, and are rooted in much deeper beliefs about what it means to be a scholar in this discipline and how to interact with texts the way that a [discipline]ist does.

\section{(6.1.1.3) Reading and the Disciplines - Support for the Context- dependent Nature of Academic Reading Literacy}

The difference between the core approaches and orientations in the Courses is further evidence that academic reading is highly context dependent. As mentioned, Course A involved reading to understand and apply theory to case studies and novel situations. In this first year Course one must be apprenticed into the 'speak' of the field, learning how to use the terminology and how to view everyday situations and events through the lenses of the many theoretical positionings that combine to make up this discipline. It appears that for Course A, the expected reading processes are primarily concerned with the understanding and application of concepts/theory, and the analysis and observation of scenarios in order to identify the particular concepts/theories that have been operating in that situation.

In Course $\mathrm{B}$, however, the core reading approach was to read to understand and compare cases of the topics under investigation in contexts across the world and throughout its documented history. The orientation was to understanding case studies; developing analytical skills; learning and understanding foundational methodology for study in the discipline; appreciating the nature of the discipline; comparing topics; developing skills 
necessary for further study in the field; and developing one's own ability to devise and research relevant topical issues from those readings. By reading across contexts, students were expected to devise frameworks through which to comparatively view the phenomena under investigation. While engaging with the content and becoming familiar with the key theories and theorists in the field, and their various approaches to the discipline, it was also desired that students would develop an understanding of how topics are treated in scholarly situations. This Course required that students should read with an open mind, ready to give value to the many and diverse viewpoints and thus begin a journey towards an understanding of the discipline, how people over the decades have studied it, and how it is continually shaped by the happenings of today's world. For Course B, the expected reading processes were primarily concerned with the understanding and comparison of cases, the development of core skills for future study in the field, and understanding the historical context and diverse nature of the discipline.

The core reading approach in Course C, however, was to read to understand, synthesize and critique. It was desired that students should read with an orientation to deep thinking, reflection, visualisation and the evaluation of concepts; the demonstration of understanding in assessment; identification and description of the views/philosophy of particular authors; comparison and synthesis of the views of various authors on specific topics; building an understanding of a topic and relating it to contemporary issues; the evaluation and choice of the best solutions to issues; making connections between readings and lectures; and the research of specific topics. For Course $\mathrm{C}$, the expected reading processes were primarily concerned with deep thinking about concepts, their value when compared to the ideas of other theorists, and their value when compared to the student reader's own world knowledge and experience. This critical thinking, synthesis and evaluation was expected to be 203 
demonstrated in assessment. It was also concerned with relating theory to contemporary issues, judging its value in novel contexts, and searching for solutions to contemporary problems in the field.

Thus, in one of the first year Courses studied, Readers must read to apply, in another they must read to compare, while in another they must read to critique. In this way the differences of focus between first year courses begins to be seen and, since it is clear that to 'understand and apply' is a more straightforward activity than to 'critique', it suggests that student experiences of the degree of challenge presented to readers in different courses within ostensibly the same level of tertiary study the tertiary system may be an area warranting further investigation. It is also an indicator that while a core set of generic reading skills may exist, the expectations and experiences of reading in the various courses and in different disciplines are likely to be considerably varied.

These findings are consistent with a small but growing research evidence base which documents the links between discipline and academic reading, and this has been touched on in the literature review. Most notable was Kirkpatrick and Mulligan's (1996) investigation of the reading requirements and attitudes to reading across the disciplines of Engineering, Health Science and Social Science, which revealed that there are significant differences in what is believed to be "appropriate reading practices" at undergraduate levels across the various disciplinary areas.

The existence of generic and possibly teachable approaches to academic text is an exciting but dangerous thought. It has the potential to empower educators and students within learning contexts that are centered on the use of academic texts for the storage and transmission of ideas, while at the same time it 204 
possesses the risk inherent in any form of skills documentation - the slide into standardizing, benchmarking, and the creation of the false reality of a rigid and fixed mode of text interaction and literacy development. This would be potentially destructive in a setting such as higher education which strives for a flexible, dynamic, strategic, thoughtful approach to text, leading to innovation and original thought and academic endeavour indicative of an individual's true potential.

Thus, what is made of these results and any future research in the area must achieve a proper recognition of the context-dependent nature of reading literacy and the importance of the study of literacy within its specific societal and cultural context (Fiocco, 1996, p.1). To balance this, it must also be responsive to the need for the freedom of individuals to pursue interaction with text to, for, and by their own means. I would argue that novice readers in higher education require a great deal of scaffolding, provided by an academic advisor, tutor, or mentor who is sensitive to the individual needs of the readers and supports their development from 'student' to 'scholar' in the discipline.

The next step in this research trajectory would be to further and more closely define the possible mechanisms by which these reading Tasks/processes are successfully accomplished by a wide range of readers. This investigation would be based on the premise that the reading Task at hand (or at least one's own mental representation of that Task) determines one's strategic approach to academic reading and therefore the mechanisms by which one makes meaning from and interacts with text. As Nist and Simpson (2000, p.648) point out, "students who know how to interact with texts and how to determine tasks independently can use this information to assist them in strategy selection". It is these mechanisms that can be brought to readers' conscious awareness and, under guided practice, can be incorporated into their modes of meaning205 
making from text when their task representations and situated conceptions of reading and learning become more closely attuned to those of the discipline.

A similar concept to what I refer to as 'mechanisms', Hermida (2009, p.5) calls "categories of analysis", and argues that each discipline "has also its own specific categories of analysis, which need to be taught". These categories document ways of interacting with academic text that are consistent with the cultures of reading and the epistemological positions of the various disciplines. Hermida's (2009) categories of analysis provide the student reader with explicitly defined points/areas for focus which are usually not known to first year students, and thus greatly assist them to interact with texts. Yang and Wilson (2006, p.367) echo the importance of providing a context and clear purpose for reading, stating that students need to have a clear idea why they are reading and how this text relates to other Course aspects and that a teacher must

[m]ake sure that the students know which reader role you want them to adopt, whether that be making meaning, exploiting the text for useful vocabulary, looking at the text as a model for some other task, learning some new information in preparation for an assignment, finding out the author's opinion on the topic, or do you expect them simply to enjoy the story?

Hermida's (2009) solution to the problem first year students face in not knowing how to appropriately interact with academic text in their discipline is for lecturers in each discipline to work to actively instill both "general analytical tools" and "discipline-specific values and strategies" which will assist the students to read and learn within the discipline. In this way, guided and scaffolded by an 'expert' reader in the field (who also usually happens to be their marker) a novice reader may transition faster and more smoothly into 
the context-dependent features of academic reading within the Course/wider discipline, and be enabled at an earlier point to begin to express their academic potential.

\section{(6.2) Interviews with Tutors/Lecturers, and Classroom Observations}

Using interview data collected from student and staff participants a comparison of the task representations of Lecturers and student Readers was carried out and, when analysed in conjunction with classroom observation notes, revealed insights into the similarities and differences between Lecturers' and students Readers' focus and understandings of academic reading in their disciplines.

In coding and summarizing the interviews with the Course A Tutor and matching this with the set of core academic reading Tasks identified in the Course Outline analysis, the reading Tasks/Processes that were most prominent in Lecturer/Tutor's perceptions were those outlined in Table 6.8 below.

Table 6.6: Task labels for coding of lecturer/tutor interview transcript data for task analysis

\begin{tabular}{|l|l|}
\hline $\begin{array}{l}\text { Task } \\
\text { Label }\end{array}$ & Description \\
\hline II & $\begin{array}{l}\text { The generic task of reading to understand/learn key topics (and } \\
\text { demonstrate them in assessment) }\end{array}$ \\
\hline III & $\begin{array}{l}\text { The generic task of reading to identify/understand the links/relationships } \\
\text { between topics in the discipline }\end{array}$ \\
\hline A(a) & $\begin{array}{l}\text { The Course-specific task of reading to understand theories/concepts and } \\
\text { recognize/examine instances of their application }\end{array}$ \\
\hline A(b) & $\begin{array}{l}\text { The Course-specific task of reading to form a basic understanding and } \\
\text { have this built upon/extended in lectures and further learning }\end{array}$ \\
\hline
\end{tabular}


While the reading Tasks/Processes most prominent in an analysis of the student Readers' interviews were as outlined in Table 6.9 below.

Table 6.7: Task labels for coding of student readers' interview transcript data for task analysis

\begin{tabular}{|l|l|}
\hline $\begin{array}{l}\text { Task } \\
\text { Label }\end{array}$ & Description \\
\hline II & $\begin{array}{l}\text { The generic task of reading to understand/learn key topics (and } \\
\text { demonstrate them in assessment) }\end{array}$ \\
\hline A(b) & $\begin{array}{l}\text { The Course-specific task of reading to form a basic understanding and } \\
\text { have this built upon/extended in lectures and further learning }\end{array}$ \\
\hline
\end{tabular}

The reading Tasks/Processes most prominent in the analysis of the field notes made during the Classroom Observations were those listed in Table 6.10 below.

Table 6.8: Task labels for coding of classroom observation data for task analysis

\begin{tabular}{|l|l|}
\hline $\begin{array}{l}\text { Task } \\
\text { Label }\end{array}$ & Description \\
\hline I & The generic task of reading to participate in tutorials \\
\hline II & $\begin{array}{l}\text { The generic task of reading to understand/learn key topics (and } \\
\text { demonstrate them in assessment) }\end{array}$ \\
\hline A(a) & $\begin{array}{l}\text { The Course-specific task of reading to understand theories/concepts and } \\
\text { recognize/examine instances of their application }\end{array}$ \\
\hline
\end{tabular}

\section{(6.2.1) How Lecturers' and Student Readers' Perceptions of Academic Reading Differed}

A comparison of the main themes emerging from interviews revealed a degree of disparity between the beliefs of the Lecturers/Tutors and those of the student Readers, regarding the ways of reading in the Course. These results suggest that the primary focus of the staff members was on the reading 
processes/tasks associated with gaining mastery of content. On the other hand, the students' focus appeared to be geared primarily to the reading tasks/processes involved with the assessment-related or performance-related outcomes.

This difference is significant but not surprising, and concurs with what has been previously documented. Railton and Watson's (2005, p.184) main finding for example, was that students' conceptions of what "doing the reading" entailed did in fact differ considerably from teachers' conceptions. Railton and Watson argue that the natural consequence of lecturers' expectations regarding the purposes and processes of reading remaining largely unspoken is that students' understandings of theirs and the lecturer's roles in university closely mimic the roles they observed in classrooms from lower educational levels. Similarly, Yang and Wilson (2006, p.367) also observe that "students have expectations of how texts can and should be used based on their prior experience of texts as social practice", and they too stress that teachers must "encourage and facilitate students' use of texts in new social contexts".

While differences between Lecturer/Tutor and student Readers' perceptions of reading may not come as a surprise, what was potentially more surprising was that amongst the group of students surveyed (all of whom self-identified as coming from the same broad ethnic background) considerable diversity existed in their beliefs about reading, the role of reading in this Course and the role of the student reader was found. In Course A, two male and two female students (S4(M), S12(M), S7(F) and S13(PF)) were interviewed at the end of the Course to ascertain their perceptions of reading in Course A. Moreover, while the students all believed that reading played an important role in this Course, interviews revealed that their uses of the set text (a textbook) were, for 
the most part, relatively limited. Amongst the Readers the only time it could be said for certain that all the students had interacted in a systematic way with the textbook was for assessment tasks.

Nist and Simpson (2000, p.9) stress that

it is important for students to become aware of their epistemologies about learning and to realize that their professors may have differing belief systems. General or domain-specific beliefs about learning are important because they affect students' choices regarding how they read, process and choose to study.

The study has shown that beliefs varied quite widely amongst the four student Readers about the necessity of reading in preparation for lectures and tutorials. They also differed greatly in the variety of strategies or skills they believed were useful for reading in this Course. S4(M) relied heavily on close reading for subject knowledge due to a perceived lack of background information on the topic, while S7(F) discussed the importance of common sense and a memorizing approach to the text. For S13(PF) reading aloud was the key to understanding concepts, and to S12(M) the discussion of topics and debate about them amongst peers was the best technique for learning from text. In terms of an understanding of what it meant to read in this discipline, S12(M) and S13(PF)'s understandings most closely resembled the Tutor's conception of reading, but none of the Readers appeared to have developed a full understanding of what was required of them. They did not possess an understanding of texts as situated within the wider field and as the contributions of members of an academic community spanning time and location. 
In research on the definitions of tertiary literacy (albeit predominantly writing) Fiocco (1996) report that the "discrepancy between what staff and students perceive as concerns in literacy, demonstrates the need for more co-operation and communication between staff and students." These findings and my survey of literature on this matter point clearly to the idea that Railton and Watson, (2005, p.183) express so well; that "students and teachers are two communities divided by a common language". They point out that while lecturers may (or as I have observed, may not) know what they mean when they ask students to "go and read", in effect "such proclamations are in themselves practically useless to new students unless they are accompanied by further explanation such as, what to read, how to read it, and what to do with it".

Fiocco (1996, p.6) stresses that in order for teaching staff to have a "more meaningful" definition for their own pedagogical practices within their discipline, they need to engage in consideration and discussion of the literacy demands and requirements specific to their disciplinary context. It is therefore vitally important for Lecturers/Tutors and Course Coordinators to enter into the investigation of reading Tasks involved in their own Courses and in the discipline as a whole. Moreover, there is great need to communicate these expectations to students with much greater clarity than is currently done (Railton \& Watson, 2005, p.183). However, as the lecturer responses in Fiocco's study predominantly indicated, the common perception was that there was simply not enough time for lecturers to be involved in the teaching or development of higher academic literacy skills. Nevertheless, as Fiocco (1996, p.6) points out, "theories on the instruction of tertiary literacy demand discipline lecturer involvement". 
The situation is not completely lost though, since there is evidence that the implicit understandings of lecturers can be brought to their own, their colleagues' and their students' explicit understandings, and student readers' task representations can be shaped and fashioned. This appears to be best achieved by attending to the breadth and scope of the readers' conception of what it means to study (learn from text) in this discipline. In doing so an educator can, as the Course B Lecturer observed, "discipline the imagination" of student readers, empowering them and further aligning their task conceptions with those of the Course and the wider discipline - given of course that these have first been given proper collective consideration and scrutiny. "If students can learn to think about the cognitive and metacognitive processes essential to understanding a text or task, they then can define their goals and proceed appropriately (Hadwin \& Winne, 1996; Pressley, 1995)" (Nist \& Simpson, 2000, p.535), and whether or not this constitutes a demanding and time consuming process (as is the usual objection to its undertaking) is likely to depend on how it is carried out.

\section{(6.2.2) Reading and Assessment}

The 'forcing' of reading by tying it to assessment was a strategy quite evident in the first year Courses surveyed. The tying of reading to assessment was apparent in (1) the Course Outlines (wherein assessment tasks such as reading log type learning journals were required), (2) the interviews with Lecturers and Course Coordinators, (wherein the Course $\mathrm{C}$ lecturer and Course A Tutor both spoke directly of the necessity of doing so to make sure that at least a certain percentage of the set readings were actually read by students), and (3) the classroom observations undertaken (wherein the in-class presentation of set readings' content was required of students). 
The deliberate and strategic tying of reading to assessment is consistent with Railton and Watson's (2005, p.188) report that “assessment is the most significant prompt for learning' (Boud, 1995, p.37) and the principle way of 'getting students to spend time on task' (Gibbs, 1999, p. 45)." These findings also strongly support Hermida's (2009) observation that

while all three components of the system - objectives, teaching and learning activities, and assessment - are important, assessment is the one that plays the most influential role in students' decision on whether to take a deep or surface approach to reading and learning (Gibbs, 1999 \& Forsaith, 2001). Students are heavily influenced by the hidden curriculum. They look for clues and use these to drive their study effort. Very little of out-of-class student learning is unrelated to assessment.

(Hermida, 2009, p.8)

In the cases chosen for Task analysis in this study it is apparent that there are considerable differences in the types of assessment used, and that this has a direct effect on the reading loads and demands of the course, which in turn affects the type of reader-task interaction expected and that which was actually enacted. The role of reading in the assessments of the various Courses strongly influenced and shaped the ways in which students did (and did not) interact with the set texts. Educators within this higher education context are thus faced with the dilemma of needing to induce reading by tying it to assessment, while despairingly observing the natural consequence of Readers' pragmatic response of only reading for assessment. This represents an unhealthy scenario which requires much further research attention.

\section{(6.3) Therefore, the Characteristics of a 'Good Reader'}

In the previous Chapter the characteristics of the academic Texts in this educational context were documented and in this chapter the core Tasks have 
been identified. In light of these characteristics it is now possible to describe a 'Good Reader' in these Courses. For the sake of brevity I will focus on what it means to be a 'Good Reader' in Course A.

In Course A, a 'Good Reader' would be one who was able to successfully and independently comprehend (and remember information from) texts which were high in academic, technical and specialist vocabulary, were grammatically complex, dense in ideas, and with highly varying types and amounts of non-linguistic features. In addition, they would need to be able to appreciate that more often than not, they are not the intended readers of the texts they are required to read. Furthermore, as well as possessing the ability to effectively and efficiently accomplish generic core academic reading tasks, they would also possess an accurate appreciation of and ability to accomplish sets of specific core reading Tasks relevant to each of the Courses they are enrolled in.

Thus, a 'Good Reader' in Course A would be able to:

- read to be able to participate in class discussion,

- understand key discipline concepts (and being able to demonstrate this in assessment),

- recognise links between ideas, and

- analyse the content of readings and engaging with them critically.

In addition to this the good reader would also read to be able to:

- recognize, interpret and examine instances of their application in real life

- form a basic understanding to build on in lectures

- gain knowledge to apply/demonstrate in group work

- judge the relevance of concepts/theories/ideas to a given topic

- apply concepts/theories appropriately to other specific situations.

- engage in meaningful discussion with the lecturer/tutor. 
- identify critical steps necessary to accomplish certain tasks.

- learn/understand the broader context in which theories are relevant.

According to the Course A Tutor, a 'Good Reader' would read to be able to grasp basic concepts and theories and determine their applicability in novel contexts. They would read to acquire the terminology of the field and would read beyond the set texts, while constantly seeking to integrate topics and look for the connections between them. The demands of observed contact time (class time) required that a 'Good Reader' read with an orientation to deep understanding, long term remembering, quick recognition and thoughtful application of ideas in new contexts.

Thus, by drawing together the various analyses, the profile of a prototypical 'Good Reader' has emerged, which will be later referred to in order to assess the 'match' between the ideal reader in this Course and the current characteristics of the Pacific Readers. Some of these core characteristics also represent a 'Good Reader' in the other case study courses, but the findings do suggest that being a good reader in Courses B and C would additionally entail the successful completion of other course-specific academic reading tasks and processes. It is also clear that the Lecturer's own interpretations of what it takes to be a good reader have shaped their expectations of students, the ways they design the learning and teaching activities, and the assessment tasks that involve academic reading in the course. These expectations can vary considerably, even amongst courses taught by different lecturers within the same discipline.

\section{Chapter Summary}

This phase of the research set out to document the nature of the typical academic reading Tasks required of readers in the first year of university study 
in the chosen Faculties. It was found that a core set of generic academic reading Tasks existed across the courses selected from the six for closer study. It was also found that sets of core academic reading Tasks specific to each course existed. Identification of these provided a basis for conceptualizing the central reading approach in each of the courses. The three courses each represented different central reading approaches and 'cultures of reading'.

The varying difficulty of these central reading approaches raises the question of comparability of the first year reading experience of students across the University. It is suggested that this has implications particularly in light of current movements toward the mapping of pathways of learning in this higher education context and the documenting of core academic skills.

In these typical first year Courses a substantial difference in focus between the beliefs of the Lecturers and student Readers about what reading in that Course entailed was found. While Lecturers'/Tutors' main perceptions of what constituted academic reading in their Course centered on the development of core disciplinary content knowledge, the student Readers' conceptions of what constituted reading in the Course invariably centered on doing what it took to complete assessment.

Given the characteristics of the Texts and Tasks as established in this and the previous phase of the study, the characteristics of a 'Good Reader' specifically in Course A were able to be documented.

Such an orientation to Text, determined by the Tasks identified in this chapter, guides the processes by which a Reader interacts with the set texts and seeks to make meaning from them. This and the previous chapter have shed light on the nature of the Texts and Tasks within the context of this University and the 216 
chosen Faculties. Understanding these two factors in the Text-Task-Reader interplay in effect establishes the context of the Reader. The next step was to identify the nature of the Pacific Readers themselves. The following chapter presents the aggregate findings of investigation into the nature of these Pacific student Readers as a group within their first year of tertiary study. 


\section{CHAPTER 7}

\section{THE PACIFIC READERS: FINDINGS \& DISCUSSION}

The findings and discussion in this Chapter address Research Question 3(a):

What are the characteristics of Pacific students as academic readers in the first year of university?

(a) What are the characteristics of first year Pacific students in terms of:

(i) Salient reading experiences throughout their reading history (Socio-cultural and literacy background)

(ii) Vocabulary size (as an aspect of language proficiency related to academic reading)

(iii) Reported patterns of reading strategy use, habits and attitudes

(iv) Reading speed (as an indication of fluency)

The profiled Reader characteristics that are reported in this chapter are:

(7.1) Pacific Readers' reading backgrounds and histories (based on salient memories recalled during the interviews)

(7.2) Readers' Vocabulary sizes

(7.3) Readers' Reading Rate

(7.4) Readers' reported academic reading Strategies, Habits and Attitudes

\section{(7.1) Themes from Pacific First Year Students' Reading Histories \& Memories}

In all, 14 interviews were carried out with first year students of Pacific descent. Demographic information gathered from the research participants showed that the group possessed the following characteristics: 
Table 7.1: Pacific student readers' demographics and language backgrounds

\begin{tabular}{|c|c|c|c|c|c|c|}
\hline Reader & $\begin{array}{l}\text { Place } \\
\text { of } \\
\text { Birth }\end{array}$ & $\begin{array}{l}\text { Age } \\
\text { group } \\
\text { range }\end{array}$ & $\begin{array}{l}\text { Home } \\
\text { language/s }\end{array}$ & $\begin{array}{l}\text { Home } \\
\text { language } \\
\text { used with } \\
\text { parent/s }\end{array}$ & $\begin{array}{l}\text { Home } \\
\text { language } \\
\text { used with } \\
\text { sibling/s }\end{array}$ & $\begin{array}{l}\text { Pre-school } \\
\text { language/s }\end{array}$ \\
\hline $\begin{array}{l}\text { S1 } \\
(\mathrm{M})\end{array}$ & $\mathrm{NZ}$ & $\begin{array}{l}18-25 \\
\text { yrs }\end{array}$ & $\begin{array}{l}\text { Samoan } \\
\text { and English }\end{array}$ & $\begin{array}{l}\text { 75\% Samoan, } \\
25 \% \text { English }\end{array}$ & $\begin{array}{l}90 \% \text { English, } \\
10 \% \text { Samoan }\end{array}$ & English \\
\hline $\begin{array}{l}\text { S4 } \\
(\mathrm{M})\end{array}$ & $\mathrm{NZ}$ & $\begin{array}{l}18- \\
25 y r s\end{array}$ & $\begin{array}{l}\text { Fijian and } \\
\text { English }\end{array}$ & $\begin{array}{l}\text { Mostly Fijian } \\
\text { with } \\
\text { Grandparent, } \\
\text { mixed Fijian } \\
\text { and English } \\
\text { with parents }\end{array}$ & English & $\begin{array}{l}\text { English and } \\
\text { Fijian } \\
\text { (language } \\
\text { nest) }\end{array}$ \\
\hline $\begin{array}{l}\text { S5 } \\
(\mathrm{M})\end{array}$ & $\mathrm{NZ}$ & $\begin{array}{l}18-25 \\
\text { yrs }\end{array}$ & $\begin{array}{l}\text { English and } \\
\text { Niuean }\end{array}$ & $\begin{array}{l}98 \% \text { English, } \\
2 \% \text { Niuean }\end{array}$ & English & $\begin{array}{l}\text { Niuean } \\
\text { immersion } \\
\text { language } \\
\text { nest }\end{array}$ \\
\hline $\begin{array}{l}\text { S7 } \\
(\mathrm{F})\end{array}$ & NZ & $\begin{array}{l}18- \\
25 y r s\end{array}$ & $\begin{array}{l}\text { Samoan } \\
\text { and English }\end{array}$ & $\begin{array}{l}\text { Predominantly } \\
\text { English but at } \\
\text { stages it was } \\
75 \% \text { English, } \\
25 \% \text { Samoan } \\
\text { with } \\
\text { grandparent as } \\
\text { caregiver. }\end{array}$ & English & English \\
\hline $\begin{array}{l}\text { S9 } \\
\text { (F) }\end{array}$ & $\mathrm{NZ}$ & $\begin{array}{l}18- \\
25 y r s\end{array}$ & $\begin{array}{l}80 \% \\
\text { Samoan, } \\
20 \% \\
\text { English }\end{array}$ & Samoan & English & $\begin{array}{l}\text { English and } \\
\text { Samoan } \\
\text { (language } \\
\text { nest) }\end{array}$ \\
\hline $\begin{array}{l}\text { S11 } \\
(\mathrm{F})\end{array}$ & $\mathrm{NZ}$ & $\begin{array}{l}18- \\
25 y r s\end{array}$ & $\begin{array}{l}60 \% \\
\text { English } \\
40 \% \\
\text { Samoan }\end{array}$ & $\begin{array}{l}\text { Samoan and } \\
\text { English }\end{array}$ & & English \\
\hline $\begin{array}{l}\text { S12 } \\
(\mathrm{M})\end{array}$ & $\mathrm{NZ}$ & $\begin{array}{l}18- \\
25 y r s\end{array}$ & $\begin{array}{l}60 \% \\
\text { English } \\
40 \% \\
\text { Samoan }\end{array}$ & $\begin{array}{l}\text { Predominantly } \\
\text { English }\end{array}$ & $\begin{array}{l}\text { English and } \\
\text { some Samoan } \\
\text { (with } \\
\text { extended } \\
\text { family } \\
\text { members) }\end{array}$ & English \\
\hline $\begin{array}{l}\text { S13 } \\
\text { (PF) }\end{array}$ & Samoa & $\begin{array}{l}25+ \\
\mathrm{yrs}\end{array}$ & Samoan & Samoan & Samoan & $\begin{array}{l}\text { No pre- } \\
\text { school }\end{array}$ \\
\hline $\begin{array}{l}\text { S14 } \\
(\mathrm{F})\end{array}$ & NZ & $\begin{array}{l}18-25 \\
\text { yrs }\end{array}$ & $\begin{array}{l}70 \% \text { English } \\
\text { and 30\% } \\
\text { Tokelauan }\end{array}$ & $\begin{array}{l}\text { Parents speak } \\
\text { Tokelauan, } \\
\text { children } \\
\text { answer in } \\
\text { English }\end{array}$ & English & English \\
\hline
\end{tabular}




\begin{tabular}{|c|c|c|c|c|c|c|}
\hline $\begin{array}{l}\text { S15 } \\
(\mathrm{F})\end{array}$ & $\mathrm{NZ}$ & $\begin{array}{l}18-25 \\
\text { yrs }\end{array}$ & $\begin{array}{l}\text { Samoan } \\
\text { and English }\end{array}$ & $\begin{array}{l}\text { Pre-school } \\
90 \% \text { Samoan, } \\
10 \% \text { English, } \\
\text { but later 10\% } \\
\text { Samoan, 90\% } \\
\text { English }\end{array}$ & $\begin{array}{l}\text { Increasing } \\
\text { English as } \\
\text { they grew } \\
\text { older. Now } \\
\text { predominantly } \\
\text { English. }\end{array}$ & \\
\hline $\begin{array}{l}\text { S17 } \\
\text { (PF) }\end{array}$ & Samoa & $\begin{array}{l}18-25 \\
\text { yrs }\end{array}$ & $\begin{array}{l}\text { Samoan } \\
\text { and English }\end{array}$ & $\begin{array}{l}\text { Mostly } \\
\text { Samoan, some } \\
\text { English }\end{array}$ & $\begin{array}{l}\text { Samoan and } \\
\text { English }\end{array}$ & $\begin{array}{l}\text { Went to } \\
\text { English } \\
\text { speaking pre- } \\
\text { school in } \\
\text { Samoa }\end{array}$ \\
\hline $\begin{array}{l}\text { S18 } \\
(\mathrm{PF})\end{array}$ & Tokelau & $\begin{array}{l}18-25 \\
\text { yrs }\end{array}$ & $\begin{array}{l}\text { Tokelauan } \\
\text { and English }\end{array}$ & $\begin{array}{l}90 \% \\
\text { Tokelauan } \\
10 \% \text { English }\end{array}$ & $\begin{array}{l}\text { Tokelauan } \\
\text { and English }\end{array}$ & $\begin{array}{l}\text { Tokelauan } \\
\text { and English } \\
\text { (bilingual } \\
\text { kindergarten) }\end{array}$ \\
\hline $\begin{array}{l}\text { S19 } \\
(\mathrm{M})\end{array}$ & NZ & $\begin{array}{l}18-25 \\
\text { yrs }\end{array}$ & $\begin{array}{l}\text { Tongan and } \\
\text { English }\end{array}$ & $\begin{array}{l}80 \% \text { English } \\
20 \% \text { Tongan }\end{array}$ & English & English \\
\hline $\begin{array}{l}\text { S20 } \\
(\mathrm{M})\end{array}$ & NZ & $\begin{array}{l}18-25 \\
\text { yrs }\end{array}$ & $\begin{array}{l}\text { Samoan } \\
\text { and English }\end{array}$ & $\begin{array}{l}\text { Parents speak } \\
\text { Samoan, } \\
\text { Children } \\
\text { answer in } \\
\text { English }\end{array}$ & English & $\begin{array}{l}\text { Went to } \\
\text { Samoan } \\
\text { language } \\
\text { nest then } \\
\text { moved to } \\
\text { English } \\
\text { speaking } \\
\text { Kindergarten } \\
\text { at 3-4 yrs. }\end{array}$ \\
\hline
\end{tabular}

The 14 Pacific Readers' interview notes were analysed for ideas represented and those found to be repeated in a number of Readers' transcripts were identified as themes. What follows is a presentation of major themes emerging from this qualitative analysis:

\section{Competing interests}

It was apparent that during the years of compulsory education there were many and various academic and recreational 'competing interests' to reading. These included sport, music, dance, church and community activities, cultural performances, boyfriends/girlfriends, socializing, homework, and later cellphones and the internet. Due to the time demands of these activities, the Readers "found it harder to get time to read". Most reported still generally liking reading but simply not having the time to do it in lives that were full and 
varied, especially for those who had shown particular talent in certain areas from an early age and had been heavily involved in them at a competitive level. Cultural and religious expectations and family obligations also require considerable amounts of time investment. The dilemma is how such widely talented students can be empowered to utilize these talents and contribute meaningfully to their socio-cultural contexts without their reading literacy development being negatively affected? According to Edwards and Turner (2009, p.637) one way is that "research and practitioner communities must become more sensitive to this sense of double-consciousness that many minorities hold". They stressed the importance of educators being mindful of the sometimes conflicting desires of students to connect with their own cultural communities while at the same time coming to "know the code of power" in order to be able to succeed in wider society.

\section{"I'd only read if I had to"}

This study has confirmed the critical role of motivation in academic reading. "I've never had a problem with reading, I just didn't do it", "If we had to read it I'd read it. If it wasn't compulsory I wouldn't bother', and similar such statements were found in the majority of the Readers' interview transcripts. This is clear evidence that in their educational history these Pacific student Readers had for various reasons developed a view of academic reading as a (sometimes) 'necessary evil'. The time and effort required was weighed against the perceived necessity of doing it, and the motivation was largely external and negatively framed. In other words, if the negative consequences were perceived to be great enough, one would engage in (or at least provide the appearance of having engaged in) the academic reading activity required in order to avoid the negative consequences. Why this may be the case is an issue requiring investigation. Nist and Simpson (2000, p.651) report that "skill, will, and social support are deemed essential if maximum motivation is 
to occur, but the will component is the centre if a student is to become truly self-regulated." It is important that we come to know more about why reading (an activity that is inherently central to 'learning' in a literate culture where knowledge is stored and transmitted in written form) is so peripheral to these and other students' lives.

\section{Reading of 'popular culture' book series}

An interesting feature of the reading histories of these Pacific Readers was that, even those who professed to have very seldom engaged in recreational reading, popular book series such as Goosebumps, Babysitter's Club, Harry Potter, Horrible Histories, Sweet Valley High, and Hairy Maclairy featured prominently in many of their memories. Why this is so is not known, but possible reasons are: (1) the content of many of these series is of course designed to be particularly interesting to the age groups involved, (2) their mass popularity meant they became part of popular culture and hence a means for social inclusion. Thus, while reading is essentially a solitary and 'antisocial' activity in itself, it may be that the positive peer influence associated with this form of communal reading recaptures the social benefits these readers desired. This certainly deserves further investigation as to how these lessons may be applied in higher education in relation to investment in the communal and social aspects of reading.

\section{Church and Sunday-school reading}

Despite some variation amongst families and age groups, the prominent place of Church as a literacy location in these Pacific Readers' and their family members' lives was clear. It was a venue in which literacy in both English and their family's Pacific language was reinforced, and weekly opportunities for guided and purposeful engagement with specific text types were presented. 
This literacy environment has considerable influence on shaping many of these Pacific Readers' skills sets and beliefs about text. An example of this is the idea of reading as 'performance' emerged in the memories of a number of readers. This is perhaps unsurprising in communities from predominantly oral cultural backgrounds where there is a clear emphasis on oral performance and success in reading often being judged in terms of pronunciation-related aspects. This emphasis on reading as performance lingered in subtle ways in many of the Readers' current conceptions of reading.

\section{Books in the home}

The Pacific Readers varied in their reports of the presence of books in the home. . There were those for whom the quote "There weren't many books around when I was young" holds true, and there were others who reported access to books, for instance, "I didn't have any books of my own but I remember reading my sister's books when she had finished them". A number of Readers recalled books belonging to adults in the home who were teachers or church leaders, or the presence of newspapers or adult work-related reading material. Several recalled their mothers taking them to public libraries and borrowing picture books from there. Overall, however, very few recalled ageappropriate reading material in the home specifically for them.

\section{Reading role models}

Readers' reports varied in relation to the presence of reading role models in the home. For some, the reality was that reading simply did not have a day to day place in the lives of any family members: "I guess we didn't read that much at home". For others, some family members were clearly reading role models, for instance, "Thinking back I'd see her reading a lot", while others were not "Dad probably hasn't read a book in years". Some Readers 
reported reading role models being outside the immediate family, namely aunts or teachers. For some, the memory of people reading in the home was strong, while for others it had not been consciously assimilated, but, looking back, they now realized that the presence of reading role models had had an influence on them.

\section{The influence of teachers}

Teachers played a significant role in reading memories, some positively, for example, "They really encouraged me to read outside my comfort zone", and some negatively, for instance, "I think I kind of lost confidence in my reading". Many could recall specific significant formative experiences with particular teachers, which had had long-lasting impacts on their reading persona, even to the point of being traced to recent experiences in higher education. In research in the US, it was noted by Bean (2000, p.637) that content teachers have a "tremendous influence" on the reading attitudes and enjoyment, engagement and pursuits of students, and this certainly also appeared to be the case with these Pacific Readers.

\section{'Phase changes'}

This relates to changes and periods of transition noticed by Readers in their reading experiences, the texts and reading tasks at school, and their own reading attitudes at certain pivotal points in primary and secondary school. A 'phase change' was noticed upon entering Intermediate School, where reading suddenly seemed to become more "serious". There was also a point at fourth form when Readers reported an increase in the volume and complexity of texts. Entrance to higher education marked another and perhaps the most significant 'phase change', and one for which they receive little systematic assistance from the institution. Bean (2000, p.637) documents the effects of 
such phase changes, noting that "a precipitous decline in attitude toward reading occurred by the middle stage of their school experiences" and that this was largely due to a change in reading material encountered and the experience of content reading as "forced labor". This decline in attitudes was found to have a direct impact on content learning and thought to influence how students approached or avoided future difficult reading tasks.

\section{The importance of Interest}

For the majority of these Pacific Readers, interest appeared to play a considerable role in motivating and sustaining reading. The best example of this is the Reader who reported reading several other challenging and controversial texts after completing the compulsory reading of Animal Farm and 1984 at high school. Those novels "kind of kicked off my interest in government and politics", and the result was apparent even in higher education. This example typifies the power of interest in driving the reading that did occur in the histories of these Readers. Knowing this, the question arises as to how, in the context of higher education and with another genre (academic text), one can ignite and sustain that all-important spark of interest.

\section{Reading as virtue}

Some of the Readers reported being pushed by family to achieve academically. These Readers tended to construe self-imposed reading as an act of 'virtue'. They recalled setting out to read ambitious books "just to make me feel good about myself". Their salient memories included being called up at assembly for prizes for reading/schoolwork, and reading ahead in the textbooks at school in order to "show off" in class. However the downfall of such motivation was that it appeared to be relatively short-lived and the Readers reported seldom actually completing the readings they started or, if 
they did, they were seldom able to repeat the feat or sustain the achievement. These same Readers struggled greatly with motivational issues in their reading in higher education.

When comparing the themes from the reading histories of these Pacific Readers with the findings from other strands within the study it was apparent that the beliefs, experiences and values at the heart of many of these childhood memories of reading had effects that were still being felt in the first year of higher education. Habits, modes of thinking, and ways of responding to texts that had developed throughout compulsory education were still being used and experienced in higher education, often detrimentally. The insights from the Readers' reading histories and backgrounds help in understanding them as readers. So too do their vocabulary sizes and the word power with which they can approach academic texts. This strand is examined next.

\section{(7.2) Vocabulary Size Test Results for First Year Pacific and Other Students}

The Vocabulary Size Test was taken by a total of 38 first year students, including the 12 Pacific case study participants as well as 26 other first year students at the University whose results were collected in order to provide a means of comparison and investigate the existence of a difference between the vocabulary sizes of students of Pacific descent and those from non-Pacific backgrounds within higher education. Of the 26 additional student participants, 17 self identified as speakers of English as a first language and not being of Pacific descent, 5 self identified as being of Pacific descent, and a further 5 self identified as being ESL speakers not of Pacific descent. 


\section{(7.2.1) Vocabulary Size Test Results}

The first interesting finding to emerge from the vocabulary profiling was the considerable variation within the Pacific student group. This is likely to come as little surprise to those working with Pacific students though, since it is simply reflective of the huge ethnic, cultural and linguistic diversity that exists within this group. The results of the 1000 to 14000 levels size test can be found in Appendix 6, as can a bar chart created to visually present the results obtained from the 12 first year Pacific case study student Readers.

The combined average of the 12 case study students' estimated percentage knowledge of each of the levels is shown in Table 7.2 below:

Table 7.2: Pacific student readers' combined average estimated knowledge of the vocabulary levels

\begin{tabular}{|l|l|l|}
\hline $\begin{array}{l}\text { Vocabulary } \\
\text { Level }\end{array}$ & $\begin{array}{l}\text { Combined average } \\
\text { estimated number of } \\
\text { words known at this } \\
\text { level (Averaged across } \\
\text { all case study Readers) }\end{array}$ & $\begin{array}{l}\text { Pacific case study } \\
\text { students' combined } \\
\text { average estimated } \\
\text { percentage knowledge of } \\
\text { the level (\%) }\end{array}$ \\
\hline 1000 & 950 & $95 \%$ \\
\hline 2000 & 970 & $97 \%$ \\
\hline 3000 & 920 & $92 \%$ \\
\hline 4000 & 800 & $80 \%$ \\
\hline 5000 & 960 & $96 \%$ \\
\hline 6000 & 710 & $71 \%$ \\
\hline 7000 & 610 & $61 \%$ \\
\hline 8000 & 770 & $77 \%$ \\
\hline 9000 & 500 & $50 \%$ \\
\hline 10000 & 660 & $66 \%$ \\
\hline 11000 & 560 & $56 \%$ \\
\hline 12000 & 680 & $68 \%$ \\
\hline 13000 & 350 & $35 \%$ \\
\hline 14000 & 390 & $39 \%$ \\
\hline
\end{tabular}


Readers' combined average estimated knowledge of the Levels is shown in Figure 7.1.

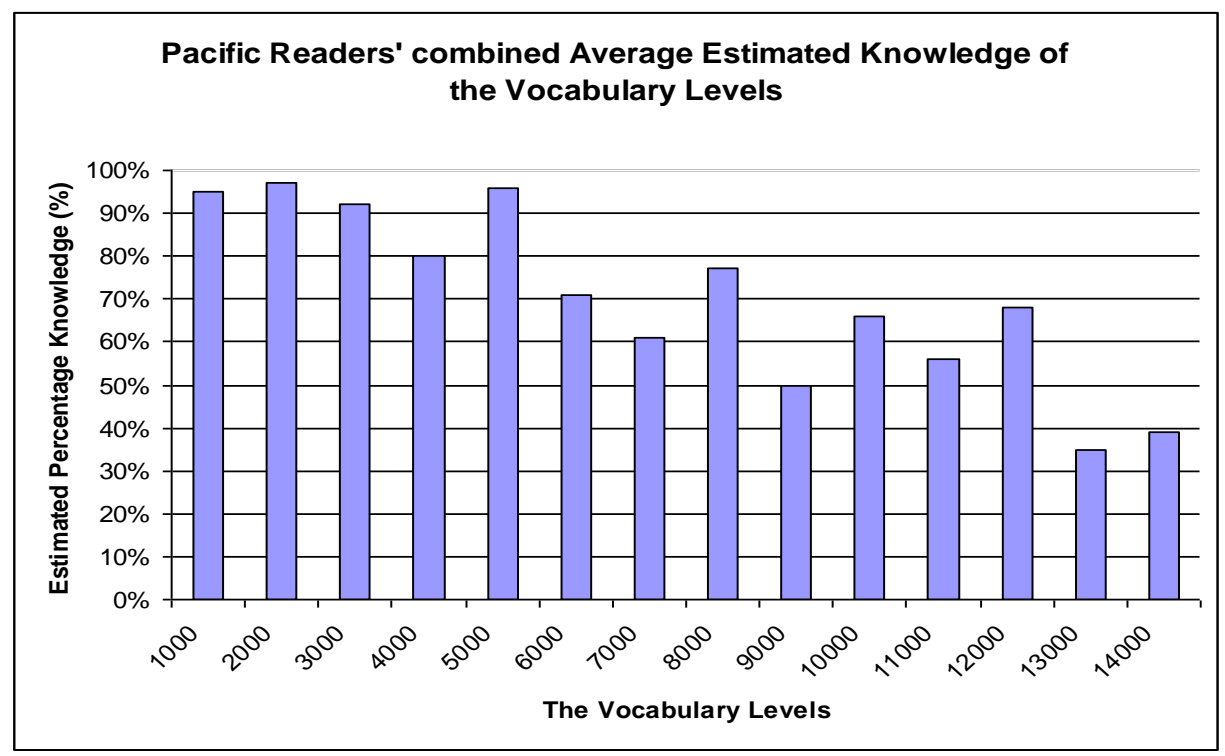

Figure 7.1: Pacific Readers' combined average estimated knoweldge of the vocabulary levels K1-K14

Overall, the gradual decline in percentage knowledge as the vocabulary levels increase is expected since the words in these upper levels represent words of decreasing frequency and thus, the likelihood of Pacific students knowing them also decreases - as it would be likely to with any student population. Interestingly, however, the rate of decline in knowledge did appear to differ by ethnic and first language background, but discussion of this is not within the scope of this thesis.

A second observation relates to the average estimated knowledge of levels K1 (the first 1000) and K2 (the second 1000, or 2000 level). The combined average estimated knowledge of these levels was not 100\% (being 95\% and 97\% respectively), suggesting that gaps still exist in knowledge of general 
vocabulary. Individual variation did of course exist and this is apparent in the individual Reader Profiles.

\section{(7.2.2) Academic Word Test Results}

In addition to the Vocabulary Size Test, a smaller measure was also taken using a 30 word sample from the University Word List (Xue \& Nation, 1984). This Academic Vocabulary test is part of Schmitt, Schmitt and Clapham's Vocabulary Levels Test 5 (Version 2) (2001). Although these academic items can be found within the 1st 1000 to the 10th 1000 of the Vocabulary Size Test (Nation \& Beglar, 2007, p.2), the Academic Vocabulary measure provides a specific indication of the students' academic word knowledge.

The overall average estimated percentage knowledge of the UWL for all the Pacific students involved was $88 \%$. The Readers' results are shown in Figure 7.2. 


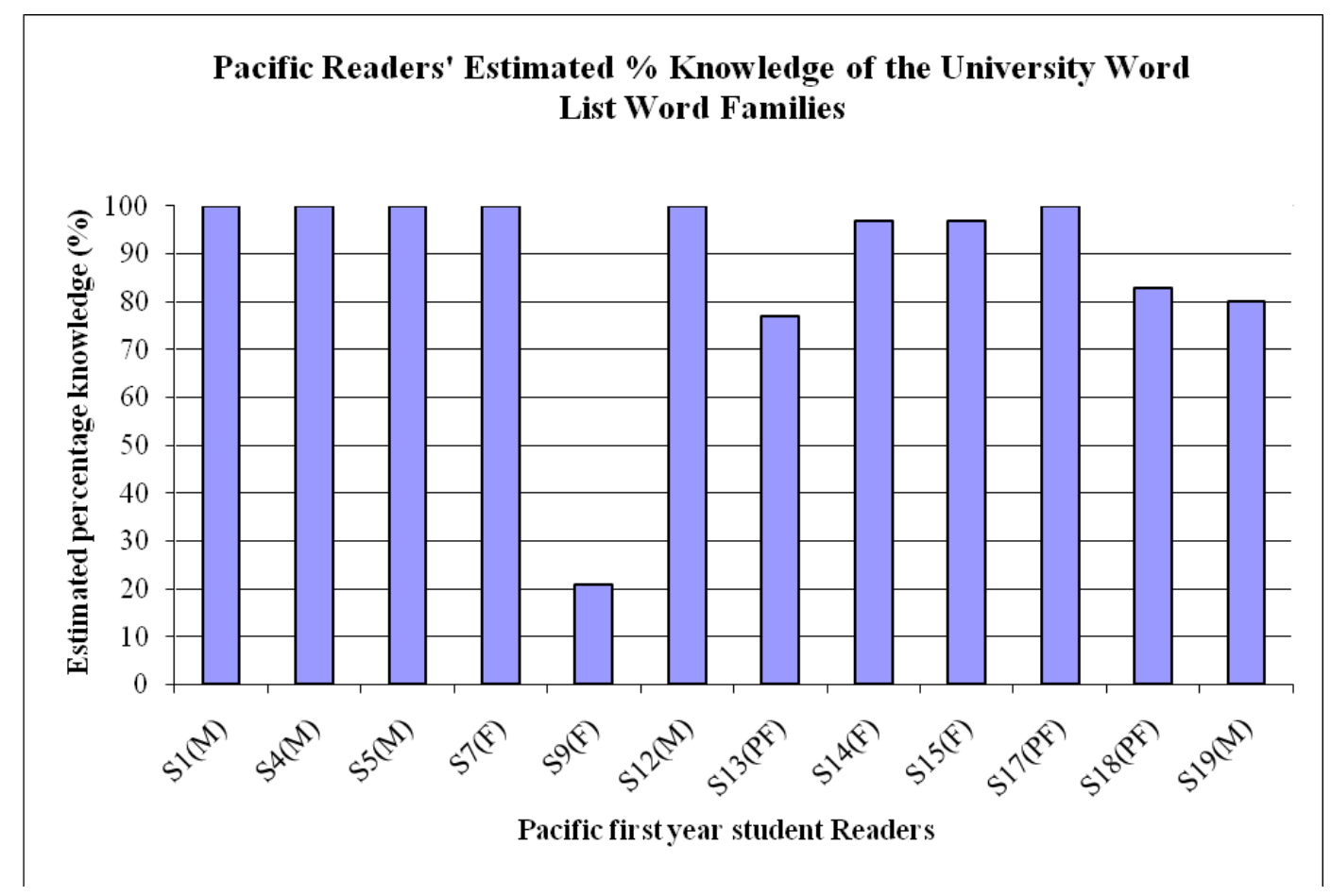

Figure 7.2: Pacific Readers' estimated percentage knowledge of the University Word List

Interestingly, the results of the UWL sample measure revealed even greater variation within this Pacific group than the general vocabulary size test. This diversity has implications for the development of any method that seeks to address these vocabulary shortfalls amongst this student group.

Results also showed that a third of the case study Readers (S9(F), S13(PF), S18(PF) and S19(M)) could be expected to possess relatively limited receptive knowledge of the academic vocabulary items in the University Word List, having scored less than $85 \%$ in the test. Since these UWL items are, by their very nature, known to occur with frequency in academic text this limitation is of some immediate concern and would merit developmental attention for these Readers. 
Of the four lower scoring Readers, two (S13(PF) and S18(PF)) were born and received their early education in a Pacific Nation before emigrating to New Zealand either as a young adult or child with their families, and the other two (S9(F) and S19(M)) although New Zealand born, came from homes in which the dominant language during childhood was the Pacific mother tongue of their parents (and still is today in one case). This leads fairly inevitably to more questions about the influence of the home language environment on English language vocabulary development. These are beyond the scope of this research but of considerable importance to reading and future research in New Zealand.

The high estimated knowledge of academic vocabulary that the other eight Readers' results indicate is an encouraging result, and suggests that while there is some variation, these Pacific Readers had good knoweldge of the tyes of vocabulary that are known occur frequently in academic texts from a variety of different disciplines.

\section{(7.2.3) How do Pacific Student Readers' Vocabulary Sizes Compare to their Non-Pacific Peers?}

The Vocabulary Size Test was the only measure for which a direct comparison was made between these Pacific Readers and their non-Pacific peers. For the purposes of comparison, two groups were identified: (1) 16 'Pacific', composed of the 12 case study students and 4 other Pacific-descent first year student participants, and (2) 17 non-Pacific ('Non-P'), composed of first year students who self identified as first language speakers of English and not being of Pacific descent. A statistical T-test was carried out to ascertain whether a statistically significant difference existed between the vocabulary size test results of the Pacific and the non-Pacific participants. The results are shown in Table 7.3 below. 
Table 7.3: T-test results for comparison of vocabulary levels test results of Pacific and non-Pacific first year students

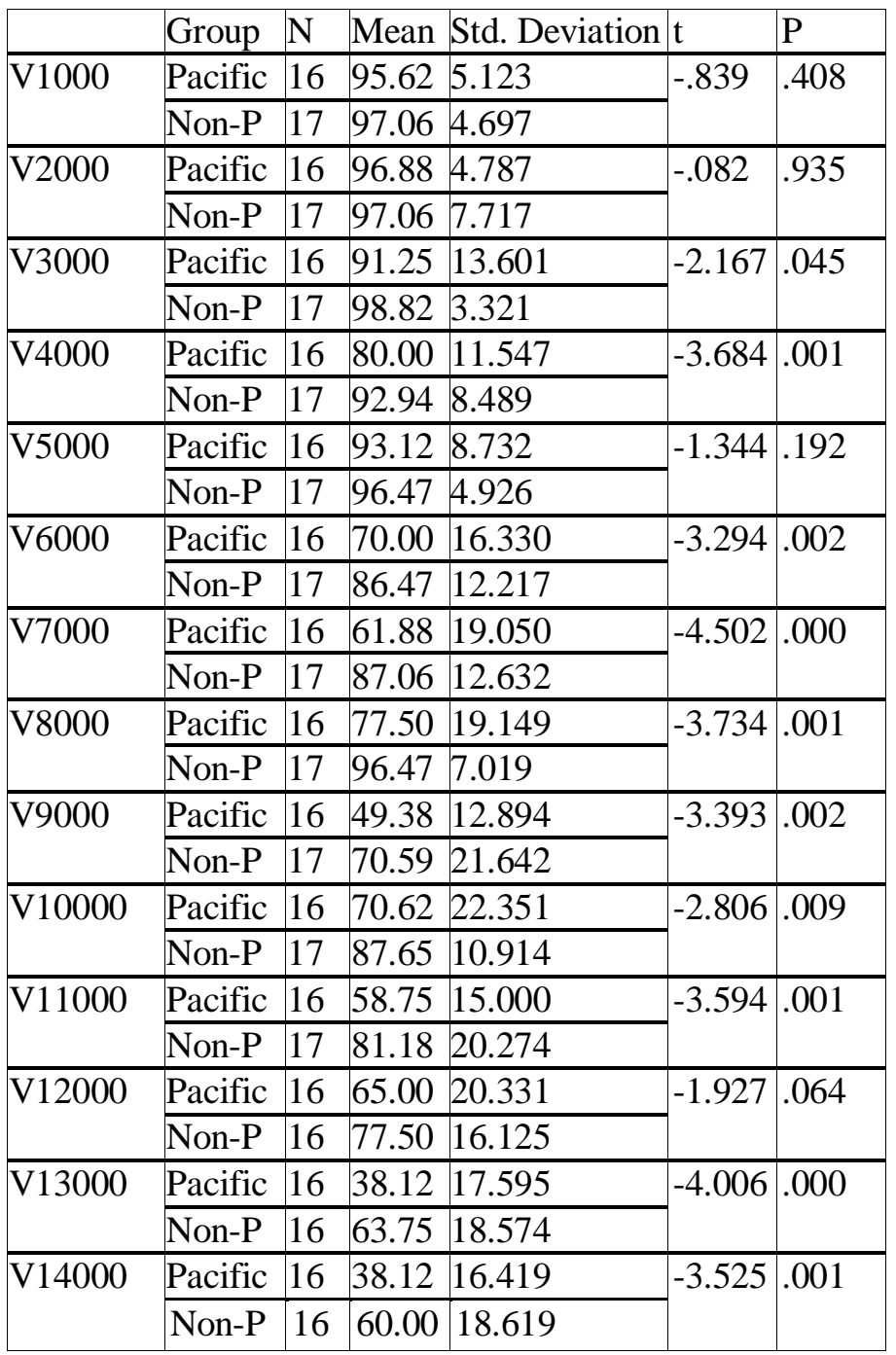

The results clearly indicated that the averages of the non-Pacific students' vocabulary sizes were larger than the averages of the Pacific students' vocabulary sizes at all levels of the test. This difference was statistically significant for all but four of the 14 vocabulary levels tested, and in several cases the difference was highly significant. The four levels not indicating a 
statistical difference were the 1000 and 2000 (K1 and K2), as well as the 5000 (K5) and the 12000 (K12) levels. However the difference between the two groups at the $\mathrm{K} 12$ level is very nearly significant, and the K5 result may possibly be the result of an anomaly of the test itself, the particular items selected, or due to societal factors.

The closeness of the two groups in the $\mathrm{K} 1$ and $\mathrm{K} 2$ levels shows that it is only really from $\mathrm{K} 3$ onwards that the considerable divergence in the vocabulary knowledge of the Pacific and non-Pacific students actually begins. A suggested explanation for this is that the first two levels ( $\mathrm{K} 1$ and $\mathrm{K} 2$ ), being the two most frequent, are those which are used most often for daily communication, and are acquired primarily in this manner. As such, both Pacific and non-Pacific scores show no significant difference at these levels. However from K3 onwards, acquisition cannot usually occur only from conversational sources (since most people do not use these words in daily conversation) and it is this higher level at which there is a clear deviation between the Pacific and non-Pacific groups in terms of vocabulary knowledge.

The results show that these Pacific students entered University with a significantly smaller English language vocabulary size than their non-Pacific peers. This is of concern because the majority of these Pacific Readers were in fact New Zealand born and raised and educated, often reportedly speaking English as a 'first' language, attending the same schools and ostensibly being provided the 'same' educational opprtunities as their non-Pacific native English speaking peers. Yet, as this year's cohort enters higher education, the two groups do so on clearly different footings. 


\section{(7.2.4) The Pacific Student Readers' Vocabulary Size Estimates \& Their Performance on the Free Recall Task}

Of interest having ascertained the previous worrying results, is how Readers' vocabulary sizes relate to their actual success in the recall of text. For this purpose the relationship between four of the Course A Readers' overall estimated Vocabulary sizes (from K1-K14 Vocabulary Size test) and their success in Recall (\% IU Recall) has been investigated (these four were the only ones who returned sufficiently complete data to make the comparison). These results are shown in Figure 7.3 below.

Figure 7.3: Selected Course A Pacific students' estimated knowledge of vocabulary levels 1-14 \& average percentage IU recall from Course A texts

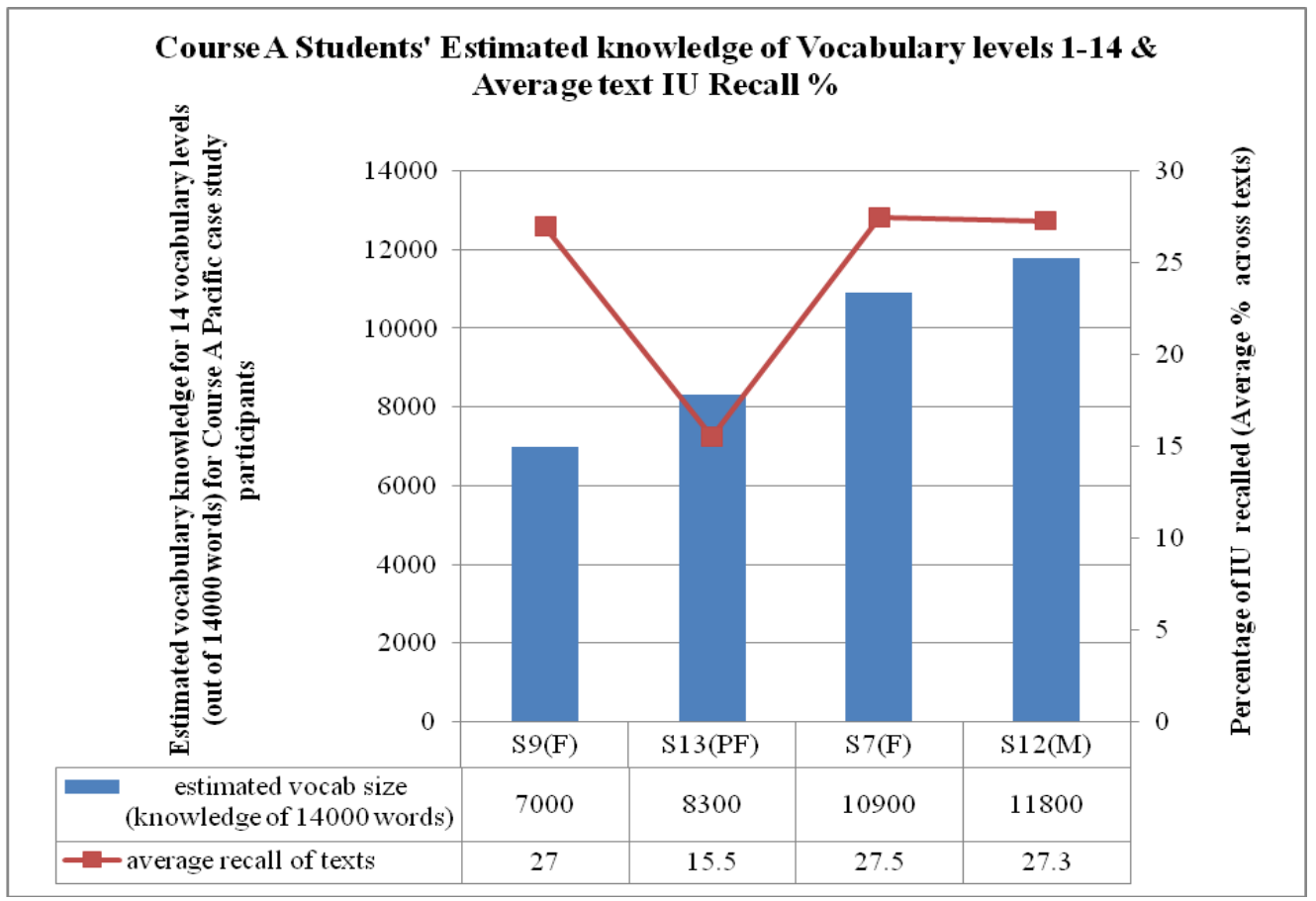

There being only four readers involved means that the results are not widely generalisable. However, the chart still reveals some interesting results. Of 
particular note is the observation that Reader $\mathrm{S} 9(\mathrm{~F})$, with the smallest estimates of vocabulary size, scored the same overall average \% IU Recall across 6 texts as those with the highest vocabulary sizes.

From S9(F)'s results it can be inferred that there are other mitigating factors (such as effective strategy use, metacognitive control or affective response) that enables Recall to be as high for those with smaller vocabulary sizes as it does for those with comparably large vocabularies. This result appears to be sympathetic to the compensatory processing model of reading, where readers' strengths compensate for their weaknesses in a dynamic manner during reading, and "a deficit in any particular process will result in a greater reliance on other knowledge sources" (Stanovich, 1980, p.32).

The vocabulary situation of these Readers is clearly a complex one. Had S9(F) and S13(PF) been judged solely on the outcome of their vocabulary tests, it would not traditionally have been thought that they could read as independently or successfully as their peers with larger vocabularies (S7(F) and S12(M)). This prediction would have been accurate for S13(PF), but clearly inaccurate for $S 9(F)$. This finding can be viewed as positive in the sense that it provides evidence that those with smaller vocabulary sizes, such as $\mathrm{S} 9(\mathrm{~F})$, may be enabled to understand and remember as well as those with larger vocabularies if other mitigating factors can be successfully exploited. However, it does raise definite questions about the use of vocabulary measures alone as a good indicator of potential academic reading ability, thus reducing their value somewhat as a relatively easily obtained predictive measure.

So, how do the vocabulary results in this study compare with other findings? In reporting a study of English second language speaking Omani students' experiences in higher education Cobb and Horst (2001, p.321) reported 
lecturers' and researchers' perceptions that " $[t]$ he main source of the problem appeared to be the students' lack of vocabulary", and moreover, that vocabulary 'shortages' were not only at the technical level but also the general vocabulary levels.

Research on Pacific students and English vocabulary knowledge is scarce, however one such study is that of Marshall and Gilmour (1993) who researched lexical knowledge and reading comprehension in Papua New Guinea. They concluded that PNG students from a wide range of academic levels experienced difficulties in reading due to limitations in their lexical knowledge. Marshall and Gilmour particularly stressed the need for not only the development of technical vocabulary for specific disciplines but also what they termed 'subtechnical' vocabulary. Their definition of subtechnical is "context-independent words which occur with high frequency across disciplines" (1993, p.71) and this appears to correspond very much with what has since been termed 'academic vocabulary' and formalized firstly in the UWL (Xue \& Nation, 1984) and later by Coxhead (2000) in the Academic Word List. They argue that it is the subtechnical vocabulary that are used to "modify or to express the relations that exist between the key concepts of the discipline" (1993, p.71).

It is suggested that knowledge of the $\mathrm{K} 1$ and $\mathrm{K} 2$ levels may be acquired largely through hearing these items in use and acquiring receptive and productive knowledge of these vocabulary items in the natural manner of conversation. However the Academic Vocabulary and the mid and upper of the vocabulary levels are seldom able to be obtained from conversation, particularly in homes where English is not the language of the parents/caregivers or other primary language conversants. Where then can such necessary vocabulary be acquired? Evidence suggests that this language 
must be primarily acquired from books, through extensive and intensive reading (Carrell \& Carson 1997). It is strongly suggested that the type of regular, extensive and intensive reading experiences with text of appropriate levels of difficulty, which is interest-driven and therefore enjoyed, is that which fosters vocabulary growth in the language of choice.

Can it then be supposed therefore that the Pacific participants in this study whose vocabulary measures indicated limited vocabulary size and breadth were this way primarily as a result of not having been exposed to significant enough amounts of these and other similar types of reading experiences? Perhaps it partially can. Mangubhai (1995, p.15) argues that

[b]oth reading and writing have served a limited purpose for Pacific societies. From this it cannot be inferred that reading and writing are not regarded as important by parents for their children. Children are told both by their parents and their teachers about the importance of reading and writing in the school contexts. However, the rhetoric is not matched by corresponding encouragement or social approval for school-based activities to spill over into the society.

However, it may not be sufficient to say that the provision of such reading experiences is sufficient to build an extensive vocabulary. This is particularly evidenced once again in Reader S9(F), whose reading history reveals frequent and relatively extensive reading throughout most of her compulsory schooling years, yet who scores lowest on the traditional measures of vocabulary in this study.

What is clear though, overall, is that the family, community and social literacy practices that were valued and participated in by these Pacific Reader participants (predominantly within a New Zealand context) had by various 237 
means equipped them with vocabulary profiles that differed significantly from their non-Pacific peers. Moreover, they possessed the types of vocabulary profiles that may, according to research, result in them facing challenges when dealing with the new types of texts, discourses and reading behaviours valued and practiced within the University.

The findings of this study highlighted that these Pacific student readers, despite being predominantly born, raised and educated in New Zealand in English medium schooling, did not have $100 \%$ knowledge of the general vocabulary in Levels K1-K3. The need to fill these vocabulary gaps presents a unique problem in the first year of university, where the learning curve for content, schedules, routines, and technical vocabulary is so steep that few attentional resources are available for the learning of 'extra' vocabulary. At the same time many readers face what Coady (1997) refers to as the "beginner's paradox" - that reading is needed to develop vocabulary but at the same time, vocabulary knowledge is needed to read. This resembles Pinker's 'learnability paradox' and is a very real issue for Pacific readers for whom even a dictionary entry which is supposed to provide them with the meaning of an unknown word, only further baffles them with more unknown vocabulary (Davidson-Toumu'a, 2005).

\section{(7.3) Reading Rate Measurements of First Year Pacific Students}

Having established that vocabulary is an important area for further consideration, attention is turned now to Reading Rate as a manifestation of reading fluency. In all, a total of 12 Pacific students participated in the Reading Rate measurements for up to 11 authentic text samples each from the 6 first year Humanities and Commerce courses that they represented (Courses A, B, C, D, E, \& F). Below, in Table 7.4, are the Reading Rates of the Pacific 
first year students from whom complete, or almost complete, data samples were able to be obtained in this study:

Table 7.4: Recorded reading rates in words per minute (wpm) of Pacific first year students reading authentic text samples from Courses A, B, C, D, E and $\mathrm{F}$

\begin{tabular}{|c|c|c|c|c|c|c|c|c|c|c|c|c|}
\hline & \multicolumn{11}{|c|}{ Course A Text Samples } & \multirow{2}{*}{$\begin{array}{l}\text { Average } \\
\text { reading } \\
\text { rate for } \\
\text { student }\end{array}$} \\
\hline & A1 & $\mathrm{A} 2$ & A3 & A4 & A5 & A6 & A7 & A8 & A9 & A10 & A11 & \\
\hline S9(F) & 263 & 283 & 261 & 249 & 228 & 231 & 251 & 227 & 274 & 242 & 228 & 249 wpm \\
\hline S4(F) & 163 & 109 & 114 & 120 & 148 & 123 & 105 & 150 & 190 & 118 & 81 & 129 wpm \\
\hline S5(M) & 150 & 100 & 86 & 108 & 99 & 125 & - & - & - & - & - & 111 wpm \\
\hline S7(F) & 270 & 204 & 223 & 207 & 175 & 147 & 173 & 170 & 180 & 203 & 197 & 195 wpm \\
\hline S12(M) & 211 & 196 & 178 & 166 & 199 & 179 & 167 & 117 & 120 & 161 & 204 & 173 wpm \\
\hline $\mathrm{S} 13(\mathrm{PF})$ & 175 & 137 & 133 & 187 & 164 & 156 & 171 & 124 & 199 & 194 & 169 & 164 wpm \\
\hline $\begin{array}{l}\text { AVER- } \\
\text { AGE } \\
\text { Rate for } \\
\text { text } \\
\text { sample }\end{array}$ & $\begin{array}{l}205 \\
\text { wpm }\end{array}$ & $\begin{array}{l}172 \\
\text { wpm }\end{array}$ & $\begin{array}{l}166 \\
\text { wpm }\end{array}$ & $\begin{array}{l}173 \\
\text { wpm }\end{array}$ & $\begin{array}{l}169 \\
\text { wpm }\end{array}$ & $\begin{array}{l}160 \\
\text { wpm }\end{array}$ & $\begin{array}{l}173 \\
\text { wpm }\end{array}$ & $\begin{array}{l}158 \\
\text { wpm }\end{array}$ & $\begin{array}{l}193 \\
\text { wpm }\end{array}$ & $\begin{array}{l}184 \\
\text { wpm }\end{array}$ & $\begin{array}{l}176 \\
\text { wpm }\end{array}$ & \\
\hline
\end{tabular}

\begin{tabular}{|l|l|l|l|l|l|l|l|l|l|l|}
\hline & \multicolumn{1}{|l|}{ Course B Text Samples } & Average \\
& B1 & B2 & B3 & B4 & B5 & B6 & B7 & B8 & B9 $\begin{array}{l}\text { reading } \\
\text { rate for } \\
\text { student }\end{array}$ \\
\hline S14(F) & 81 & 77 & 83 & 80 & 85 & 73 & 106 & 86 & 91 & $85 \mathrm{wpm}$ \\
\hline S15(F) & 75 & 68 & 82 & 66 & 103 & 95 & 121 & 115 & 119 & $94 \mathrm{wpm}$ \\
\hline $\begin{array}{l}\text { AVERAGE } \\
\text { rate for text } \\
\text { sample }\end{array}$ & $\begin{array}{l}78 \\
\text { wpm }\end{array}$ & $\begin{array}{l}73 \\
\text { wpm }\end{array}$ & $\begin{array}{l}83 \\
\text { wpm }\end{array}$ & $\begin{array}{l}73 \\
\text { wpm }\end{array}$ & $\begin{array}{l}94 \\
\text { wpm }\end{array}$ & $\begin{array}{l}84 \\
\text { wpm }\end{array}$ & $\begin{array}{l}114 \\
\text { wpm }\end{array}$ & $\begin{array}{l}101 \\
\text { wpm }\end{array}$ & $\begin{array}{l}105 \\
\text { wpm }\end{array}$ & \\
\hline
\end{tabular}

\begin{tabular}{|l|l|l|l|l|l|l|}
\hline & \multicolumn{4}{|l|}{ Course C Text Samples } & $\begin{array}{l}\text { Average } \\
\text { reading } \\
\text { rate for } \\
\text { student }\end{array}$ \\
\hline C1 & C2 & C3 & C4 & C5 & $\begin{array}{l}121 \\
\text { wpm }\end{array}$ \\
\hline S1(M) & 138 & 129 & 124 & 109 & 107 & \\
\hline $\begin{array}{l}\text { AVERAGE } \\
\text { rate for text } \\
\text { sample }\end{array}$ & $\begin{array}{l}138 \\
\text { wpm }\end{array}$ & $\begin{array}{l}129 \\
\text { wpm }\end{array}$ & $\begin{array}{l}124 \\
\text { wpm }\end{array}$ & $\begin{array}{l}109 \\
\text { wpm }\end{array}$ & $\begin{array}{l}107 \\
\text { wpm }\end{array}$ & \\
\hline
\end{tabular}




\begin{tabular}{|l|l|l|l|l|l|l|l|l|}
\hline & \multicolumn{6}{|l|}{ Course D Text Samples } & $\begin{array}{l}\text { Average } \\
\text { reading } \\
\text { rate for } \\
\text { student }\end{array}$ \\
\hline D1 & D2 & D3 & D4 & D5 & D6 & D7 & $\begin{array}{l}171 \\
\text { wpm }\end{array}$ \\
\hline $\begin{array}{l}\text { S18(PF) } \\
\text { AVERAGE } \\
\text { rate for text } \\
\text { sample }\end{array}$ & 207 & 174 & 216 & 165 & 190 & 145 & 102 \\
wpm & $\begin{array}{l}174 \\
\text { wpm }\end{array}$ & $\begin{array}{l}216 \\
\text { wpm }\end{array}$ & $\begin{array}{l}165 \\
\text { wpm }\end{array}$ & $\begin{array}{l}190 \\
\text { wpm }\end{array}$ & $\begin{array}{l}145 \\
\text { wpm }\end{array}$ & $\begin{array}{l}102 \\
\text { wpm }\end{array}$ & \\
\hline
\end{tabular}

\begin{tabular}{|c|c|c|c|c|c|c|c|c|c|}
\hline & \multicolumn{8}{|c|}{ Course E Text Samples } & \multirow{2}{*}{$\begin{array}{l}\text { Average } \\
\text { reading } \\
\text { rate for } \\
\text { student }\end{array}$} \\
\hline & E1 & E2 & E3 & $\mathrm{E} 4$ & E5 & E6 & E7 & E8 & \\
\hline $\mathrm{S} 17(\mathrm{PF})$ & 113 & 153 & 139 & 88 & 86 & 118 & 83 & 170 & $119 \mathrm{wpm}$ \\
\hline $\begin{array}{l}\text { AVERAGE } \\
\text { rate for text } \\
\text { sample }\end{array}$ & $\begin{array}{l}113 \\
\text { wpm }\end{array}$ & $\begin{array}{l}153 \\
\text { wpm }\end{array}$ & $\begin{array}{l}139 \\
\text { wpm }\end{array}$ & $\begin{array}{l}88 \\
\text { wpm }\end{array}$ & $\begin{array}{l}86 \\
\text { wpm }\end{array}$ & $\begin{array}{l}118 \\
\text { wpm }\end{array}$ & $\begin{array}{l}83 \\
\text { wpm }\end{array}$ & $\begin{array}{l}170 \\
\text { wpm }\end{array}$ & \\
\hline
\end{tabular}

\begin{tabular}{|c|c|c|c|c|c|}
\hline & \multicolumn{4}{|c|}{ Course F Text Samples } & \multirow{2}{*}{$\begin{array}{l}\text { Average } \\
\text { reading } \\
\text { rate for } \\
\text { student }\end{array}$} \\
\hline & F1 & $\mathrm{F} 2$ & F3 & $\mathrm{F} 4$ & \\
\hline S19(M) & 134 & 101 & 112 & 113 & 115 wpm \\
\hline $\begin{array}{l}\text { AVERAGE } \\
\text { rate for text } \\
\text { sample }\end{array}$ & $\begin{array}{l}134 \\
\text { wpm }\end{array}$ & $\begin{array}{l}101 \\
\text { wpm }\end{array}$ & $\begin{array}{l}112 \\
\text { wpm }\end{array}$ & $\begin{array}{l}113 \\
\text { wpm }\end{array}$ & \\
\hline
\end{tabular}

Variation is clear amongst these Pacific Readers, and the Rate at which the Pacific Readers were able to read for the purpose of understanding and remembering academic texts clearly varied from discipline to discipline.

For the purpose of comparison with literature I will focus on Course A. The overall average Reading Rate for Course A Readers in 'reading to understand and remember' was $175 \mathrm{wpm}$. This is somewhat faster than the $138 \mathrm{wpm}$ for students reading to memorise that Carver (1990) identified, however it is not possible to compare the degree of 'success' or 'accuracy' across the two 
studies to make any meaningful comparison of these two Rates while bearing in mind the speed-accuracy trade-off.

Meyer, Talbot and Florencio (1999, p.305) provided a summary of research on Reading Rate in higher education and the average rate at which college/university students read for "concerted long term-memory storage" was found to be between 136-200 wpm - a rate comparable with the results of this study. Carver (1990) observed that there was a relationship between reading rate and reading purpose, and his findings ranged from 300wpm for reading for the purpose of 'understanding', to $200 \mathrm{wpm}$ for reading for the purpose of 'learning' the information, to $138 \mathrm{wpm}$ for the purpose of being able to 'recall' the information from the text. The importance of purpose to Reading Rate cannot therefore be underestimated.

\section{(7.4) Reported Academic Reading Strategies/Habits/Attitudes of First Year Pacific Students}

In the Reading Strategies, Habits and Attitudes Inventory constructed for this study the items are categorized either (1) positively framed (meaning that the majority of the literature surveyed considers these strategies to be those employed often by 'Good Readers') or (2) negatively framed (meaning that research literature often represents these as strategies employed by 'Poor Readers'). Ideally it would be advantageous for students to show higher scores on the positively framed items and low scores on the negatively framed items within the various strategy and attitude categories into which the inventory items have been classified. The overall patterns are presented in the following sections. 


\section{(7.4.1) Overall Inventory Rating Patterns}

As described in the Methodology, a seven point scale was used for student Readers' self-rating. Below in Table 7.11 and Figure 7.4 are the Readers' average ratings on the Inventory categories for the positively framed items. Five of the six Course A participants completed the Inventory, however S5(M) did not due to cultural and community commitments at the time of data collection, and thus is not included in the table below.

Table 7.5: Course A readers' average self-ratings for positively framed inventory items

\begin{tabular}{|c|c|c|c|c|c|c|c|c|c|}
\hline & 宽 & 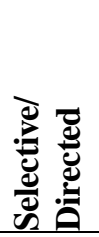 & 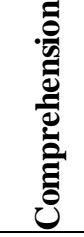 & 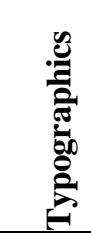 & 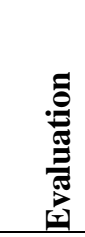 & 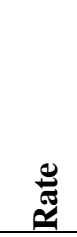 & 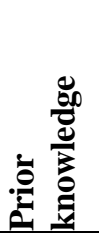 & 离 & 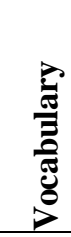 \\
\hline $\begin{array}{l}\text { The Pacific } \\
\text { participant group's } \\
\text { average rating }\end{array}$ & 4.89 & 4.45 & 4.83 & 5.42 & 4.29 & 5.14 & 5.24 & 3.87 & 5.04 \\
\hline $\begin{array}{l}\text { Reader S9(F) } \\
\text { Average rating for } \\
\text { category }\end{array}$ & 5.2 & 4.61 & 4.17 & 4.33 & 4 & 5.8 & 5 & 3.33 & 4.5 \\
\hline $\begin{array}{l}\text { Reader } \mathrm{S} 4(\mathrm{M}) \\
\text { Average rating for } \\
\text { category }\end{array}$ & 5 & 4.07 & 4.47 & 4.66 & 4.17 & 4.6 & 5.66 & 4 & 5 \\
\hline $\begin{array}{l}\text { Reader } \mathrm{S7}(\mathrm{F}) \\
\text { Average rating for } \\
\text { category }\end{array}$ & 4.4 & 5.15 & 4.58 & 7 & 4.28 & 5.2 & 4 & 3 & 4.5 \\
\hline $\begin{array}{l}\text { Reader S12(M) } \\
\text { Average rating for } \\
\text { category }\end{array}$ & 5.2 & 4.37 & 4.37 & 6.66 & 5.57 & 4.6 & 6 & 4.66 & 4 \\
\hline $\begin{array}{l}\text { Reader } \mathrm{S13}(\mathrm{PF}) \\
\text { Average rating for } \\
\text { category }\end{array}$ & 6.2 & 4 & 5.47 & 4.33 & 4.28 & 5.6 & 5.66 & 4.33 & 6 \\
\hline
\end{tabular}




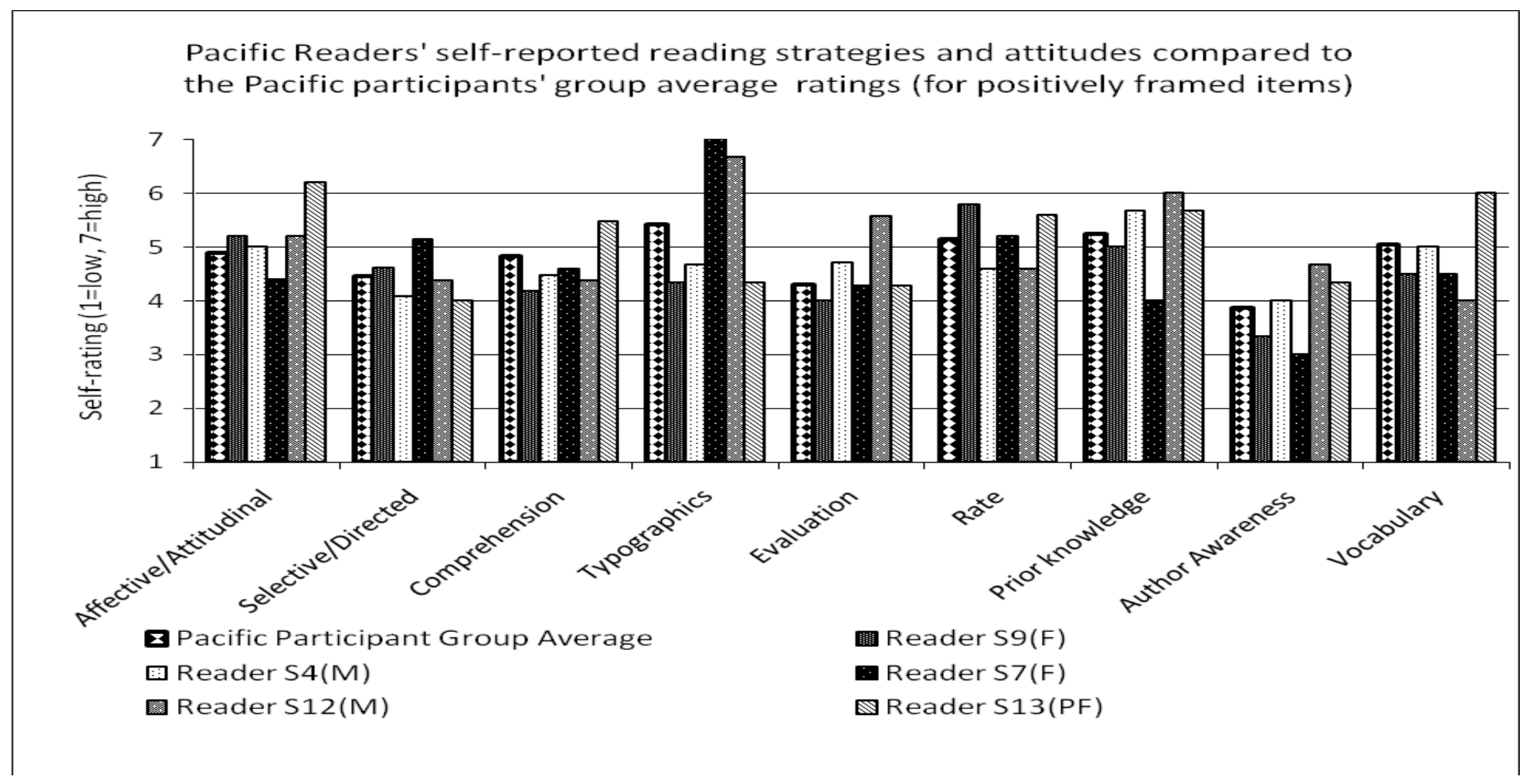

Figure 7.4: Pacific readers' self-reported use of positively framed reading strategies compared to the group average for the Pacific participant group. 
Table 7.6 and Figure 7.5 below show the results for the negatively framed items.

Table 7.6: Course A readers' average self-ratings for negatively framed inventory items

\begin{tabular}{|c|c|c|c|c|c|}
\hline & 焉 & $\begin{array}{l}0 \\
0\end{array}$ & 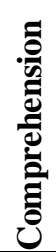 & 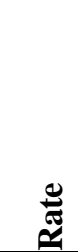 & 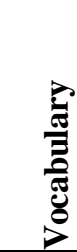 \\
\hline Pacific Participants' Group Average & 4.9 & 4.18 & 5 & 5.39 & 4.09 \\
\hline Reader S9(F) & 5.25 & 4 & 6 & 5 & 3.66 \\
\hline Reader S4(M) & 3.5 & 3 & 4 & 4 & 5 \\
\hline Reader S7(F) & 4.75 & 3 & 7 & 6.25 & 2.33 \\
\hline Reader S12(M) & 3.25 & 2 & 3 & 2.5 & 4 \\
\hline Reader S13(PF) & 4.33 & 4 & 5 & 7 & 6.33 \\
\hline
\end{tabular}

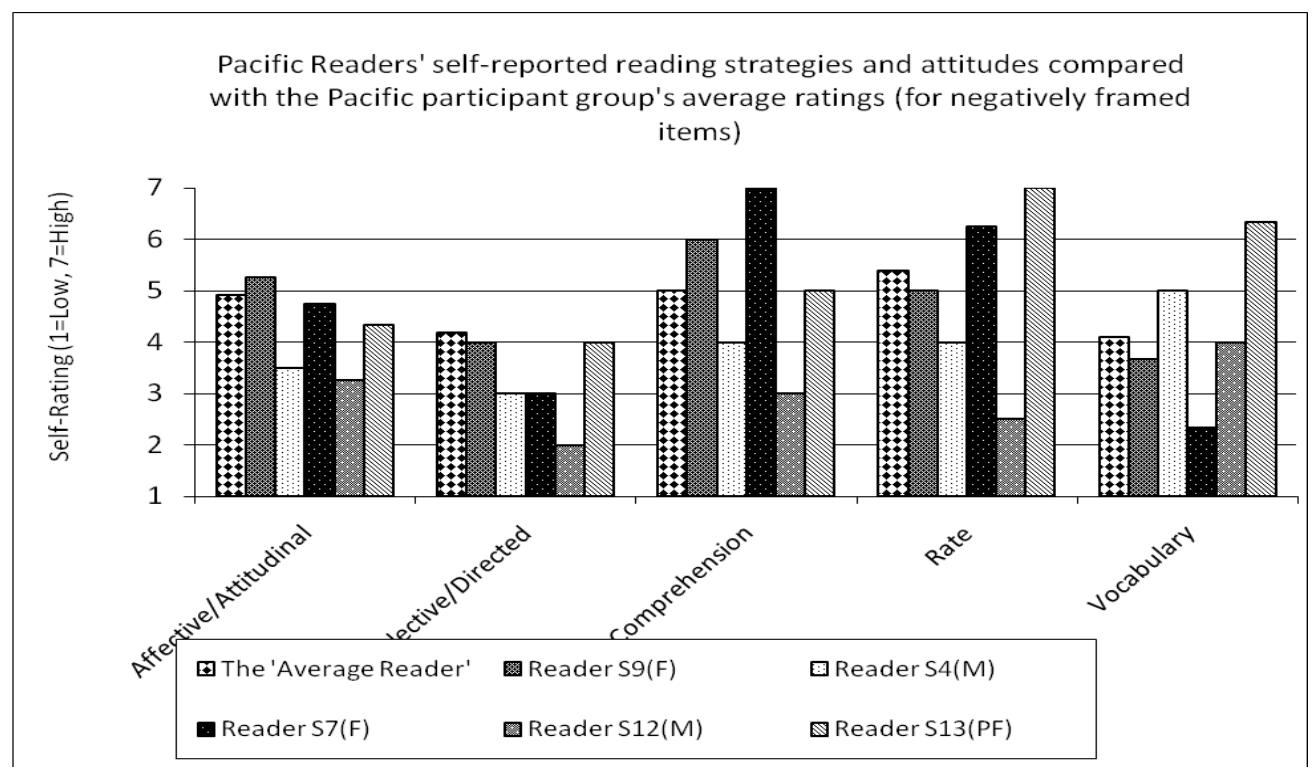

Figure 7.5: Pacific readers' self-reported use of negatively framed reading strategies compared to the average for the Pacific participant group.

The following charts (Figures 7.6 and 7.7) illustrate the differences apparent in the profiles of the various Readers for positively and negatively framed items. 


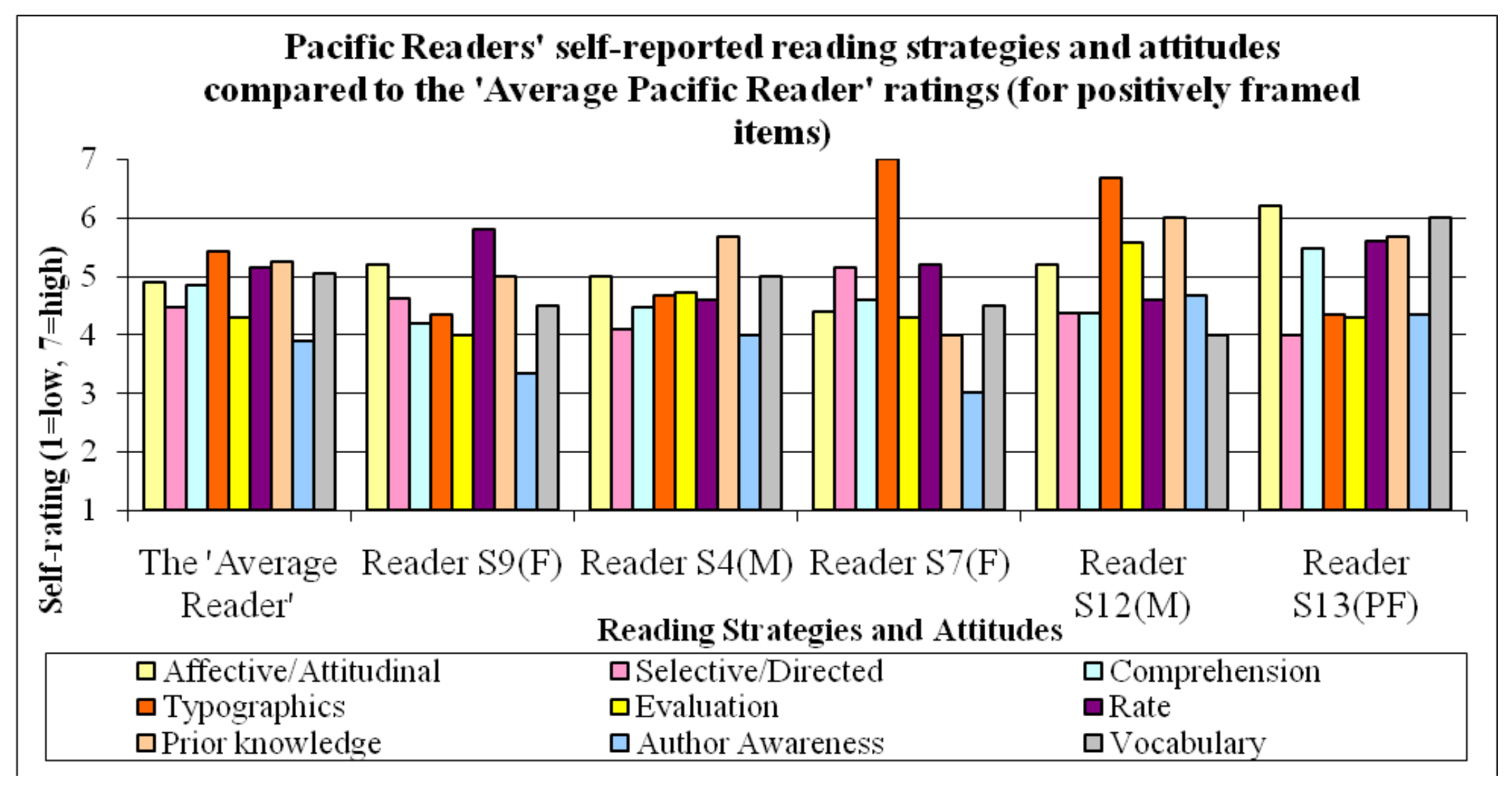

Figure 7.6: Differences between Course A readers in self reported use of positively framed inventory items 


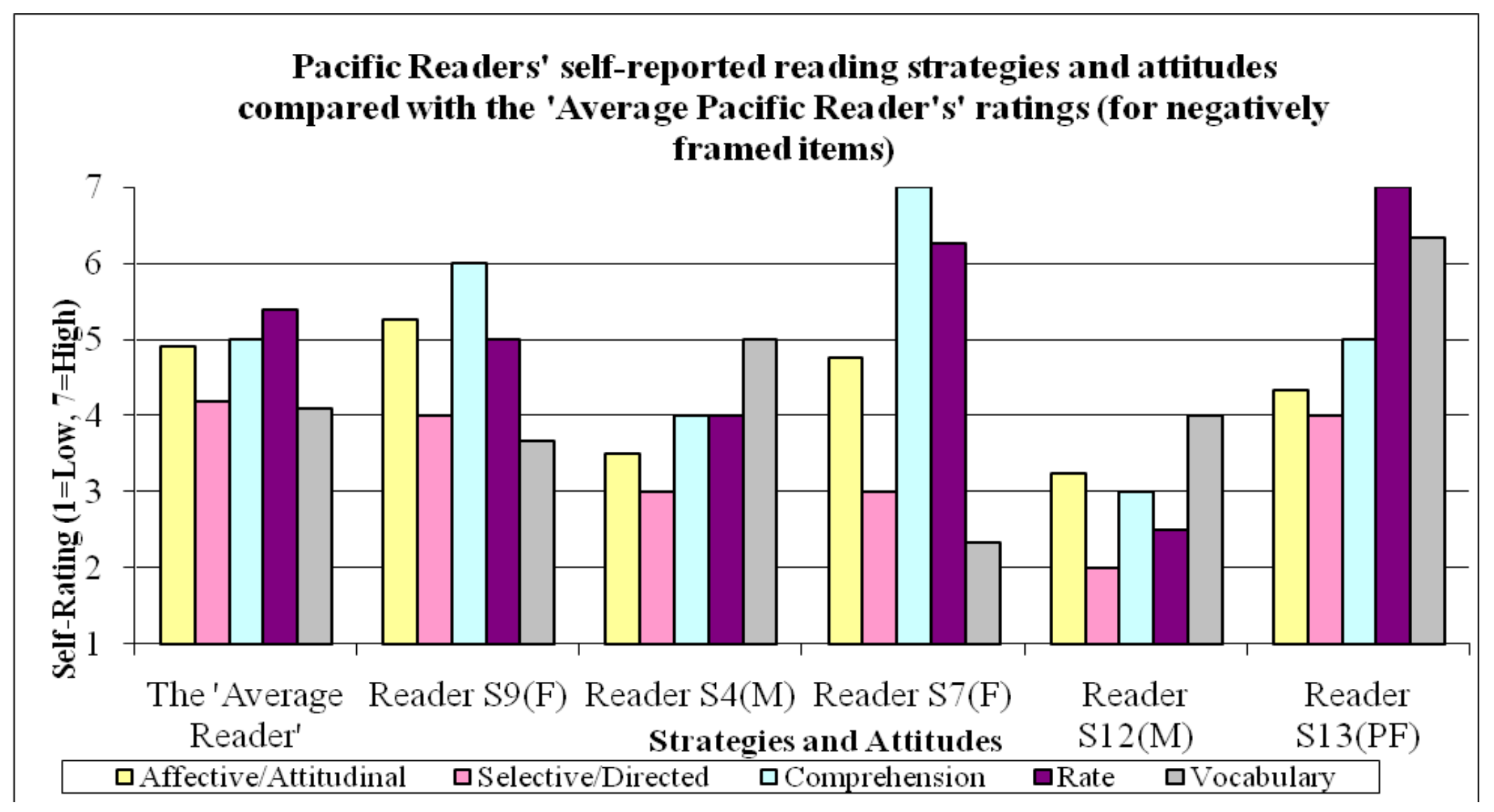

Figure 7.7: Differences between Course A readers in self reported use of negatively framed inventory items 
The inventory was taken by 12 first year Pacific Readers. The aggregate results of the Inventory have allowed the generation of a reading profile of a typical Pacific reader based on the averages of the Pacific participant group in this study. It is against this group average that each of the case study Course A students were able to be compared to reveal overall patterning of the group, and interesting individual variations and idiosyncracies. The results thus allow identification of patterns in reported usage of the various strategy types across this group of readers, and insights into each Reader's self-reported reading persona.

\section{(7.4.2) The Pacific participants'group averages and typical patterning revealed}

The average strategies profile of the Pacific participant group provides a tentative understanding of general trends in the characteristics of these first year Course A Readers of Pacific descent. This profile is likely to vary from institution to institution, year to year, age group to age group, and amongst educational backgrounds, however the snapshot provided in this study does yield valuable insights into this group and is indicative of Pacific students nationally.

In terms of the strategies and attitudes considered to be characteristic of successful readers the Pacific participant group averages demonstrate all strategy types except author awareness at a frequency above the mid-point (a rating of 4) on the 7-point rating scale. These ratings above 4 would be interpreted as potential strengths in this group's overall profile. The order of reported use of positive strategy and attitude characteristics from most used to least used, based on overall combined Reader averages, is shown in Figure 7.8 below. 


\begin{tabular}{|l|l|}
\hline & $\begin{array}{l}\text { The Pacific participants' } \\
\text { group average ratings } \\
\text { for Inventory category } \\
\text { (1=Never used, 7=Always } \\
\text { used) }\end{array}$ \\
\hline Typographics & 5.42 \\
\hline Prior knowledge & 5.24 \\
\hline Rate & 5.14 \\
\hline Vocabulary & 5.04 \\
\hline Affective/Attitudinal & 4.89 \\
\hline Comprehension & 4.83 \\
\hline Selective/Directed & 4.45 \\
\hline Evaluation & 4.29 \\
\hline Author Awareness & 3.87 \\
\hline
\end{tabular}

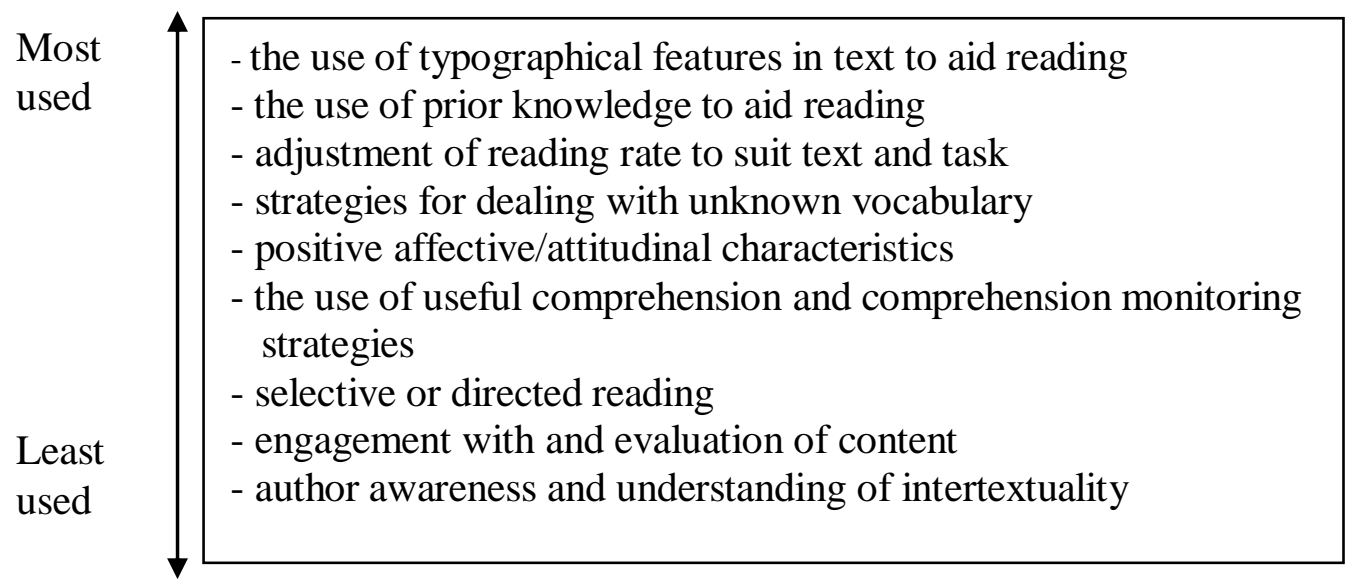

Figure 7.8: 'Most used' to 'least used' reading strategies according to readers' combined average self-ratings for positively framed items

The findings of this study indicate that the utilization of typographical features while reading was the strategy that was, on average, the most utilized by these Pacific Readers. This concurs with Stanovich's (1980, p.50) observation that "good readers paid more attention to graphic information" and were more sensitive to typography, and also suggests that many of these Pacific students may be visually oriented learners. This commonly shared strength can be 
utilized and harnessed for strategic and successful reading by these Pacific Readers.

On the other hand, the group was also found to possess limitations in their awareness of authors and intertextuality which are likely to need specific development to enable and enhance success in higher education where an awareness and utilization of these is particularly useful. The issue of author awareness is of particular research interest in the context of higher education. In earlier levels (junior high school) Krieger (1990, p.618) asserts that her students "weren't even aware of the writer", and observes that there did not appear to be any interaction between the readers and the author, and that many were missing "a sense of who wrote the book, how, and why." These results indicate that this trend does carry into higher education for these Readers. Furthermore, the central importance of intertextuality to higher levels of reading is confirmed in studies by Moore and Morton (2005) and Turner (2005) in Australia and New Zealand which "respectively confirm that assessment at universities across all disciplines involves synthesizing knowledge derived from multiple textual sources" (cited in Wilson, 2009, p.4).

In terms of the characteristics found to be typical of less successful readers, the Pacific participant group's averages reveal that they reported attitudinal and affect-related issues at a frequency greater than the midpoint on the selfreporting scale. Their self-reports also demonstrate that they quite frequently utilize less successful comprehension and reading rate related strategies and these are areas potentially in need of development. The order of reported use of less successful strategy and attitude characteristics from most used (and therefore most potentially problematic) to least used is shown in Figure 7.9 below. 


\begin{tabular}{|l|l|}
\hline & $\begin{array}{l}\text { The Pacific participants } \\
\text { group's average ratings for } \\
\text { Inventory category } \\
(1=\text { Never used, 7=Always } \\
\text { used })\end{array}$ \\
\hline Rate & 5.39 \\
\hline Comprehension & 5 \\
\hline Affective/Attitudinal & 4.9 \\
\hline Selective/Directed & 4.18 \\
\hline Vocabulary & 4.09 \\
\hline
\end{tabular}

\begin{tabular}{|c|c|}
\hline Most & inappropriate reading rate adjustment and monitoring strategies \\
\hline used & $\begin{array}{l}\text { - inappropriate comprehension and comprehension monitoring } \\
\text { strategies }\end{array}$ \\
\hline & $\begin{array}{l}\text { - possessing affective and attitudinal characteristics that may inhibit } \\
\text { successful reading }\end{array}$ \\
\hline & - reading approaches that are not particularly selective or directed \\
\hline $\begin{array}{l}\text { Least } \\
\text { used }\end{array}$ & $\begin{array}{l}\text { - vocabulary strategies that may not be helpful for reading (i.e. overly } \\
\text { time-consuming) }\end{array}$ \\
\hline
\end{tabular}

Figure 7.9: 'Most used' to 'least used' reading strategies according to readers' combined average self-ratings for negatively framed items

The results suggest that the strategies and attitudes potentially the most problematic for the Pacific participant group on the whole include demonstrating inappropriate reading rate adjustment and monitoring strategies, inappropriate comprehension and comprehension monitoring strategies, and possessing affective and attitudinal characteristics that may inhibit successful reading.

If this group can be taken to be indicative of typical first year Pacific readers, then these results suggest that it would be beneficial for Pacific student readers entering higher education to undertake development activities to: 
- strengthen the useful strategies and attitudes that they already possess and their ability to monitor their usefulness in any given situation and make the necessary adjustments

- raise author awareness and concepts of intertextuality in the students in ways appropriate to their discipline

- raise the student's awareness of the types of comprehension strategies they typically utilize, and identify those that may be hindering their reading within their new academic reading environment. They would also require greater awareness of the need to acquire strategies better suited to the texts and tasks encountered. In a local NZ context, this may involve the development of a greater awareness of those "specific, learned procedures that foster active, competent, self-regulated and intentional reading” (Trabasso \& Bouchard, 2002, p.177).

- raise the student's awareness of the types of reading rate-related strategies that may hinder their academic reading within their new academic reading environment and raise awareness of the need to acquire strategies better suited to the texts and tasks encountered in it.

- examine, recognize and begin to address current reading attitudes and affective reactions to text that may be hindering their academic reading, and begin to build positive associations with academic reading.

In addition to this general picture, each Pacific student surveyed was found to possess unique combinations of traits that make them who they are as Readers. Likewise, each Reader also has their own unique development plan customized to their profile's results. The scope of the thesis has not allowed 
the complete profiles of all six Course A Readers to be displayed, however due to the restrictions of the thesis size, two Readers' holistic profiles are presented (S7(F) and S13(F)) in Chapter 9. These two readers have been selected because while both would be classified as 'Samoan females', they do in fact demonstrate clearly distinct reading personae, and would thus require personal development which would differ in some quite marked ways. They therefore make interesting comparative cases through which to demonstrate the usefulness of the profiling system put forward in this thesis, and to illustrate the uniqueness of the reader.

However in all this discussion of 'successful' and 'less successful' strategy use it should be borne in mind that strategies are seldom inherently 'bad' or 'good' but that their use makes them so. While it has been documented that the presence of some types of reading strategies are more commonly associated with 'good readers', ultimately however it perhaps should not be said that one strategy, or type of strategies is 'best' for academic reading. Rather, the quality of a successful academic reader is that they possess a wide array of strategies and are able to flexibly select, use and monitor those that are appropriate to the task at hand (Simpson \& Nist, 2000). This understanding is supported by researcher practitioners in the actual content fields at university. White (2004, p.39) for example stresses that students should be assisted to learn to adjust their reading speeds and styles according to clearly defined reading objectives and to the nature of the text itself. These different characteristics of text and task could require any one of a very wide range of reading responses, and oftentimes a combination of responses are necessary even within the one reading experience. 


\section{Chapter Summary}

In this chapter the findings gathered from the main strands of Pacific student Reader profiling undertaken in this study have been presented. This study has revealed that, in their first year of study at the case study New Zealand University, the Pacific Readers:

- came from widely varying reading and literacy backgrounds. They differed often in terms of the roles of English and other Pacific languages within the home, their attitudes to and habits of reading throughout childhood, and the literacy-related features of their home environments. A number of similarities also emerged across Readers and these themes have also been presented.

- varied widely in their vocabulary knowledge, and on average, had less estimated vocabulary knowledge in the Vocabulary Size Test than a sample of their non-Pacific peers on all but four of the fourteen levels. Knowledge of the UWL items was limited for some Readers and from these results it was reasoned that few would reach the thresholds indicated in the research literature for successful independent comprehension of text.

- demonstrated an average Reading Rate for this Task which was found to be comparable with other studies in higher education. Much variation was found amongst the Readers, and Reading Rate was tentatively found to be related to Recall success and to the existence of certain characteristics within both the Text and the Reader themselves.

- $\quad$ possessed in their patterns of reading strategy use, definite preferences for certain types of academic reading strategies while seldom utilizing the potential benefits of others. They also possessed some characteristics that 253 
indicate they may not be utilizing certain strategies in the most appropriate ways for higher education.

What we now know about Pacific first year student Readers' backgrounds, Vocabulary sizes, patterns of Reading Strategy use and Reading Rate allows a more accurate understanding of their academic reading strengths and how best to work on their areas for development.

The chapters thus far have presented the results of investigation of the three main elements of the central research framework - the Texts, the Tasks and now the Readers. The next chapter then moves to focus on some of the facets of the interaction between two of these - the Reader and the Text - by presenting findings on these Pacific Readers' affective and perceptive responses to text as well as indications of how, and how well, they undertook the task of reading to understand and remember. 


\section{CHAPTER 8}

\section{THE READER $\leftrightarrow$ TEXT INTERACTION: FIRST YEAR PACIFIC STUDENTS' AFFECTIVE REACTIONS TO TEXTS \& PERFORMANCE IN FREE RECALL OF ACADEMIC TEXT}

In this chapter the results of investigation into the interaction between Text and Reader are represented. Examination of this interaction was made possible by holding the Task factor constant. The typical reading Task that has been chosen for the purpose of closely examining the interaction of Pacific Readers and academic Text is that of 'reading to understand and remember'. This basic academic reading Task was identified as central to the first year academic reading in the Courses examined in Chapter 6.

This chapter addresses the following: Research Question 3(a(v)): What are the characteristics of first year Pacific students in terms of their Affective and Perceptive responses to text? Research Question 3(b): What is the nature of the Pacific students' interactions with academic text whilst performing a typical reading task with authentic texts from their Course?

The chapter is presented as follows:

Pacific Readers' perceptions of Passage Interest and Difficulty General perceptions of Interest across Passages A6-All General perceptions of Difficulty across Passages A6-A11 Overall Affective/Perceptive Responses to Course A Texts Readers' Free Recall performance Overall Amounts of IU Recall Pacific Readers' Perceptions of Interest \& Difficulty \& \% IU Recall

How Course A Pacific Readers characteristically interacted with the academic texts during the Free Recall tasks 
(8.3.1) Patterns of Free Recall of Text: Map of Idea Units (IU) recalled

(8.3.2) Mapping of Recall Frequency for Idea Units

(8.3.3) Patterns of IU omission in Free Recalls

(8.4) Post-task Interview Findings: What the Readers themselves reported about how they read.

\section{(8.1) Pacific Readers' Perceptions of Passage Interest and Difficulty}

Earlier thematic analysis of Reader interviews revealed that these Pacific students' affective responses to texts were an important feature of their academic reading experience. The two affective and perceptive responses chosen for closer investigation in this study were the Readers' perceptions of (1) Passage Interest and (2) Passage Difficulty.

\section{(8.1.1) General Perceptions of Interest for Passages A6-A11}

The Pacific Readers' overall perceptions of Interest for Passages A6-A11 were rated on a scale as follows:

1. low Interest

2. low/mid Interest

3. $\mathrm{mid} /$ low Interest

4. mid Interest

5. $\mathrm{mid} /$ high Interest

6. high/mid Interest

7. high Interest

Individual Reader differences in affective response to texts emerged quite clearly. The table below indicates that in many cases the same Free Recall Passage provoked quite varied Interest responses amongst the case study Readers. These are shown in Table 8.1 below. 
Table 8.1: Course A Pacific readers' interest ratings for free recall passages

\begin{tabular}{|l|l|l|l|l|l|l|l|}
\hline & $\begin{array}{l}\text { S9(F) } \\
\text { Perceived } \\
\text { Interest }\end{array}$ & $\begin{array}{l}\text { S4(M) } \\
\text { Perceived } \\
\text { Interest }\end{array}$ & $\begin{array}{l}\text { S5(M) } \\
\text { Perceived } \\
\text { Interest }\end{array}$ & $\begin{array}{l}\text { S7(F) } \\
\text { Perceived } \\
\text { Interest }\end{array}$ & $\begin{array}{l}\text { S12(M) } \\
\text { Perceived } \\
\text { Interest }\end{array}$ & $\begin{array}{l}\text { S13(PF) } \\
\text { Perceived } \\
\text { Interest }\end{array}$ & $\begin{array}{l}\text { Average } \\
\text { Interest } \\
\text { Rating } \\
\text { for } \\
\text { Passage }\end{array}$ \\
\hline A6 & 5 & 5 & 5 & 4 & 1 & 5 & 4.2 \\
\hline A7 & 7 & 1 & - & 1 & 1 & 7 & 3.4 \\
\hline A8 & 1 & 6 & - & 4 & 5 & 4 & 4.0 \\
\hline A9 & 5 & 7 & - & 4 & 1 & 2 & 3.8 \\
\hline A10 & 1 & 1 & - & 1 & 1 & 5 & 1.8 \\
\hline A11 & 1 & 2 & - & 4 & 2 & 4 & 2.6 \\
\hline $\begin{array}{l}\text { Reader } \\
\text { Average }\end{array}$ & 3.3 & 3.6 & 5 & 3 & 1.8 & 4.5 & \\
\hline
\end{tabular}

The variations in the perceived Interest of the various Passages must be due to Reader factors, since the Passage itself does not change in any way but Readers' Interest ratings obviously do. It is likely that it is the semantic character of these texts that are the primary Interest-related feature of texts.

\section{(8.1.1) General Perceptions of Difficulty across Course A Passages A6-A11}

Similarly to Interest, perceptions of Difficulty for Passages A6-A11 were rated on a scale as follows:

1. low Difficulty

2. low/mid Difficulty

3. mid/low Difficulty

4. mid Difficulty

5. mid/high Difficulty

6. high/mid Difficulty

7. high Difficulty 
When student Readers' Difficulty scores for all the Passages are averaged, an indication of the degree of overall challenge these authentic texts presented can be seen in Table 8.2 below.

Table 8.2: Course A Pacific readers' difficulty ratings for free recall passages

\begin{tabular}{|l|l|l|l|l|c|c|l|}
\hline & $\begin{array}{l}\text { S9(F) } \\
\text { Perceived } \\
\text { Difficulty }\end{array}$ & $\begin{array}{l}\text { S4(M) } \\
\text { Perceived } \\
\text { Difficulty }\end{array}$ & $\begin{array}{l}\text { S5(M) } \\
\text { Perceived } \\
\text { Difficulty }\end{array}$ & $\begin{array}{l}\text { S7(F) } \\
\text { Perceived } \\
\text { Difficulty }\end{array}$ & $\begin{array}{l}\text { S12(M) } \\
\text { Perceived } \\
\text { Difficulty }\end{array}$ & $\begin{array}{l}\text { S13(PF) } \\
\text { Perceived } \\
\text { Difficulty }\end{array}$ & $\begin{array}{l}\text { Average } \\
\text { Difficulty } \\
\text { Rating for } \\
\text { Passage }\end{array}$ \\
\hline A6 & 1 & 1 & 2 & 2 & 1 & 5 & 2 \\
\hline A7 & 1 & 1 & - & 5 & 1 & 1 & 1.8 \\
\hline A8 & 7 & 1 & - & 4 & 1 & 5 & 3.6 \\
\hline A9 & 1 & 1 & - & 1 & 1 & 1 & 1 \\
\hline A10 & 1 & 4 & - & 1 & 1 & 1 & 1.6 \\
\hline A11 & 2 & 1 & - & 1 & 1 & 1 & 1.2 \\
\hline $\begin{array}{l}\text { Reader } \\
\text { Average }\end{array}$ & 2.2 & 1.5 & 2 & 2.3 & 1 & 2.3 & \\
\hline
\end{tabular}

Of note in these results are the scores of S7(F) and S13(PF) who found the Course A Passages more difficult on average than other Course A Pacific Readers. It is noteworthy that $\mathrm{S} 13(\mathrm{PF})$ also rated the Passages as being of higher overall Interest than all other Readers, whereas S7(F), who here shows some of the highest ratings for Difficulty, was amongst the lowest in terms of average Interest ratings for these Passages. This is evidence yet again of how two Readers may exhibit similar characteristics on a certain profile measure but do in fact differ greatly in other features of their reading personae. This highlights the necessity that Reader profiling is holistic, comprehensive, and multifaceted in its approach. 


\section{(8.1.2) Overall Affective/Perceptive Responses to Course A Texts}

It is apparent that for the reading assigned in Course A, the overall affective and perceptive reading responses of the Pacific case study Readers were quite varied. Table 8.3 below records each Reader's characteristic affective and perceptive responses.

Table 8.3: Course A Pacific readers' perceptive and affective responses to texts

\begin{tabular}{|l|l|}
\hline Reader & $\begin{array}{l}\text { Perceptive and Affective Responses. } \\
\text { Course A Texts were found to be: }\end{array}$ \\
\hline S7(F) & of low Interest and high Difficulty \\
\hline S13(PF) & of high Interest and high Difficulty \\
\hline S9(F) & of medium level Interest and medium level Difficulty \\
\hline S5(M) & Insufficient data was collected to gauge overall reactions to Course A texts \\
\hline S4(M) & of reasonably high Interest and low Difficulty \\
\hline S12(M) & of low Interest and also low Difficulty \\
\hline
\end{tabular}

These varying combinations of Interest and Difficulty suggest that these Readers probably experienced uniquely varying levels of enjoyment, motivation or frustration in academic reading in this Course. Comparison of these affective response indicators with what the Readers' end-of-Course interviews revealed about their academic reading experiences reveals that these combinations are fairly accurate predictors of their academic reading experiences throughout the Course. Moreover, for some Readers they also provide an accurate indicator of their ultimate success in the Course (see Chapter 10). 


\section{(8.2) Reader's Free Recall Performance}

\section{(8.2.1) Overall Amounts of IU Recall}

Table 8.4 below shows the six Course A Readers' overall performance on the Free Recall task in terms of the percentage of Idea Units (IU) recalled from the sample Passages. Unfortunately due to unforeseen technical problems, and to the unavailability of some participants during the data collection period (much of which was during term time), certain portions of the data are missing.

Table 8.4: Course A Pacific readers' overall amounts of IU recall

\begin{tabular}{|l|l|l|l|l|l|l|}
\hline & $\begin{array}{l}\text { Reader } \\
\text { S9(F) }\end{array}$ & $\begin{array}{l}\text { Reader } \\
\text { S4(M) }\end{array}$ & $\begin{array}{l}\text { Reader } \\
\text { S5(M) }\end{array}$ & $\begin{array}{l}\text { Reader } \\
\text { S7(F) }\end{array}$ & $\begin{array}{l}\text { Reader } \\
\text { S12(M) }\end{array}$ & $\begin{array}{l}\text { Reader } \\
\text { S13(PF) }\end{array}$ \\
\hline Passage A6\% Recall & - & $41 \%$ & $38 \%$ & $24 \%$ & $26 \%$ & $7 \%$ \\
\hline Passage A7\% Recall & $36 \%$ & - & - & $29 \%$ & $32 \%$ & $29 \%$ \\
\hline Passage A8\% Recall & $14 \%$ & - & - & $19 \%$ & $30 \%$ & $19 \%$ \\
\hline Passage A9\% Recall & $26 \%$ & - & - & - & $26 \%$ & $17 \%$ \\
\hline Passage A10\% Recall & $27 \%$ & - & - & $35 \%$ & $24 \%$ & $15 \%$ \\
\hline Passage A11\% Recall & $32 \%$ & - & - & $29 \%$ & $26 \%$ & $6 \%$ \\
\hline
\end{tabular}

To illustrate the variations amongst the Readers in task success, the overall range of \% IU Recall across both the Free and Prompted Recall protocols of the Readers in Course A is represented by the shaded areas in Table 8.5. (Note that Readers S4(M) and S5(M) have not been included as they each undertook only one recall task due to other commitments during the time of data collection for Free Recalls.) 
Table 8.5: Course A Pacific readers' ranges of percentage IU recall from Free Recall of Course A passages

\begin{tabular}{|l|l|l|l|l|l|l|l|l|l|l|}
\hline & $\begin{array}{l}0-10 \\
\%\end{array}$ & $\begin{array}{l}10-20 \\
\%\end{array}$ & $\begin{array}{l}20-30 \\
\%\end{array}$ & $\begin{array}{l}30-40 \\
\%\end{array}$ & $\begin{array}{l}40-50 \\
\%\end{array}$ & $\begin{array}{l}50-60 \\
\%\end{array}$ & $\begin{array}{l}60-70 \\
\%\end{array}$ & $\begin{array}{l}70-80 \\
\%\end{array}$ & $\begin{array}{l}80-90 \\
\%\end{array}$ & $\begin{array}{l}90- \\
100 \\
\%\end{array}$ \\
\hline $\begin{array}{l}\text { S9(F) } \\
10-45 \%\end{array}$ & & & & & & & & & & \\
\hline $\begin{array}{l}\text { S7(F) } \\
15-65 \%\end{array}$ & & & & & & & & & & \\
\hline $\begin{array}{l}\text { S12(M) } \\
20-80 \%\end{array}$ & & & & & & & & & & \\
\hline $\begin{array}{l}\text { S13(PF) } \\
5-50 \%\end{array}$ & & & & & & & & & & \\
\hline
\end{tabular}

When comparisons between the average recall percentages achieved in the Free and Prompted Recalls were carried out, the Pacific Readers were clearly able to recall more IU in the Prompted Recall (average 49.8\%) than they were able to in the Free Recall (average 29.3\%). A possible explanation for this may be Chang's (2006, p. 520) findings that, when Free Recall was compared with a translation task, "the requirement of memory in the recall task hinders test-takers' ability to demonstrate fully their comprehension of the reading passage". Thus, the Free Recall protocol is limited in that it does not fully indicate the extent of the Reader's comprehension. Nevertheless, it is still a "powerful tool for tracking psychological processes" (Hayes, 1989, p. 69, cited in Chang, 2006, p.521) and has proven valuable in this study for shedding light on these Pacific Readers' meaning-making approaches to text.

\section{(8.2.2) Pacific Readers' Perceptions of Interest \& Difficulty \& \% IU Recall}

In observing the relationship between Readers' \% IU Recall and their perceptions of Passage Interest and Difficulty when undertaking the task of 'reading to understand and remember' the following results (in Figure 8.1) were obtained. 


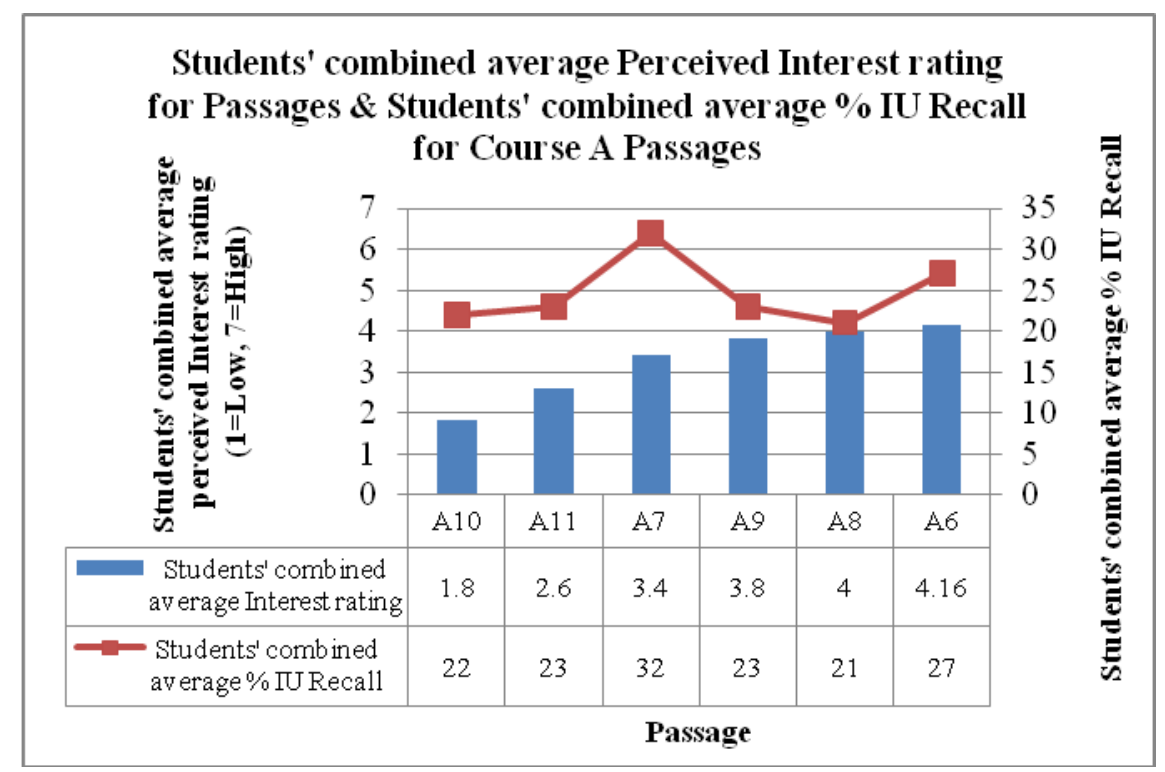

Figure 8.1: Course A Pacific readers' combined average perceived interest ratings \& percentage of IU recall for Course A passages

Somewhat surprisingly, the results do not reveal any overall systematic relationship between Readers' perceptions of Interest and their performance on the IU Recall task. Given the importance of interest hitherto observed amongst Readers, it might be expected that a clear relationship would emerge; its absence is most likely due to the considerable individual variation found amongst the Readers.

For $\mathrm{S} 9(\mathrm{~F}), \mathrm{S} 12(\mathrm{M})$ and $\mathrm{S} 13(\mathrm{PF})$, increased perceptions of text Interest corresponded to greater success in the Recall task. On the other hand it was notable that for S4(M), S7(M) and S5(M) increased Interest was related to poorer performance in IU Recall. This suggests that, once aroused, Interest is an affective reaction to text that may have powerful effects on task performance, but that these may be either positive or negative. For some, 
Interest may be vital to task success (such as S12(M)), for some it may be an added bonus (such as S9(F)), while for some it may even serve as a distracter from what is perceived to be the largely mechanical task at hand (such as S7(F)). However with so few cases only very tentative interpretations can be offered at this point without further investigation.

The results of comparison of Difficulty perceptions and Recall are outlined in Figure 8.2. below

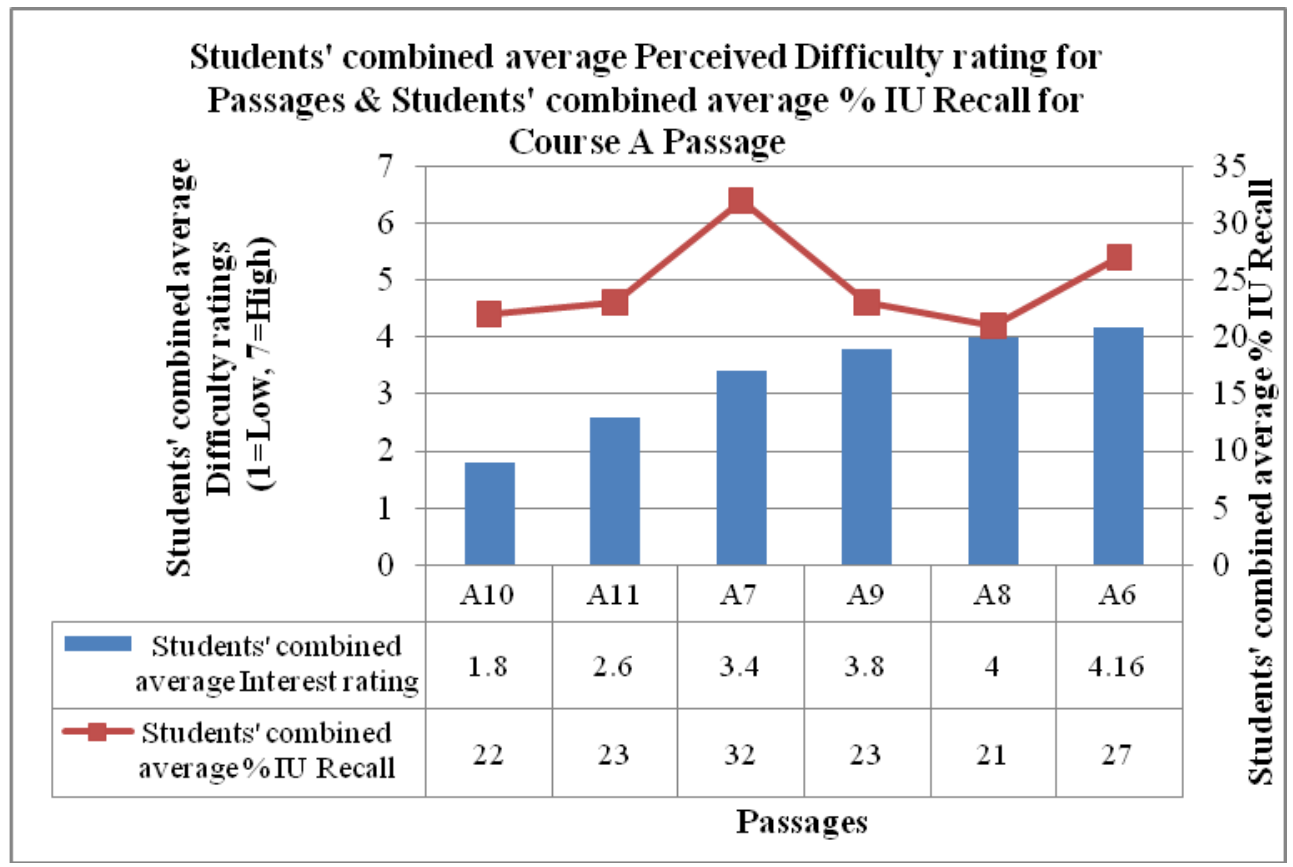

Figure 8.2: Course A Pacific readers' combined average perceived difficulty ratings \& percentage IU recall for Course A passages

The relationship between Readers' perceptions of text Difficulty and their performance on the IU Recall was also varied. On an individual basis, for half the Course A Readers (S9(F), S4(M), S5(M)) texts that were perceived as being of greater Difficulty were the ones on which they scored lower Recall. 
For Readers S7(F) and S13(PF), on the other hand, reactions of increased perceived text Difficulty appeared to relate to greater Recall success. For S12(M), his Difficulty perceptions did not appear to correlate in any way with any other features measured.

The results suggest that these Pacific Readers' perceptions of the Difficulty of a text had varying effects on them in their task performance. For some Readers it appeared that increased text Difficulty spurred them on to greater success in Recall (possibly as a result of greater application of conscious effort to the task in response to the perceived challenge it presented), while for others it led to decreased task efficacy (perhaps as negative affective responses affected performance). Other findings within the reading profiles of these Readers may also provide further possible reasons for this observation. Knowing the characteristic effect of these affective and perceptive reactions to text on a particular Reader is a valuable key to understanding what facilitates or hinders individual academic reading Task performance.

The findings thus far appear to support the idea that the manner in which a Reader responds affectively to a text is important, and that there is considerable individual variation amongst Readers in what impact this has on their ultimate task success. This reveals (1) the uniqueness of the Reader, and (2) the importance of understanding them and seeing their responses to texts as being shaped by many features within their reading persona.

Empirical exploration of the role of interest in university level reading and learning has generally indicated that interest may be key to determining how students respond to text and how they process information. It has been suggested that parts of text found to be interesting are processed in a different way from what is not found to be interesting. Specifically, Lee (2009, p.172) 
found that interest had "subtle but systematic effects" on reading comprehension and that "reading passages that do not capture the interest of an L2 reader would risk the reader's retention of smaller details". Shearer, Lundeberg and Coballes-Vega (1997, p.592) also observed that "capable adult readers are especially adept at reading in areas matched to their knowledge base and interest, often changing their cognitive and affective processing in response to the text they are reading”. Likewise, Garner, Alexander, Gillingham, Kulikowich and Brown (1991) also found that when readers had little background knowledge on a topic then their levels of interest in it had a considerable effect on their ability to recall information from the text.

In early studies of reading and interest Belloni and Jongsma (1978) found that compulsory school aged students comprehended texts that they found interesting better than texts that they did not find interesting, and that they were able to transcend frustration levels if the text was interesting. This belief in the ability of positive affective response to text (such as Interest) serving to counterbalance or to moderate the impact of the negative affective response of perceived Difficulty is also observed in some of these Pacific Readers. These results offer tentative support for the idea that Interest may mitigate Difficulty and facilitate comprehension and recall for some of these Readers. This would presuppose that these affective responses of interest and difficulty represent quite separate responses and can coexist together as they appear to have done in these Pacific Readers' responses to Passages such as A6 and A8 - both of which demonstrated high average ratings for both Interest and Difficulty simultaneously.

In terms of Difficulty and Reader response, these findings are consistent with the literature that attests to the links between perceptions of difficulty and 
negative affective response to text, which in turn have a range of negative flow-on effects on readers and their reading habits and ultimately on the levels of success of their reading (Guthrie, Coddington \& Wigfield, 2009).

Interestingly however, despite the clear importance of these affective responses, they did not relate directly to task success in any systematic way. Instead, their importance is likely to have been linked to their contribution to wider affective, attitudinal and motivation effects experienced by the Pacific Readers.

\section{(8.3) How Course A Pacific Readers Characteristically Interacted with the Academic Texts during the Free Recall Tasks}

This section presents the findings of a more in-depth analysis of the Readers' Free Recall performance. The overall percentage of IU recalled from Passages gives a general understanding of the Readers' relative task success, but further analysis allows insight into the amount, patterning and nature of their recall. By closely analyzing features of both the process and the product of the reading task, this phase of the study utilized IU mapping and transcript analysis to generate insights into the interaction between the Pacific Reader and the authentic Texts they encounter in their study, and how (and how well) they carried out the set task.

\section{(8.3.1) Patterns of Free Recall of Text: Map of Idea Units (IU) Recalled}

Patterns of IU Recall for Course A Pacific students during Free Recall of authentic Course A text samples were recorded, and for the sake of space constraints, Passage A6 is presented Table 8.6 below and the IU recall patterns of A7-A11 are presented in Appendix 7. Due to unforeseen technical issues with the recording device used, only 4 of the 6 Course A participants' Free 
Recalls were recorded. Thus there are only four columns on the right of the tables representing these four Readers, and in Passage A6 the four differ from the four represented by Passages A7-A11. However, despite these limitations, these tables do allow valuable insights into the characteristics of both the Texts and the Readers. 
Table 8.6: Existence of IU in Course A Pacific readers' free recall transcripts for

Passage A6 passage A6

\begin{tabular}{|c|c|c|c|c|c|}
\hline \multirow[t]{2}{*}{$\begin{array}{l}\text { IU Label } \\
\#\end{array}$} & \multirow{2}{*}{$\begin{array}{l}\text { Sentence IU is } \\
\text { found in } \\
\#\end{array}$} & \multicolumn{4}{|c|}{ Existence of IU in Student Free Recall } \\
\hline & & $\mathrm{S} 4$ & S5 & $\mathrm{S} 12$ & $\mathrm{~S} 13$ \\
\hline 1 & s1 & & & & $\checkmark$ \\
\hline 2 & s2 & & & $\checkmark$ & \\
\hline 3 & s3 & & $\checkmark$ & $\checkmark$ & \\
\hline 4 & s3 & $\checkmark$ & & & \\
\hline 5 & s4 & $\checkmark$ & $\checkmark$ & $\checkmark$ & \\
\hline 6 & s5, s16 & $\checkmark$ & $\checkmark$ & $\checkmark$ & \\
\hline 7 & s5, s16 & & & & \\
\hline 8 & s5, s16 & $\checkmark$ & & & \\
\hline 9 & s5, s16 & $\checkmark$ & & $\checkmark$ & \\
\hline 10 & s5, s16 & $\checkmark$ & $\checkmark$ & & \\
\hline 11 & s6, s17 & $\checkmark$ & $\checkmark$ & $\checkmark$ & $\checkmark$ \\
\hline 12 & s6, s17 & & & & \\
\hline 13 & s6, s17 & $\checkmark$ & $\checkmark$ & & \\
\hline 14 & s6, s17 & & $\checkmark$ & & \\
\hline 15 & s6, s17 & $\checkmark$ & & & \\
\hline 16 & s6, s17 & & $\checkmark$ & & \\
\hline 17 & s7 & & & & \\
\hline 18 & s8 & $\checkmark$ & $\checkmark$ & & \\
\hline 19 & s8 & & & & \\
\hline 20 & s8 & & & & \\
\hline 21 & s9 & & & & \\
\hline 22 & s10 & & & & \\
\hline 23 & $\mathrm{~s} 10$ & & & & \\
\hline 24 & s10 & & & & \\
\hline 25 & s11 & $\checkmark$ & $\checkmark$ & $\checkmark$ & \\
\hline 26 & s12 & $\checkmark$ & $\checkmark$ & & $\checkmark$ \\
\hline 27 & s12 & $\checkmark$ & $\checkmark$ & & \\
\hline 28 & s12 & & & & \\
\hline 29 & s12 & & & & \\
\hline 30 & s13 & $\checkmark$ & $\checkmark$ & $\checkmark$ & \\
\hline 31 & s13 & & & & \\
\hline 32 & s13 & & & & \\
\hline 33 & s14 & & $\checkmark$ & & \\
\hline 34 & s14 & & $\checkmark$ & & \\
\hline 35 & s14 & $\checkmark$ & & & \\
\hline 36 & s15 & & & & \\
\hline 37 & s15 & & & & \\
\hline 38 & s18 & & & $\checkmark$ & \\
\hline 39 & s18 & & & $\checkmark$ & \\
\hline 40 & s19 & & & & \\
\hline 41 & s19 & $\checkmark$ & & & \\
\hline
\end{tabular}


Analysis of these tables reveals that:

- There are sections of each text that most Readers have been able to recognize as central to the text's meaning, and clear clustering of IU Recall is observable around these sections of text.

- In addition to the general similarities, there were also differences between the individual Readers in the IU that they recalled. Some Readers occasionally selected IU that other Readers did not, while others consistently recalled IU that were different from other Readers.

- It is also clear that there are some sections of all the Passages from which no students recalled any IU. This is investigated further in a following section of this chapter.

In addition to an overall understanding of how students respond to the text and select and commit to memory certain parts of it, analysis of the Free Recall patterns provides insights into Reader idiosyncrasies in patterns of text Recall.

\section{(8.3.2) Mapping of Recall Frequency for Idea Units}

To understand the Reader and Text interaction, it is helpful to observe patterns at the sentence level of the IU which were and were not recalled by students. Table 8.7 below visually demonstrates which elements of the sample Passages in Course A were recalled by the Pacific reader participants, and how often, with the darker shading representing more recall and no shading representing no recall by participants. For the sake of brevity only Passage A6 is shown here, and Passages A7-A11 can be found in Appendix 8. 


\section{Passage A6}

Table 8.7: Map of frequency of IU recall patterns within sentences in Course A, passage A6

KEY

\begin{tabular}{|c|c|c|}
\hline & \multirow{2}{*}{ Exists in ALL 4 student recalls } & \\
\hline & & ts in ALL 4 student recalls \\
\hline & & Exists in 2 out of 4 student recalls \\
\hline & & Exists in 1 out of 4 student recalls \\
\hline & & Does NOT exist in ANY student recalls \\
\hline & $\begin{array}{l}\text { Sentence } \\
\text { length } \\
\text { (no. of } \\
\text { words) }\end{array}$ & \\
\hline s1 & 19 & $\begin{array}{l}\text { Communication and related interpersonal skills must be at the forefront of } \\
\text { any attempt to develop managerial and leadership expertise. }\end{array}$ \\
\hline s2 & 13 & $\begin{array}{l}\text { Among these essential skills, the ability to deal with interpersonal conflicts is } \\
\text { critical. }\end{array}$ \\
\hline s3 & 13 & $\begin{array}{l}\text { Formally defined, conflict is a disagreement between people on substantive } \\
\text { or emotional issues. }\end{array}$ \\
\hline s4 & 14 & $\begin{array}{l}\text { Managers and leaders spend a lot of time dealing with conflicts of various } \\
\text { forms. }\end{array}$ \\
\hline s5 & 23 & $\begin{array}{l}\text { Substantive conflicts involve disagreements over such things as goals; the } \\
\text { allocation of resources; the distribution of rewards, policies and procedures; } \\
\text { and job assignments. }\end{array}$ \\
\hline s6 & 18 & $\begin{array}{l}\text { Emotional conflicts result from feelings of anger, distrust, dislike, fear and } \\
\text { resentment, as well as from personality clashes. }\end{array}$ \\
\hline s7 & 10 & Both forms of conflict can cause problems in the workplace. \\
\hline s8 & 15 & $\begin{array}{l}\text { But when managed well, they can be helpful in promoting high performance, } \\
\text { creativity and innovation. }\end{array}$ \\
\hline s9 & 3 & Consequences of conflict \\
\hline $\mathrm{s} 10$ & 24 & $\begin{array}{l}\text { Whether or not conflict benefits people and organizations depends on two } \\
\text { factors: the intensity of the conflict and how well the conflict is managed. }\end{array}$ \\
\hline s11 & 18 & $\begin{array}{l}\text { The inverted ' } U \text { ' curve in Figure } 17.4 \text { shows that conflict of moderate } \\
\text { intensity can be good for performance. }\end{array}$ \\
\hline s12 & 15 & $\begin{array}{l}\text { This functional conflict, or constructive conflict, stimulates people to greater } \\
\text { work efforts, cooperation and creativity. }\end{array}$ \\
\hline s13 & 13 & $\begin{array}{l}\text { At very low or very high intensities dysfunctional conflict, or destructive } \\
\text { conflict, occurs. }\end{array}$ \\
\hline s14 & 23 & $\begin{array}{l}\text { Too much conflict interferes with other more task-relevant activities; too little } \\
\text { conflict may promote complacency and the loss of a creative, high- } \\
\text { performance edge. }\end{array}$ \\
\hline s15 & 12 & $\begin{array}{l}\text { Conflict is a disagreement over issues of substance and/or an emotional } \\
\text { antagonism. }\end{array}$ \\
\hline s16 & 13 & $\begin{array}{l}\text { Substantive conflicts involve disagreements over goals, resources, rewards, } \\
\text { policies, procedures and job assignments. }\end{array}$ \\
\hline s17 & 18 & $\begin{array}{l}\text { Emotional conflicts result from feelings of anger, distrust, dislike, fear, and } \\
\text { resentment as well as from personality clashes. }\end{array}$ \\
\hline s18 & 8 & Functional conflict is constructive and helps task performance. \\
\hline s19 & 8 & Dysfunctional conflict is destructive and hurts task performance. \\
\hline
\end{tabular}


When the recall mapping from Passages A6-A11 was analysed, several things became apparent and these are summarized below.

- Overall, differing sections of the text samples were recalled, suggesting that the Pacific Readers had responded to each text's own features. However it is important to note that some variation was found in the extent to which the Readers demonstrated this responsiveness to the text at hand or instead demonstrated their own predetermined and fixed approaches to all the texts they encountered.

- Information that was repeated in the text was usually recalled (e.g. A6: s6 \& s17 and A10: s13 \& s17).

- Definitions were generally recalled.

- Information with dates, names and key words from the title were usually recalled, even when this was not in main idea IU (e.g. A7: s8 \& s11, and A9: s13 \& s14).

- Specific example information tended to be recalled, but not always the main idea that it was exemplifying (e.g. A10: s14 \& s15 and A9: s8 \& s9).

- Main idea information with strong lexical signal words was usually recalled - with one noticeable exception in A11: s14.

- Main idea information and its explanations that were not well signposted with lexical clues were often not recalled (e.g.A8: s6-s10 and A6: s10). It was clear also that Readers were responsive to textual clues at the beginnings of sentences, and that the recognition and recall of main idea information did not always occur when the sentences did not contain clear signal words or that they were not in sentence initial position. 
Many of the patterns in the Recall of specific IU across the four Readers were most likely due to characteristics of the Text, such as the relative 'importance' of the ideas contained in the IU. The presence and absence of IU in the Recall may also be the result of selection and comprehension processes undertaken by the Reader (often as a result of these characteristics in the text). There are some features of this approach which could be interpreted as suggestive of a 'search and destroy' and scanning approach to the reading task. This is primarily an outcomes-focused and task-oriented means of text interaction, and one which may prove quite effective in some situations but may also have some limitation in higher education. However to label it purely as a 'surface' approach would overlook (and thus fail to activate and utilize) the strengths that do in fact exist within each Reader's reading persona and in their characteristic approaches to text.

\section{(8.3.3) Patterns of IU Omission in Free Recalls}

From the earlier Recall patterns it is clear that there were sections of text in the Course A text samples wherein the IU were not recalled by any of the Pacific Readers. While the kinds of elements that were not recalled did vary somewhat between text samples, Table 8.8 below records the patterns which emerged. 
Table 8.8: Textual elements not present in Pacific readers' IU free recall transcripts for passages A6-A11 in Course A

\begin{tabular}{|l|l|}
\hline Passages & Textual elements not present in students' immediate Free Recall \\
\hline $\begin{array}{l}\text { Passage } \\
\text { A6 }\end{array}$ & $\begin{array}{l}\text { - elements contained in lists (particularly the middle items of lists) } \\
\text { - some summary-type statements } \\
\text { - adverbial/descriptive elements }\end{array}$ \\
\hline Passage & $\begin{array}{l}\text { - descriptive elements } \\
\text { - elements in which an idea was being explained or expanded } \\
\text { - elements in which the sequential consequences of the main idea } \\
\text { point were being explained }\end{array}$ \\
\hline $\begin{array}{l}\text { Passage } \\
\text { A8 } \text { - rems of lists }\end{array}$ & $\begin{array}{l}\text { a great proportion of the Passage was not represented in the Recall. } \\
\text { - elements describing consequences } \\
\text { - items of lists } \\
\text { - main idea information from the central section of the text sample } \\
\text { - final sentences containing summary and consequence information }\end{array}$ \\
\hline $\begin{array}{l}\text { Passage } \\
\text { A9 }\end{array}$ & $\begin{array}{l}\text { - items in conjunction structures } \\
\text { - list items } \\
\text { - several pieces of main idea information (from the beginning, middle } \\
\text { and end of the Passage) } \\
\text { - descriptive elements (particularly those in list formation). }\end{array}$ \\
\hline $\begin{array}{l}\text { the material not recalled came from regular intervals in the text } \\
\text { - elements in conjunctions structure (with 'and') } \\
\text { - examples typed in brackets } \\
\text { - list items conjoined by conjunctions } \\
\text { - list items } \\
\text { - references to the Figure number } \\
\text { - a definition of an item previously defined in a slightly different way }\end{array}$ \\
$\begin{array}{l}\text { a proportion of the middle section of this text was not recalled by any } \\
\text { of the four students whose transcriptions allowed analysis. } \\
\text { - the title of the book referred to in the passage } \\
\text { - list items joined by conjunctions } \\
\text { - a reference to the Figure number } \\
\text { - descriptive elements describing the reason and manner } \\
\text { - four main idea sentences from the mid to end of the text (they } \\
\text { describe elements of the Figure) } \\
\text { - extra information inserted into a sentence and joined by conjunction } \\
\text { 'and' }\end{array}$ \\
\hline
\end{tabular}


The consistent patterns across four individual readings of each of the five selected text samples in Course A reveal that the following elements were regularly omitted from the verbal text Recalls of these Pacific Readers:

- list items (particularly mid list items)

- elements in conjunction structures

- elements whose function is to explain or extend main idea information

- reiteration/summary/evaluation elements

- references to Figures in the text

In addition to this, it was noted that a number of sections which contained main ideas and their discussion were also omitted in the recall of some texts. This may have reflected the style of writing utilized in these Passages, and/or their rhetorical structures.

Similarly to what the recall patterns showed, these omission patterns appear to reveal that, in the process of meaning-making, these Pacific students were utilizing strategies for the selection of certain material in the text for differing levels of processing - some of which would allow 'remembering' in the Free Recall. It is also apparent that certain syntactic elements were regularly categorized as not being central to the meaning of the sentence, so effort was either not directed to the remembering of these items, or it was directed unsuccessfully.

The omission of items in certain structures such as lists and conjunction structures is consistent with previous research by Kintsch and Keenan (1973, p.272) who found that "[p]ropositions appearing in the surface structure as modifiers were poorly recalled”. Likewise “...subordinate propositions were recalled better than propositions that were low in the hierarchies that constitute the sentence structures..." (Kintsch \& Keenan, 1973, p.273). 
Overall, however, these Pacific Readers were responsive to conventional signals of idea 'importance' in text, that they possessed a definite degree of sensitivity to the textual cues that indicate the relative centrality of ideas, and that they were using this awareness to selectively process text. Interviews also revealed that the Readers often possessed a predetermined understanding of what they believed to constitute 'important' and 'not important' information in a text, and while reading they were making executive decisions about the use of cognitive and attentional resources. The systems these Readers have utilized for directing and allocating attentional resources are not failsafe, but they do indicate the basis of an important skill set that they should be able to refine with the right guidance and sufficient opportunities to practice within the higher educational context. As these Readers mature in their academic reading abilities, it would usually be anticipated that they would move from the primary approach to reading as a selecting process for what to memorise and what not to, to a better understanding of reading as a communicative act (Abisamra, 2001).

These results are positive in that they reveal these Pacific Readers as cue sensitive, strategic, and able to readily identify main ideas in text in the first reading. It is also convincing evidence that, on the whole, these Pacific readers do posses general textual schema knowledge appropriate to this genre (i.e. they are familiar with the 'textbook' genre in Course A). What is not known however, is whether these Readers possess textual schema knowledge and genre awareness for the other types of texts encountered in higher education that differ considerably from the familiar 'textbook' format. This too would be interesting to observe in future research.

From personal experience assisting first year Pacific students I would agree with Johns (1995) that learning the "generalized organizational structure that is 
identified by and serves the communicative purposes of a discourse community" (Johns, 1995, p.279) is a major part of the student reader's challenge as they negotiate their way through the set readings of higher education, not only in the first year but throughout their study as they are confronted by new genres and forms. In terms of the pedagogical implications of this, Johns (1995) argues for spending more time explicitly working with ESL students on the "cultural and linguistic contexts" of texts as well as the styles that exist within disciplines and the purposes they serve. This would also be likely to be beneficial for native English speaking readers as well.

Whilst the present analysis operated at the broader and more semantically based 'Idea Unit' level, rather than the detailed grammatical level of 'propositions' employed in Kintsch and Keenan's (1973) study, it nevertheless shows that these Readers did have the ability to distinguish between the various hierarchies of information within sentences. This is a vital skill for efficient and effective academic reading in the tertiary environment. However, its success depends on the reasons for reading, the skill of the reader in making such decisions while reading, and the appropriateness of their own set of parameters that determine what they consider to be 'important'. These parameters tend to be defined by context, task and genre, and to respond to text appropriately one must necessarily possess a flexible set of parameters sensitive to the configuration of these factors in each reading scenario. Again, students may need extensive guided practice with a wide range of such texts to develop such flexibility. The likelihood of this occurring in the first year of study in the contexts observed seems small.

The data has also revealed that problems resulted when some of these Pacific Readers appeared to inflexibly apply certain set parameters and beliefs to all reading experiences, and were unresponsive to the nature and characteristics of 
the actual Text and Task at hand. An approach to addressing this situation is to work on developing 'strategic competence' to complement the language, skills and knowledge development that occurs in the first year of higher education. Further research is needed here too.

\section{(8.4) Post-task Interview Findings: What the Readers Themselves Reported about How They Read}

Discussion moves now from researcher's observations to a focus on the Readers' own self-reported processes. Academic reading is always purposeful, and whether explicitly known or not, it is driven either by an internal motivation or an external one. This reading purpose dictates reading practice, and thus, strategy use varies depending on what the goal of the activity is, "for example, reading for meaning demands different skills than reading for remembering (studying)" (Baker \& Brown, 2002, p.354) and so forth. We can therefore assume that each reader operates with some form of conscious or sub-conscious task representation, and that this determines their purpose/goal, which determines their strategy and skill use, which in turn prescribes the particular mechanisms the reader uses to make meaning in the dynamic Text-Task-Reader interplay of that particular reading scenario.

Immediately after completing each Free Recall task the Readers were asked to recount how they went about the task of 'reading to understand and remember' with each of the six FR text samples. The following themed commonalities across the Course A Pacific Readers' post-task interview data were found. 
Table 8.9: Pacific readers' common meaning-making and memory-related approaches to the Course A texts during the task of 'reading to understand and remember'

\begin{tabular}{|c|c|}
\hline $\begin{array}{l}\text { Readers' common meaning-making and memory-related } \\
\text { approaches }\end{array}$ & Utilized by: \\
\hline $\begin{array}{l}\text { Seeking the familiar, linking to prior learning, life and world } \\
\text { knowledge and experiences }\end{array}$ & $\begin{array}{l}\text { S9(F), } \\
\text { S4(M), } \\
\text { S13(PF), } \\
\text { S12(M) }\end{array}$ \\
\hline $\begin{array}{l}\text { Actively seeking relationships between and hierarchies of ideas in } \\
\text { texts. Demonstrating sensitivity to and exploitation of cohesive } \\
\text { devices in text. }\end{array}$ & $\begin{array}{l}\text { S9(F), } \\
\text { S4(M), } \\
\text { S13(PF), } \\
\text { S12(M) }\end{array}$ \\
\hline Orientation to noticing and memorizing 'facts and figures' in text & $\begin{array}{l}\text { S9(F), } \\
\text { S4(M), } \\
\text { S13(PF) }\end{array}$ \\
\hline $\begin{array}{l}\text { Sensitivity to unknown vocabulary and acquisition/extending of } \\
\text { vocabulary knowledge through encounters with text }\end{array}$ & $\begin{array}{l}\text { S9(F), S7(F), } \\
\text { S4(M), } \\
\text { S13(PF) }\end{array}$ \\
\hline $\begin{array}{l}\text { Selective and directed reading targeting beginnings and/or ends of } \\
\text { text to gain main ideas }\end{array}$ & $\begin{array}{l}\text { S9(F), S7(F), } \\
\text { S12(M) }\end{array}$ \\
\hline $\begin{array}{l}\text { Memorization (through repeated reading) as primary memory and } \\
\text { recall aid }\end{array}$ & $\begin{array}{l}\text { S9(F), S7(F), } \\
\text { S4(M) }\end{array}$ \\
\hline $\begin{array}{l}\text { Sensitivity to, being influenced by, and utilizing text layout and non- } \\
\text { linguistic features }\end{array}$ & $\begin{array}{l}\text { S13(PF), } \\
\text { S4(M), } \\
\text { S9(F), } \\
\text { S12(M), } \\
\text { S7(F) }\end{array}$ \\
\hline $\begin{array}{l}\text { Sensitivity to, being influenced by, and utilizing text's rhetorical } \\
\text { structure }\end{array}$ & $\begin{array}{l}\text { S13(PF), } \\
\text { S9(F), } \\
\text { S4(M), }\end{array}$ \\
\hline
\end{tabular}

This list above of most preferred and commonly utilized strategies and approaches for dealing with academic text generated in this study holds many clues to the ways in which these Pacific Readers view academic reading and the particular task of 'reading to understand and remember'. 
Contemporary reading comprehension models assert that the reader uses a combination of (1) background knowledge, (2) linguistic knowledge and (3) text knowledge to comprehend, and that meaningful comprehension cannot occur without all three (Pretorius, 2006). This study's findings clearly support this model. The most frequent meaning-making approaches documented amongst the case study Readers in Course A included active linking to prior knowledge, responses surrounding the lexis of the text, and conscious attention given to noticing and utilizing various linguistic and non-linguistic features of text.

Clear similarities also emerge when these results are compared with those carried out on readers from other ethnic and linguistic backgrounds. One such notable example is Kong's (2006) study which reports that Chinese adults in the USA for study utilized a variety of strategies for reading in English, and these were divided into two categories: (a) Text-initiated strategies: such as focusing on vocabulary, recognizing and exploiting the text structure, summarizing, utilizing pictures) and (b) Reader-initiated strategies: such as invoking prior knowledge, predicting, evaluating, monitoring, translating. Certain clear similarities (and of course some differences) can be seen between Kong's readers and the ones in this study.

In a study of critical thinking and belief systems Kemp (1963, p.322) proposed a list of factors thought to improve ability to engage critically with content. These included processes and thought patterns related to analysis, synthesis and evaluation, recognition and processing of relationships, flexibility, the evaluation of any conclusions drawn for their inclusivity and basicity, and adherence to the conditions given for the critical thinking task. Kemp's list of factors thought to decrease the ability to think critically about material read included: 
- ignoring, distorting or omitting ideas

- including additional words or ideas

- being rigid and nonadaptive

- failing to synthesize well

- incompleteness or absence of evaluation.

When Kemp's (1963) latter list is compared to the Pacific readers in this study some parallels emerge. The presence of error in Readers' transcript IUs was, consistent with Kemp's observations, frequently due to the omission of information, poor synthesis, or the insertion of extraneous ideas. Limitations in their FR transcripts revealed the general tendency not to evaluate, and that some of the Readers were notably more rigid in their responses to the text - as if following set formulaic patterns of response instead of responding to the text at hand (paralleling the other of Kemp's observed features associated with less critical engagement with content). Thus, it appears that all of the Course A Pacific Readers exhibit certain (if not all) of the characteristics thought to inhibit critical thinking. This is of particular note because of the centrality of 'critical reading' to the observed reading tasks and processes in the first year of university.

Thus, while possessing their own distinctive approaches, these first year Pacific Readers also demonstrated many of the characteristic approaches to text exhibited by other reader groups, sharing many strategy preferences with ESL readers, and this is interesting given that the majority of these Readers would be technically classified as English 'first' language speakers. Overall, these commonalities across different reader groups are in many ways also not surprising, since there is considerable support for the notion that "strategies relate to underlying cognitive processes (the way information is accessed and 
processed via language and thinking skills) and thus are not language specific" (Fraser, 1989, p.76).

\section{Chapter Summary}

This chapter has reported the findings on how Readers responded affectively to the texts in terms of their perceptions of Passage Interest and Passage Difficulty, and their success on the Free Recall task as measured by the percentage of Idea Units in their Recall. Overall it was found that Readers often responded to the same Passage in quite different ways, and that Interest and Difficulty perceptions clearly affected different Readers in different ways. This chapter's results show that the recurring themes of the importance of these Pacific Readers' affective responses to text appears to relate less to success on actual tasks and more to levels of overall motivation and persistence in academic reading.

Analysis of the outcomes and reported processes of the reading task via immediate Free Recall procedure, revealed the actual strategies and mechanisms by which the chosen academic reading Task was undertaken. Identification of the number, type and extent of Idea Units recalled when performing the task of 'reading to understand and remember' revealed that certain features of text were seen to influence meaning-making. There were also patterns in the Readers' personal approaches to the task that were likely to have been based on the internal task representations that they held for this basic academic reading task.

Having thus observed aggregate patterning across the group and the specific interaction between the features of the Text and those of the Reader, the next chapter considers the individual variation that the study revealed amongst the 
Course A Pacific Readers. Utilizing the framework for the holistic profiling of the Reader, the following chapter presents the holistic Reader profiles of two Course A Readers, S7(F) and S13(PF). 


\section{CHAPTER 9}

\section{THE INDIVIDUAL ACADEMIC READER PROFILES: WEAVING TOGETHER ALL THE STRANDS}

This chapter presents the individual Reader Profiles for Readers S7(F) and S13(PF) who were case study Pacific participants in Course A.

The chapter is presented in the following sections:

(9.1) Reader S7(F)'s Holistic Profile

(9.1.1) S7(F)'s Reading History (Salient Reading Memories)

(9.1.2) S7(F)'s Beliefs about Reading in the Discipline

(9.1.3) S7(F)'s Vocabulary Profile \& Factors Relating to Vocabulary

(9.1.4) S7(F)'s Reading Rate \& Factors Relating to Reading Rate

(9.1.5) S7(F)'s Self-reported Academic Reading Strategies and Attitudes

(9.1.6) S7(F)'s \% IU Recall \& Factors Relating to Recall

(9.1.7) S7(F)'s Perceptions of Interest \& Factors Relating to Interest

(9.1.8) S7(F)'s Perceptions of Difficulty \& Factors Relating to Difficulty

(9.1.9) How S7(F) Reported that she Interacted with the Texts during the FR Protocol.

(9.1.10) Weaving Together the Strands of S7(F)'s Profile

(9.2) Reader S13(PF)'s Holistic Profile

(9.2.1) S13(PF)'s Reading History (Salient Reading Memories)

(9.2.2) S13(PF)'s Beliefs about Reading in the Discipline

(9.2.3) S13(PF)'s Vocabulary Profile \& Factors Relating to Vocabulary

(9.2.4) S13(PF)'s Reading Rate \& Factors Relating to Reading Rate

(9.2.5) S13(PF)'s Self-reported Academic Reading Strategies and Attitudes

(9.2.6) S13(PF)'s \% IU Recall \& Factors Relating to Recall

(9.2.7) S13(PF)'s Perceptions of Interest \& Factors Relating to Interest

(9.2.8) S13(PF)'s Perceptions of Difficulty \& Factors Relating to Difficulty

(9.2.9) How S13(PF) Reported that she Interacted with the Texts during the FR Protocol. 
Although the Reader features have thus far been presented in separate sections for clarity, they should always be considered as inextricably intertwined. Mann (2000, p.301) describes the many "discrete "facts"' involved in reading research as "rather like the pieces of a mosaic which have been uncovered on an archeological dig. They can be classified according to colour or shape or texture, but unless an attempt is made to assemble them into some kind of picture they remain meaningless". In this study this 'assembly' of the pieces into a whole is the approach underlying the Reader Profiling framework, and the guiding metaphor to guide this process has been the woven Pandanus fibre mat. Each of the Pandanus strands used in the weaving is carefully selected, cut, handled and prepared. Although of value in themselves, the strands alone cannot provide a covering for the floor of the family's fale (house). They must be woven together, and when they are, a firm basis upon which decisions about planning and supporting their own (and future generations') academic literacy growth and development can be made in an informed and strategic manner.

What follows are summaries of the most salient features of the holistic academic reading profiles of two Course A Pacific case study Readers for whom complete profile data were collected in this study. From these summaries it is intended that the the value of the holistic framework for reader profiling may be seen at work and the unique nature of each Reader will be appreciated. 


\section{(9.1) Reader S7(F)'s Holistic Profile}

\section{(9.1.1) S7(F)'s Reading History (Salient Reading Memories)}

First language:

Parents:

Siblings:

Home languages:
Samoan and English

Mother is European, Father is Samoan. Was raised by her Grandmother for some period of her childhood. During these years she estimates that she spoke 25\% Samoan, 75\% English.

Speaks only English with siblings

99\% English, 1\% Samoan

S7(F)'s reading history interview reveals a background where some family members habitually read and some did not. Her mother read and had books but overall, her parents were not actively involved in or promoting of her reading experiences. However $\mathbf{S 7 ( F )}$ 's older sister was a clear reading role model in the home and remained a consistent role model throughout her childhood and early teens.

Always a high achiever in school S7(F) was promoted a class and reported doing well in most levels. In late primary school her main interest was sports, so reading was not an activity she had time for or interest in pursuing. Throughout her early to mid teens $\mathbf{S 7}(\mathbf{F})$ reported being aware of her sister reading and asking her sister to tell the stories to her. She preferred hearing about them or seeing the movie versions of popular books than reading them herself. Similarly she had enjoyed hearing teachers read to the class at earlier school levels.

Although a good reader, with a presumably good attitude to reading and herself as a reader, she reported only reading if she had to and if there was some form of motivation and clearly perceived 'payoff'. A period of renewed interest in reading was spurred in the later stages of high school by the need to write summaries and reports of novels set as compulsory reading. However 
with the advent of the internet and having her own laptop, her habits of reading were soon lost. Sporting activities again also took up much of her free time. S7(F) felt that her family and background had not had much effect on shaping her as a reader but rather that it was her school experiences that had had the greatest impact.

\section{(9.1.2) $S 7(F)$ 's Beliefs about Reading in the Discipline}

(Findings from End of Course Interview)

S7(F) believed that reading was important for understanding content in the Course, but that most of her learning actually occurred in tutorials when listening to presenters and to discussions. This is consistent with her reported preference for hearing text ideas told/read to her in her reading history. S7(F) reported that she did the set readings only when she perceived that there were negative consequences for not doing so. S7(F)'s experience was that the various lecturers for this joint-lectured course sent differing messages about the need to read before class, and thus $\mathbf{S 7}(\mathbf{F})$ varied in her engagement with the set text throughout the trimester. S7(F) knew that she needed to read and was, in theory, willing to do so. In practice, however, her motivation levels fluctuated daily and moved from initially high motivation to periods of very low motivation and back to high motivation towards the end of the Course.

S7(F)'s perception of the set text reveals that she had some knowledge of the textbook's reference features but seldom made use of them, however she believed that apart from being a little wordy and lengthy the set text was good

for memorizing from. Interestingly, $\mathbf{S 7}(\mathbf{F})$ did not naturally recognize any connections between the various chapters and her use of the textbook was geared primarily toward assessment completion. S7(F)'s perceptions about the authority of printed text precluded her from taking much authority over the 
content of text. She reported that she seldom explicitly thought of text as having an author, or of texts within the discipline having anything to do with each other, and so was quite limited in her author awareness and understanding of intertextuality. $\mathbf{S 7}(\mathbf{F})$ perceived the aim of the Course to be developing a core understanding of the content, theories and terminology of the discipline. At the time of the interview $\mathbf{S 7}(\mathbf{F})$ had, in hindsight, come to the conclusion that it was short-sightedness on her part that had caused her to lose sight of the goal. She believed she was fully capable of doing the readings and that she could have sustained her efforts over that period if she had been able to see the big picture.

S7(F) also reported a realization that her usual methods of reading were not serving her as well in higher education as they had done in high school and that something needed to change, but she was not sure what it was. Being involved in the study had caused S7(F) to think critically about herself as a reader for the first time. She expressed surprise in finding that other people read differently from her. As a result of our discussions during the study she felt that she would alter some of her approaches to reading and seek to learn more about how other successful readers accomplished their academic reading. S7(F) knew at the time of the interview that she had not passed this Course but was determined to try again the following trimester. She was aware that she would need to work very hard on sustaining her motivation levels.

\section{(9.1.3) S7(F)'s Vocabulary Profile \& Factors Relating to Vocabulary}

S7(F)'s knowledge of the Vocabulary levels and the Academic Vocabulary in the Levels Test was high, indicating a large vocabulary and good knowledge of the low frequency words. 


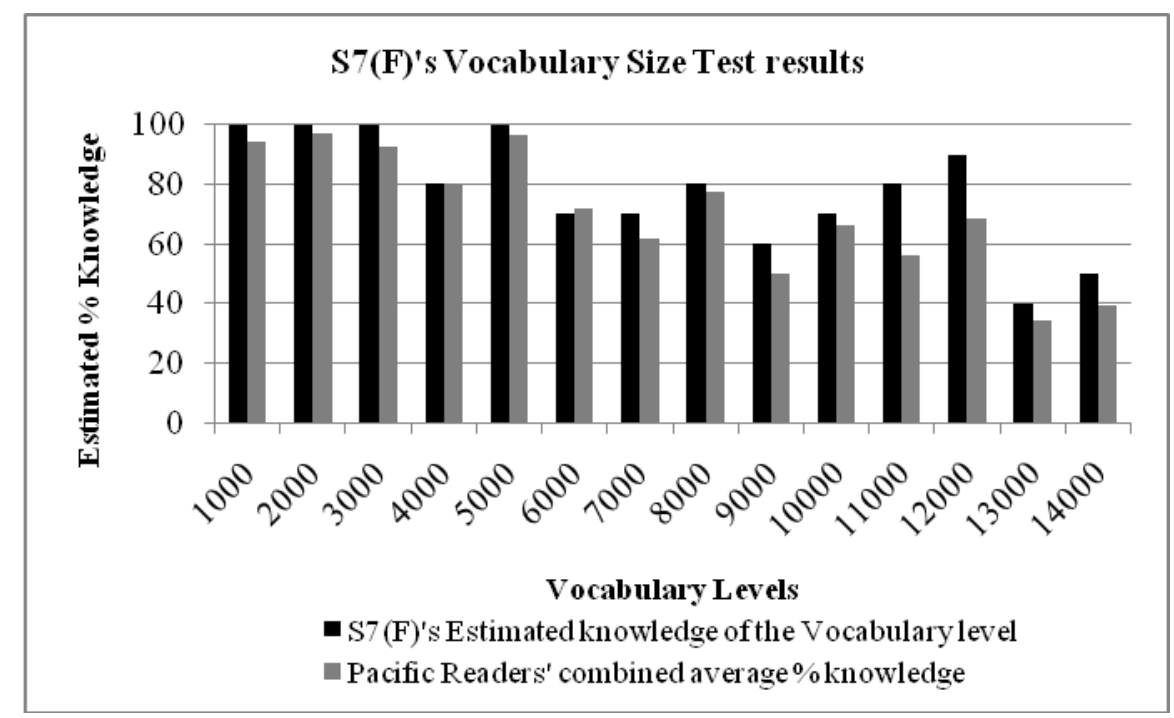

Figure 9.1: Reader S7(F)'s vocabulary size test results compared to the average for these Course A Pacific readers

S7(F)'s knowledge of the vocabulary levels was higher than the average of the Pacific Readers for all but two levels. Her estimated vocabulary size was roughly 10900 of the 14000 words in Levels 1-14, while the average of the Pacific Readers in Course A was 9500. S7(F)'s interviews revealed that her primary approach for dealing with unknown vocabulary was to guess from context, and that she seldom used other approaches or referred to reference material.

\section{(9.1.4) S7(F)'s Reading Rate \& Factors Relating to Rate}

S7(F)'s overall average Rate is about 11\% higher than the Readers' combined average. 
Table 9.1: Reader S7(F)'s reading rates for Course A passages compared to Course A Pacific readers' average rates

\begin{tabular}{|l|l|l|l|l|l|l|l|l|l|l|l|l|}
\hline & \multicolumn{1}{|l|}{ Course A Text Samples } & & & & & & & & & & & \\
& A1 & A2 & A3 & A4 & A5 & A6 & A7 & A8 & A9 & A10 & A11 & $\begin{array}{l}\text { Aver- } \\
\text { age } \\
\text { read- } \\
\text { ing } \\
\text { rate } \\
\text { for } \\
\text { stu- } \\
\text { dent }\end{array}$ \\
\hline S7(F) & 270 & 204 & 223 & 207 & 175 & 147 & 173 & 170 & 180 & 203 & 197 & $\begin{array}{l}195 \\
\text { wpm }\end{array}$ \\
\hline $\begin{array}{l}\text { AVERAGE } \\
\text { tate for } \\
\text { sample }\end{array}$ & $\begin{array}{l}205 \\
\text { wpm }\end{array}$ & $\begin{array}{l}172 \\
\text { wpm }\end{array}$ & $\begin{array}{l}166 \\
\text { wpm }\end{array}$ & $\begin{array}{l}173 \\
\text { wpm }\end{array}$ & $\begin{array}{l}169 \\
\text { wpm }\end{array}$ & $\begin{array}{l}160 \\
\text { wpm }\end{array}$ & $\begin{array}{l}173 \\
\text { wpm }\end{array}$ & $\begin{array}{l}158 \\
\text { wpm }\end{array}$ & $\begin{array}{l}193 \\
\text { wpm }\end{array}$ & $\begin{array}{l}184 \\
\text { wpm }\end{array}$ & $\begin{array}{l}176 \\
\text { wpm }\end{array}$ \\
$\begin{array}{l}175 \\
\text { wpm }\end{array}$ \\
\hline
\end{tabular}

The strong positive links between the percentages of K1 and Content words and S7(F)'s Reading Rate indicate that the percentages of these words in text are positively impacting her Reading Rate and are in themselves a good predictor of her Reading Rate. S7(F)'s rapid Reading Rate in Passages with high percentages of K1 words may be due to word familiarity increasing her confidence and causing her to read faster. On the other hand, since S7(F)'s perceptive responses reveal that $\mathrm{K} 1$ is also correlated negatively with Interest, it is thus possible that higher amounts of $\mathrm{K} 1$ may themselves hold little interest and cause her to skim read more, and faster, therefore taking less time to perform the task. The positive relationship between the percentage of Content words and Reading Rate is slightly harder to understand. Perhaps S7(F) is able to rapidly identify content words and utilize them to quickly build an understanding of text content.

Results suggested that Passages with lower IU Density were somewhat likely to be read faster by $\mathbf{S 7}(\mathbf{F})$, while Passages having greater SC (longer average sentence length) were read faster. Also of interest was that the Passages which were read at faster Rates were in fact those that she recalled the most IU 
from. This suggests that $\mathbf{S 7}(\mathbf{F})$ reads Passages with low conceptual density and high sentence length quickly and on the whole, the faster she reads these grammatically challenging but less conceptually dense texts, the better she is able to recall ideas.

\section{(9.1.5) $\quad S 7(F)$ 's Self-reported Academic Reading Strategies and Attitudes}

Findings from the Strategies and Habits Inventory.

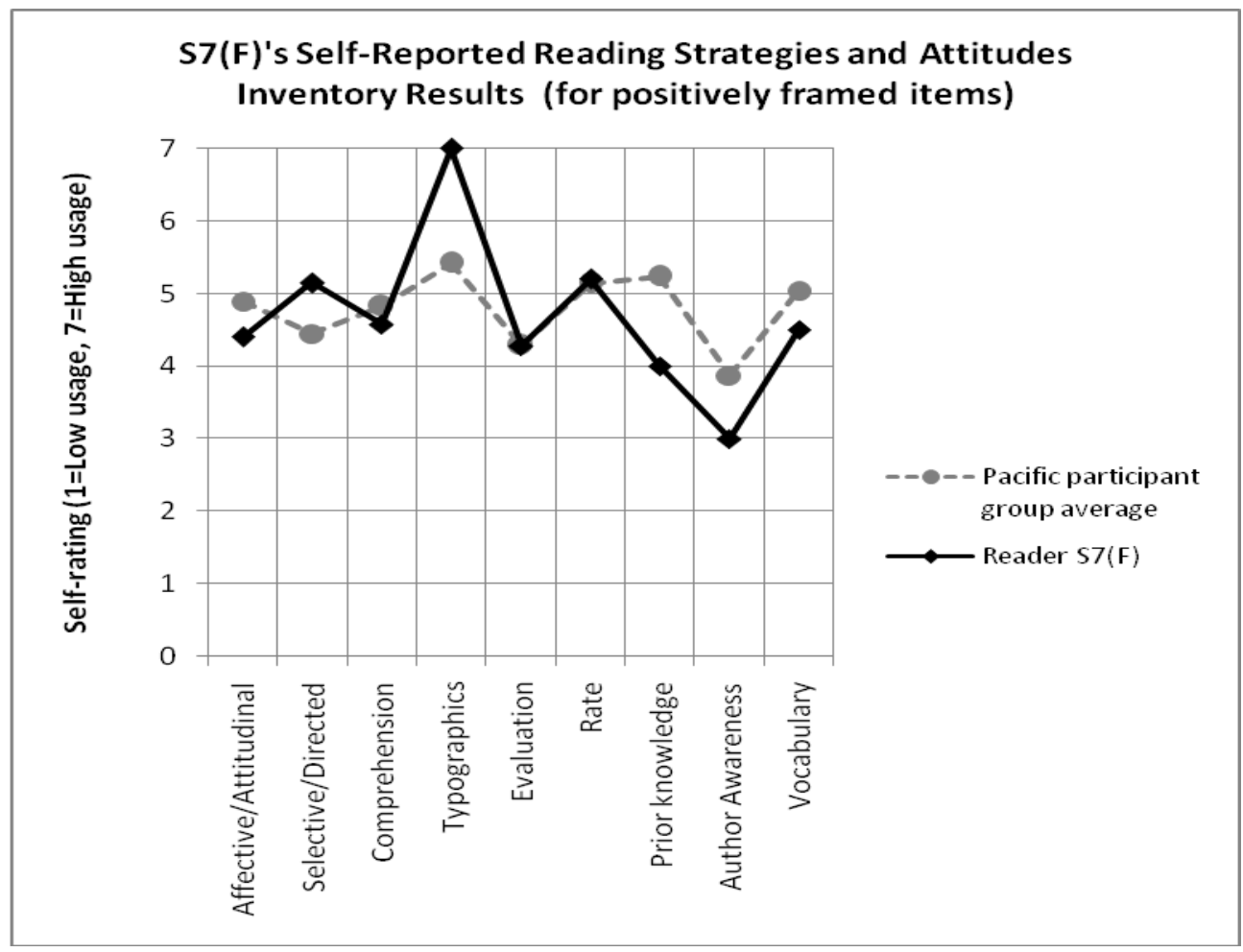

Figure 9.2: Reader S7(F)'s self-reported inventory results for positively framed items

S7(F)'s self-rating on the Inventory reveals a number of notable characteristics. The results indicate that $\mathbf{S 7}(\mathbf{F})$ reportedly utilizes a little below the average amounts of affective and attitudinal and the comprehension related 
strategies that are characteristic of 'good readers'. S7(F) also reports using well below average amounts of useful strategies for linking to prior knowledge, demonstrating author awareness and intertextuality and vocabulary related strategies. These present potential weaknesses in S7(F)'s academic reading approaches and would warrant developmental attention.

S7(F) reported using successful strategies for exploiting typographical and non-linguistic features in text to a considerably higher extent than the average Reader. If used appropriately these may prove to be a strong asset in her reading. However, not all academic texts provide the kind of non-linguistic features that this strategy set utilizes and such a heavy reliance on them may be fruitless (and frustrating) with such texts. Moreover, over-reliance on these may leave other strategic approaches to text underdeveloped. 


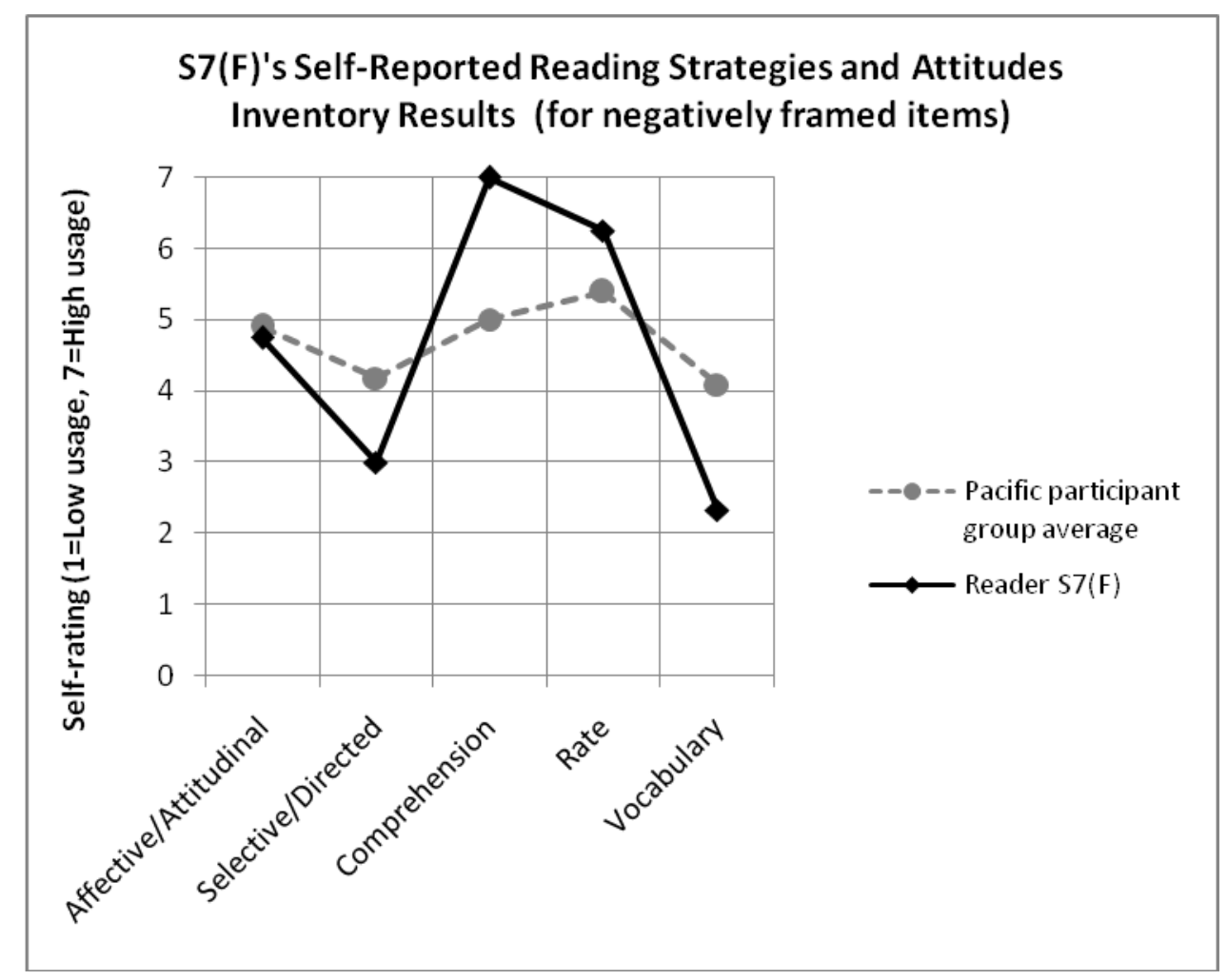

Figure 9.3: Reader S7(F)'s self-reported inventory results for negatively framed items

In terms of the strategies considered to be characteristic of less-skilled readers, S7(F) reports considerably higher usage of unhelpful comprehension and rate regulation strategies than the average Reader and this is consistent with findings from other parts of S7(F)'s profile that indicate that she values reading quickly, but may not have been utilising the most appropriate comprehension strategies for the new types of texts she was encountering in higher education. Low scores on the selective and directed and vocabulary scale indicate that she uses fewer of the strategic approaches associated with less skilled readers in these areas. This is a good feature of her profile and consistent with what other elements of her profile indicate - that she is already a reasonably selective Reader possessing a good vocabulary size. 
Looking at the ratings of individual items on the Inventory also reveals more about $\mathbf{S 7 ( F ) ' s ~ r e p o r t e d ~ s t r a t g i e s ~ a n d ~ a t t i t u d e s . ~}$

\section{Positively framed Statements rated "Always True"}

- marking or writing on the text itself while reading

- making use of tables, figures and pictures in text to aid understanding

- making use of typographical clues like bold type or italics

- making use of titles, headings and subheadings in text

\section{Positively framed Statements rated "Never True"}

- $\quad$ asking questions that one tries to answer as one reads the text

Negatively framed statements rated "Always True"

- concentrating on memorising a lot of what one reads

- 'saying' the words silently to oneself in one's head while reading

- $\quad$ while reading one re-reads numerous parts of text

Such high self-ratings for the strategies of subvocalisation and rereading are typically not found in readers with higher than average Reading Rates, since research and common sense tend to support the idea that these two are likely to slow Reading Rate down rather than to hasten it. It may be however, that S7(F) is comparatively over-generous in her self-ratings on these items.

\section{(9.1.6) $\quad S 7(F)$ 's \% IU Recall \& Factors Relating to Recall}

S7(F) achieved the following Recall rates in the Prompted and Free Recall: 
Table 9.2: Reader S7(F)'s prompted recall and free recall results for Course A passages

\begin{tabular}{|c|c|c|c|c|c|c|c|c|c|c|c|}
\hline \multirow[b]{2}{*}{ Passage } & \multicolumn{5}{|c|}{ Prompted Recall } & \multicolumn{6}{|c|}{ Free Recall } \\
\hline & A1 & A2 & A3 & A4 & A5 & A6 & A7 & A8 & A9 & A10 & A11 \\
\hline $\begin{array}{l}\text { Score / } \\
\% \text { IU } \\
\text { Recalled } \\
\text { by S7(F) }\end{array}$ & $47 \%$ & $43 \%$ & $30 \%$ & $53 \%$ & $61 \%$ & $24 \%$ & $29 \%$ & $19 \%$ & - & $35 \%$ & $29 \%$ \\
\hline
\end{tabular}

In terms of the impact of linguistic features of Text, like nearly all the other Readers, S7(F) clearly performed better on Prompted Recall than on Free Recall, suggesting that she actually took from text far more ideas than she could recall without prompts. This is interesting for a Reader whose profile repeatedly demonstrates a strong orientation to memorization during reading.

For S7(F), the results indicated that in texts with a higher percentage of Offlist words she recalled fewer Idea Units. In terms of Text Vocabulary characteristics, it appears that the Passages S7(F) was best able to recall were the ones that contained more of the high frequency and often encountered words (especially K1, Function words and Content words). In addition to her Recall being affected by Text Vocabulary features, it appeared that Reader S7(F) recalled less from Passages with high conceptual density (high IU Density). There did not however appear to be any systematic relationship between the grammatical complexity of the text (SC) and her Recall.

\section{(9.1.7) $\quad S 7(F)$ 's Perceptions of Interest \& Factors Relating to Interest}

The results indicate that some Text Vocabulary features do relate sytematically to S7(F)'s perceptions of Passage Interest, particularly Lexical Density (the density of Content words). On the other hand for S7(F), IU 
Density did not appear to relate in any way to perceptions of Interest but SC did slightly. It is possible that low Interest Passages engender in S7(F) varying levels of engagement with text, and that this is what results in the varying Recall success levels for texts of the same Interest rating. Overall however, and somewhat unusually, there does appear to be a negative relationship between Interest and Recall. It may be possible that Interest in a Passage's content may serve to 'distract' S7(F) from the task of memorizing content, and thus account for the reduced Recall success for Interesting Passages. However this requires more investigation.

\section{(9.1.8) S7(F)'s Difficulty Perceptions \& Factors Relating to Passage Difficulty}

For S7(F) the Passages with higher IU Density and the highest SC were likely to be experienced as being more Difficult. This indicates that these linguistic features of text did have an impact on S7(F)'s perceptions of Difficulty even though they did not appear to relate to Interest very much.

It is possible that $\mathbf{S 7 ( F ) ' s ~ g r e a t e r ~ s u c c e s s ~ i n ~ R e c a l l ~ f o r ~ t h e ~ P a s s a g e s ~ s h e ~}$ perceived as most Difficult has something to do with her self-reported rating as the highest of all the Readers in the use of selective/directed reading strategies and in the purposeful and useful exploitation of typographical features in text to aid her reading. It may be that when $\mathbf{S 7}(\mathbf{F})$ perceives texts to be increasingly Difficult she calls into play a very strategic approach to reading, relying at a surface level on typographical features and her ability to effectively scan text for main idea information and memorize it for later Recall.

In terms of vocabulary features, it appeared that for Reader S7(F), texts with higher proportions of the Off-List and Function words, the Number of Word Types, and the Type-Token Ratio all coincided with increased perceptions of 295 
Vocabulary Difficulty in Passages and were perceived as having more Difficult Vocabulary.

The overall combination of low Interest and high Difficulty in these Course A Passages is not a promising one, and is likely to relate to both intellectual and affective issues. $\mathbf{S 7 ( F ) ' s ~ p r o f i l e ~ r e p e a t e d l y ~ s h o w s ~ t h a t ~ t h i s ~ i s ~ s o , ~ a n d ~ t h e ~ l o w ~}$ and fluctuating motivation levels $\mathbf{S 7}(\mathbf{F})$ reported during the term are likely to have been linked to this.

\section{(9.1.9) How S7(F) Reported that she Interacted with the Texts during the FR Protocol}

The results suggest that $\mathbf{S 7}(\mathbf{F})$ makes meaning during 'reading to understand and remember' by utilizing the following approaches:

\section{S7(F)'s main meaning-making strategies with academic text in}

\section{Course A}

- Seeking to summarise sections of ideas in text, chunking them, but whose summaries did not always accurately maintain the sense of the original text. Grouping of ideas in recall follows the grouping of ideas within the Passage.

- Paying attention to diagrams and recall of diagram in relevant texts.

- Being strongly oriented to memorizing.

- Practising some linking to prior knowledge but very seldom were text ideas related to her own world knowledge or prior learning, both within the course and between it and prior or concurrent learning. Some attempts to link indicated that meanings and concepts were being extended in their use beyond her prior understanding.

- Seldom inserting own responses or evaluation into her recall, suggesting that these responses to text do not frequently occur in her task completion.

- Demonstrating sensitivity and alertness to new and unknown vocabulary. She reported learning a new term while completing the task. Her primary strategy was to guess the meaning of unknown words by breaking them into parts.

- Accurately identifying key terms but often recalling them in isolation (detached from the meaning to which they were attached in the text). 
- Frequently recalling verbatim units of up to six words in text, suggesting text is primarily stored for memory in linguistic units.

- Attempting to recall information according to its hierarchy in the text but some terms from lower on the hierarchy are presented as belonging to higher levels.

- On one occasion recalling a key term inaccurately due to 'colouring' of that word by a commonly known collocation in the Reader's general knowledge. This collocation included one of the key text terms.

- Referring to the text as an object and ideas being contained in it and evidence of a level of distance and objectivity from text.

- Committing ideas to memory from regular intervals spanning the whole text. This would suggest a focus on the perceived task of recalling facts from throughout the text rather than on gist-seeking.

- Reported regularly checking her understanding of the text, adopting a meaning-focused approach and utilizing comprehension monitoring.

- Being sensitive to and able to exploit textual clues and cues.

- Initially responding to meaning breakdowns by slowing reading down and re-reading. If that failed then she frequently resorted to skim reading rather than continuing close reading. Her understanding of some sections of text was impacted negatively by perceived lack of understanding in the preceding section of text.

- Being clearly impacted by her affective responses to text and the task of reading. Appeared to form an impression of the text within the first few sentences. This impression often determined the effort and persistence in meaning making utilized in reading the text.

- Possessing the desire to 'focus' and maintain efforts to make meaning fluctuated notably between passages and sometimes within Passages.

- Usually accessing the reference features of text and skim reading them after the Passage itself was read. Diagrams were accessed at the point in the text to which they were referred in the text, suggesting that they were incorporated as part of the meaning-making process.

- Predominantly utilizing the memory strategy of mentally repeating and rephrasing key ideas and terms. Frequently she would pause and test her recall of the definitions to gauge whether she had memorized it.

- Never making any comments that reflected her own strategy use or making any attempts to attribute her approach. Possibly indicating less meta-cognitive involvement in this task.

- Being influenced by the task, so that usual re-reading and highlighting did not occur to the extent it may have under 'normal' conditions.

- Being spurred to re-read when faced by conceptual density. 
- Being sensitive to stylistic features of text, and especially vocabulary choice and usage. S7(F)'s enjoyment of a text could also be affected by stylistic features.

- Being sensitive to her own rate regulation and regularly reporting conscious rate regulation within passages.

From these observations it became possible to piece together an understanding of S7(F)'s unique task representation. For S7(F), success in 'reading to understand and remember' means:

- recalling IU from regular intervals throughout the text (from beginning to end, regardless perhaps of the relative 'importance' of these ideas) and utilizing many of the author's original vocabulary and phrasing.

- completing the task quickly.

It appears that for $\mathbf{S 7}(\mathbf{F})$ success in 'reading to understand and remember' is demonstrated by the quantity and coverage of IU remembered, and this outcome is best achieved by memorization, and an important indicator of task success is rapid completion. S7(F)'s approach could be likened to a vacuum cleaner, which sucks up ideas uniformly across the text with speed and relative effectiveness. She stores these and is able to unload them when requested, mixed together and a little worse for wear, but still fairly much in the same shape as they were when sucked up.

\section{(9.1.10) Weaving Together the Strands of S7(F)'s Profile}

\section{Recommended Academic Reading Development for S7(F)}

Based on what S7(F)'s holistic Reader profile has revealed about her academic reading strengths and weaknesses the following is recommended for $\mathbf{S 7 ( F ) ' s ~}$ academic reading development for context-appropriate academic reading in Course A:

- Although she already possesses a large vocabulary, developing a wide range of useful strategies for dealing with unknown vocabulary in text 
will assist $\mathbf{S 7 ( F )}$ when morphology and context do not yield clues to word meaning. This may be particularly useful for technical vocabulary (OffList words), which these results show are associated with less success in Recall.

- S7(F) should be encouraged to become more familiar with the set texts, the genres they represent, and what features they may have for her to exploit for reading success.

- Encouraging S7(F) to consistently utilize strategies for linking to prior knowledge should aid her understanding and recall and make the task meaningful instead of mechanical.

- Developing more metacognitive control over comprehension-related strategies is necessary to ensure that the most appropriate ones are utilised at the right time.

- S7(F) may benefit from strategies for getting more out of text in the first reading to reduce the need for reliance on repeated re-reading of text.

- Self questioning (asking questions that one tries to answer as one reads the text) is a useful strategy $\mathbf{S 7 ( F )}$ may benefit from utilizing occasionally. It may even assist her to maintain interest and focus in less interesting texts.

- S7(F) may need assistance to monitor her use of the Reading Rate regulation strategies normally associated with less successful readers. 
○ Subvocalisation ('saying' the words silently to oneself in one's head while reading) is typically thought to have detrimental effects on reading rate and occasionally on comprehension, but further investigation is needed into the role it plays in $\mathbf{S 7 ( F ) ' s ~ r e a d i n g , ~ a s ~ i t ~ d o e s ~ n o t ~ a p p e a r ~ t o ~ s l o w ~ h e r ~}$ reading down and may in fact aid her comprehension as she has a strong orientation to 'hearing' text. Further investigation of this is necessary and S7(F) needs to become more consciously aware of her own reading processes such as this one to monitor its effective use.

- Guided practice in developing author awareness and a sense of intertextuality within the discipline will be crucial to $\mathbf{S 7}(\mathbf{F})$ progressing higher in her studies. S7(F) will also need to come to understand that her assessment and task focus and her orientation to reading as a 'performance' are associated with more shallow reading than is expected in higher education. With proper guided practice $\mathbf{S 7 ( F )}$ will be able gain the confidence and expertise to engage in critical reading.

- S7(F) would benefit from assistance with consideration of the role of memorization in her higher education studies.

○ S7(F) should develop and utilise more of the positive affective and attitudinal responses to text that are characteristic of 'good readers', particularly in relation to motivation for reading and engagement with text. She may benefit from the appointment of an academic mentor (similar to her sister's role in the home) to model and aid her developing awareness of critical and evaluative responses to text. 


\section{(9.2) Reader S13(PF)'s Holistic Profile}

\section{(9.2.1) S13(PF)'s Background and Reading History (Salient Reading Memories)}

First language: $\quad$ Samoan.

Parents:

Parents born in Samoa.

Siblings:

Has siblings and they converse in Samoan.

Home languages: Her parents had gone to another Pacific nation as missionaries and several of the children were born there. She received all her primary and secondary education in Samoa, she spoke $100 \%$ Samoan language in the home.

S13(PF)'s salient reading memories point to a background that was not rich in print material but where she did have several significant reading role models in the home. As well as observing both parents engaging in reading activities she also had the opportunity to read bilingual material and had several teachers who encouraged her to develop her English language and literacy profiency. S13(PF) had an internal motivation to read which was led mostly by her curiosity to know about other parts of the world, and it appears that S13(PF) has always had a positive attitude towards reading, despite reporting not being consciously aware of its importance until she reached higher education.

\section{(9.2.2) S13(PF)'s Beliefs about Reading in the Discipline}

(Findings from End of Course Interview)

S13(PF) liked the textbook, especially its graphics, and particularly enjoyed the case studies for explanation of points since they were like stories and she reported that she enjoyed learning through stories of real people and things. She was limited however in her awareness of the textbook's reference and self-help features, and like all other Readers, her main use of the set text was for assessment-related purposes. 
S13(PF) felt that it was not entirely necessary to read before lectures as Lecturers generally presented the reading material and one could avoid attention and questioning by sitting at the back of the class. She did however make an effort to read before tutorials since she enjoyed participating in discussions. S13(PF) felt that Lecturers made their reading expectations clear but that they did not model reading for students or give them guidance in how to do it.

S13(PF) felt that a students' role was to learn concepts and apply them. She reported that she did not engage in a critical way with texts, and did not feel that she had any authority to question what was written in the texts she read at university. S13(PF) had not explicitly thought about the set texts as having authors.

S13(PF) felt that she was committed to her reading in this Course, but that because it was not a major she did not read beyond the set texts. Her most useful strategy for academic reading was to read the text aloud to herself. She had however learnt some useful ways to interact with text from learning advisers, lecturers and tutors whom she had approached for help in her study. For unknown vocabulary she would refer to reference material, learn the meaning then practice using the word in context.

S13(PF) believed that her cultural background did influence her and other Pacific people's attitudes to text and reading. She had come to feel that reading was an important tool for personal betterment. Looking back at the time of the interview, S13(PF) felt that it would have been good to read more in this Course. She felt that she had not done well in the Course. After initially narrowly failing the exam she applied for a recount and eventually 
passed the Course. The best result for S13(PF) from being involved in this study was a stronger drive toward meaning-focused reading.

\section{(9.2.3) S13(PF)'s Vocabulary Profile \& Factors Relating to Vocabulary}

The results of S13(PF)'s vocabulary measures indicate that she knows less than the average of the Pacific Readers in all but one of the 14 Levels and knows about $6 \%$ less of the UWL than the average of her peers in this study. S13(PF)'s estimated knowledge was about $77 \%$ of the Academic Vocabulary in the UWL, while the average was $83 \%$.

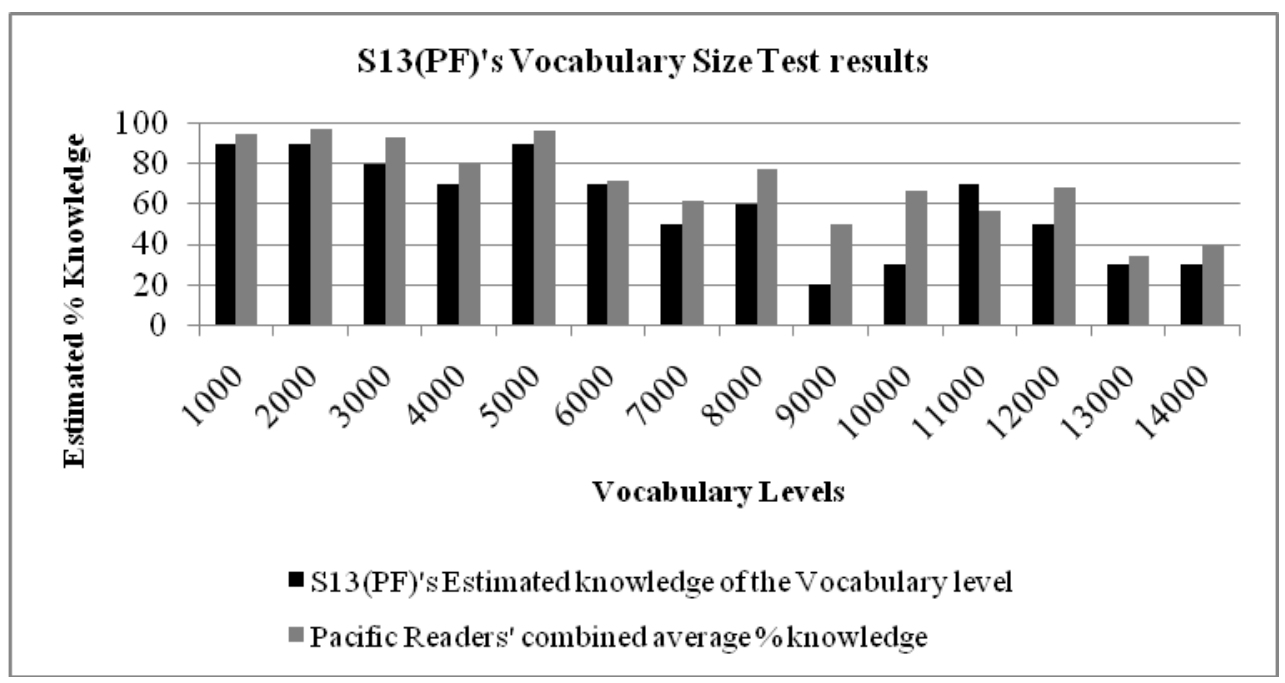

Figure 9.4: Reader S13(PF)'s vocabulary size test results compared to the average for these Course A Pacific readers

Her total vocabulary size on the 14 Levels was estimated at 8300 of the 14000 words. What is important to note is that $\mathbf{S 1 3}(\mathbf{P F})$ not only demonstrates lower knowledge of the less frequent vocabulary items but also demonstrates limitations in her knowledge of levels K1-K3. These limitations in general vocabulary are likely to present a considerable challenge when combined with 
the load of technical and specialist and academic words that these academic texts present.

Results reveal that the Passages S13(PF) recalled the most from were those with more Function words, and the Passages that she recalled the least from were those with a higher density of Content words. This finding varies quite a lot from other Readers in this study and may reflect her characteristic of being the only reader of the case study Course A Readers to be truly classified as an ESL reader.

The results of investigation of the relationship between Text Vocabulary and S13(PF)'s perceptions of text Vocabulary Difficulty suggest that when S13(PF) responds to text as having difficult vocabulary in it, she may be primarily responding to the presence of $\mathrm{K} 2$, AWL, Off-List items, the relative number of times certain words are repeated in the text, and the density of Content words.

\section{(9.2.4) S13(PF)'s Reading Rate \& Factors Relating to Rate}

Although S13(PF)'s Reading Rate fluctuated from text to text, her average Reading Rate was about $6 \%$ slower than the average Rate for these Pacific Readers in Course A. 
Table 9.3: Reader S13(PF)'s reading rates for Course A passages compared to Course A Pacific readers' average rates

\begin{tabular}{|c|c|c|c|c|c|c|c|c|c|c|c|c|}
\hline & \multicolumn{11}{|c|}{ Course A Text Samples } & \\
\hline & A1 & $\mathrm{A} 2$ & A3 & A4 & A5 & A6 & A7 & A8 & A9 & A10 & A11 & $\begin{array}{l}\text { Average } \\
\text { reading } \\
\text { rate for } \\
\text { student }\end{array}$ \\
\hline $\begin{array}{l}\text { S13 } \\
\text { (PF) }\end{array}$ & $\begin{array}{l}175 \\
\text { wpm }\end{array}$ & $\begin{array}{l}137 \\
\text { wpm }\end{array}$ & $\begin{array}{l}133 \\
\text { wpm }\end{array}$ & $\begin{array}{l}187 \\
\text { wpm }\end{array}$ & $\begin{array}{l}164 \\
\text { wpm }\end{array}$ & $\begin{array}{l}156 \\
\text { wpm }\end{array}$ & $\begin{array}{l}171 \\
\text { wpm }\end{array}$ & $\begin{array}{l}124 \\
\text { wpm }\end{array}$ & $\begin{array}{l}199 \\
\text { wpm }\end{array}$ & $\begin{array}{l}194 \\
\text { wpm } \\
\end{array}$ & $\begin{array}{l}169 \\
\text { wpm }\end{array}$ & $164 \mathrm{wpm}$ \\
\hline $\begin{array}{l}\text { AVER- } \\
\text { AGE } \\
\text { Rate for } \\
\text { text } \\
\text { sample }\end{array}$ & $\begin{array}{l}205 \\
\text { wpm }\end{array}$ & $\begin{array}{l}172 \\
\text { wpm }\end{array}$ & $\begin{array}{l}166 \\
\text { wpm }\end{array}$ & $\begin{array}{l}173 \\
\text { wpm }\end{array}$ & $\begin{array}{l}169 \\
\text { wpm }\end{array}$ & $\begin{array}{l}160 \\
\text { wpm }\end{array}$ & $\begin{array}{l}173 \\
\text { wpm }\end{array}$ & $\begin{array}{l}158 \\
\text { wpm }\end{array}$ & $\begin{array}{l}193 \\
\text { wpm }\end{array}$ & $\begin{array}{l}184 \\
\text { wpm }\end{array}$ & $\begin{array}{l}176 \\
\text { wpm }\end{array}$ & $175 \mathrm{wpm}$ \\
\hline
\end{tabular}

The strong negative relationship between the percentage of Off-List words and her Reading Rate shows that these technical, specialist and academic words had a considerably negative impact on S13(PF)'s Reading Rate, slowing it down markedly. It can be surmised that this is a natural result of limitations that were observed in her vocabulary profile. It may also indicate that she is using time-consuming strategies for dealing with the load of technical and specialist academic vocabulary that might comprise the majority of the Off-list words, and that this is slowing her Rate of task completion.

For S13(PF) the Passages with high IU Density and SC were those that she read the slowest. Overall however, there was no systematic relationship between S13(PF)'s Rate of Reading and her Recall of IU from texts, so these fluctuations in Rate did not appear to affect Task success.

\section{(9.2.5) S13(PF)'s Self-Reported Academic Reading Strategies and Attitudes}

S13(PF)'s self ratings on the Inventory reveal more about her unique reading persona. 


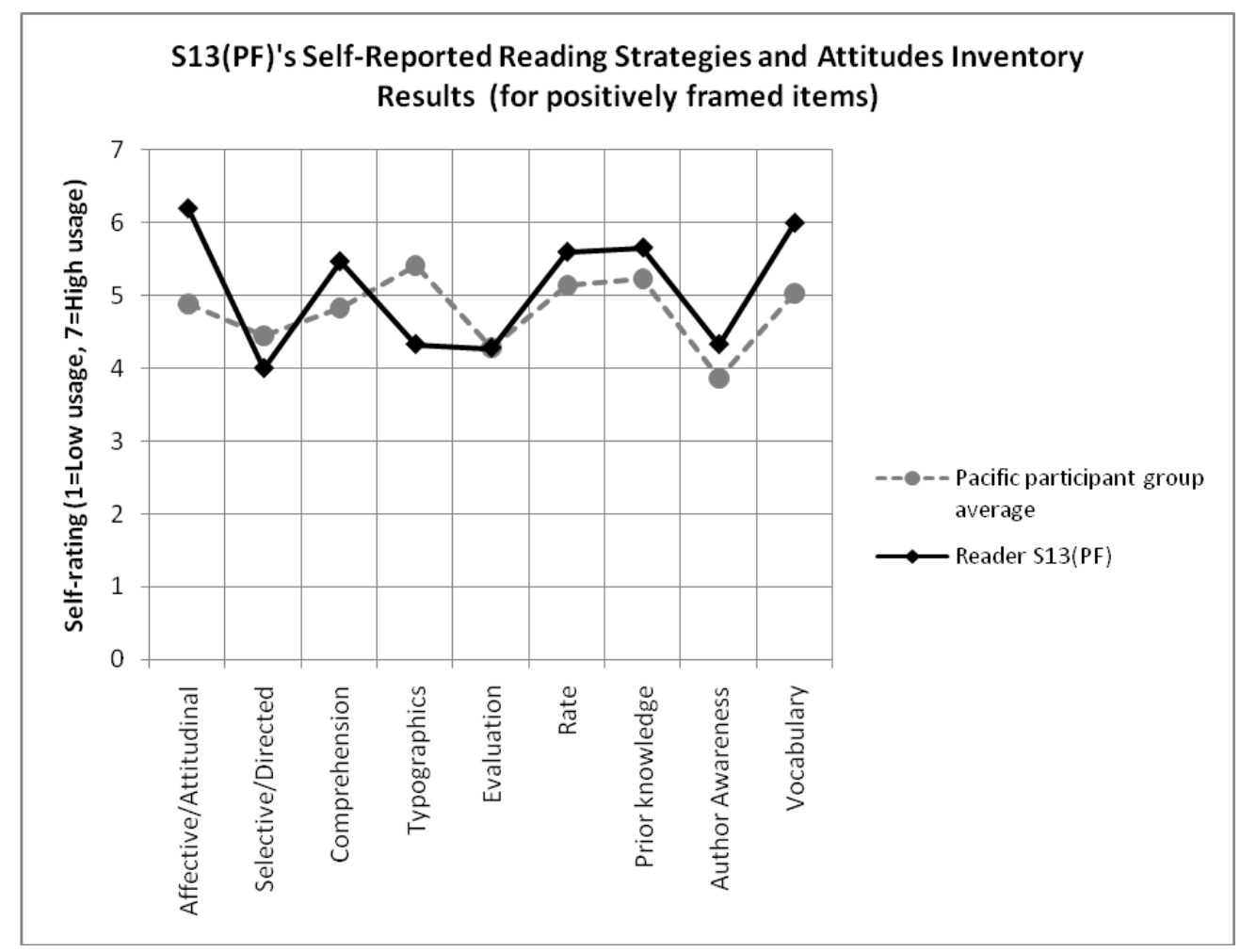

Figure 9.5: Reader S13(PF)'s self-reported inventory results for positively framed items

The results indicate that $\mathbf{S 1 3}(\mathbf{P F})$ reportedly utilises above average amounts of the useful strategies related to comprehension, reading rate regulation, utilisation of pior knowledge, author awareness and vocabulary. Furthermore, S13(PF) demonstrates well above average of the attitudinal characteristics of successful readers than the average for this group. This is a particular strength for Reader S13(PF). The two areas in which she may require development are the use of selective and directed strategic reading and the successful strategies for utilizing and exploiting text's typographical features. 


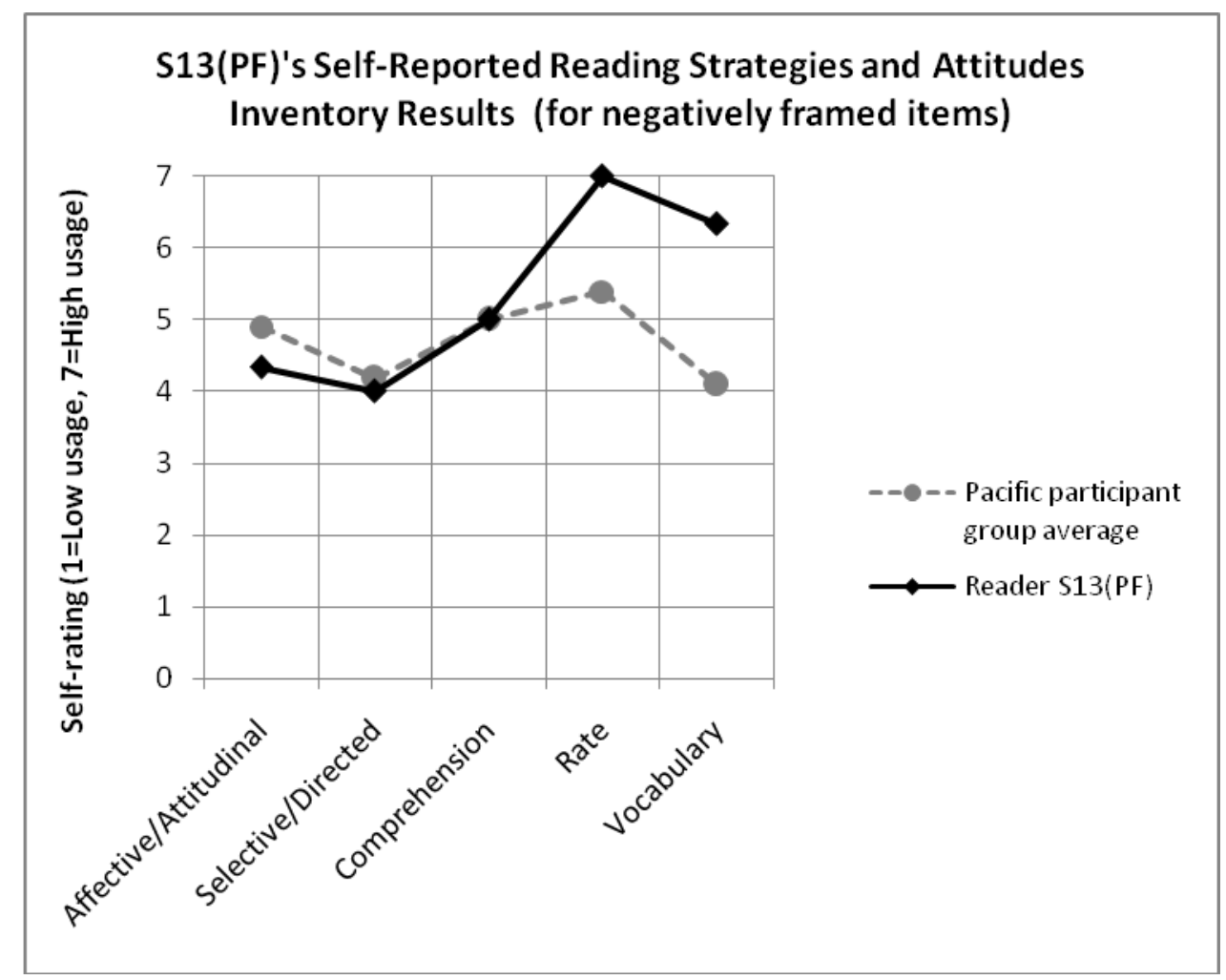

Figure 9.6: Reader S13(PF)'s self-reported inventory results for negatively framed items

In terms of the strategies that are reported by literature to be characteristic of less skilled readers, $\mathbf{S 1 3 ( P F )}$ appears to demonstrate roughly average amounts of these except for reading rate regulation and vocabulary strategies. It is these that would be likely to need adressing in her profile. It is worth noting though, that S13(PF) displays slightly less than average of the negative affective and attitudinal characteristics and this is characteristic of her positive attitude to reading and study.

The individual items in the Inventory that were rated high or low also reveal more about $\mathbf{S 1 3}(\mathbf{P F})$ as a reader: 


\section{Positively framed Statements rated "Always True"}

- liking books that challenge one and provide explanations which go beyond the

- lectures and seminars

- doing academic readings is a satisfying and productive experience

- being motivated to read by interest

- before reading, making sure that one is clear about one's purpose for reading

- while reading one is actively trying to stay focussed on what one is reading

- underlining or circling information in the text while one reads

- while reading one tries to elaborate on the ideas in the text

- while reading one tries to visualise or picture the ideas in one's head

- discussing one's reading with others

- adjusting one's reading speed according to what one is reading

- trying to relate the ideas in text to what one has already learnt in that course

- while reading trying to notice and learn new words/language items

\section{Positively framed Statements rated "Never True"}

- deciding what to read closely and what to ignore

Negatively framed statements rated "Always True"

- being motivated to read by fear of failure

- $\quad$ liking to read text aloud when reading

- 'saying' the words silently to oneself in one's head while one reads

- while reading one frequently re-reads parts of text

- reading word by word (one word at a time)

- looking up most of the unknown words one comes across while reading

- While reading one translates words or ideas into one's first or other language/s to help one understand

S13(PF)'s high self rating on the items relating to author awareness and positive rate regulation are however not consistent with my observations of her interactions with academic text in the Free Recall task and in general in Course A, or her interview data. This suggests that she may perceive herself as possessing and utilising more of the 'good reader' characteristics than she may in fact possess or have demonstrated in the FR Task. However her self308 
ratings on the use of less than average amounts of selective reading strategies and less than average utilisation of typographical features are very consistent with my observations of her reading, and present a definite area for development.

S13(PF)'s self ratings on the negatively framed items indicate that addressing less constructive rate regulation and vocabulary strategies would benefit S13(PF)'s reading. The negatively framed items rated 'always true' are likely to require developmental attention. These include motivational issues, subvocalisation and reading aloud (which impacts negatively on reading efficiency), reading fluency (in terms of fixation points on the page) and timeconsuming vocabulary strategies.

\section{(9.2.6) S13(PF)'s \% IU Recall \& Factors Relating to Recall}

S13(PF)'s overall success in IU Recall was as shown below:

Table 9.4: Reader S13(PF)'s prompted recall and free recall results for Course A passages

\begin{tabular}{|c|c|c|c|c|c|c|c|c|c|c|c|}
\hline & \multicolumn{5}{|c|}{ Prompted Recall } & \multicolumn{6}{|c|}{ Free Recall } \\
\hline Passage & A1 & A2 & A3 & A4 & A5 & A6 & A7 & A8 & A9 & A10 & $\begin{array}{l}\text { A1 } \\
1\end{array}$ \\
\hline $\begin{array}{l}\text { Score / } \\
\% \text { IU } \\
\text { Recalle } \\
\text { d by } \\
\text { S13(PF) }\end{array}$ & $\begin{array}{l}47 \\
\%\end{array}$ & $\begin{array}{l}33 \\
\%\end{array}$ & $\begin{array}{l}38 \\
\%\end{array}$ & $\begin{array}{l}28 \\
\%\end{array}$ & $\begin{array}{l}45 \\
\%\end{array}$ & $\begin{array}{l}7 \\
\%\end{array}$ & $\begin{array}{l}29 \\
\%\end{array}$ & $\begin{array}{l}19 \\
\%\end{array}$ & $\begin{array}{l}17 \\
\%\end{array}$ & $\begin{array}{l}15 \\
\%\end{array}$ & $6 \%$ \\
\hline
\end{tabular}

Like all other Readers, S13(PF) performed better on the Prompted than the Free Recall. Her average \% IU Recall for the FR task was $16 \%$. Comparatively, this is a very low average amount of IU Recall. 
S13(PF)'s results suggest that the Passages of both higher conceptual and syntactic complexity were those that $\mathbf{S 1 3}(\mathbf{P F})$ was able to recall the most Idea Units from. This is a somewhat unexpected result, since one would anticipate that less conceptual density and shorter sentences would facilitate greater comprehension and recall by an ESL reader, rather than the opposite being true. More investigation is needed to understand these aspects of S13(PF)'s profile.

\section{(9.2.7) S13(PF)'s Perceptions of Interest \& Factors Relating to Passage Interest}

The correlation between Text Vocabulary features and S13(PF)'s Interest levels indicate that there is some tendency for the Texts that $\mathbf{S 1 3}(\mathbf{P F})$ finds more Interesting to have higher percentages of Off-List and Function words. This is a similar result to some of the other Readers in this study and suggests that the more 'specialised' or 'technical' a Passage was, the more it was perceived as being Interesting. On the whole however, IU Density and SC in Text did not appear to impact S13(PF)'s perceptions of text Interest.

\section{(9.2.8) S13(PF)'s Difficulty Perceptions \& Factors Relating to Passage Difficulty}

In terms of Difficulty, S13(PF)'s Difficulty ratings were higher for the Course A Passages that had higher percentages of K2, AWL, Off-List, and greater Numbers of Word Types, Type-Token Ratios, and Lexical Density. IU Density was a good predictor of how Difficult the Passage would appear to her, unlike her results for Interest. For S13(PF), overall, both Interest and Difficulty perceptions had a small positive relationship with her Recall. 
Drawing together all the analyses, it appears that S13(PF) makes meaning from academic text in the task of 'reading to understand and remember' by utilizing the following processes:

\section{S13(PF)'s main meaning-making strategies with academic text in Course A}

- Predominantly recalling information verbatim or in words from the text, suggesting that she stores this information primarily in linguistic units.

- Seeing the text as an object having parts in a set order. Ideas were recalled in relation to their position in the structure of the text.

- Gauging the familiarity of ideas from the title and first few sentences.

- Forming a partial picture with mental note to revisit by rereading after completion of reading.

- Seeking to remember information primarily by matching it/linking it to her prior knowledge.

- Utilizing text layout and chunking information, grouping ideas in the 'blocks' they were presented in within the text.

- Actively looking for typographical clues to utilize to signal to her the most important information. She was searching for the hierarchical relationship between ideas in text as signaled by the typographical features. S13(PF) utilized diagrams and non-linguistic text features to check and clarify her understanding of Passage concepts.

- Noticing and being sensitive to coherence and stylistic features, noting features of style and matching them to previously encountered genre. S13(PF) demonstrated sensitivity while reading by noticing and evaluating the relative difficulty of the language and grammar difficulty in the text.

- Noticing when texts possessed many 'facts and figures' and being sensitive to the density of these. Greater density led to greater perceived text difficulty. Texts were judged for their perceived difficulty and the degree to which they dealt with 'facts or theory'.

- $\quad$ Preferring to learn through stories.

- Sometimes evaluating whether she agreed with the concepts being put forward by the reading.

- Guessing unknown words primarily from context and occasionally from recognized morphological elements like negative prefixes.

- Identifying and noting key terms but often minus the meaning they were embedded in. 
- Identifying main idea units throughout the text. She seeks the gist, seeking its match to what she already knows, and fills in many details from her own world knowledge, life (especially workplace) experiences and prior learning.

- Addressing comprehension breakdowns primarily by re-reading.

From the many forms of analysis it has been possible to piece together an understanding of S13(PF)'s task representation and modus operandi. For S13(PF), success in 'reading to understand and remember' means:

- using textual clues to identify main ideas, seeking the gist of the passage and retelling this using the words from the Passage together with a great deal of her own understanding and responses.

In comparing $\mathbf{S 1 3}(\mathbf{P F})$ 's results to previous similar studies it was interesting to find that Block (1989, p.477) records a reader who appears to share many of the characteristics of $\mathbf{S 1 3}(\mathbf{P F})$ 's habitual responses to text. Block describes a reader called "Janette", by saying that "she used information from her own life to fill in the many gaps in her understanding, her responses focused more on her own experiences than they did on the text".

Thus, S13(PF)'s approach is best likened to a story teller. The Story-teller is, as their title suggests, a seeker and a teller of stories, searching primarily for the 'human interest' in text, she accurately seeks out main ideas and then runs with them, responding to them on a number of levels and weaving into them her own interpretations and filling in the 'gaps' with her own understandings. 


\section{(9.2.10) Weaving Together the Strands of S13(PF)'s Profile}

\section{Recommended Academic Reading Development for S13(PF)}

Based on the characteristics revealed by S13(PF)'s profile the following measures are recommended to assist $\mathbf{S 1 3}(\mathbf{P F})$ to address areas for development in her academic reading:

- Observation of S13(PF)'s reading approach indicates that she already has good selective reading at the sentence level (i.e. in identification of main ideas and directing attention to these) but may benefit from development of more selective approaches to whole texts.

- $\mathbf{S 1 3}(\mathbf{P F})$ may benefit from being assisted to utilize her favoured strategies of subvocalisation and reading aloud in ways that do not slow her reading and task completion.

- S13(PF) would benefit from guided practice in achieving greater balance between the big picture and attention to detail and enhancing the completeness and accuracy of her recall. (This may be achieved through more appropriate strategy use).

- S13(PF) may benefit from assistance in developing an ability to manage negative affective responses to texts that contain higher percentages of K2, AWL and Off-List items, since these were found to be most associated with perceptions of Vocabulary Difficulty for S13(PF).

- S13(PF) had a generally good attitude to reading the set text but was not inclined to read further and had an underdeveloped awareness of the idea of intertextuality and authorship of texts. By guided exposure to wider ranges of literature in the field she may be able to develop these and this 
would work with her natural desire to connect ideas to create a stronger discipline understanding.

○ S13(PF) needs considerable development of her general English vocabulary as well as modeled and guided practice to develop a wider repertoire of strategies for dealing with unknown vocabulary. A wider repertoire would assist her to lessen her dependence on unhelpful or timeconsuming vocabulary guessing strategies.

○ More detailed investigation is needed to ascertain the main causes of S13(PF)'s slow Reading Rate. These need to be addressed and she then needs to be assisted to interact with conceptually dense texts and those with greater percentages of technical words in a more efficient manner to enable her to manage the reading loads of higher study. S13(PF) will need assistance in addressing her use of unhelpful rate regulation strategies.

○ S13(PF) would benefit from developing greater awareness of the features and reference sections of the set text and how these can be utilized for her benefit (such as a glossary section to help her with vocabulary).

\section{Chapter Summary}

It became very apparent in the construction of all of the Course A Readers' profiles that they displayed idiosyncratic approaches to the same academic reading task, and this can be seen in the two Readers presented in this chapter. This finding concurs strongly with findings of other similar research. Mann's (2000, p.311) close analysis of the reading behaviours and beliefs of four case study participants reading the same passage established that the students had all come to "certain deductions" about academic 
reading. Each student defined the same task in a different way, had different approaches to the task, and thus, experienced it in their own, unique, routinised ways. These approaches appeared to be directly related to how each student conceived of academic reading and how one should best accomplish that task. From this analysis Mann (2000) came to the conclusion that viewing academic reading as "a purely neutral, cognitive process" is insufficient, and that it must in fact be understood within its personal, socio-cultural and wider political context.

The importance of documenting these Readers' task conceptions is based on the need to "look beyond learners' actions and behaviours to understand the beliefs motivating these behaviours", because research has shown that what readers believe about the nature of knowledge, reading and learning influences their reading behaviour (Schraw \& Bruning,1996; Armstrong, 2008, p.211). In other words, in research into academic literacy it is important to not only know what students do but why they do it, and this project has sought to shed light on this.

This chapter has served two main purposes:

1) to illustrate how the holistic profiling of student readers can be achieved by weaving together all of the threads previously discussed, (i.e. to showcase the profiling method)

2) to profile the real Pacific individuals as Readers in the higher education context and highlight the uniqueness of each Reader (i.e. to showcase the Readers themselves). 
Holistic consideration of the strands of the Reader profiles has enabled recommendations to be made for the Pacific Readers' individual academic reading growth in terms of their cognitive, metacognitive, strategic, effectiveattitudinal, and linguistic development.

These two holistic profiles are indicative of the diversity that exists within the body of students referred to as "Pacific", and highlight the need to systematically build an understanding of the strengths and weaknesses of Readers in order to plan their academic support and development activities. In doing so, such programmes will better suit the actual needs of real readers, rather than adopting the general 'one size fits all' approach and persistently striving to place readers in pre-determined programmes of development, which may be perhaps more for our own convenience as educators than as a truly student-centred response to our readers.

The following and final chapter of this thesis pulls together the final threads of the work, addressing Research Questions 3 (c) and (e) relating to generic understandings of the Readers, the matches and mismatches that exist between them and their context, and the link between academic reading profile characteristics and academic success. 
CHAPTER 10

\section{CONCLUDING THOUGHTS}

This final chapter 'ties off' the strands of the project and views the patterns now revealed in the complete weaving. It addresses Research Question 3(c): What matches and mismatches exist between the academic reading profiles of the Pacific first year readers and the typical 'good reader' in this Faculty? And Research Question 3(e): Is there a link between academic reading profile characteristics and academic success?

The chapter is presented in the following sections:

(10.1) Matches and mismatches between the characteristics of the first year Pacific Reader and the 'Good Reader' in Course A

(10.2) Is there a link between academic reading profile characteristics and academic success?

(10.3) Concluding thoughts

\section{(10.1) Matches and Mismatches between the characteristics of the first year Pacific Readers and the 'Good Reader' in Course A}

My results thus far strongly suggest that there is no 'one right way' to read in higher education, but for argument's sake however, let us assume that there is a 'better' way (as opposed to a 'right' way) to be a reader in Course A. We will call it the way of the 'Good Reader', whose characteristic approach was identified in Chapter 6. Essentially in Course A students are not only required to decode, they are also required to understand the meaning, critically evaluate the message, remember the content, and apply the new-found knowledge flexibly and creatively. This section touches on the main matches and mismatches between the 'Good Reader' and the real Pacific Readers as 
revealed in this study and in this way provides an insight into the degree of 'fit' between the Pacific Readers and their new academic literacy context.

Table 10.1: Comparison of the characteristics of a 'good reader' in Course A \& observations of these characteristics in the Pacific student case study readers

\begin{tabular}{|c|c|}
\hline 'Good Reader' characteristics & $\begin{array}{l}\text { Whether these Pacific Readers } \\
\text { demonstrated these characteristics }\end{array}$ \\
\hline $\begin{array}{l}\text { Able to deal successfully and } \\
\text { independently with texts high in } \\
\text { academic, technical and } \\
\text { specialist vocabulary. }\end{array}$ & $\begin{array}{l}\text { The ability of these Readers to deal with } \\
\text { the vocabulary profiles of their texts } \\
\text { varied greatly. Vocabulary sizes were } \\
\text { found to be limited, as were strategies for } \\
\text { dealing with unknown vocabulary in text. }\end{array}$ \\
\hline $\begin{array}{l}\text { Able to deal successfully and } \\
\text { independently with } \\
\text { grammatically complex texts. }\end{array}$ & $\begin{array}{l}\text { Grammatical complexity had varying } \\
\text { effects on these Pacific Readers. On the } \\
\text { whole it did not seem to affect their } \\
\text { interest or task success, but did affect their } \\
\text { perceptions of difficulty, and thus may } \\
\text { have had indirect negative impacts on } \\
\text { Readers. }\end{array}$ \\
\hline $\begin{array}{l}\text { Able to deal successfully and } \\
\text { independently with } \\
\text { conceptually dense texts. }\end{array}$ & $\begin{array}{l}\text { The density of ideas in text may not have } \\
\text { impacted Readers' task success or interest } \\
\text { perceptions, but Readers were negatively } \\
\text { affectively impacted by the density of } \\
\text { ideas. }\end{array}$ \\
\hline $\begin{array}{l}\text { Able to deal successfully and } \\
\text { independently with texts } \\
\text { containing highly varying types } \\
\text { and amounts of non-linguistic } \\
\text { features. }\end{array}$ & $\begin{array}{l}\text { It was found that Readers responded } \\
\text { varyingly to non-linguistic material in text. } \\
\text { On the whole however it can be said that } \\
\text { layout does usually affect readers, and } \\
\text { non-linguistic features like diagrams etc } \\
\text { are utilized. Texts without these features } \\
\text { are usually perceived to be 'boring' or } \\
\text { difficult. }\end{array}$ \\
\hline $\begin{array}{l}\text { Awareness of author and } \\
\text { recognition of intended } \\
\text { audience. }\end{array}$ & $\begin{array}{l}\text { Author awareness was very limited } \\
\text { amongst these Pacific Readers, and thus, } \\
\text { thought was seldom if ever given to } \\
\text { intended audiences of text. }\end{array}$ \\
\hline $\begin{array}{l}\text { Read to be able to participate in } \\
\text { class discussion and engage in }\end{array}$ & $\begin{array}{l}\text { Classroom observations indicated this did } \\
\text { not happen for those who had not done the }\end{array}$ \\
\hline
\end{tabular}




\begin{tabular}{|c|c|}
\hline $\begin{array}{l}\text { meaningful discussion with the } \\
\text { lecturer/tutor }\end{array}$ & $\begin{array}{l}\text { set readings before the class (and at the } \\
\text { times observed, this was the majority of } \\
\text { the class). }\end{array}$ \\
\hline $\begin{array}{l}\text { Read to understand key } \\
\text { discipline concepts, grasp basic } \\
\text { concepts and theories }\end{array}$ & $\begin{array}{l}\text { All Readers appeared to have this as their } \\
\text { main orientation, but this was driven } \\
\text { almost entirely by assessment. }\end{array}$ \\
\hline $\begin{array}{l}\text { Constantly seeking to integrate } \\
\text { topics and look for the } \\
\text { links/connections between them }\end{array}$ & $\begin{array}{l}\text { Only a few of the Pacific Readers } \\
\text { habitually read with this orientation. } \\
\text { Some never did. Concepts of } \\
\text { intertextuality were reasonably limited } \\
\text { amongst all Readers. }\end{array}$ \\
\hline $\begin{array}{l}\text { Analysing the content of } \\
\text { readings and engaging with } \\
\text { them critically }\end{array}$ & $\begin{array}{l}\text { While some Readers demonstrated the } \\
\text { ability to do this activity very few actively } \\
\text { did this while reading in this Course. }\end{array}$ \\
\hline $\begin{array}{l}\text { Read to recognize and examine } \\
\text { instances of the application of } \\
\text { theory in real life and determine } \\
\text { its applicability in novel } \\
\text { contexts }\end{array}$ & $\begin{array}{l}\text { Most of the Readers appeared to have } \\
\text { grasped that this was one of the main } \\
\text { reading outcomes expected of them. } \\
\text { However they still tended to only do this } \\
\text { when assessment required them to. }\end{array}$ \\
\hline $\begin{array}{l}\text { Read to form a basic } \\
\text { understanding to build on in } \\
\text { lectures. }\end{array}$ & $\begin{array}{l}\text { It was clear that many of the Readers did } \\
\text { not read before lectures, and the majority } \\
\text { did not read before tutorials. }\end{array}$ \\
\hline $\begin{array}{l}\text { Learn/understand the broader } \\
\text { context in which theories are } \\
\text { relevant }\end{array}$ & $\begin{array}{l}\text { It did not appear that any of the Readers } \\
\text { approached their readings in this manner. }\end{array}$ \\
\hline $\begin{array}{l}\text { Read to acquire the terminology } \\
\text { of the field }\end{array}$ & $\begin{array}{l}\text { By and large the Pacific Readers did } \\
\text { actively attempt to acquire the 'speak' of } \\
\text { the discipline, however they varied in the } \\
\text { extent to which they utilized unknown } \\
\text { lexis in text as a learning opportunity and } \\
\text { the extent to which they utilized glossaries } \\
\text { etc. }\end{array}$ \\
\hline Read beyond the set texts & $\begin{array}{l}\text { None of the case study Readers did this, } \\
\text { despite a couple of them tending to } \\
\text { voluntarily read widely in other interest- } \\
\text { driven contexts. }\end{array}$ \\
\hline $\begin{array}{l}\text { Read with an orientation to } \\
\text { deep understanding and long } \\
\text { term remembering }\end{array}$ & $\begin{array}{l}\text { While some of the Readers may have done } \\
\text { so at times, in general this approach was } \\
\text { not characteristic of the Readers, with a } \\
\text { usual orientation towards assessment } \\
\text { related and short term oriented } \\
\text { understanding. }\end{array}$ \\
\hline
\end{tabular}


It appears therefore that there is some degree of match between the Pacific Readers and the prototypical 'Good Reader', which provides a strong base to work from, but there are also a number of key areas in which they differ. The greatest area of match was seen in the fact that all the Pacific case study Readers in Course A read to understand key discipline concepts, theories and key vocabulary, and most sought to apply them. The downside was however that this was clearly assessment-driven and thus, probably resulted in a shallower engagement with content than was desired of them.

The apparent areas of mismatch included the Readers' inability to deal successfully and independently with complex vocabulary, grammatical complexity, and conceptual density, and unhelpful/unattractive non-linguistic features. The Pacific Readers' current characteristics also suggested very limited author awareness, little engagement with text prior to lectures, thus hindering their ability to participate in class discussion and limiting their contributions to those generated from general knowledge or common sense. Findings also suggest varied integration of content within the Course readings, and very little evidence of intertextuality (none of the readers sought the broader context for theories or read beyond the set texts). Few read critically, and those that could, did not do so in this Course. Thus, any academic reading development initiative in Course A would need to incorporate, among other things, clear measures for the remedy of these specific issues.

There was not sufficient evidence found to point to these areas of match and mismatch as having directly stemmed from any clear overarching cultural basis within the group, however clear connections could be drawn between individual Readers' reported reading histories and certain traits seen in their current academic reading personae and their approaches to text in the tertiary context. Thus, indirectly, it may be that the influences of cultural, historical 
and societal literacy practices (or limitations in the range thereof) have been manifested in these Readers' current internal representations, beliefs and behaviours in higher education, ultimately playing a role in determining the degree of success experienced by the reader.

\section{(10.2) Is there a link between academic reading profile characteristics and academic success?}

From the Pacific Readers' profiles a shared collection of core meaning-making strategies and also a variety of approaches characteristic of each Reader's own 'modus operandi' were observed. When scrutinized in light of the association of what literature typically classifies as 'deep' or 'surface' orientations to text, these Pacific Readers' mechanisms reflect more of the characteristics usually associated with surface text interaction than they do with the processes thought to be indicative of a deep approach to meaning-making.

Having found this however, it is important to remember firstly that this characteristic is by no means unique to this group of readers, and secondly, that this in no way necessarily means that these Pacific Readers are not able to process text with a deep meaning-making orientation. Rather, it simply means that in this reading scenario, with these Texts and this particular set Task,

these Readers utilized a certain set of strategies and approaches with varying degrees of success. Whether they did so through conscious choice, because of their inability to process text any other way, or because of some feature of the research process or Free Recall protocol, is a matter for much further investigation.

However, given the centrality of the chosen Task, what we can draw from these results is an indication of the kinds of text interactions that these readers are likely to undertake in their first year of higher education. Having observed 
this it then remains to be decided what kinds of interactions would be profitable to encourage and whether there is room for development in certain areas of their response. It may be that there is need for a widening and growth of skill-sets, values, beliefs and motivations surrounding this and other core academic reading tasks in order to maximize their success as readers in higher education.

It was also observed that Readers possessed varying overall degrees of perceived interest and difficulty in the set texts in their course, and that this was in fact quite a good indicator of their general feelings about the course and at the end of the day, also of their level of academic success. The noncognitive factors (Alexander, Jetton, Kulikowich, 1995) within these Pacific Readers were a clear force, often determining how effective their learning from text was. The Readers were frequently subject to strong emotional responses to texts which influenced their self-efficacy and thus, ultimately had an impact upon their motivation to accomplish the set reading in the Course.

Of the six Course A case study Pacific Readers that form the focus of this study, only one is known to have achieved what the university would consider to be 'academic success' (i.e. a pass mark in the Course). Reader S4(M) withdrew from the Course, Reader S7(F) failed, Reader S12(M) also failed, while Reader S13(PF) initially failed but after applying for an exam remark, was able to achieve a pass grade (C) for this Course and was thus, the only known 'success' amongst the case study Readers. Reader S5(M)'s results are unknown, but it is known that he is still enrolled in tertiary study. Reader S9(F)'s results are not known, but it is possible that she may also have passed on this or subsequent attempts, as she is known to have since progressed on to further stages in her study within this discipline and regularly contributes to university events as a helper, student leader and volunteer. S4(M) continued 
his study but in another discipline, having withdrawn from Course A part way through the trimester. He is also heavily involved in 'extra-curricular' university activities, however has not had the level of academic success in his courses that could be expected given his reading strengths, and is now limited in the number of Courses in which he is able to enroll.

Student record access restrictions mean that the achievements and pathways of these Readers was not accessible to me and, for example, S7(F)'s continued enrolment is unknown. It is possible that her heavy sports involvement and probable selection to regional teams may have caused her to leave university to pursue her sporting career, perhaps to return to study later in life or at another institution. In S12(M)'s case it is likely that he would have continued at University but may have forsaken the Humanities disciplines that he struggled to make sense of in favour of the Sciences. S13(PF), despite several attempts at some courses, went on to complete her degree with her major in another discipline.

So is there a clear link between certain academic reading characteristics and academic success? In short, no. However what is outstanding in these results is the fact that the traditional indicators of reading prowess - vocabulary knowledge, speed, and certain types of strategy use - have, on their own, not proven to be an accurate indication of these Pacific Readers' eventual success in the Course. In fact, it was those whom such measures would typically have characterized as 'proficient' and given no further developmental attention to who appeared to have been the most 'at risk' of failure in the course. Clearly, there is more to reading than the common measures used to assess it, and there is also more to academic success than just reading. This remains an area in great need of direct and intensive investigation within the New Zealand context. 
Issues of equity and inclusiveness in the learning environment and the need to equip students with the knowledge and skills they will need at each level begins in the first year of study. This study makes its primary contributions in documenting the nature of the assumed academic reading skill-set that characterizes the first year of higher education, and providing a tool-set with which the skill-sets of other first year disciplines and successive years can be mapped. Knowing this then sheds light on the actual academic reading skills and epistemologies students are expected to use/develop/acquire in this first year of higher education. It also enables the mapping of the characteristics of the reader themselves, and allows a useful critique of the degree of match and mismatch between them to then be carried out. Documenting the nature of these 'basic discipline skills' as they pertain to academic reading, will directly improve the 'transparency' that is needed for proper planning of the learning experience. Ideally both students and lecturers could come to share a clearer, more explicit, stronger, and more congruous conceptualization of what it takes to read effectively for study within their discipline, with acknowledgement and fostering of the expression of students' own originality and unique creativity.

The negative educational statistics seen in compulsory schooling levels in New Zealand in relation to reading and literacy are also seen in the saddening statistics for tertiary attrition and non-completion, and these statistics were, unhappily, borne out again in the small group of case study student readers in this study. The question of what should be done about this, and what factors could be associated with success for these Pacific Readers is challenging, given the extremely complex nature of reading itself and the largely undocumented nature of its role in tertiary study success or failure, as well as the scarcity of information available on the Pacific students' reading literacy at the tertiary level in New Zealand. It is hoped that this study raises awareness of core issues and the high stakes of this problem in New Zealand universities, 
and prompts a closer and more thorough focus on reading, the 'invisible' side of academic literacy in higher education.

\section{(10.3) Concluding thoughts}

\section{(10.3.1) ... About the Role and Nature of Reading in Higher Education}

A goal of this project was to closely document the characteristics of academic reading in higher education in order to better inform our efforts to adequately prepare and support readers in this context. The existence of the distinct 'ways' of reading within the various disciplines revealed by document analysis, observation and interviews in the three main case study Courses points towards the understanding of universities as places comprising multiple cultural and social contexts, each with their own ideologies, values, power relationships and socially sanctioned practices of literacy. This is where Hirst et al.'s (2004) question about how minority groups (such as students of Pacific descent) can be empowered to 'contribute to its ongoing conversations' becomes particularly pertinent. In seeking to answer this, we must also reframe the question as Dickie (n.d.) did, and ask: should we view their performance in terms of technical skill or from the point of view of culture and ideology? I believe it is essential that we do both.

The study has revealed both the obvious areas of strength possessed by our Pacific Readers, and the fact that areas of mismatch exist between their reading selves and their current target environment, and that these mismatches were both skill-based and ideological in nature. It was also clear in some cases that such mismatches were very likely to be the tangible out-workings and consequences of earlier educational experiences. In such situations I would argue that attempting skills training would most probably be fruitless unless work was done to first understand the readers' backgrounds, and to 
draw to conscious attention the ideological, belief and value systems which drive the Readers' idiosyncratic responses to text and academic reading tasks in higher education. It is likewise just as important that educators personally and then collectively examine the unseen motivations and beliefs behind their own expectations of student readers and their own pedagogical practice.

Such careful collective consideration of the implicit values and belief systems and the internal representations of academic reading held within institutions of higher education is vital to both the theoretical debate on transition and preparedness and to the practice of learning and teaching diverse students. Its consideration will inform decisions about whether/how we safeguard and equip students from oral culture traditions as they transition into an environment that is essentially the literate world's purest form of expression.

The practical findings of this study also revealed that the nature of texts differs lexically, grammatically, and in conceptual density from discipline to discipline, course to course, highlighting the often considerable disparity in the first year academic reading experience across the university. Further clear variation was visible in the sources of academic texts presented to first year students as set reading. The disparity between the 'imagined readers' in the minds of the texts' wide range of authors and the characteristics of these real Pacific student readers clearly varies by discipline according to text choices in the Courses, and is something that needs to be considered in courses where there is a large discrepancy between the two. I assert that when, in a first year course, texts with intended audiences that are far removed in nature from the actual student readers are presented to the readers with no apparent scaffolding to address this discrepancy, the educator is guilty of continuing (wittingly or unwittingly) to perpetuate the linguistic injustices and inequalities inherent in 
an education system in which students of certain linguistic or ethnic backgrounds continue to consistently 'under-achieve'.

There is little doubt that a great many first year students' enrolment decisions are frequently guided by the preceding reputations of the "easy course" and the "hard course". In part, the perpetuating of traditions and hierarchies of prestige between various disciplines/faculties almost appears to be 'permission' for these disparities to exist. This view is often held with the view that learning to read appropriately in any discipline is a 'rite of passage' usually achieved in a 'sink or swim' manner. The disparities observed in the first year experience present a heavily-laden issue, with much that needs to be unpacked by educators in open and constructive pan-university discussion if we are at all serious about the tentative steps being taken by NZ universities in the direction of mapping learning pathways. Before this perhaps loftier goal is achieved, these findings certainly provide food for thought for educators in relation to course design and assessment.

A further result worthy of mention is the finding that academic reading was related almost exclusively to assessment in the minds of the Pacific readers, and that lecturers and course designers had often deliberately 'tied' reading to assessment as a strategy for inducing its occurrence. Thus the observation of the 'forcing' of reading, and the somewhat unsurprising consequence that many of the student readers only read when compelled to by assessment, seems to reveal a vicious cycle within higher education. It strikes me that this is a rather unhealthy phenomenon which, when viewed objectively, reveals a system quite removed from the purer aspirations of scholastic endeavour, and indicates the participation of all parties in a rather elaborate game. 
Central also to this study is the understanding of reading as a socio-cultural practice, and the observation of 'reading cultures' in the form of integrated patterns of beliefs, behaviours, practices and knowledge actively created and perpetuated by those involved in these contexts. Through Lecturer and Tutor interviews as well as document analysis and classroom observation, this study has revealed the existence of privileged ways of interacting with text that are particular to the communities and contexts within the University, and constitute what Koo and Soo Hoo (2007) refer to as "sanctioned performances". The core academic reading tasks (and associated competencies) required of first year readers in the case study courses were found to involve reading for the following outcomes: recall, linking, analysis and application/demonstration in written form, critical thinking, and development of original thought and these were found to be comparable with similar studies worldwide. These core tasks, when analysed, revealed much about the reading practices considered 'appropriate' within the context of higher education (Kirkpatrick \& Mulligan, 1996).

As well as common and discipline specific core tasks, there were specific conceptions within Courses of what it meant to "assess, valuate, judge or interpret" in each of the discipline areas studied (Carter-Wells, 1996, p.52). For the main case study course, Course A, it was found that the specific academic reading approach involved reading to understand and apply, while in Course B it was reading to understand and compare, and in Course $\mathrm{C}$ it was reading to understand and critique. These main approaches to text each encompass their own belief and value systems and understandings (often implicit) of what it means to read/think/study/learn like a scholar within that particular discipline. The differences found between the disciplines once again raise the issue of the disparity of the first year reading experience. I raise this not to suggest that first year students are not capable of 328 
accomplishing the more complex tasks of critiquing, but rather to argue that the more complex the tasks required of readers, the greater the potential need for scaffolding of the reading experience.

In this study I also observed that underneath the various explicitly stated or implied reading tasks lay three broad, unstated learning objectives. Essentially, reading in the first year of university appeared to be being undertaken for the following three-part outcome: (1) the development of content, (2) the development of skills, (3) the development of thinking in the discipline. I present these as explicit means by which educators may examine their practice and pedagogy, and by which applied linguists might analyse the discourse of university classrooms to shed more light on what it means to read in the first and subsequent years of higher education.

In response to these findings, the challenge to educators is to strive to more thoroughly understand these cultures of reading and how student readers may be apprenticed into these communities more effectively, and moreover, how to strategically, overtly and actively assist readers to know how to find themselves as readers within their field of study (Behrman, 2004). The most important constraint for educators of Pacific and other minority descent readers is how to assist this to happen without losing, destroying or de-valuing who they are, and to do this in a manner free of the assimilationist trappings that seek to reduce and re-make rather than to enrich and empower.

\section{(10.3.2) ... About How to Develop Pacific Readers}

In finding the most suitable paths for developing Pacific and other groups of academic readers I do not believe that it is necessary to re-invent the wheel, just to make sure the right wheel is chosen to fit the vehicle at hand and the 
terrain intended for travel. In the worldwide literature reviewed in the course of this study I have gleaned insights into the development of readers which I judge to be of relevance to the Pacific readers in this study's context, and to the ethos of this work. Using these, and what we now know about the Readers and the academic reading context of the first year of university in New Zealand I am confident that the core values behind the ideas expressed in this section can be extracted and applied within specific educational contexts and matched to the needs of the student body and the resources at hand to generate relevant, strategic, timely, appropriate, and research-led responses to the academic literacy needs of students.

The most common approaches to reading development in higher education usually take the form of reading interventions, and commonly involve some form of reading strategy training. Song (1998, p.42) explains that "unsuccessful learners can improve their learning by being trained to use effective strategies", and as the empirical research base grew it became apparent that readers could in many cases be trained to be more strategic readers (Cohen \& Horowitz, 2002). Song (2002, p.41) believes that reading pedagogy, especially for adult English foreign language students in academic settings, should include "explicit and direct strategy teaching" through "direct explanation, explicit teacher modeling, and extensive feedback" (2002, p.52). It is important to note though that more time may be needed to allow strategies to be transformed from declarative to procedural knowledge, and to then integrate and automatize them (Peña Calvo, 2004). Interestingly, Salataci and Akyel (2002) found that this type of strategy teaching could occur in the second/foreign language reader's first language and that the process was bidirectional and interactive and appeared to provide support for the 'interactivecompensatory' model of reading. However, Carrell, (1985) cautions that 
seeking to master strategy should never displace the goal of reading for meaning.

In addition to strategy training, attention has also been given to the direct teaching and development of the kinds of reading processes that involve the higher order thinking skills, such as critical thinking. Wilson, Devereux, Macken-Horarik and Trimmingham-Jack (2004) showed that many students were not prepared at first for engaging with academic texts and that critical reading was a challenge to them. In describing how the students began to develop, employ and demonstrate critical reading skills when given extensive scaffolding and instruction, they warn of the danger of students regressing and disengaging with critical reading if the scaffolding is withdrawn before these critical reading skills are sufficiently embedded. They conclude that scaffolding students' reading experiences around critical reading "needs to continue into at least second year, and perhaps beyond" (Wilson, et al., 2004, p.7). To these researchers, the keys to success were (1) long term development of critical literacy practices and (2) its integration across course structures. Observations of the Pacific readers and the courses in this study suggest that this would be an ideal approach for the Pacific readers in the university studied.

Thistlethwaite (1990, p.587) adds the valuable observation that a powerful way to generate critical thinking is to "prescribe activities for co-operative problem solving and meaning construction", with an emphasis on social interaction and its opportunities for the modeling of effective thinking strategies, and this may have particular benefits as an approach for Pacific Readers. 
A comprehensive instructional program in English second language reading comprehension, and any form of developmental reading intervention or instruction in higher education should also include work on schema availability and schema activation (Carrell and Eisterhold 1983), metacognitive training (e.g., inference-awareness, analogy, advance organizers, reading guides) (Zhicheng, 1992, cited in Koda, 2005, p.221; Lin \& Chen, 2006; Hermida, 2009, p.5), comprehension monitoring skills, decoding skills, and similar core reading processes. Carrell (1985, p.742) also argues for the value of directly teaching various aspects of text structure, and concludes that such training on discourse types should constitute a part of any comprehensive instructional program in reading comprehension. The variations observed amongst the Pacific readers' ability to identify and exploit text structure for meaning making suggests this is indeed a valuable component of academic literacy development.

Author awareness, intertextuality, and an internal motivation to participate in the exchange, critique and evaluation of ideas are also features of a 'good reader'. At their present stage, most of these Pacific Readers had not fully developed these features, and tended to respond to texts in isolation, seeing them mostly as repositories of content to be understood and 'regurgitated' on demand. This points strongly to the argument that the provision of opportunities for the active development of these Readers from this inexperienced starting point is as important a part of higher education as the acquisition of content in the first year.

It is important also to acknowledge that these Course A case study Readers were all at varying stages in their journeys of self-awareness as readers. Some were found to be quite self-aware, however it was clear that there were also those who had very little self-awareness, and still yet there were those who had 
formed ambiguous or erroneous impressions of themselves as readers, demonstrating again a lack of accurate self-concept. As Lonon-Blanton (1993) points out, these readers were very much in need of being socialized into their discipline's discourse community and being encouraged to develop a 'reader persona' within themselves. Along with this ambivalence and lack of awareness of both self and others as readers, came related characteristics such as the unwillingness on the part of all but two of the Readers to act as active knowledge constructors, and to take a certain amount of authority in the meaning-making and negotiating while reading. Accordingly, the development of readers' self-awareness and self concept as readers is yet another crucial aspect of holistic reader development, which must also incorporate efforts to address the associated affective, attitudinal and motivational factors related to self-concept and self-awareness.

Furthermore, considering the insights revealed into the Readers' vocabulary sizes, direct attention to vocabulary development "through planned, systematic instruction must supplement all learning activities" is crucial (White, 2004). Deciding on whether and how this would work in the tertiary context is, I believe, up to the individual practitioner. In the Pacific context, Marshall and Gilmour's (1993) suggested pedagogical approaches for addressing the gaps in subtechnical and other types of lexis including:

- vocabulary pre-teaching

- content-related pre-reading activities

- modeling and scaffolding strategies for determining the meaning of unknown words

- extensive reading.

Vocabulary guessing strategies also feature regularly in suggested modes of redress for vocabulary-related issues in reading. In Readers' individual profile 
data a feature found to be common to nearly all the Pacific Readers was the need to develop a wider repertoire of effective strategies for dealing with unknown vocabulary - such as Fraser's (1989, p.80) Word Analysis Procedure, the use of phonics, structural and morphological clues, and inferencing from context (Nuttall, 1982, p.65). However in doing so, Laufer's (1997, p.28) observations about the unreliability of such strategies for word guessing and Pinker's "learnability paradox" (which acknowledges that one learns words by meeting them in natural contexts, but in order to make sense of those very contexts you need to know the words) must be borne in mind (Pinker, 1989).

Another angle at academic literacy development involves the use of various forms of technology. Dreyer and Nel's (2003) technology enhanced environment was found to be particularly successful in allowing reading strategy instruction to be self-paced and accessed in the privacy of students' own homes. In partnership with the potential benefits of technology, Dreyer and Nel (2003, p.362) also conclude that researcher and lecturer input is central, and that as educators

we must actively seek to share practices with colleagues that will help our students identify the obstacles that restrict their possibilities in university and equip all of the unique learners who fill our classes with the knowledge and strategies to take action toward transforming that which limits them.

In terms of the delivery of literacy development, there are virtually as many possibilities as there are contexts. Unfortunately, as Simpson and Nist point out, many programs within institutions still rely on generic models that use "either standardized tests or commercial materials to dictate what students will be taught". What is perhaps even more concerning is that "many of these 
commercial materials teach students only one study system (e.g. SQ3R) or a limited number of strategies, as if there were one best way for all students to read and study across all content areas" (Nist \&Simpson, 2000, p.535).

In order to become successful independent readers, English second/foreign language and even native speaking students need to "be able to carry out a wide range of comprehension and interpretive tasks" and build "skills and strategic resources" known by reading researchers to facilitate successful reading. This is best achieved through "extended reading practice and carefully developed long-term intensive reading instruction" (Grabe, 2010) through whatever form appropriate, be it embedded, bolt-on, supplemental or preparatory. The necessity of providing a wide range of opportunities for reading skill development has been reinforced by the unique and idiosyncratic nature of each reader's reading personae. Thus, both this study and the literature currently available relating to academic literacy development through instruction/intervention stress that academic literacy development is not simply a case of 'one size fits all', and that it must be clearly responsive to learners.

In the literature surveyed there is evidence of increasing support for the need for content teachers to expand their role to being reading teacher as well (Brown, 2007; Green, 2006, p.284). Hermida (2009, p.10) argues that when reading is placed at the forefront of the course, and where class activities are orchestrated to promote the exercise of higher-order cognitive skills to construct meaning from academic texts, students are more likely to take a 'deep approach' to reading and learning. Lecturer and tutor involvement in this study has shown that in order for this to happen, considerable groundwork is necessary in terms of bringing tertiary teaching staff to explicit awareness of that which they do, believe, and expect of learners. 335 
Academic staff involved in this study possessed widely varying degrees of conscious awareness of their own beliefs about reading in their discipline. Furthermore, it became apparent that the Lecturers/Tutors held quite different (and individually varying) conceptions of the nature and purpose and intended outcomes of academic reading in their courses from what the students themselves held.

Thus, whatever the form, context and modes of delivery selected for the specific context of literacy development, in essence what makes readers able to successfully meet the challenges of academic reading is probably not any one particular characteristic, but rather their own unique utilization of what they currently posses to generate a problem-solving response (be it affective, cognitive, metacognitive and/or strategic) that is characteristically activated when text challenge is experienced. A key feature of these persistent, successful readers is that they possess (1) a wide repertoire of ways to respond to text, (2) a clear knowledge of self, and (3) responsiveness to the text and task at hand. Educators therefore should not seek the elusive 'silver bullet' to academic reading literacy problems in higher education, but provide for the transitioning reader a series of planned experiences that will build within them the ability to call on the particular number and combination of characteristics and responses that will be most appropriate for the particular Text and the given Task at hand. This will in turn be shaped by an understanding of both the narrow and wider socio-cultural contexts in which they are operating at the time.

Observations within this study suggest that current practice in teaching and learning and academic literacy support and development is unlikely to be fully cognizant of, or properly responsive to, the diversity that exists amongst readers. This thesis argues that the way to respond appropriately to this 336 
diversity is by ensuring that diversity is matched by the provision of an appropriate range of support and development with flexible and diverse delivery options. Highlighting areas of match and mismatch between reader characteristics and discipline expectations is one means for enabling a practical and strategic response to real readers' needs. This reflects what Caplan and Nelson (1973) refer to as a 'Situation-centered approach' (with a system change orientation) rather than a 'Person-centred approach' (which focuses on the search for deficiencies within the person), and involves seeking solutions and approaches that are change-oriented within our institutions.

Teemant, Bernhardt and Roderiguez-Munoz (1997) provide a practical perspective on the importance of integrating skill and content instruction, and raise several very useful principles for the support of second language readers within mainstream content courses which can be applied to a higher education context, all of which have been supported by observations in this study. The first principle is that reading skill and content development go hand in hand, another is that affective and attitudinal factors influence reading, and their final principle is that there is much to be gained by a collective and shared responsibility for students' academic success, be they second language speakers or not.

What all approaches ultimately require is an acknowledgement that students' transition difficulties are due to (1) profound changes in the learning environment (such as has been demonstrated in this study), and (2) unfamiliarity with the lecturer's/discipline's expectations (as has also been demonstrated in this study), and are not necessarily a reflection of their 'academic impoverishment' (Green, 2006). Thus, in highlighting areas for development within these Pacific Readers I do not seek to continue to dwell on what makes readers deficient, but what makes them who they are, what 337 
defines the context they wish to succeed in, and how better to facilitate the match between the two.

What is needed is a move away from the view of these Readers as being in need of 'over-hauling' and 'make-overs' of their reading personae, to an acknowledgement, acceptance and celebration of the successful ways in which these Readers do interact with texts, and the use of these as a platform upon which to build and enrich in any reading areas in which a mismatch between the reader's skill set and those of their desired target discourse community. It constitutes a move away from the "persistent search for disabling attributes" toward the "search for a more accurate and complete characterization of second language readers" and the "enabling, rather than disabling, attributes of non-mainstream populations" (Jimenez et al. 1995, p.68)" (Mokhtari \& Reichard, 2004, p.379).

Devine (1988), Koda (2005), Auerbach and Paxton (1997), and Sengupta (2002) all stress the value of also exploring and tracing the development of the students' own understandings and conceptual models of reading. This opens the door to exciting and potentially emancipatory new approaches to literacy development in which students become partners in working to shape their conceptions and practices to become more effective in their new environment, namely the tertiary environment. In effect these readers become 'actionresearchers' studying their own reading processes. Pani (2004), for example, explores 'mental modeling' as an instructional method wherein teachers and learners became co-participators in the learning process. Wilson (2003) also describes how social constructionist approaches to reading offer tools and principles for reading (and content) teachers to draw students into energetic participation in text events, and to move them from outsiders to active participants who engage in active dialogue with texts and their authors. 
Such an approach may in many ways challenge the traditional beliefs of teachers/lecturers in higher education, but it is nevertheless extremely important for positive progress in literacy-related matters in higher education. The adoption of a strengths-based appraisal, and an approach to academic literacy development in the first year of higher education which recognizes and responds to diversity, enables the re-framing of these Pacific Readers as "at promise", rather than "at-risk" (Tabachnick \& Bloch, 1995).

I believe also that acknowledging and documenting the challenge of academic texts in these Faculties, and seeing them in conjunction with the demands of the tasks required of the readers, compels us to move beyond blaming the reader for their 'deficiency'. It necessitates an acknowledgement of the 'unnaturalness' and the 'hidden-ness' of what we do. It encourages educators to work systematically to once more take an objective look at the texts, tasks and readers, to recognize the challenges of their educational contexts, and to find innovative ways to bridge this gap in a true (as opposed to tokenistic) spirit of 'inclusiveness' in higher education.

\section{(10.3.3) ... About the Research Design, Processes, and Future Directions}

The central framework this thesis offers for conceptualizing academic reading in higher education as the dynamic interplay of Text, Task and Reader characteristics within a socio-cultural context has afforded rich insights into the authentic texts, actual tasks and real readers. Through the framework, the investigation of the interaction between two of the factors (Reader and Text) has afforded valuable insights into the meaning making processes of these Pacific case study Readers. Investigation through this framework also revealed how student readers' strategic approaches to text were shaped by the 
characteristics of the Text itself when undertaking the set Task, and the framework holds the potential to shed much light on the nexus between Text, Task and Reader in future research. The wide scope of this thesis means that its contribution is broad rather than deep. Each area explored in this study warrants its own future in-depth investigations, and the intention of this thesis was to weave a foundation upon which this future research and future discussion may take place. Of particular interest would be further and more clearly 'participant-constructed' investigation of the beliefs of students and staff about the role and nature of academic reading within the various sociocultural contexts of higher education, as well as the ways in which these bi/multi-cultural individuals transition between the various reading literacy contexts within their lives.

The nature of these case study Pacific Readers' most favoured meaningmaking approaches reveals that at this level and for readers with this degree of reading expertise, the areas for development lie primarily in the top-down processes of reading (i.e. skill, strategy and thinking processes associated with reading). The readers in this study were all found to possess levels of reading fluency and language proficiency that place them in a range which requires special consideration as to how to enhance their reading as a thinking process. This is not to say however that at this level of proficiency it can be assumed that the mechanical aspects of reading do not need addressing. The degree of diversity found in every strand of this study is evidence of this, and there are likely to be numbers of first year students who do in fact require direct attention concerning the mechanics of reading.

The recent development of the national Literacy for Adults Assessment Tool (Te Arapiki Ako, n.d.) means that there is now a standardized tool for assisting post-compulsory education systems within NZ to diagnose the needs and 340 
abilities of adult student readers with limited fluency. However the value of the prototype reader profiling framework developed in his study lies in its ability to provide educators and researchers with an understanding of readers who have for the most part already developed relatively high levels of reading and language skill, but who still find themselves falling short in their new academic context. The larger framework of the study which includes the profiling of context (texts and tasks and the socio-cultural context of literacy) then enables us to draw out and identify both skill and ideological gaps between the person and their environment, and thus target future educational and research efforts.

The study is however, heavily shaped and coloured by the fact that I am a practitioner, an educator who has worked closely with Pasifika students for over ten years, and am a researcher with an 'agenda'. My practice has both informed me and been vital to the motivation, ethos and ethics with which the study has been undertaken, however this very passion and the pragmatism necessitated by it brings its own challenges. My commitment to the holistic and multi-faceted profiling of the text, task and reader elements within this context likewise presents limitations. A limitation of the present study for example, is that many of the profile measures themselves (for both texts and readers) remain necessarily rudimentary at present due to the scope of the project.

The study also faces the tensions that can exist when seeking to strongly uphold core non-Western cultural values whilst attempting to ensure 'rigour' from a Western research perspective. Certain methodological constraints which were encountered whilst upholding core values and processes may have served to prevent my being able to collect data on certain aspects of the student experience, and may thus, ironically, have worked to temper the 341 
holism to which I am essentially committed. For instance, in upholding the strong Pacific values of relationship and restricting data collection methods to those which did not violate the development of trust and rapport with these young adult Pacific participants (such as the use of digital recording devices for initial interviews with personal and potentially face-threatening content) has meant that verbatim data to support thematic analysis has not been available in this study. Furthermore, by strictly adhering to the commitment not to disrupt participants' study and life schedules, I have not been able to ensure that all measures were completed by all participants, thus, tables appear in the findings in which there is missing data. These are tensions which will need to be explored and resolved as Pacific research capacity and commitment to culturally appropriate methodology is built.

It is however, the combination of the process, the values, the synthesis of existing theory for a pragmatic outcome, and the richness of the reader profiling that together hold important potential for higher education. Essentially, this study represents a multidimensional assessment of fit, which aligns implicitly with the widespread tradition within the social sciences of seeking to examine aspects of both the person and their environment in order to better understand both positive and negative aspects of their interaction (Schneider, 2001). The study's design strives to simultaneously understand and assess the various aspects of the environment which exert varying degrees of influence on an individual, and in such research a certain trade-off is therefore necessary between the big picture and the minutiae.

A further limitation is obviously the relatively small scale of the study in terms of participant numbers and the limited number of disciplines represented in the finer analyses. It is however anticipated that the issues revealed by this exploratory and descriptive study will drive considerably more, larger scale 342 
research projects. Of particular interest for further research for example, is how the reader and text interaction trends appear when observed across larger groups, between different Task types, and between different Faculties.

The use of the Free Recall protocol has been particularly useful for the study's process-oriented investigation of reading, but I am aware that, as Sadoski, Goetz and Fritz (1993, p.293) point out, "comprehension and recall are not identical constructs" and, as evidenced by the differences between Free and Prompted Recall scores, in Free Recall the readers "can fail to recall previously comprehended material", and these Pacific Readers did so. Thus, observations of the actual reading recall ability of these Pacific Readers are likely, if anything, to have been under-represented by this measure, and there may be some value in investigating variations on the recall measure to better capture readers' success in the chosen academic reading task.

As indicated, it is anticipated that the study's framework will provide the basis for continuing research and hopefully, after refinement of each of the profile strands measures, steps to facilitate the usability of the profiling system and its results by readers and educators may be undertaken. An example would be the conversion of each of the individual measures' results onto a single rating scale so that easily interpretable charts and visual representations of readers' profiles may be created. In order to do so, considerable attention would also need to be given to creating a systematic means by which the crucial qualitative data from interviews could be coded and rated. Further investigation would also be needed into the ways in which the measures may be streamlined for greater practical ease of administration and marking if adopted for use on a large scale within institutions of higher education. 
At the heart of the study is the belief that first year student readers' outcomes and experiences are a result of the dynamic interactions between reader-texttask in any given socio-cultural context and reading situation, as illustrated in the central framework of the study. Having created for the readers a holistic profiling system capable of recognizing individual uniqueness, and identifying each reader's areas of strength and weakness, and having also utilized the text and task profiling systems to gauge the challenge of the higher education reading context, I seek now to further refine these tools, and to see conversations begin around an appropriate series of approaches for the holistic development of Pacific and other readers as they transition into tertiary education.

\section{(10.3.4) ... About an Alternative to the Deficit Understanding of Pacific Readers' Academic Reading Personae}

The final thought I wish to present is an alternative understanding to the perception of a person's academic reading as a fixed or static concept, which tends to result in the espousal of a deficit approach. If we are striving not to view groups of students from certain linguistic or educational backgrounds as being 'deficient' in some way in the Western educational context, how then can we view them?

To me it involves an effort to account for the lifelong, and continual development and shaping of a Pacific student's academic reading and general reading skills sets, habits, strategies and attitudes as they journey along their particular life and educational paths. In this view a reader's persona is not a static product or fixed state upon which judgments of their 'aptness', 'completeness' or 'suitability' for a certain academic path (or worse yet, from which judgments about their intellectual abilities or potential are made), but rather that each reader's persona is being continually created and revised. It 
comprises 'strands', that loosely represent the various skills sets, habits, strategies and attitudes that are currently woven into that reader's persona as a result of the cognitive, social, cultural and affective places and spaces in which they have encountered reading literacy prior to entry into higher education.

When a set of these features has been 'woven' or formed in a certain context, their entry to a new reading context will necessitate attention being given to and work being done upon the strands. Some strands can be carried forward into the new context; some strands are no longer of any use in the new context; and the selection, laying on and weaving in of those new strands must be purposefully and skillfully undertaken, ideally under the guidance of a master weaver. What is more, these new strands must be carefully selected and incorporated so as to bring about the appropriate skill-set for the new context. The role of the educator/mentor/learning adviser/academic reading teacher is thus to gain an understanding of the shape of the academic reading literacy skill, as well as the attitude and belief set that readers bring with them, and to assist the reader to decide which strands continue in their usefulness, which strands are at an end, and which new strands to add in their place for this new context, and how to achieve this. The crux of the understanding is simply the need to view readers of any age or background as 'works in progress'.

The implication of this continual incremental development is that there will be the need for regular insights into their reading persona, the changes it is undergoing, and its congruence with its desired context, such as could be holistically captured by the frameworks presented in this study. This view resists the labeling or pigeonholing of readers into pre-ordained bands for the convenience of the educator. It celebrates the uniqueness of the reader and acknowledges the deep connectedness of the mind, will, and emotions of the readers. It defends the ability of learners to pursue knowledge in ways 345 
meaningful to them and appropriate to their chosen context, and maintains that it is the right of every learner to experience the conditions needed for reaching their academic potential.

Thus, the many strands are woven, and the mat of knowledge is made. It is ready now to serve as the foundation upon which many conversations may take place and much future work may be undertaken. 


\section{APPENDICES}

Appendix 1 A summary overview of the data collection procedures and the participants and courses involved

Appendix 2 Text samples selected for use in the study

Appendix 3 Course A Readers' Free Recall Transcripts

Appendix 4 Reading Strategies, Habits and Attitudes Inventory (RSHAI) \& Results

Appendix 5 Lexical profiles of sample passages in Courses A, B and C.

Appendix 6 Vocabulary size test results

Appendix 7 IU recall patterning in Course A Passages A7-A11

Appendix 8 Map of frequency of IU recall patterns within sentences in Course A (Passages A7-A11)

Appendix 9 IU analysis of Course A text sample Passages A6-A11 


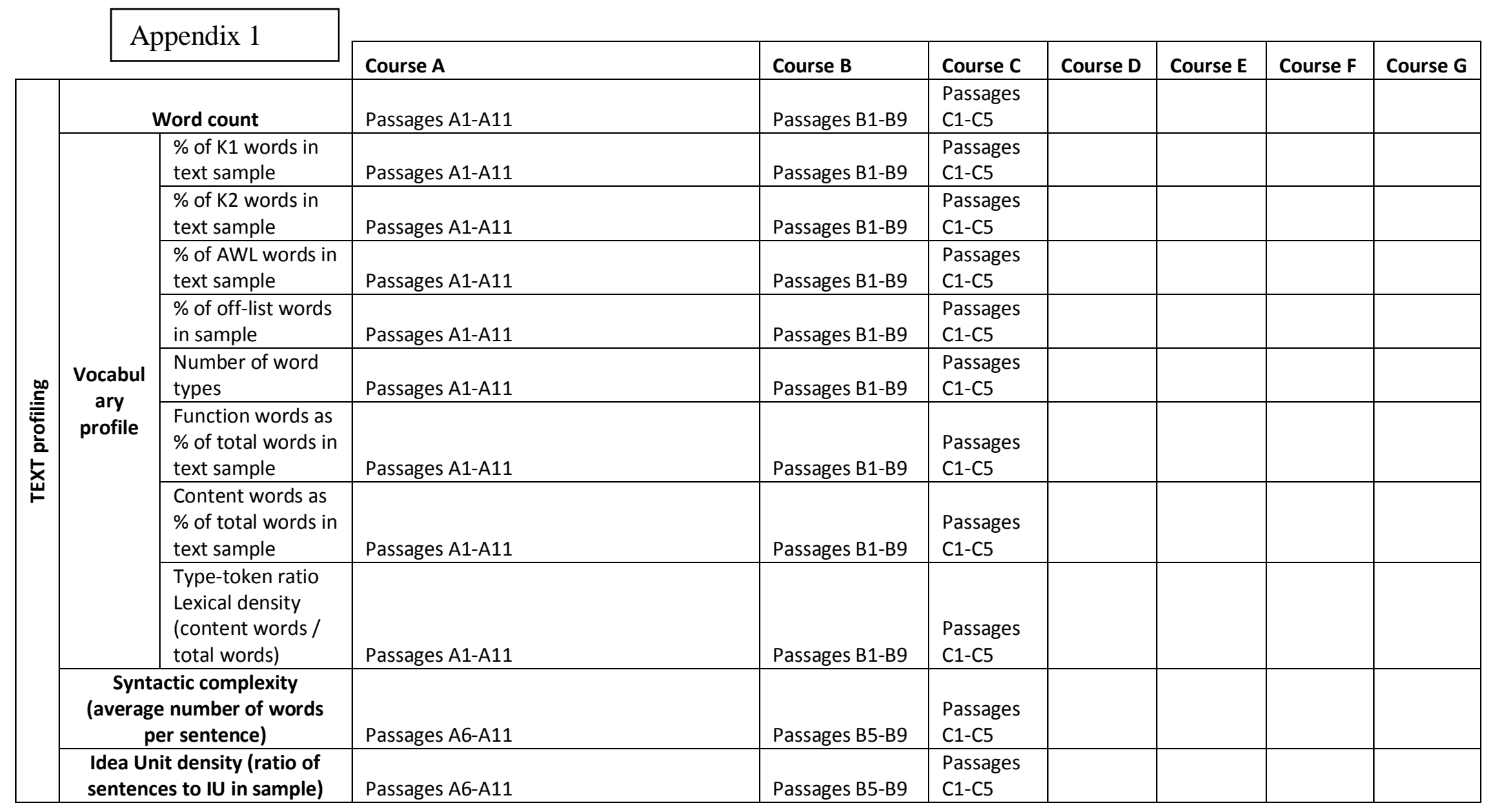




\begin{tabular}{|c|c|c|c|c|c|c|c|c|}
\hline & & Course A & Course B & Course C & Course D & Course E & Course F & Course G \\
\hline \multirow{3}{*}{ 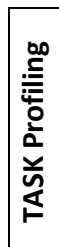 } & $\begin{array}{l}\text { Course Outline document } \\
\text { analysis and lecturer/tutor } \\
\text { participant checking }\end{array}$ & Yes & yes & yes & & & & \\
\hline & Lecturer/staff interviews & Yes & yes & yes & & & & \\
\hline & Classroom observation & Yes & yes & yes & & & & \\
\hline
\end{tabular}

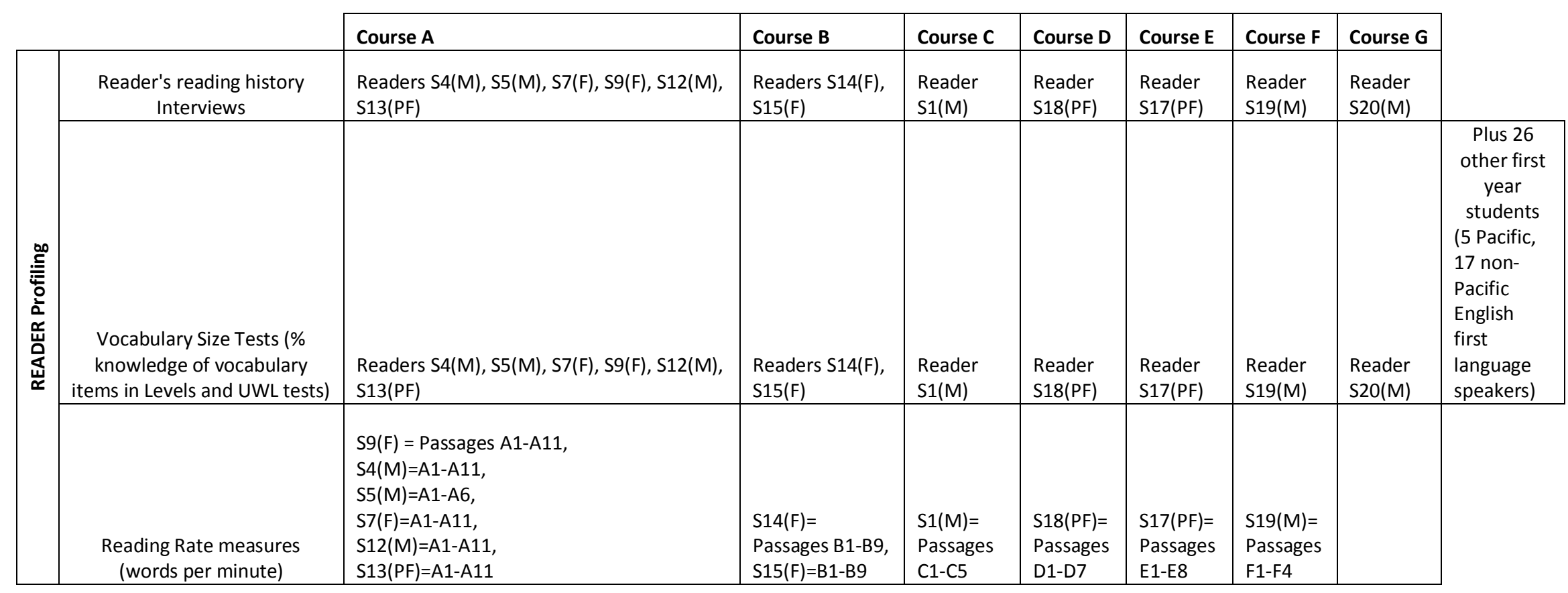




\begin{tabular}{|c|c|c|c|c|c|c|}
\hline $\begin{array}{l}\text { Free Recall Protocols (\% IU } \\
\text { recalled from passages) }\end{array}$ & $\begin{array}{l}\text { S9 }(F)=6 \text { Free Recall \& } 4 \text { Prompted Recall, } \\
\text { S4 }(M)=1 \text { Free Recall \& } 5 \text { Prompted Recall, } \\
\text { S5(M)= } 1 \text { Free Recall \& } 5 \text { Prompted Recall, } \\
\text { S7 }(F)=6 \text { Free Recall \& } 4 \text { Prompted Recall, } \\
\text { S12(M) }=6 \text { FreeRecall \& } 5 \text { Prompted Recall, } \\
\text { S13(PF) }=6 \text { Free Recall \& } 5 \text { Prompted Recall }\end{array}$ & & & & & \\
\hline $\begin{array}{c}\text { Academic Reading Strategies, } \\
\text { Habits and Attitudes } \\
\text { Inventory }\end{array}$ & S9(F), S4(M), S5(M), S7(F), S12(M), S13(PF) & S14(F), S15(F) & $\mathrm{S} 1(\mathrm{M})$ & $\mathrm{S} 18(\mathrm{PF})$ & S17(PF) & $\mathrm{S} 19(\mathrm{M})$ \\
\hline $\begin{array}{l}\text { Readers' perceived passage } \\
\text { interest ratings }\end{array}$ & $\begin{array}{l}\text { S9F)=Passages A6-A11 } \\
\text { S4(M)=Passages A6-A11 } \\
\text { S5(M)=Passage A6 } \\
\text { S7(F)=Passages A6-A11 } \\
\text { S12(M)=Passages A6-A11 } \\
\text { S13(PF)=Passages A6-A11 }\end{array}$ & & & & & \\
\hline $\begin{array}{l}\text { Readers' perceived passage } \\
\text { difficulty ratings }\end{array}$ & $\begin{array}{l}\text { S9F)=Passages A6-A11 } \\
\text { S4(M)=Passages A6-A11 } \\
\text { S5(M)=Passage A6 } \\
\text { S7(F)=Passages A6-A11 } \\
\text { S12(M)=Passages A6-A11 } \\
\text { S13(PF)=Passages A6-A11 }\end{array}$ & & & & & \\
\hline IU Recall mapping & $\begin{array}{l}\text { S4(M) (Passage A6 only), } \\
\text { S5(M) (Passage A6 only), } \\
\text { S12(M)(Passages A6-A11), } \\
\text { S13(PF)(Passages A6-A11) }\end{array}$ & & & & & \\
\hline $\begin{array}{c}\text { Free Recall task retrospective } \\
\text { interviews }\end{array}$ & S4(M), S9(F), S7(F), S12(M), S13(PF) & & & & & \\
\hline $\begin{array}{l}\text { Readers' final end of Course } \\
\text { interview }\end{array}$ & S4(M), S9(F), S7(F), S12(M), S13(PF) & & & & & \\
\hline
\end{tabular}




\section{Appendix 2: Text Samples Selected For Use in the Study Course A, B \& C.}

Course A

\begin{tabular}{|c|c|c|c|c|c|c|}
\hline 离 & Topic & Source & Page & $\begin{array}{l}\text { Word } \\
\text { Count }\end{array}$ & SC & IU Density \\
\hline A1 & Diverse workforce & $\begin{array}{l}\text { Jones, G. Management. The Aotearoa New Zealand } \\
\text { Context. John Wiley \& Sons Australia, Ltd: } \\
\text { Queensland, Australia. }\end{array}$ & 68 & 324 & & \\
\hline $\mathrm{A} 2$ & Tomorrow's Schools & $" \prime$ & 12 & 330 & & \\
\hline A5 & Sustainability & " & 82 & 493 & & \\
\hline A3 & Virtual Organisation & $\begin{array}{l}\text { Campling, J. Poole, D., Retha, W., Ang, S.E., Chan, } \\
\text { B., Tan, W_L., Schermerhorn, J.R. (2004). } \\
\text { Management. 3rd Asia-Pacific Edition. Queensland: } \\
\text { John Wiley \& Sons, Ltd. }\end{array}$ & $513-514$ & 391 & & \\
\hline A4 & Corporate Governance & $"$ & 233 & 349 & & \\
\hline A6 & $\begin{array}{l}\text { Communication } \quad \& \\
\text { Conflict }\end{array}$ & $"$ & 490 & 323 & 14.7 & 2.15 \\
\hline A7 & Career Development & $"$ & 344 & 297 & 20.85 & 2.28 \\
\hline A8 & Whistleblowers & $"$ & 155 & 340 & 21.62 & 2.68 \\
\hline A9 & Group input & $"$ & 454 & 329 & 19.8 & 2.05 \\
\hline $\begin{array}{l}\text { A } \\
10\end{array}$ & $\begin{array}{l}\text { Organisational } \\
\text { Performance }\end{array}$ & $"$ & 12 & 339 & 19.58 & 2.17 \\
\hline $\begin{array}{l}\text { A } \\
11\end{array}$ & Systems thinking & $"$ & 100 & 319 & 15.38 & 1.88 \\
\hline
\end{tabular}


Course B

\begin{tabular}{|c|c|c|c|c|c|c|}
\hline$\frac{\overline{8}}{\sqrt{9}}$ & Topic & Source & Page & $\begin{array}{l}\text { Word } \\
\text { Count }\end{array}$ & SC & IU Density \\
\hline B1 & Mantras & $\begin{array}{l}\text { Abe Ryūichi, "Word", in Donald, S. Lopez, ed., Critical } \\
\text { Terms for the Study of Buddhism (Chicago: University of } \\
\text { Chicago Press, 2005). }\end{array}$ & 306 & 332 & & \\
\hline B2 & Periodicity & $\begin{array}{l}\text { Mircea Eliade, The Myth of the Eternal Return (Or, Cosmos } \\
\text { and History) (Princeton: Princeton University Press, } 1991 \\
\text { [1954]). }\end{array}$ & $62-63$ & 276 & & \\
\hline B3 & Trance & $\begin{array}{l}\text { Felicitas D. Goodman, Where the Spirits Ride the Wind: } \\
\text { Trance Journeys and Other Ecstatic Experiences } \\
\text { (Bloomington: Indian University Press, 1990). }\end{array}$ & 15 & 261 & & \\
\hline B4 & Tangaroa & 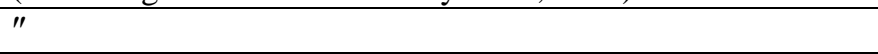 & 216 & 251 & & \\
\hline B5 & Battle of Karbala & $\begin{array}{l}\text { Kamran Scott Aghaie, The Martyrs of Karbala: Shi'i Symbols } \\
\text { and Rituals in Modern Iran (Seattle: University of Washington } \\
\text { Press, 2004). }\end{array}$ & $8-9$ & 320 & 29.05 & 2.57 \\
\hline B6 & Shi'i rituals & e & 12 & 200 & 19.9 & 2.3 \\
\hline B7 & The Past & $\begin{array}{l}\text { Joseph G. Jorgensen, "Religious Solutions and Native } \\
\text { American Struggles: Ghost Dance, Sun Dance and Beyond," } \\
\text { in Religion, Rebellion, Revolution: An Interdisciplinary and } \\
\text { Cross-Cultural Collection of Essays, ed. Bruce Lincoln (New } \\
\text { York: St Martin's Press, 1985). }\end{array}$ & 102 & 222 & 28.6 & 4.12 \\
\hline B8 & The Bear Dance & $"$ & 110 & 247 & 19.3 & 2.16 \\
\hline B9 & Mormonism & $"$ & 120 & 272 & 32.7 & 3.37 \\
\hline
\end{tabular}


Course C

\begin{tabular}{|c|c|c|c|c|c|c|}
\hline Label & Topic & Source & Page & $\begin{array}{l}\text { Word } \\
\text { Count }\end{array}$ & SC & IU Density \\
\hline $\mathrm{C} 1$ & $\begin{array}{l}\text { School \& Culture } \\
\text { Intro }\end{array}$ & $\begin{array}{l}\text { Hynds, A. (2008). Schooling and cultural reproduction. In J. } \\
\text { Codd, R. Harker \& R. Nash (Eds). Politicial Issues in New } \\
\text { Zealand Education }\left(2^{\text {nd }} \text { ed). Palmerston North: Dunmore }\right. \\
\text { Press. }\end{array}$ & 150 & 339 & 42 & 4.25 \\
\hline $\mathrm{C} 2$ & $\begin{array}{l}\text { School \& Culture } \\
\text { Discussion }\end{array}$ & $"$ & 160 & 238 & 33.5 & 3 \\
\hline $\mathrm{C} 3$ & $\begin{array}{l}\text { Childhood \& } \\
\text { Adolescence }\end{array}$ & $\begin{array}{l}\text { OECD. (nd.). Schooling for Tomorrow: OECD Scenarios. } \\
\text { Paris: OECD. }\end{array}$ & 2 & 503 & 22.6 & 2.45 \\
\hline $\mathrm{C} 4$ & Youth First Project & $\begin{array}{l}\text { Smith. L., Smith, G., Holer, M., Kempton, M., Ormond, A. } \\
\text { et al. (2002). Do you guys hate Aucklanders too? Youth: } \\
\text { Voicing difference from the rural heartland. Journal of } \\
\text { Rural Studies, Vol.18. }\end{array}$ & 170 & 291 & 32.2 & 3.44 \\
\hline $\mathrm{C} 5$ & Field Research & $"$ & 172 & 328 & 33.07 & 3.9 \\
\hline
\end{tabular}




\title{
Appendix 3: Course A Pacific Readers' Free Recall transcripts for Passages A6-A11
}

\author{
Reader S9(F) \\ Passage A6 \\ was not digitally recorded - my notes only
}

\section{$\underline{\text { Passage A7 }}$}

um, career development is talking about this British author, um, that he wrote his new book, um, think it was, the un-age of unreason I think, um, his name was Charles Handy, and he was just talking about how um, career development is very important now, um, talking about different ways how um, businesses can improve career development for individuals, um, explained in that passage was what career meant and it means um, a job where you have for um, a long period of time, um, usually people get good careers from, um, previous education and career pathway is when you've been at one career for a long period of time, and yeah, so it should be marked career development, and how, um, businesses can improve career development for individuals where they are satisfied and motivated within that business ... um, personal development, sequence, um, yeah

\section{$\underline{\text { Passage A8 }}$}

ah, [laughs], whistleblowers, something about, um, it sees the misdeeds of organizations, um, there was a case in it about, I think, California, I'm not sure, of um, sending questions about um, contaminating drinking water, and then, something about tobacco [laughs], um, and just, um, making it important to organizations where in other organizations they have had particular, um, staff members focus on ethical behaviours ... that's all I can remember ... Eric something...no [laughs]

\section{$\underline{\text { Passage A9 }}$}

um, it was just about um, making it easier for groups to work together as a team, um, how they apply the tasks within their business or management, um, businesses and management are just trying to make it easier for groups to work well as a team by um, providing more comfortable venues, kind of thing, with the example that's in that reading with St Luke's Advertising Agency in London, um, they focused on having a big room with computers and tables instead of profession, oh, ah, more formal venues for groups to work in, and um, like tables, tables where um, where they, where groups can work with others, yeah, more informal ... um, I think something about characteristics 
Passage A10

um, I think it was about how resources and customers are the two main important topics in the, um, open-systems organization, um, and something about productivity and performance efficiency I think, um, product, oh, if, performance efficiency is when in, for an example in a information system company kind of thing, um, they require, oh, they only get, um, they only get, what are they called? Um, they have like a time limit that they have to work at so, like, for example, if they're in a um, factory where they have to make a lot of keyboards but they have to make it under a time limit, yeah, that's all I can remember...um, fast food restaurants

\section{Passage A11}

um, it was about systems, oh, a system is um, little parts that are used to um, make up like, a function, I think, and a subsystem is smaller parts that form that larger system, um, they use an example of a guy I think in 19, I don't know his name but in 1938 he set up um, oh, he, he wrote a book, um, where he focused on what he had learnt because he was a manager of a organization I think on, oh, I think he was the executive for a telephone company or something, um, it was just talking about um, different systems that you can use as an organization, and there was this diagram too, talking about different systems where both input, oh, you can have input and output in it, there was like five circles I think, yeah, ... um, finance, organisational function, um, yeah.

\section{Reader S4(M)}

Passage A6

um, in the passage I just read there is a five types of main conflicts that can, um, come up in the workplace, and you have, conflict can be just disagreements, just general disagreements, there, um, there's substantive conflict which is like, disagreements between like, distribution of awards, policies, goals, just, um, sort of like, yeah, I think like primary secondary type tasks or whatever in the workplace, um, and then there's emotional conflict which is just sort of like more personal, like if you don't like someone, and they just, like, resent between, um, people working together, and then there's dysfunctional conflict which is where, um, there's not enough conflict going on so then there's not enough work being done, and then, and then there's functional conflict which is constructive, and um, that's when tasks are going quite well, and so, yeah, the dysfunctional conflict that's the conflict that's destructive so that's not very good, and um, the diagram showed that, like, the total, like the level of intensity going across the bottom and like low or high, like for the tasks the area they are being done in, and um, sort of um, the area you want to be in is sort of somewhere around the middle and that's where the inverted U showed at the top where um, the constructive conflict was quite high but the intensity wasn't too much or too less and it was kind of set at a good kind of level whereas the, yeah, that's like constructive, and the dysfunctional conflict was where the, it was too broad, like it wasn't sort of the intensity was either low or high, and it was kind of one sided so there was no kind of place in the middle so tasks were either being done to a good standard or to a very low standard, and wasn't very constructive at all ... um, oh, like, when there's dysfunctional conflict the tasks can be not done to a good standard, and like, when um, you have emotional conflict if it's managed well you can get quite good, good work out of people if you learn how to manage that and how they work better, like, if they're sensitive to certain things then you 
want to kind of adapt and put them maybe into another job, another task would be more suited if something's going on and if you've got, like, emotional conflict, maybe you put people who work better together in to prevent, you know, sort of resent between each other then tasks can be done to a high standard

\section{Reader S5(M)}

\section{Passage A6}

um, basically talking about the kind of conflicts that can happen in an organisation, in a workplace, um, it sort of defines the types of conflict there can be, um, ... it's also says that if managed well, if these conflicts are managed well then they could turn out to be um, successful, but if they're managed bad then it would end up being quite bad, and, they categorized the types of conflicts, um, I think, they've got, they are substantive conflicts, which are sort of work-related stuff, like job assignments and just conflicts that people have over work, sort of work duties, stuff like that, um, ah, I think there's emotional conflict that is when people get angry or don't like someone or, and it also includes um, personality, personality clashes as well, there's another one, but I can't remember it, um, yeah and there was the little diagram which showed an upside down U shape, showing that um, dysfunctional um, conflict can, oh, too less can be really bad or too much can be really bad, and, functional conflict was on the top and the middle ... having functional conflict also creates more motivation and performance from the team that's managed well and those things will be less if that conflict wasn't managed well, yeah, ... dislike, fear, um, I remember something, conflict, but I can't remember what it was, it was the other one, I'm sure there was like three, substantive and emotional and there's like one other, can't remember.

\section{Reader S7(F)}

\section{Passage A6}

(was not digitally recorded - my notes only)

conflict and communication, conflict management. Two types of conflict, substantive conflict and emotional conflict. Conflict can be harmful for performance of a workplace. The consequences of conflict are dysfunctional and constructive. There should be a balance between the two types of conflict.

\section{Passage A7}

(was not digitally recorded - my notes only)

It's about careers. Charles Handy wrote a book called the Age of Unreason. It is about how we have to change with the times, a career is what they do for a living. Good employers do 1 on 1 mentoring with employees. Career path is the different jobs you have throughout your life. Career paths are varied.

\section{Passage A8}


(was not digitally recorded - my notes only)

it's about whistleblowers. It has examples of famous whistleblowers like Erin Brokovich. These people risked their lives in order to ensure that other organisations are doing the right thing. It's about preventing workplace fraud. It's about how the government has protected whistleblowers. Workplaces have rules that make it harder to be whistleblowers. Some organisations have the job of ethics adviser in these situations.

\section{Passage A9}

um, it's about group input and um, oh, how um, when, oh, it was about like the nature of the task was like really important in the group, like, oh, the more complex the task, like, need more information --unclear-- but it was like, the less, oh, the, like, --unclear--- the task it would be less, oh, um, I can't remember what but there was something about how um, how an agency, an advertising agency in London, oh, Saint Nicks, how they um, how they did their layout of their office to try and like, get rid of the hierarchy, like, stuff, oh yeah, to try and make it more um, like, creative and, um, it was so like they put, like, a desk in the middle of the office as you walk in, and it catered for like, laptops and stuff --unclear--- there was like, meeting offices around there and like benches and stuff, yeah, that's to help, help be more creative I guess, with the group, um, .... No, that's all I got, sorry [laughs]... oh, there was more about how, um, in the different, oh, the group sizes, they like, where six or seven, oh, yeah, something like that, that can be like too much and cause too much, like, conflict, the more people, like the more problems, whereas it's like, oh, something like, I didn't get the odd number part but odd number's good because it's, like it gets rid of the like chaos sort of thing (?), um nah [laughs], and, um, like when you get just like more diversity and they get --unclear--, you need diverse groups, yeah, um, that's all I got.

\section{$\underline{\text { Passage A10 }}$}

um, [whispers to self], what was that about?... oh, yeah, it's about how um, organisations, oh, I forgot the whole line, the value creation, um that's, that's sort of like what ----, where organisations um, make profit pretty much like when their revenues are higher than their costs, and not for profit organisations um, like, help service, like, oh, [laughs] maybe I've forgotten a that bit...yeah, they help um, the providing(?) of services, um, that decrease, ...oh, and then there was the productivity, um, which is like the quantity and quality of, ah, something, of, like work, oh, output, sorry, there's a wiser use of resources and it can be like divided(?) individually or in groups or organisations, and um, oh, I forgot the other term, but yeah, there's another one, oh, um, ... oh, I think that's all I can remember

\section{Passage A11}

um, well, a system is, like, a large set of subsystems that, like, that um, sort of interrelate? Is that the word? I don't know if that's the word, but yeah, oh, like relate anyway [laughs] to sort of like function together, sort of, yeah, they have like, management systems, accounting systems, information systems, marketing systems, and there were some more I forgot, um, so that will, like, help, like if one of the subsystems(?) stuff up, um, yeah, it will affect the other subsystems within that system, um, then, there's an open system, but I'm not sure what that was [laughs], ...um, there was um, oh, in the diagram the like suppliers were the input to the system and the customers were the output, oh, the outflow, ... um, I don't really understand what that meant though, um, what else was it about? Oh, what was that guy's name? Chester Barnard, um, I think it was 1987 or something like that, he 
wrote a book about this whole systems thinking and they still use it in management today and that, like, yeah, and they had some of the quotes from the book, but --unclear-- forgot them, um, ... yeah, that's it

\section{Reader S12(M)}

\section{Passage A6}

um, conflict, um, and different types of conflict, there was, um, there was one type that's to do with policies and goals and actions, that's substantive conflict and the other one 's emotional, so it's just people getting angry at each other, and um, there's constructive conflict which helps an organisation and destructive which doesn't help, or dysfunctional, I can't remember what it's called, and, ah, they had a graph there, and you want to be in the middle in terms of intensity of an argument, you want to be in the middle, not at the bottom or the top, like low intensity or high intensity, and, and ah, yeah, it's an important part in management ... um, dysfunction, constructive, intensity

\section{Passage A7}

career development and it started off with um, a book called The Age of Unreason and he said that times are changing and we need to change with them and then it went on the describe um, that very good employers um, offer services for developing careers and for their employees and they provide career development and after that it described um, um, it just gave a definition of career, it's like a series of jobs held for a period of time or something like that, and you can do that within an organisation or outside of it, involving more organisations, and that's about it ... yeah, external career development that's outside involving more than one organisation

\section{$\underline{\text { Passage A8 }}$}

um, about whistle blowers, ah, it started off with a number of examples of famous whistleblowers, there's Erin Brokovich, some cop that was ratting out bad cops um, a couple of other examples and specifics, and then it talked about a few possible retaliations um, a whistleblower might get, so like, um, like being dismissed um, yeah taking benefits from them and then it talked about laws that protect whistleblowers from retaliation um, in Australia and New Zealand I think it specifically seems that there were laws in place but they were in some cases inadequate and government officials I think received um, extra protection but that should be extended to the private sector, and, then it goes to um, safeguarding an organisation which involves the person, director um, president, I can't remember what it was called, and then it talked about a moral circle, but I've forgotten what that means

\section{Passage A9}

it was about teams, um, ... teams [laughs] ... and they gave one example of an organisation that changes its structure to, to help teamwork and um, it was just like instead of having individual offices there was a big table and so yeah, they would sit round there and, for talking, and that gets rid of hierarchy and you know, allows different people to share ideas, um, um, ... and then it talked about the size of teams um, and um, a big team might be 
too hard cos it's hard to manage everyone and um, you want an odd number of people so you don't get ties when you vote, um, that's all I can remember really

(the recorder was full so we could not record the other two. For one I have taken brief notes, and the other wrote his own recall down.)

\section{Reader S13(PF)}

\section{Passage A6}

oh, it's about, sort of communications, all sorts of communication within the organisation, it's how people communicate, and how people um, communicate with each other, ... oh um, like I said it's sort of um, all the sorts of communication you know, emotional, and um, emotional one, there's functional one, ... mmm [laughs], ... I think it was more like communication skills and how communication worked through, in an organisation

\section{Passage A7}

ah, it's about career development, um, it's, it's a, I like that, that paragraph, it's more like, um, encouraging, ah, the personal development in your career instead of um, organisation's development, it's a good thing, and you know how the first paragraph says time has changed, so um, it's true because we don't change the time, and ah, the time changes us [laughs] so, you know, as time goes by, and you want to change your career, it's a good thing ... oh, I like when they said, um, often, that the author say that he support of that thing, um, in an organisation um, it's important to encourage ah, personal development, you know, career and personal development, than encourage the whole organisation, and then he says, um, that's why it's important to have um, mentors and leadership, um changes, because he more like you wanted to do better and better, in your career and you might want to change your career sometimes ...um, it's the Australian one, the Australian author who says, and that's the research that's done, yeah I think it's mentioned Australia system or Australian research ... it says you know, it's, nowadays it's been important to have encourage the, in an organisation, um, personal development you know, than, personal career, personal development, because it's really important to employers

\section{Passage A8}

oh, it's about the ah, it's about whistleblowers, whistleblowers?, and unethical behaviours in organisations, like the example they found of that case in America when that cigarette company, like, they knew, and also the contaminating water, drinking water, they knew that they doing something wrong but they, it, unethical, is it unethical?, that they knew it but didn't want to say it because they um, that in Australia is the one that um, who suggested that it's wrong so what that ex, ex-police man was saying that it takes him four years to investigate that and, and he was the one who says whistleblowers [laughs] he started it I think ... so it's about unethical, even that word ethical, it's kind of complicated sometimes for me, it's, to interpret it, but that's what it's all about ... um, no, what that text, at the end what they're saying is that what the solution it says is, ah, offering or appointing staff to deal with that, that specific problem, you know like, running seminars for staff that, um, ... yeah I think so 
Passage A9

um, it's about how group input in an organisation, it was saying that um, that organisation achievements it relies heavily on how the um, the group communicates with each other, and resources and things they use, um, to , you know, to communicate um, also, um, talking about the, the um, big organisation and you know, like mem, team member, it's hard to manage, whereas a small organisation it's easier to manage and also voting, in terms of voting because the odd numbers, like it says there, six or seven members ... well it says there it's not a good number when it comes to voting and um, input, because it's hard to manage that but um, smaller teams is more easier ... mmm, nah, the other thing is it says there that the facilities that the team use like laptops and ah, technology um, is really relevant to the group input

Passage A10

um, it's about, the, the team work, and how um, value in everyone in the organisation, like with the, your performance, or personal development in the organisation is really important to the product and customer satisfaction is another important thing in an organisation because you rely on your products to sell and the customer satisfaction will increase, um, also it says there that productivity, that um, really important because, ... mm, the quality and the quantity increases because um, like, um, -unclear- . . mmm, na, there was one, it says there that if you're working in a factory, ah, for example if you're working in a computer manufacturing, so, um, deadline is really important because um, that will, um, you know, the whole organisation relies on you of the deadline, because the product has to be delivered on certain days so, that's really important

\section{Passage A11}

it's about, um, system thinking and the subsystem, so um, subsystems it's sort of like, it's a network of, organisation, like, it include marketing, and customer and supplies, it's a network of, of people involve in the organisation, ... um, subsystem is a small, um, system, it's like a, not a big system, but it's a small one for um, the organisation, like thinking, and planning, ah, yeah, I think that's it I can remember, ... ah, the customers and the suppliers and then inside was technology, marketing, purchasing, [laughs], so it's a network of how organisation works 


\section{Appendix 4: Reading Strategies, Habits and Attitudes Inventory \& Results}

\begin{tabular}{|c|c|}
\hline label & Statement \\
\hline $1 \mathrm{~A}$ & I like books that challenge me and provide explanations which go beyond the lectures and seminars \\
\hline 1B & I react to what I read in a range of ways, both intellectual and emotional \\
\hline $1 \mathrm{C}$ & Doing my academic readings is satisfying and productive experience for me \\
\hline $1 \mathrm{D}$ & I feel good about myself as a reader, I think I am good at reading \\
\hline $1 \mathrm{~F}$ & I am motivated to read by interest \\
\hline $2 \mathrm{~A}$ & I decide what to read closely and what to ignore \\
\hline 2B & I skip the less important parts of texts \\
\hline $2 \mathrm{C}$ & When I read I concentrate on learning only those bits of information I need to pass the assignment \\
\hline $2 \mathrm{D}$ & Before I read a text I preview it to get an idea of content and organisation \\
\hline $2 \mathrm{E}$ & when I read I look for and recognize the text's structure \\
\hline $2 \mathrm{~F}$ & I read in such a way as to avoid breaking the flow of meaning \\
\hline $2 \mathrm{G}$ & As I read I try to find relationships between ideas in text \\
\hline 2I & I take notice of the facts and figures in what I read \\
\hline $2 \mathrm{~J}$ & I am able to identify main idea information in what I read \\
\hline $2 \mathrm{~K}$ & It's OK to me if I don't understand everything in what I'm reading \\
\hline $2 \mathrm{~L}$ & I think about whether the content of the text fits my purpose for reading \\
\hline $2 \mathrm{M}$ & Before I read I make sure I am clear about my purpose for reading \\
\hline $2 \mathrm{~N}$ & Before reading I set a timeframe for completing my reading \\
\hline $3 \mathrm{~A}$ & I try to get back on track when I lose concentration \\
\hline 3B & While reading I actively try to stay focused on what I am reading \\
\hline $3 \mathrm{C}$ & I continually check my understanding while I read \\
\hline
\end{tabular}




\begin{tabular}{|c|c|}
\hline $3 \mathrm{D}$ & I notice and deal with gaps in my understanding of the text while I am reading \\
\hline $3 \mathrm{E}$ & I take notes while reading \\
\hline $3 \mathrm{~F}$ & I underline or circle information in the text while I read \\
\hline $3 \mathrm{G}$ & I write summaries after reading \\
\hline $3 \mathrm{H}$ & I mark or write on the text itself while I am reading \\
\hline $3 \mathrm{I}$ & I ask myself questions that I try to answer as I read the text \\
\hline $3 \mathrm{~J}$ & when I read I try to elaborate on the ideas in the text \\
\hline $3 \mathrm{~K}$ & I infer or guess meaning while I am reading \\
\hline $3 \mathrm{~L}$ & I try to simplify the ideas I find in texts \\
\hline $3 \mathrm{M}$ & While reading I try to predict or guess what ideas will come next \\
\hline $3 \mathrm{~N}$ & While reading I try to put ideas into my own words \\
\hline 30 & While reading I try to visualise or picture the ideas in my head \\
\hline $3 \mathrm{P}$ & I discuss my reading with others \\
\hline 3Q & I use reference material (such as dictionaries or encyclopedias) to help me understand words or ideas in the text \\
\hline $4 \mathrm{~A}$ & I make use of tables, figures and pictures in text to aid my understanding \\
\hline 4B & I make use of typographical clues like bold type or italics \\
\hline $4 \mathrm{C}$ & I make use of the titles, heading and subheadings in text \\
\hline $5 \mathrm{~A}$ & I often find myself questioning what I read \\
\hline $5 \mathrm{~B}$ & I evaluate the quality and value of what I read \\
\hline $5 \mathrm{C}$ & I make judgments about how the text is argued \\
\hline $5 \mathrm{D}$ & I often spot assumptions which have not been well argued in text \\
\hline $5 \mathrm{E}$ & I am sensitive to inconsistencies in the text \\
\hline $5 \mathrm{~F}$ & I compare attitudes and ideas discussed within the texts I read \\
\hline $5 \mathrm{G}$ & I am able to distinguish different kinds of reasoning used in text \\
\hline $6 \mathrm{C}$ & I adjust my reading speed according to what I'm reading \\
\hline
\end{tabular}


\begin{tabular}{l|l} 
6D & I adjust my reading speed depending on how difficult that part of the text is \\
\hline $6 \mathrm{E}$ & Whis
\end{tabular}

\begin{tabular}{l|l|}
\hline $6 \mathrm{E}$ & While reading I pause to think about the ideas in the text \\
\hline $6 \mathrm{H}$ & I read in broad phrases (several words at a time) \\
\hline 6I & I recognise words quickly when I am reading \\
\hline 7A & Before I read I try to think about how the text topic relates to what I already know \\
\hline 7B & I try to relate the ideas in text to what I have already learnt in that course \\
\hline 7C & I relate ideas that I read to my everyday experiences and world knowledge \\
\hline 8A & I make connections between what one author and other authors are saying \\
\hline 8B & I note the author's main arguments \\
\hline 8C & I think about the authors of what I read; their style, beliefs, intentions, historical context, etc. \\
\hline 9A & I try to guess the meaning of unknown words or phrases \\
\hline 9C & While reading I try to notice and learn new words/language items \\
\hline 1E & I don't feel good about myself as a reader, I don't think I'm good at reading \\
\hline 1G & I tend to stick closely to the course requirements and not do much extra reading \\
\hline 1H & I am motivated to read by fear of failure \\
\hline 1I & when I read I do it because I have to (external motivation) \\
\hline 2H & I find it challenging to understand the logical connections between ideas in text \\
\hline 3R & I concentrate on memorising a lot of what I read \\
\hline 6A & I like to read text aloud when I am reading \\
\hline 6B & I 'say' the words silently to myself in my head while I read \\
\hline 6F & While reading I re-read parts of text \\
\hline 6G & I read word by word (one word at a time) \\
\hline 9B & I look up most of the unknown words I come across when I read \\
\hline 9D & While reading I translate words or ideas into my first or other language/s to help me understand \\
\hline 9E & I sometimes lose the overall meaning while I am figuring out the words \\
\hline & \\
\hline
\end{tabular}


Key

\begin{tabular}{|l|l|}
\hline Item Category & Code \\
\hline Affective/Attitudinal & 1 \\
\hline Selective/Directed & 2 \\
\hline Comprehension & 3 \\
\hline Typographics & 4 \\
\hline Evaluation & 5 \\
\hline Reading Rate & 6 \\
\hline Prior Knowledge & 7 \\
\hline Author Awareness and Intertextuality & 8 \\
\hline Vocabulary & 9 \\
\hline
\end{tabular}

\begin{tabular}{|c|c|c|c|c|c|c|c|c|c|c|c|c|c|}
\hline & & Particip & Int Reade & If-rati & gs on $7 \mathrm{p}$ & int scale & & & & & & & \\
\hline $\begin{array}{l}\text { Positively or } \\
\text { Negatively } \\
\text { Framed items }\end{array}$ & $\begin{array}{l}\overline{0} \\
\text { త్ }\end{array}$ & 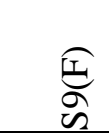 & 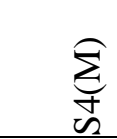 & $\sum_{\substack{n \\
n}}$ & 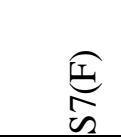 & 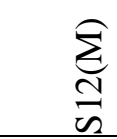 & 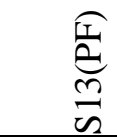 & $\frac{\overparen{I}}{\tilde{I}}$ & $\frac{\overparen{E}}{n}$ & $\underset{n}{\sum_{n}}$ & $\begin{array}{l}\mathbb{T}_{i} \\
\underset{\infty}{\infty}\end{array}$ & 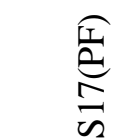 & 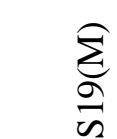 \\
\hline+ & $1 \mathrm{~A}$ & 4 & 3 & & 5 & 7 & 7 & 3 & 4 & 3 & 5 & 2 & 4 \\
\hline+ & 1B & 4 & 6 & & 3 & 4 & 5 & 5 & 4 & 7 & 5 & 3 & 2 \\
\hline+ & $1 \mathrm{C}$ & 7 & 5 & & 4 & 5 & 7 & 5 & 5 & 5 & 5 & 4 & 5 \\
\hline+ & $1 \mathrm{D}$ & 6 & 5 & & 4 & 3 & 5 & 3 & 5 & 5 & 5 & 1 & 5 \\
\hline+ & $1 \mathrm{~F}$ & 5 & 6 & & 6 & 7 & 7 & 7 & 7 & 7 & 7 & 6 & 5 \\
\hline+ & $2 \mathrm{~A}$ & 5 & 4 & & 6 & 3 & 1 & 3 & 6 & 6 & 5 & 6 & 5 \\
\hline+ & $2 \mathrm{~B}$ & 2 & 4 & & 6 & 5 & 2 & 4 & 4 & 5 & 6 & 5 & 4 \\
\hline
\end{tabular}




\begin{tabular}{|c|c|c|c|c|c|c|c|c|c|c|c|c|}
\hline+ & $2 \mathrm{C}$ & 6 & 4 & 6 & 3 & 4 & 5 & 6 & 6 & 6 & 4 & 6 \\
\hline+ & $2 \mathrm{D}$ & 5 & 4 & 6 & 2 & 5 & 3 & 2 & 6 & 5 & 5 & 5 \\
\hline+ & $2 \mathrm{E}$ & 6 & 4 & 6 & 3 & 5 & 4 & 5 & 7 & 5 & 4 & 4 \\
\hline+ & $2 \mathrm{~F}$ & 4 & 5 & 6 & 6 & 3 & 4 & 5 & 6 & 5 & 6 & 4 \\
\hline+ & $2 \mathrm{G}$ & 5 & 5 & 5 & 7 & 4 & 3 & 4 & 6 & 6 & 3 & 3 \\
\hline+ & $2 \mathrm{I}$ & 6 & 3 & 5 & 1 & 3 & 3 & 4 & 6 & 6 & 3 & 4 \\
\hline+ & $2 \mathrm{~J}$ & 6 & 6 & 6 & 6 & 5 & 5 & 5 & 5 & 6 & 4 & 5 \\
\hline+ & $2 \mathrm{~K}$ & 4 & 5 & 3 & 2 & 5 & 5 & 6 & 4 & 1 & 4 & 3 \\
\hline+ & $2 \mathrm{~L}$ & 6 & 3 & 6 & 5 & 2 & 3 & 4 & 5 & 7 & 4 & 5 \\
\hline+ & $2 \mathrm{M}$ & 4 & 4 & 2 & 2 & 7 & 3 & 4 & 5 & 6 & 5 & 6 \\
\hline+ & $2 \mathrm{~N}$ & 1 & 2 & 4 & 1 & 6 & 5 & 6 & 4 & 2 & 2 & 2 \\
\hline+ & $3 \mathrm{~A}$ & 5 & 4 & 4 & 5 & 3 & 5 & 6 & 7 & 7 & 4 & 6 \\
\hline+ & $3 \mathrm{~B}$ & 6 & 4 & 5 & 3 & 7 & 5 & 5 & 5 & 7 & 5 & 6 \\
\hline+ & $3 \mathrm{C}$ & 5 & 5 & 5 & 5 & 6 & 4 & 5 & 5 & 6 & 5 & 6 \\
\hline+ & $3 \mathrm{D}$ & 3 & 5 & 6 & 6 & 5 & 4 & 5 & 6 & 6 & 3 & 5 \\
\hline+ & $3 \mathrm{E}$ & 4 & 5 & 5 & & 6 & 6 & 4 & 4 & 5 & 4 & 5 \\
\hline+ & $3 \mathrm{~F}$ & 6 & 6 & 6 & 4 & 7 & 6 & 7 & 7 & 7 & 5 & 6 \\
\hline+ & $3 \mathrm{G}$ & 7 & 3 & 6 & 3 & 5 & 4 & 5 & 4 & 6 & 2 & 3 \\
\hline+ & $3 \mathrm{H}$ & 2 & 3 & 7 & 2 & 3 & 7 & 5 & 7 & 7 & 6 & 5 \\
\hline+ & $3 \mathrm{I}$ & 5 & 5 & 1 & 6 & 5 & 4 & 6 & 5 & 6 & 3 & 4 \\
\hline+ & $3 \mathrm{~J}$ & 4 & 3 & 4 & 2 & 7 & 5 & 6 & 5 & 4 & 3 & 5 \\
\hline+ & $3 \mathrm{~K}$ & 3 & 6 & 4 & 4 & 5 & 3 & 7 & 5 & 6 & 3 & 3 \\
\hline+ & $3 \mathrm{~L}$ & 5 & 4 & 6 & 3 & 5 & 5 & 5 & 5 & 5 & 3 & 4 \\
\hline+ & $3 \mathrm{M}$ & 3 & 4 & 2 & 5 & 5 & 5 & 6 & 4 & 6 & 5 & 2 \\
\hline+ & $3 \mathrm{~N}$ & 6 & 5 & 5 & 5 & 5 & 6 & 6 & 5 & 7 & 4 & 5 \\
\hline
\end{tabular}




\begin{tabular}{|c|c|c|c|c|c|c|c|c|c|c|c|c|}
\hline+ & 30 & 2 & 5 & 5 & 6 & 7 & 6 & 6 & 4 & 6 & 5 & 5 \\
\hline+ & $3 \mathrm{P}$ & 3 & 4 & 2 & 5 & 7 & 6 & 5 & 5 & 4 & 4 & 4 \\
\hline+ & $3 \mathrm{Q}$ & 2 & 5 & 5 & 6 & 5 & 6 & 2 & 6 & 5 & 2 & 5 \\
\hline+ & $4 \mathrm{~A}$ & 1 & 6 & 7 & 7 & 4 & 6 & 5 & 4 & 7 & 3 & 5 \\
\hline+ & $4 \mathrm{~B}$ & 5 & 4 & 7 & 7 & 4 & 5 & 5 & 5 & 7 & 6 & 4 \\
\hline+ & $4 \mathrm{C}$ & 7 & 4 & 7 & 6 & 5 & 6 & 6 & 6 & 7 & 6 & 5 \\
\hline+ & $5 \mathrm{~A}$ & 5 & 5 & 3 & 7 & 5 & 4 & 6 & 3 & 6 & 3 & 4 \\
\hline+ & $5 \mathrm{~B}$ & 3 & 5 & 4 & 5 & 4 & 3 & 5 & 5 & 6 & 3 & 3 \\
\hline+ & $5 \mathrm{C}$ & 5 & 4 & 5 & 5 & 3 & 3 & 5 & 5 & 6 & 3 & 4 \\
\hline+ & $5 \mathrm{D}$ & 3 & 5 & 5 & 6 & 4 & 4 & 3 & 4 & 5 & 3 & 3 \\
\hline+ & $5 \mathrm{E}$ & 2 & 5 & 3 & 6 & 4 & 3 & 3 & 4 & 6 & 3 & 5 \\
\hline+ & $5 \mathrm{~F}$ & 4 & 5 & 5 & 5 & 6 & 3 & 5 & 5 & 7 & 1 & 2 \\
\hline+ & $5 \mathrm{G}$ & 6 & 4 & 5 & 5 & 4 & 3 & 4 & 5 & 6 & 4 & 3 \\
\hline+ & $6 \mathrm{C}$ & 7 & 5 & 4 & 4 & 7 & 6 & 6 & 6 & 5 & 5 & 5 \\
\hline+ & $6 \mathrm{D}$ & 7 & 5 & 6 & 5 & 5 & 6 & 6 & 6 & 4 & 4 & 6 \\
\hline+ & $6 \mathrm{E}$ & 6 & 4 & 5 & 2 & 6 & 4 & 6 & 5 & 7 & 5 & 6 \\
\hline+ & $6 \mathrm{H}$ & 4 & 4 & 5 & 6 & 5 & 3 & 4 & 6 & 4 & 5 & 4 \\
\hline+ & $6 \mathrm{I}$ & 5 & 5 & 6 & 6 & 5 & 5 & 5 & 5 & 5 & 5 & 5 \\
\hline+ & $7 \mathrm{~A}$ & 4 & 5 & 4 & 5 & 5 & 5 & 6 & 6 & 6 & 5 & 4 \\
\hline+ & $7 \mathrm{~B}$ & 6 & 6 & 5 & 7 & 7 & 5 & 6 & 6 & 6 & 5 & 4 \\
\hline+ & $7 \mathrm{C}$ & 5 & 6 & 3 & 6 & 5 & 5 & 6 & 6 & 6 & 2 & 5 \\
\hline+ & $8 \mathrm{~A}$ & 4 & 4 & 5 & 6 & 5 & 2 & 4 & 5 & 6 & 3 & 3 \\
\hline+ & $8 \mathrm{~B}$ & 3 & 3 & 2 & 4 & 4 & 6 & 3 & 4 & 5 & 4 & 4 \\
\hline+ & $8 \mathrm{C}$ & 3 & 5 & 2 & 4 & 4 & 2 & 5 & 3 & 5 & 2 & 4 \\
\hline+ & $9 \mathrm{~A}$ & 5 & 4 & 5 & 5 & 5 & 5 & 6 & 6 & 5 & 6 & 5 \\
\hline
\end{tabular}




\begin{tabular}{c|l|l|l|l|l|l|l|l|l|l|l|l|l|}
+ & $9 \mathrm{C}$ & 4 & 6 & & 4 & 3 & 7 & 4 & 6 & 4 & 6 & 4 & 6 \\
\hline- & $1 \mathrm{E}$ & 2 & 2 & & 4 & 5 & 2 & 5 & 5 & 3 & 6 & 7 & 4 \\
\hline- & $1 \mathrm{G}$ & 6 & 4 & & 6 & 2 & 4 & 5 & 5 & 7 & 7 & 5 & 6 \\
\hline- & $1 \mathrm{H}$ & 7 & 4 & & 3 & 2 & 7 & 4 & 7 & 6 & 7 & 6 & 6 \\
\hline- & $1 \mathrm{I}$ & 6 & 4 & & 6 & 4 & & & & 5 & & 5 & \\
\hline- & $2 \mathrm{H}$ & 4 & 3 & & 3 & 2 & 4 & 5 & 5 & 6 & 6 & 4 & 4 \\
\hline- & $3 \mathrm{R}$ & 6 & 4 & & 7 & 3 & 5 & 5 & 6 & 5 & 6 & 2 & 6 \\
\hline- & $6 \mathrm{~A}$ & 5 & 4 & & 5 & 4 & 7 & 5 & 3 & 4 & 6 & 6 & 5 \\
\hline- & $6 \mathrm{~B}$ & 5 & & & 7 & 2 & 7 & 7 & 6 & 6 & 3 & & 7 \\
\hline- & $6 \mathrm{~F}$ & 7 & & & 7 & 2 & 7 & 7 & 7 & 6 & 5 & & 6 \\
\hline- & $6 \mathrm{G}$ & 3 & & & 6 & 2 & 7 & 5 & 6 & 7 & 4 & & 6 \\
\hline- & $9 \mathrm{~B}$ & 4 & 5 & & 3 & 6 & 7 & 5 & 1 & 5 & 4 & 3 & 5 \\
\hline- & $9 \mathrm{D}$ & 3 & 5 & & 1 & 1 & 7 & 3 & 5 & 2 & 7 & 4 & 4 \\
\hline- & $9 \mathrm{E}$ & 4 & 5 & & 3 & 5 & 5 & 2 & 3 & 4 & 5 & 6 & 3 \\
\hline
\end{tabular}


Appendix 5: Lexical profiles of sample passages in Courses A, B and C.

\begin{tabular}{|c|c|c|c|c|c|c|c|c|c|c|c|c|}
\hline & Course A & ext Samples & & & & & & & & & & \\
\hline & 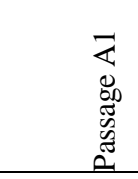 & 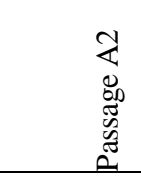 & 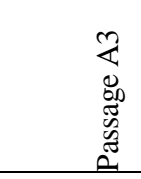 & 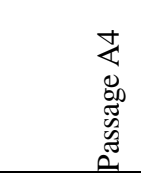 & 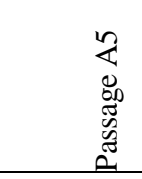 & 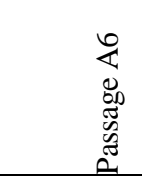 & 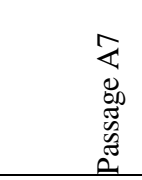 & 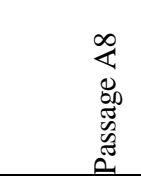 & 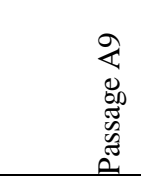 & 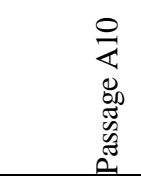 & 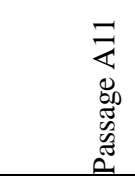 & 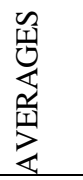 \\
\hline Word count & 324 & 330 & 391 & 349 & 493 & 323 & 297 & 340 & 329 & 339 & 319 & 348.5 \\
\hline $\begin{array}{l}\% \text { of } \mathrm{K} 1 \\
\text { words } \\
\text { in text sample }\end{array}$ & 76.06 & 74.77 & 70.51 & 76.07 & 63.37 & 57.66 & 73.67 & 66.38 & 71.56 & 80.24 & 72.5 & 71.16 \\
\hline $\begin{array}{l}\% \text { of } \mathrm{K} 2 \\
\text { words } \\
\text { in text sample }\end{array}$ & 6.97 & 6.91 & 7.24 & 6.27 & 5.54 & 8.11 & 4 & 9.48 & 6.42 & 7.96 & 6.25 & 6.81 \\
\hline $\begin{array}{l}\% \text { of AWL } \\
\text { words } \\
\text { in text sample }\end{array}$ & 9.39 & 9.31 & 13.94 & 10.54 & 11.68 & 23.72 & 12.67 & 9.48 & 18.04 & 10.32 & 13.75 & 12.98 \\
\hline $\begin{array}{l}\% \text { of Off-list } \\
\text { words } \\
\text { in sample }\end{array}$ & 7.58 & 9.01 & 8.31 & 7.12 & 19.41 & 7.51 & 8.67 & 14.66 & 3.98 & 1.47 & 7.5 & 8.65 \\
\hline $\begin{array}{l}\text { Number of } \\
\text { word types }\end{array}$ & 161 & 164 & 206 & 175 & 249 & 151 & 162 & 225 & 193 & 163 & 149 & 181.6 \\
\hline $\begin{array}{l}\text { Function } \\
\text { words as \% } \\
\text { of total words } \\
\text { in text sample }\end{array}$ & 43.03 & 45.65 & 40.48 & 42.45 & 34.65 & 34.23 & 45 & 35.06 & 40.67 & 41.3 & 39.38 & 40.17 \\
\hline $\begin{array}{l}\text { Content } \\
\text { words as \% } \\
\text { of total words }\end{array}$ & 33.03 & 29.13 & 30.03 & 33.62 & 28.71 & 23.42 & 28.67 & 31.32 & 30.89 & 38.94 & 33.13 & 30.99 \\
\hline
\end{tabular}




\begin{tabular}{|c|c|c|c|c|c|c|c|c|c|c|c|c|}
\hline in text sample & & & & & & & & & & & & \\
\hline $\begin{array}{l}\text { Type-token } \\
\text { ratio }\end{array}$ & 0.54 & 0.49 & 0.55 & 0.5 & 0.49 & 0.45 & 0.54 & 0.65 & 0.59 & 0.48 & 0.47 & 0.52 \\
\hline $\begin{array}{l}\text { Lexical } \\
\text { density } \\
\text { (content } \\
\text { words } \div \text { total } \\
\text { words) }\end{array}$ & 0.57 & 0.54 & 0.6 & 0.58 & 0.65 & 0.66 & 0.55 & 0.65 & 0.59 & 0.59 & 0.61 & 0.59 \\
\hline
\end{tabular}

\begin{tabular}{|c|c|c|c|c|c|c|c|c|c|c|}
\hline & \multicolumn{10}{|c|}{ Course B Text Samples } \\
\hline & 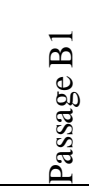 & $\begin{array}{l}\tilde{D} \\
\tilde{D} \\
\tilde{8} \\
\tilde{W} \\
\tilde{W} \\
\tilde{\sigma}\end{array}$ & 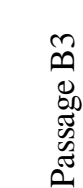 & 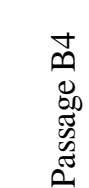 & 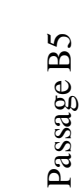 & 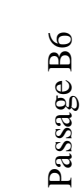 & $\begin{array}{l}\hat{\infty} \\
0 \\
\tilde{E} \\
\tilde{D} \\
\tilde{0} \\
\tilde{\sigma}\end{array}$ & $\begin{array}{l}\infty \\
\infty \\
0 \\
0 \\
\tilde{E} \\
0 \\
0 \\
0 \\
0\end{array}$ & 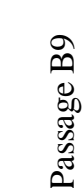 & 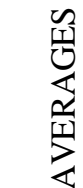 \\
\hline $\begin{array}{l}\text { Word } \\
\text { count }\end{array}$ & 332 & 276 & 261 & 251 & 320 & 200 & 222 & 247 & 272 & \\
\hline $\begin{array}{l}\text { \% of K1 } \\
\text { words in } \\
\text { text } \\
\text { sample }\end{array}$ & 66.98 & 81.75 & 73.28 & 80.16 & 73.19 & 68.78 & 71.49 & 67.09 & 75.18 & 73.1 \\
\hline $\begin{array}{l}\% \text { of } \mathbf{K 2} \\
\text { words in } \\
\text { text } \\
\text { sample }\end{array}$ & 4.05 & 2.55 & 6.11 & 3.97 & 2.42 & 2.93 & 3.4 & 16.03 & 5.47 & 5.21 \\
\hline $\begin{array}{l}\text { \% of } \\
\text { AWL } \\
\text { words in } \\
\text { text }\end{array}$ & 7.48 & 5.11 & 5.34 & 3.17 & 4.35 & 6.34 & 6.81 & 6.75 & 6.2 & 5.72 \\
\hline
\end{tabular}




\begin{tabular}{|l|l|l|l|l|l|l|l|l|l|l|}
\hline sample & & & & & & & & & & \\
\hline $\begin{array}{l}\% \text { of Off- } \\
\text { list words } \\
\text { in sample }\end{array}$ & 21.5 & 10.58 & 15.27 & 12.7 & 19.32 & 21.95 & 17.02 & 10.13 & 13.14 & 15.73 \\
\hline $\begin{array}{l}\text { Number } \\
\text { of word } \\
\text { types }\end{array}$ & 162 & 134 & 153 & 146 & 221 & 126 & 148 & 136 & 146 & 152.4 \\
\hline $\begin{array}{l}\text { Function } \\
\text { words as } \\
\% \text { of total } \\
\text { words in } \\
\text { text } \\
\text { sample }\end{array}$ & 44.24 & 52.92 & 47.71 & 46.03 & 44.2 & 42.93 & 44.26 & 38.82 & 48.18 & 45.4 \\
\hline $\begin{array}{l}\text { Content } \\
\text { words as } \\
\% \text { of total } \\
\text { words in } \\
\text { text } \\
\text { sample }\end{array}$ & 22.74 & 28.83 & 25.57 & 34.13 & 28.99 & 25.85 & 27.23 & 28.27 & 27.01 & 27.6 \\
\hline $\begin{array}{l}\text { Type- } \\
\text { token } \\
\text { ratio }\end{array}$ & 0.5 & 0.49 & 0.58 & 0.58 & 0.53 & 0.61 & 0.63 & 0.57 & 0.53 & 0.55 \\
\hline $\begin{array}{l}\text { Lexical } \\
\text { density } \\
\text { content } \\
\text { words } \\
\text { total } \\
\text { words) }\end{array}$ & 0.56 & 0.47 & 0.52 & 0.54 & 0.56 & 0.57 & 0.56 & 0.61 & 0.52 & 0.54 \\
\hline
\end{tabular}




\begin{tabular}{|c|c|c|c|c|c|c|}
\hline & \multicolumn{6}{|c|}{ Course C Text Samples } \\
\hline & $\begin{array}{l}\bar{U} \\
\tilde{E} \\
\tilde{\tilde{D}} \\
\tilde{\tilde{E}} \\
\tilde{\tilde{E}}\end{array}$ & 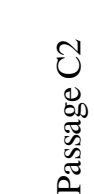 & $\begin{array}{l}\tilde{U} \\
\tilde{\Xi} \\
\mathbb{\Xi} \\
\tilde{W} \\
\tilde{D}\end{array}$ & 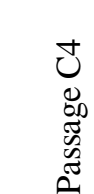 & 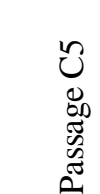 & 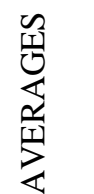 \\
\hline Word count & 345 & 340 & 476 & 291 & 328 & \\
\hline \% of K1 words in text sample & 67.54 & 69.12 & 80.67 & 72.51 & 71.79 & 72.32 \\
\hline$\%$ of $\mathbf{K 2}$ words in text sample & 5.22 & 7.35 & 5.88 & 6.53 & 5.36 & 6.06 \\
\hline$\%$ of AWL words in text sample & 8.12 & 11.76 & 8.61 & 12.71 & 16.07 & 11.45 \\
\hline$\%$ of Off-list words in sample & 18.55 & 10.88 & 4.83 & 8.25 & 6.79 & 9.86 \\
\hline Number of word types & 166 & 187 & 238 & 170 & 153 & 182.8 \\
\hline $\begin{array}{l}\text { Function words as \% of total words in } \\
\text { text sample }\end{array}$ & 35.07 & 37.35 & 44.96 & 42.61 & 42.86 & 40.57 \\
\hline $\begin{array}{l}\text { Content words as } \% \text { of total words in text } \\
\text { sample }\end{array}$ & 32.46 & 31.76 & 35.71 & 29.9 & 28.93 & 31.75 \\
\hline Type-token ratio & 0.48 & 0.55 & 0.5 & 0.58 & 0.55 & 0.53 \\
\hline $\begin{array}{l}\text { Lexical density (content words } \div \text { total } \\
\text { words) }\end{array}$ & 0.65 & 0.63 & 0.55 & 0.57 & 0.57 & 0.59 \\
\hline
\end{tabular}




\section{Appendix 6: Results of Vocabulary Size Test and Academic Vocabulary Test}

\begin{tabular}{|c|c|c|c|c|c|c|c|c|c|c|c|c|c|c|}
\hline \multirow[b]{2}{*}{ Readers } & \multicolumn{14}{|c|}{ Estimated Percentage Knowledge of the Vocabulary Levels (\%) } \\
\hline & $\mathrm{K} 1$ & $\mathrm{~K} 2$ & K3 & K4 & K5 & K6 & K7 & K8 & K9 & K10 & K11 & K12 & K13 & K14 \\
\hline $\mathrm{S} 1(\mathrm{M})$ & 90 & 100 & 100 & 90 & 100 & 60 & 70 & 90 & 70 & 70 & 50 & 60 & 30 & 30 \\
\hline S5(M) & 100 & 100 & 100 & 70 & 100 & 80 & 70 & 100 & 50 & 70 & 60 & 90 & 60 & 60 \\
\hline S7(F) & 100 & 100 & 100 & 80 & 100 & 70 & 70 & 80 & 60 & 70 & 80 & 90 & 40 & 50 \\
\hline S9(F) & 90 & 100 & 90 & 70 & 80 & 60 & 40 & 40 & 30 & 20 & 30 & 40 & 0 & 10 \\
\hline S12(M) & 90 & 100 & 100 & 100 & 100 & 90 & 100 & 100 & 60 & 100 & 70 & 70 & 50 & 50 \\
\hline S13(PF) & 90 & 90 & 80 & 70 & 90 & 70 & 50 & 60 & 20 & 30 & 70 & 50 & 30 & 30 \\
\hline $\mathrm{S} 14(\mathrm{~F})$ & 100 & 100 & 100 & 100 & 100 & 90 & 70 & 100 & 50 & 90 & 50 & 80 & 50 & 70 \\
\hline $\mathrm{S} 15(\mathrm{~F})$ & 100 & 100 & 100 & 90 & 100 & 90 & 70 & 80 & 60 & 80 & 50 & 70 & 20 & 20 \\
\hline $\mathrm{S} 17(\mathrm{PF})$ & 100 & 90 & 100 & 80 & 100 & 40 & 60 & 80 & 60 & 70 & 60 & 70 & 20 & 40 \\
\hline $\mathrm{S} 18(\mathrm{PF})$ & 90 & 90 & 60 & 60 & 90 & 60 & 50 & 60 & 40 & 70 & 50 & 50 & 30 & 30 \\
\hline S19(M) & 90 & 100 & 90 & 70 & 100 & 80 & 30 & 60 & 50 & 60 & 50 & 80 & 60 & 40 \\
\hline Other readers & $\mathrm{K} 1$ & $\mathrm{~K} 2$ & $\mathrm{~K} 3$ & K4 & K5 & K6 & K7 & K8 & K9 & K10 & K11 & K12 & $\mathrm{K} 13$ & K14 \\
\hline SA & 90 & 100 & 100 & 100 & 100 & 80 & 90 & 100 & 80 & 80 & 80 & 80 & 80 & 60 \\
\hline SB & 90 & 100 & 100 & 100 & 100 & 90 & 90 & 100 & 70 & 90 & 100 & 80 & 80 & 80 \\
\hline $\mathrm{SC}$ & 90 & 100 & 90 & 90 & 90 & 70 & 80 & 90 & 20 & 60 & 40 & 30 & 40 & 50 \\
\hline SD & 100 & 70 & 100 & 70 & 100 & 60 & 80 & 100 & 30 & 80 & 70 & 70 & 50 & 70 \\
\hline SE & 100 & 100 & 100 & 100 & 100 & 100 & 100 & 100 & 100 & 90 & 100 & 100 & 70 & 80 \\
\hline SF & 90 & 100 & 100 & 100 & 100 & 100 & 100 & 100 & 100 & 100 & 100 & 80 & 80 & 90 \\
\hline SG & 100 & 100 & 100 & 100 & 100 & 100 & 100 & 100 & 70 & 100 & 100 & 60 & 70 & 60 \\
\hline SH & 100 & 100 & 100 & 90 & 90 & 70 & 90 & 80 & 60 & 90 & 80 & 80 & 80 & 40 \\
\hline
\end{tabular}




\begin{tabular}{|c|c|c|c|c|c|c|c|c|c|c|c|c|c|c|}
\hline SI & 90 & 100 & 100 & 90 & 90 & 80 & 90 & 90 & 80 & 90 & 90 & 90 & 80 & 90 \\
\hline SJ & 100 & 90 & 100 & 90 & 90 & 80 & 60 & 100 & 70 & 90 & 80 & 70 & 30 & 30 \\
\hline SK & 100 & 100 & 100 & 90 & 100 & 100 & 80 & 80 & 50 & 70 & 50 & 90 & 50 & 40 \\
\hline SL & 100 & 100 & 100 & 100 & 100 & 100 & 100 & 100 & 70 & 90 & 90 & 80 & 60 & 50 \\
\hline SM & 100 & 100 & 90 & 90 & 100 & 90 & 80 & 100 & 70 & 90 & 60 & 80 & 60 & 50 \\
\hline SN & 100 & 90 & 100 & 100 & 90 & 90 & 100 & 100 & 90 & 100 & 100 & 90 & 80 & 70 \\
\hline SO & 100 & 100 & 100 & 90 & 100 & 90 & 90 & 100 & 90 & 100 & 90 & & & \\
\hline $\mathrm{SP}$ & 100 & 100 & 100 & 100 & 100 & 90 & 90 & 100 & 80 & 90 & 100 & 90 & 80 & 60 \\
\hline SQ & 100 & 100 & 100 & 80 & 90 & 80 & 60 & 100 & 70 & 80 & 50 & 70 & 30 & 40 \\
\hline Sf (Pacific) & 100 & 100 & 90 & 80 & 90 & 70 & 80 & 100 & 40 & 100 & 80 & 90 & 70 & 60 \\
\hline $\mathrm{Sg}$ (Pacific) & 90 & 100 & 90 & 80 & 80 & 70 & 50 & 80 & 50 & 80 & 80 & 40 & 40 & 20 \\
\hline Sh (Pacific) & 100 & 90 & 100 & 90 & 100 & 90 & 70 & 90 & 50 & 90 & 70 & 70 & 40 & 30 \\
\hline Si (Pacific) & 100 & 100 & 100 & 80 & 80 & 60 & 80 & 50 & 40 & 80 & 50 & 70 & 30 & 40 \\
\hline $\mathrm{Sj}$ (Pacific) & 100 & 90 & 60 & 70 & 80 & 40 & 30 & 70 & 60 & 50 & 40 & 20 & 40 & 30 \\
\hline $\mathrm{Sa}$ & 100 & 80 & 90 & 70 & 80 & 30 & 70 & 90 & 50 & 50 & 70 & 20 & 50 & 30 \\
\hline $\mathrm{Sb}$ & 100 & 80 & 70 & 70 & 60 & 50 & 50 & 50 & 30 & 20 & 50 & 50 & 30 & 40 \\
\hline $\mathrm{Sc}$ & 80 & 70 & 70 & 60 & 80 & 60 & 40 & 50 & 30 & 50 & 30 & 30 & 30 & 30 \\
\hline $\mathrm{Sd}$ & 90 & 90 & 100 & 90 & 90 & 60 & 60 & 90 & 50 & 60 & 70 & 60 & 50 & 50 \\
\hline $\mathrm{Se}$ & 100 & 90 & 80 & 70 & 80 & 50 & 70 & 60 & 40 & 50 & 30 & 70 & 50 & 30 \\
\hline
\end{tabular}




\begin{tabular}{|l|l|}
\hline & $\begin{array}{l}\text { Estimated \% } \\
\text { Knowledge of } \\
\text { the University } \\
\text { Word List word } \\
\text { families }\end{array}$ \\
\hline S1(M) & $100 \%$ \\
\hline S4(M) & $100 \%$ \\
\hline S5(M) & $100 \%$ \\
\hline S7(F) & $100 \%$ \\
\hline S9(F) & $21 \%$ \\
\hline S12(M) & $100 \%$ \\
\hline S13(PF) & $77 \%$ \\
\hline S14(F) & $97 \%$ \\
\hline S15(F) & $97 \%$ \\
\hline S17(PF) & $100 \%$ \\
\hline S18(PF) & $83 \%$ \\
\hline S19(M) & $80 \%$ \\
\hline & \\
\hline
\end{tabular}




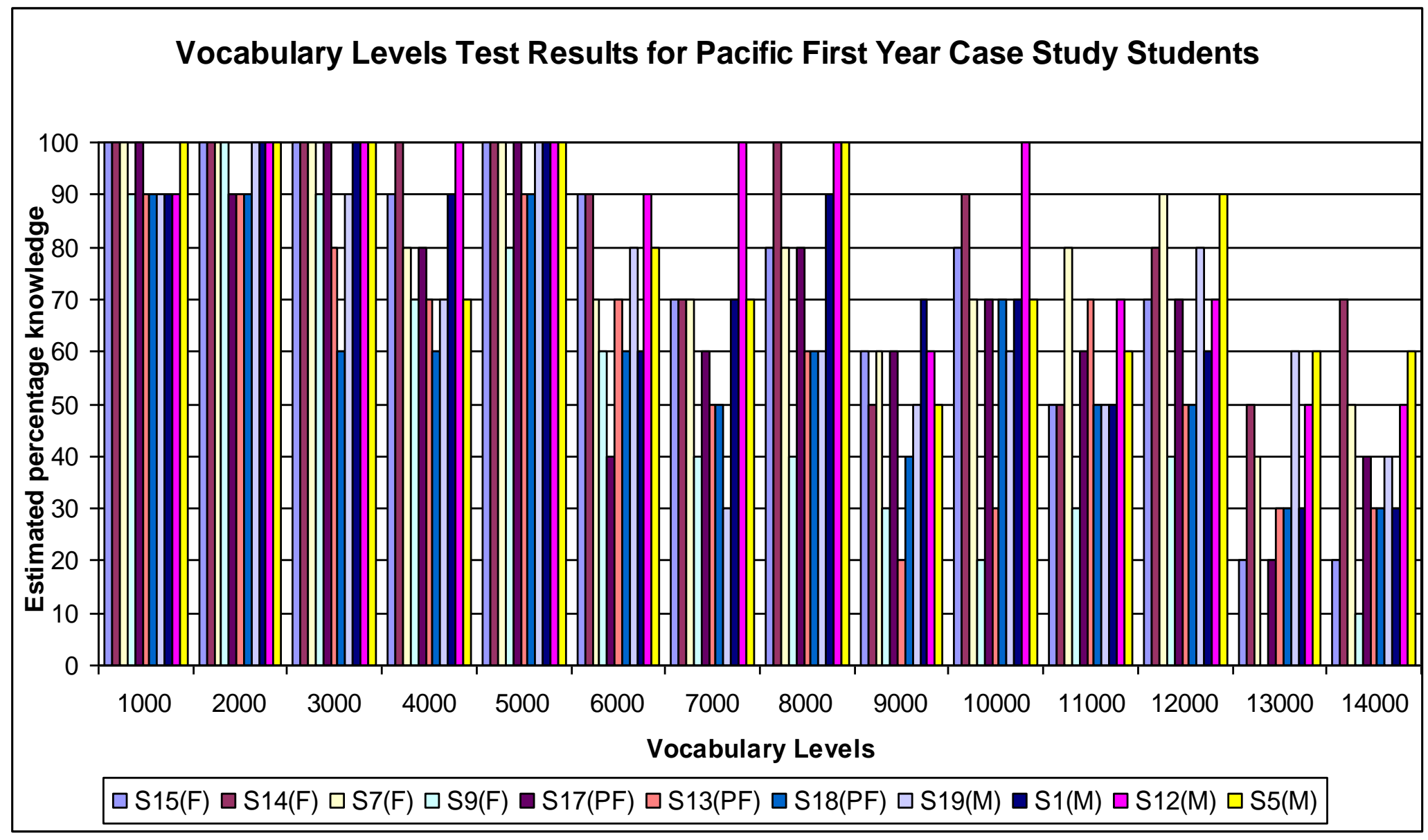




\section{Appendix 7: IU recall patterning in Course A Passages A7-A11}

Passage A7

\begin{tabular}{|c|c|c|c|c|c|}
\hline \multirow[t]{2}{*}{$\begin{array}{l}\text { IU Label } \\
\#\end{array}$} & \multirow{2}{*}{$\begin{array}{l}\text { Sentence IU is } \\
\text { found in } \\
\#\end{array}$} & \multicolumn{4}{|c|}{ Existence of IU in Student Free Recall } \\
\hline & & S7 & S9 & $\mathrm{S} 12$ & $\mathrm{~S} 13$ \\
\hline 1 & s1 & & $\checkmark$ & & $\checkmark$ \\
\hline 2 & s1 & $\checkmark$ & $\checkmark$ & & \\
\hline 3 & s1 & $\checkmark$ & $\checkmark$ & $\checkmark$ & \\
\hline 4 & s1 & & & & \\
\hline 5 & s2 & & & $\checkmark$ & $\checkmark$ \\
\hline 6 & s2 & $\checkmark$ & & $\checkmark$ & \\
\hline 7 & s3 & & & & \\
\hline 8 & s3 & $\checkmark$ & & & $\checkmark$ \\
\hline 9 & s4 & & $\checkmark$ & $\checkmark$ & $\checkmark$ \\
\hline 10 & s5 & & & $\checkmark$ & $\checkmark$ \\
\hline 11 & s5 & & $\checkmark$ & & $\checkmark$ \\
\hline 12 & s5 & & $\checkmark$ & & $\checkmark$ \\
\hline 13 & s6 & $\checkmark$ & & & $\checkmark$ \\
\hline 14 & s6 & & & & $\checkmark$ \\
\hline 15 & s7 & & & & \\
\hline 16 & s7 & & & & \\
\hline 17 & s7 & & & & \\
\hline 18 & s8 & & $\checkmark$ & $\checkmark$ & \\
\hline 19 & s8 & & $\checkmark$ & $\checkmark$ & \\
\hline 20 & s9 & & $\checkmark$ & & \\
\hline 21 & s10 & & & & \\
\hline 22 & s10 & & & & \\
\hline 23 & s10 & & $\checkmark$ & & \\
\hline 24 & s10 & & & & \\
\hline 25 & s10 & & & & \\
\hline 26 & s11 & $\checkmark$ & $\checkmark$ & $\checkmark$ & \\
\hline 27 & s11 & & $\checkmark$ & $\checkmark$ & \\
\hline 28 & s12 & $\checkmark$ & & & \\
\hline 29 & s12 & & & $\checkmark$ & \\
\hline 30 & s13 & & & & \\
\hline 31 & s13 & & & & \\
\hline 32 & s14 & & & & \\
\hline
\end{tabular}


Passage A8

\begin{tabular}{|c|c|c|c|c|c|}
\hline \multirow[t]{2}{*}{$\begin{array}{l}\text { IU Label } \\
\#\end{array}$} & \multirow{2}{*}{$\begin{array}{l}\text { Sentence IU is } \\
\text { found in } \\
\# \\
\end{array}$} & \multicolumn{4}{|c|}{ Existence of IU in Student Free Recall } \\
\hline & & S7 & S9 & $\mathrm{S} 12$ & $\mathrm{~S} 13$ \\
\hline 1 & s1 & $\checkmark$ & $\checkmark$ & $\checkmark$ & \\
\hline 2 & s1 & & $\checkmark$ & & $\checkmark$ \\
\hline 3 & $\mathrm{~s} 2$ & & $\checkmark$ & $\checkmark$ & $\checkmark$ \\
\hline 4 & s3 & & & & $\checkmark$ \\
\hline 5 & s3 & & & & $\checkmark$ \\
\hline 6 & s3 & & & & \\
\hline 7 & s4 & & & $\checkmark$ & $\checkmark$ \\
\hline 8 & s4 & & & $\checkmark$ & \\
\hline 9 & s4 & $\checkmark$ & & & \\
\hline 10 & s5 & $\checkmark$ & & $\checkmark$ & $\checkmark$ \\
\hline 11 & s5 & & $\checkmark$ & & \\
\hline 12 & s5 & $\checkmark$ & & & \\
\hline 13 & s5 & & & & \\
\hline 14 & s5 & & & & \\
\hline 15 & s5 & & & & \\
\hline 16 & s6 & & & & \\
\hline 17 & s6 & & & & \\
\hline 18 & s6 & & & & \\
\hline 19 & s7 & & & & \\
\hline 20 & s7 & & & $\checkmark$ & \\
\hline 21 & s7 & & & & \\
\hline 22 & s7 & & & & \\
\hline 23 & s8 & & & $\checkmark$ & \\
\hline 24 & s9 & & & & \\
\hline 25 & s9 & & & & \\
\hline 26 & s10 & & & & \\
\hline 27 & $\mathrm{~s} 10$ & & & & \\
\hline 28 & s11 & & & & $\checkmark$ \\
\hline 29 & $\mathrm{~s} 12$ & & & $\checkmark$ & \\
\hline 30 & s12 & & & & \\
\hline 31 & s12 & & & & \\
\hline 32 & s12 & & & & \\
\hline 33 & s12 & & & & \\
\hline 34 & s12 & & & & \\
\hline 35 & $\mathrm{~s} 12$ & & & & \\
\hline 36 & s13 & & $\checkmark$ & $\checkmark$ & $\checkmark$ \\
\hline 37 & s13 & & $\checkmark$ & & \\
\hline 38 & s14 & & & & \\
\hline 39 & s14 & & & & \\
\hline 40 & s15 & & & & \\
\hline 41 & s15 & & & & \\
\hline 42 & s15 & & & & \\
\hline 43 & s16 & & & & \\
\hline
\end{tabular}


Passage A9

\begin{tabular}{|c|c|c|c|c|c|}
\hline \multirow[t]{2}{*}{$\begin{array}{l}\text { IU Label } \\
\#\end{array}$} & \multirow{2}{*}{$\begin{array}{l}\text { Sentence IU is } \\
\text { found in } \\
\#\end{array}$} & \multicolumn{4}{|c|}{ Existence of IU in Student Free Recall } \\
\hline & & S7 & S9 & $\mathrm{S} 12$ & $\mathrm{~S} 13$ \\
\hline 1 & s1 & $\checkmark$ & & & \\
\hline 2 & s1 & & & $\checkmark$ & \\
\hline 3 & s2 & & & & \\
\hline 4 & s3 & & $\checkmark$ & & \\
\hline 5 & s4 & $\checkmark$ & & & \\
\hline 6 & s4 & & & & \\
\hline 7 & s5 & & & & $\checkmark$ \\
\hline 8 & $\mathrm{~s} 5$ & & $\checkmark$ & & \\
\hline 9 & s6 & & & & \\
\hline 10 & s6 & & & & $\checkmark$ \\
\hline 11 & s7 & & $\checkmark$ & $\checkmark$ & \\
\hline 12 & s8 & $\checkmark$ & $\checkmark$ & $\checkmark$ & \\
\hline 13 & s8 & $\checkmark$ & $\checkmark$ & $\checkmark$ & \\
\hline 14 & s9 & $\checkmark$ & $\checkmark$ & & $\checkmark$ \\
\hline 15 & s9 & & & & \\
\hline 16 & s9 & & $\checkmark$ & $\checkmark$ & \\
\hline 17 & s10 & $\checkmark$ & & & \\
\hline 18 & s10 & $\checkmark$ & & & \\
\hline 19 & s10 & $\checkmark$ & $\checkmark$ & $\checkmark$ & \\
\hline 20 & s10 & & & & \\
\hline 21 & s10 & $\checkmark$ & & & \\
\hline 22 & s10 & & & & \\
\hline 23 & s11 & $\checkmark$ & & $\checkmark$ & \\
\hline 24 & s11 & & & & \\
\hline 25 & s11 & & & & \\
\hline 26 & s12 & & & & \\
\hline 27 & s12 & & & & \\
\hline 28 & s13 & $\checkmark$ & $\checkmark$ & $\checkmark$ & \\
\hline 29 & s14 & $\checkmark$ & $\checkmark$ & $\checkmark$ & \\
\hline 30 & s15 & & $\checkmark$ & & \\
\hline 31 & s16 & & & & \\
\hline 32 & s16 & & & & \\
\hline 33 & s17 & $\checkmark$ & & & \\
\hline 34 & s17 & & & & \\
\hline 35 & s17 & & & & \\
\hline
\end{tabular}


Passage A10

\begin{tabular}{|c|c|c|c|c|c|}
\hline \multirow[t]{2}{*}{ IU Label } & \multirow{2}{*}{$\begin{array}{l}\text { Sentence IU is } \\
\text { found in } \\
\#\end{array}$} & \multicolumn{4}{|c|}{ Existence of IU in Student Free Recall } \\
\hline & & S7 & S9 & S12 & S13 \\
\hline 1 & s1 & $\checkmark$ & $\checkmark$ & & \\
\hline 2 & s2 & & & & \\
\hline 3 & $\mathrm{~s} 2$ & & & $\checkmark$ & \\
\hline 4 & s3 & $\checkmark$ & & & \\
\hline 5 & s4 & $\checkmark$ & & $\checkmark$ & \\
\hline 6 & s4 & $\checkmark$ & $\checkmark$ & $\checkmark$ & \\
\hline 7 & s4 & & $\checkmark$ & & \\
\hline 8 & s5 & $\checkmark$ & & & $\checkmark$ \\
\hline 9 & s5 & $\checkmark$ & & & \\
\hline 10 & s5 & & & & \\
\hline 11 & s6 & & & $\checkmark$ & \\
\hline 12 & s6 & & & & \\
\hline 13 & s6 & & & & \\
\hline 14 & s7 & & & $\checkmark$ & \\
\hline 15 & s8 & & & & \\
\hline 16 & s8 & & & & \\
\hline 17 & s9 & & & $\checkmark$ & \\
\hline 18 & s9 & & & & \\
\hline 19 & s9 & & & $\checkmark$ & \\
\hline 20 & s9 & & & & \\
\hline 21 & s10 & $\checkmark$ & $\checkmark$ & & \\
\hline 22 & s10 & $\checkmark$ & & $\checkmark$ & $\checkmark$ \\
\hline 23 & s10 & $\checkmark$ & & & \\
\hline 24 & s11 & $\checkmark$ & & & \\
\hline 25 & s11 & $\checkmark$ & & & \\
\hline 26 & s11 & $\checkmark$ & & & \\
\hline 27 & s12 & & & & \\
\hline 28 & s12 & & & & \\
\hline 29 & s12 & & $\checkmark$ & & \\
\hline 30 & s13 & & $\checkmark$ & & \\
\hline 31 & s14 & & $\checkmark$ & & $\checkmark$ \\
\hline 32 & s14 & & $\checkmark$ & & \\
\hline 33 & s15 & & $\checkmark$ & & $\checkmark$ \\
\hline 34 & s15 & & $\checkmark$ & & $\checkmark$ \\
\hline 35 & s16 & & & & \\
\hline 36 & s16 & & & & \\
\hline 37 & s17 & $\checkmark$ & & $\checkmark$ & \\
\hline
\end{tabular}


Passage A11

\begin{tabular}{|c|c|c|c|c|c|}
\hline \multirow[t]{2}{*}{$\begin{array}{l}\text { IU Label } \\
\#\end{array}$} & \multirow{2}{*}{$\begin{array}{l}\text { Sentence IU is } \\
\text { found in } \\
\#\end{array}$} & \multicolumn{4}{|c|}{ Existence of IU in Student Free Recall } \\
\hline & & S7 & S9 & S12 & S13 \\
\hline 1 & s1 & $\checkmark$ & $\checkmark$ & $\checkmark$ & \\
\hline 2 & s1 & $\checkmark$ & $\checkmark$ & $\checkmark$ & \\
\hline 3 & s1 & $\checkmark$ & $\checkmark$ & $\checkmark$ & \\
\hline 4 & s2 & & $\checkmark$ & $\checkmark$ & $\checkmark$ \\
\hline 5 & s3 & $\checkmark$ & $\checkmark$ & & \\
\hline 6 & s4 & $\checkmark$ & $\checkmark$ & & \\
\hline 7 & s4 & $\checkmark$ & $\checkmark$ & & \\
\hline 8 & s4 & & & & \\
\hline 9 & s4 & & $\checkmark$ & & \\
\hline 10 & s5 & & $\checkmark$ & & \\
\hline 11 & s5 & $\checkmark$ & & & \\
\hline 12 & s6 & & & $\checkmark$ & \\
\hline 13 & s6 & & & & \\
\hline 14 & s6 & & & & \\
\hline 15 & s7 & & & $\checkmark$ & \\
\hline 16 & s7 & & & & \\
\hline 17 & s8 & $\checkmark$ & & & $\checkmark$ \\
\hline 18 & s9 & & & & \\
\hline 19 & s10 & & & $\checkmark$ & \\
\hline 20 & s10 & & & $\checkmark$ & \\
\hline 21 & s10 & & & & \\
\hline 22 & s11 & & & & \\
\hline 23 & s12 & & & & \\
\hline 24 & s13 & & & & \\
\hline 25 & s13 & & & & \\
\hline 26 & s13 & & & & \\
\hline 27 & s14 & & & $\checkmark$ & \\
\hline 28 & s14 & $\checkmark$ & & & \\
\hline 29 & s14 & & & & \\
\hline 30 & s15 & & & & \\
\hline 31 & s16 & & $\checkmark$ & & \\
\hline 32 & s17 & & $\checkmark$ & & \\
\hline 33 & s18 & $\checkmark$ & & & \\
\hline 34 & s18 & & & & \\
\hline
\end{tabular}




\section{Appendix 8: Map of frequency of IU recall patterns within sentences in Course A (Passages A7-A11)}

Passage A7

KEY

\begin{tabular}{|l|l|}
\hline & Exists in ALL 4 student recalls \\
\hline & Exists in 3 out of 4 student recalls \\
\hline & Exists in 2 out of 4 student recalls \\
\hline & Exists in 1 out of 4 student recalls \\
\hline $\begin{array}{l}\text { Does NOT exist in ANY student } \\
\text { recalls }\end{array}$ \\
\hline
\end{tabular}

\begin{tabular}{|c|c|c|}
\hline & $\begin{array}{l}\text { Sentence } \\
\text { length } \\
\text { (no. of } \\
\text { words) }\end{array}$ & \\
\hline s1 & 24 & $\begin{array}{l}\text { In his book The Age of Unreason, British scholar and consultant } \\
\text { Charles Handy discusses dramatic new developments in the world } \\
\text { of work and careers. }\end{array}$ \\
\hline s2 & 13 & $\begin{array}{l}\text { Specifically, Handy says: 'The times are changing and we must } \\
\text { change with them. }\end{array}$ \\
\hline s3 & 13 & Each of us should take this advice and take charge of our careers. \\
\hline s4 & 18 & $\begin{array}{l}\text { It should be said, however, that the best companies do not leave } \\
\text { career development just to individual employees. }\end{array}$ \\
\hline s5 & 32 & $\begin{array}{l}\text { A study of Australian companies found that the best employers } \\
\text { emphasized the career development of their people in an effort to } \\
\text { keep employees satisfied with their jobs and their own personal } \\
\text { development. }\end{array}$ \\
\hline s6 & 17 & $\begin{array}{l}\text { The best employers were more likely to implement one-on-one } \\
\text { mentoring and leadership development programs than other } \\
\text { organisations. }\end{array}$ \\
\hline s7 & 33 & $\begin{array}{l}\text { They were committed to building a tripartite commitment to career } \\
\text { development, involving the organisation as well as each } \\
\text { individual's manager or supervisor; and in addition employees } \\
\text { were involved in their own development plans. }\end{array}$ \\
\hline s8 & 21 & $\begin{array}{l}\text { Formally defined, a career is a sequence of jobs and work pursuits } \\
\text { that constitute what a person does for a living. }\end{array}$ \\
\hline s9 & 15 & $\begin{array}{l}\text { For many of us, a career begins on an anticipatory basis with our } \\
\text { formal education. }\end{array}$ \\
\hline s10 & 27 & $\begin{array}{l}\text { From there it progresses into an initial job choice and any number } \\
\text { of subsequent choices that may involve changes in task } \\
\text { assignments, employing organisations and even occupations. }\end{array}$ \\
\hline s11 & 14 & A career path is a sequence of jobs held over time during a career. \\
\hline
\end{tabular}




\begin{tabular}{|l|l|l|}
\hline s12 & 20 & $\begin{array}{l}\text { Career paths vary between those that are pursued internally with } \\
\text { the same employers and those pursued externally among various } \\
\text { employers. }\end{array}$ \\
\hline s13 & 29 & $\begin{array}{l}\text { Whereas many organisations place great emphasis on making } \\
\text { long-term career opportunities available to their employees, } \\
\text { Handy's view of the future is that external career paths will be } \\
\text { increasingly important. }\end{array}$ \\
\hline s14 & 16 & $\begin{array}{l}\text { A career is a sequence of jobs that constitute what a person does } \\
\text { for a living. }\end{array}$ \\
\hline
\end{tabular}




\section{Passage A8}

\section{KEY}

\begin{tabular}{|l|l|}
\hline & Exists in ALL 4 student recalls \\
\hline & Exists in 3 out of 4 student recalls \\
\hline & Exists in 2 out of 4 student recalls \\
\hline & Exists in 1 out of 4 student recalls \\
\hline $\begin{array}{l}\text { Does NOT exist in ANY student } \\
\text { recalls }\end{array}$ \\
\hline
\end{tabular}

\begin{tabular}{|l|l|l|}
\hline & $\begin{array}{l}\text { Sentence } \\
\text { length } \\
\text { (no. of } \\
\text { words) }\end{array}$ & $\begin{array}{l}\text { Erin Brokovich, a legal clerk, was instrumental in constructing a } \\
\text { case against the Pacific Gas \& Electric company in California for } \\
\text { contaminating drinking water. }\end{array}$ \\
\hline s1 & 24 & $\begin{array}{l}\text { Jeffrey Wigland exposed his US tobacco company's practice of } \\
\text { internationally manipulating the effect of nicotine in cigarettes. }\end{array}$ \\
\hline s2 & 17 & $\begin{array}{l}\text { Toni Hoffman, a nurse in charge of the Intensive Care Unit at } \\
\text { Bundaberg Base Hospital in Queensland, tried for two years to } \\
\text { raise concerns about the lethal activities of a surgeon, who is now } \\
\text { accused of murder. }\end{array}$ \\
\hline s3 & 37 & $\begin{array}{l}\text { Detective Sergeant Simon Illingworth spent four years working for } \\
\text { Police Internal Affairs in Victoria, weeding out crooked police and } \\
\text { putting his life at risk. }\end{array}$ \\
\hline s4 & 24 & $\begin{array}{l}\text { They were whistleblowers, people who expose the misdeeds of } \\
\text { others in organisations in order to preserve the ethical standards } \\
\text { and protect against wasteful, harmful or illegal acts. }\end{array}$ \\
\hline s5 & 27 & $\begin{array}{l}\text { Whistleblowers face the risks of impaired career progress and } \\
\text { other forms of organisational retaliation, up to and including } \\
\text { dismissal. }\end{array}$ \\
\hline s6 & 19 & $\begin{array}{l}\text { Many workers become whistleblowers unintentionally when } \\
\text { reporting workplace fraud, corruption or maladministration. }\end{array}$ \\
\hline s10 & 13 & $\begin{array}{l}\text { Today, federal and state laws in countries such as Australia and } \\
\text { New Zealand increasingly offer whistleblowers some defence } \\
\text { against 'retaliatory discharge'. }\end{array}$ \\
\hline s11 & 18 & $\begin{array}{l}\text { But although signs indicate that the courts are growing supportive } \\
\text { of whistleblowers, legal protection for them can still be } \\
\text { inadequate. }\end{array}$ \\
\hline s12 & 40 & $\begin{array}{l}\text { Laws vary from state to state, and federal laws mainly protect } \\
\text { government workers. } \\
\text { Furthermore, even with legal protection, potential whistleblowers } \\
\text { may find it hard to expose unethical behaviour in the workplace. }\end{array}$ \\
\hline Some organisational barriers to whistleblowing include a strict \\
\hline
\end{tabular}




\begin{tabular}{|l|l|l|}
\hline & & $\begin{array}{l}\text { chain of command that makes it hard to bypass the boss, strong } \\
\text { work group identities that encourage loyalty and self-censorship, } \\
\text { and ambiguous priorities that make it hard to distinguish right } \\
\text { from wrong. }\end{array}$ \\
\hline s13 & 27 & $\begin{array}{l}\text { In the attempt to remove these and other blocks to the exposure of } \\
\text { unethical behaviours, some organisations have formally appointed } \\
\text { staff members to serve as 'ethical advisors'. }\end{array}$ \\
\hline s14 & 11 & $\begin{array}{l}\text { Others have set up formal staff units to process reported } \\
\text { infractions. }\end{array}$ \\
\hline s15 & 28 & $\begin{array}{l}\text { One novel proposal goes so far as to suggest the convening of } \\
\text { moral quality circles to help create shared commitments for people } \\
\text { to work at their moral best. }\end{array}$ \\
\hline s16 & 8 & Whistleblowers expose the misdeeds of others in organisations. \\
\hline
\end{tabular}




\section{Passage A9}

\section{KEY}

\begin{tabular}{|l|l|}
\hline & Exists in ALL 4 student recalls \\
\hline & Exists in 3 out of 4 student recalls \\
\hline & Exists in 2 out of 4 student recalls \\
\hline & Exists in 1 out of 4 student recalls \\
\hline $\begin{array}{l}\text { Does NOT exist in ANY student } \\
\text { recalls }\end{array}$ \\
\hline
\end{tabular}

\begin{tabular}{|c|c|c|}
\hline & $\begin{array}{l}\text { Sentence } \\
\text { length } \\
\text { (no. of } \\
\text { words) }\end{array}$ & \\
\hline s1 & 8 & The nature of the task is always important. \\
\hline s2 & 24 & $\begin{array}{l}\text { It affects how well a team can focus its efforts and how intense the } \\
\text { group process needs to be to get the job done. }\end{array}$ \\
\hline s3 & 14 & $\begin{array}{l}\text { Clearly defined tasks make it easier for team members to focus } \\
\text { their work efforts. }\end{array}$ \\
\hline s4 & 13 & $\begin{array}{l}\text { Complex tasks require more information exchange and intense } \\
\text { interaction than do simpler tasks. }\end{array}$ \\
\hline s5 & 20 & $\begin{array}{l}\text { The organisational setting can also affect how team members } \\
\text { relate to one another and apply their skills towards task } \\
\text { accomplishment. }\end{array}$ \\
\hline s6 & 23 & $\begin{array}{l}\text { A key issue is the amount of support provided in terms of } \\
\text { information, material resources, technology, organisational } \\
\text { structures, available rewards and spatial arrangements. }\end{array}$ \\
\hline s7 & 12 & $\begin{array}{l}\text { Increasingly, for example, organisations are being architecturally } \\
\text { designed to directly facilitate teamwork. }\end{array}$ \\
\hline s8 & 27 & $\begin{array}{l}\text { At St Luke's Advertising Agency in London, the traditional office } \\
\text { environment has been changed in favour of a long desk in the } \\
\text { centre of a large room. }\end{array}$ \\
\hline s9 & 24 & $\begin{array}{l}\text { The desk has facilities for laptop computer connection, and } \\
\text { provides a central focus at which open communication and the } \\
\text { flow of ideas are encouraged. }\end{array}$ \\
\hline s10 & 48 & $\begin{array}{l}\text { There are some benches around the desk, as well as meeting } \\
\text { rooms nearby; however, the company aims at eliminating the } \\
\text { sense of hierarchy normally associated with the size, location and } \\
\text { layout of offices and instead promoting the type of creative } \\
\text { thinking for which St Luke's has become famous. }\end{array}$ \\
\hline s11 & 12 & $\begin{array}{l}\text { Team size affects how members work together, handle } \\
\text { disagreements and reach agreements. }\end{array}$ \\
\hline s12 & 16 & $\begin{array}{l}\text { The number of potential interactions increases as teams increase in } \\
\text { size and communications become more congested. }\end{array}$ \\
\hline
\end{tabular}




\begin{tabular}{|c|c|c|}
\hline s13 & 20 & $\begin{array}{l}\text { Teams larger than about six or seven members can be difficult to } \\
\text { manage for the purpose of creative problem solving. }\end{array}$ \\
\hline s14 & 18 & $\begin{array}{l}\text { When voting is required, teams with odd numbers of members are } \\
\text { often preferred so as to prevent 'ties'. }\end{array}$ \\
\hline s15 & 8 & In all teams, the membership characteristics are important. \\
\hline s16 & 17 & $\begin{array}{l}\text { Teams must have members with the right abilities or skill mix to } \\
\text { master and perform tasks well. }\end{array}$ \\
\hline s17 & 21 & $\begin{array}{l}\text { They must also have values, personalities and diversity } \\
\text { appropriate to the task that are sufficiently compatible for } \\
\text { everyone to work together. }\end{array}$ \\
\hline
\end{tabular}


Passage A10

KEY

\begin{tabular}{|l|l|}
\hline & Exists in ALL 4 student recalls \\
\hline & Exists in 3 out of 4 student recalls \\
\hline & Exists in 2 out of 4 student recalls \\
\hline & Exists in 1 out of 4 student recalls \\
\hline $\begin{array}{l}\text { Does NOT exist in ANY student } \\
\text { recalls }\end{array}$ \\
\hline
\end{tabular}

\begin{tabular}{|c|c|c|}
\hline & $\begin{array}{l}\text { Sentence } \\
\text { length } \\
\text { (no. of } \\
\text { words) }\end{array}$ & \\
\hline s1 & 13 & $\begin{array}{l}\text { Resources and customers are two critical elements in the open- } \\
\text { systems view of organisations. }\end{array}$ \\
\hline s2 & 19 & $\begin{array}{l}\text { For an organisation to perform well, resources must be put to good } \\
\text { use and customers must be well served. }\end{array}$ \\
\hline s3 & 11 & The notion of value creation is very important in this context. \\
\hline s4 & 37 & $\begin{array}{l}\text { If operations add value to the original cost of the resource inputs, } \\
\text { then a business organisation can earn a profit - that is, sell a } \\
\text { product for more than the cost of making it (e.g. fast-food } \\
\text { restaurant meals). }\end{array}$ \\
\hline s5 & 28 & $\begin{array}{l}\text { A not-for-profit organisation can add wealth to society - that is, } \\
\text { provide a public service that is worth more than its cost (e.g. fire } \\
\text { prevention services in a community). }\end{array}$ \\
\hline s6 & 31 & $\begin{array}{l}\text { Value is created when an organisation's resources are used in the } \\
\text { right way and at the right time and at minimum cost to create high } \\
\text { quality goods and services for customers. }\end{array}$ \\
\hline s7 & 9 & The best organisations use a variety of performance measures. \\
\hline s8 & 16 & $\begin{array}{l}\text { On the customer side, high-performing organisations measure } \\
\text { customer satisfaction and loyalty, as well as market share. }\end{array}$ \\
\hline s9 & 14 & $\begin{array}{l}\text { On the employee side, they measure retention, career } \\
\text { development, job satisfaction and related issues. }\end{array}$ \\
\hline s10 & 29 & $\begin{array}{l}\text { One of the most common indicators of organisational performance } \\
\text { overall is productivity, a summary measure of the quantity and } \\
\text { quality of work performance with resource use taken into account. }\end{array}$ \\
\hline s11 & 16 & $\begin{array}{l}\text { Productivity can be measured at the individual and group levels as } \\
\text { well as at organisational levels. }\end{array}$ \\
\hline s12 & 14 & $\begin{array}{l}\text { Figure } 1.2 \text { links productivity with two terms commonly used in } \\
\text { management - effectiveness and efficiency. }\end{array}$ \\
\hline s13 & 11 & $\begin{array}{l}\text { Performance effectiveness is a measure of task output or goal } \\
\text { accomplishment. }\end{array}$ \\
\hline s14 & 35 & $\begin{array}{l}\text { If you are working in the manufacturing area of a computer firm, } \\
\text { for example, performance effectiveness may mean that you meet a }\end{array}$ \\
\hline
\end{tabular}




\begin{tabular}{|l|l|l|}
\hline & & $\begin{array}{l}\text { daily production target in terms of the quantity and quality of } \\
\text { keyboards assembled. }\end{array}$ \\
\hline s15 & 26 & $\begin{array}{l}\text { By so doing, you allow the company as a whole to maintain its } \\
\text { production schedule and meet customer demands for timely } \\
\text { delivery and high quality products. }\end{array}$ \\
\hline s16 & 13 & $\begin{array}{l}\text { Productivity is the quantity and quality of work performance, with } \\
\text { resource use considered. }\end{array}$ \\
\hline s17 & 11 & $\begin{array}{l}\text { Performance effectiveness is an output measure of task or goal } \\
\text { accomplishment. }\end{array}$ \\
\hline
\end{tabular}




\section{Passage A11}

\section{KEY}

\begin{tabular}{|l|l|}
\hline & Exists in ALL 4 student recalls \\
\hline & Exists in 3 out of 4 student recalls \\
\hline & Exists in 2 out of 4 student recalls \\
\hline & Exists in 1 out of 4 student recalls \\
\hline $\begin{array}{l}\text { Does NOT exist in ANY student } \\
\text { recalls }\end{array}$ \\
\hline
\end{tabular}

\begin{tabular}{|c|c|c|}
\hline & $\begin{array}{l}\text { Sentence } \\
\text { length } \\
\text { (no. of } \\
\text { words) }\end{array}$ & \\
\hline s1 & 18 & $\begin{array}{l}\text { Formally defined, a system is a collection of interrelated parts that } \\
\text { function together to achieve a common purpose. }\end{array}$ \\
\hline s2 & 10 & A subsystem is a smaller component of a larger system. \\
\hline s3 & 14 & $\begin{array}{l}\text { One of the earliest management writers to adopt a systems } \\
\text { perspective was Chester Barnard. }\end{array}$ \\
\hline s4 & 19 & $\begin{array}{l}\text { His } 1938 \text { groundbreaking book Functions of the Executive was } \\
\text { based on years of experience as a telephone company executive. }\end{array}$ \\
\hline s5 & 18 & $\begin{array}{l}\text { Barnard described organisations as cooperative systems in which } \\
\text { the contributions of individuals are integrated for a common } \\
\text { purpose. }\end{array}$ \\
\hline s6 & 9 & $\begin{array}{l}\text { Importantly, Barnard considered this cooperation conscious, } \\
\text { deliberate and purposeful. }\end{array}$ \\
\hline s7 & 16 & $\begin{array}{l}\text { It was the job of the executives, or managers, through } \\
\text { communication to make this cooperation happen. }\end{array}$ \\
\hline s8 & 9 & $\begin{array}{l}\text { Systems thinking continues to influence management and practice } \\
\text { today. }\end{array}$ \\
\hline s9 & 7 & One application is described in Figure 4.4. \\
\hline s10 & 29 & $\begin{array}{l}\text { This figure first depicts the larger organisation as an open system } \\
\text { that interacts with its environment in the continual process of } \\
\text { transforming inputs from suppliers into outputs for customers. }\end{array}$ \\
\hline s11 & 11 & $\begin{array}{l}\text { Within the organisation any number of critical subsystems can be } \\
\text { described. }\end{array}$ \\
\hline s12 & 13 & $\begin{array}{l}\text { In the figure, the operations and service management systems are a } \\
\text { central point. }\end{array}$ \\
\hline s13 & 23 & $\begin{array}{l}\text { They facilitate the interactions between other subsystems - such as } \\
\text { purchasing, accounting, sales and information - that are essential } \\
\text { to the work of the organisation. }\end{array}$ \\
\hline s14 & 32 & $\begin{array}{l}\text { Importantly, and as suggested by Barnard, high performance by } \\
\text { the organisation as a whole occurs only when each subsystem } \\
\text { performs its task well and works well in cooperation with the other } \\
\text { subsystems. }\end{array}$ \\
\hline
\end{tabular}




\begin{tabular}{|l|l|l|}
\hline s15 & 15 & $\begin{array}{l}\text { It is the job of managers throughout the organisation to make this } \\
\text { coordinated action possible. }\end{array}$ \\
\hline s16 & 13 & $\begin{array}{l}\text { A system is a collection of interrelated parts working together for } \\
\text { a purpose. }\end{array}$ \\
\hline s17 & 10 & A subsystem is a smaller component of a larger system. \\
\hline s18 & 11 & $\begin{array}{l}\text { Open systems transform resource inputs from the environment } \\
\text { into product outputs. }\end{array}$ \\
\hline
\end{tabular}




\section{Appendix 9: IU analysis of Course A text sample Passages A6-A11}

Passage A6

\begin{tabular}{|c|c|c|}
\hline \# & Sentence & Idea Unit \\
\hline 1 & S1 & $\begin{array}{l}\text { Communication and related interpersonal skills must be at the forefront of } \\
\text { any attempt to develop managerial and leadership expertise. }\end{array}$ \\
\hline 2 & S2 & $\begin{array}{l}\text { Among these essential skills, the ability to deal with interpersonal conflicts is } \\
\text { critical. }\end{array}$ \\
\hline 3 & S3 & Conflict can be formally defined \\
\hline 4 & S3 & $\begin{array}{l}\text { Conflict is a disagreement between people on substantive or emotional } \\
\text { issues. }\end{array}$ \\
\hline 5 & S4 & $\begin{array}{l}\text { Managers and leaders spend a lot of time dealing with conflicts of various } \\
\text { forms. }\end{array}$ \\
\hline 6 & S5, S16 & Substantive conflicts involve disagreements over such things as goals. \\
\hline 7 & S5, S16 & $\begin{array}{l}\text { Substantive conflicts involve disagreements over such things as the allocation } \\
\text { of resources. }\end{array}$ \\
\hline 8 & S5, S16 & $\begin{array}{l}\text { Substantive conflicts involve disagreements over such things as the } \\
\text { distribution of rewards. }\end{array}$ \\
\hline 9 & S5, S16 & $\begin{array}{l}\text { Substantive conflicts involve disagreements over such things as policies and } \\
\text { procedures. }\end{array}$ \\
\hline 10 & S5, S16 & $\begin{array}{l}\text { Substantive conflicts involve disagreements over such things as job } \\
\text { assignments. }\end{array}$ \\
\hline 11 & S6, S17 & Emotional conflicts result from feelings of anger \\
\hline 12 & S6, S17 & Emotional conflicts result from feelings of distrust \\
\hline 13 & S6, S17 & Emotional conflicts result from feelings of dislike \\
\hline 14 & S6, S17 & Emotional conflicts result from feelings of fear \\
\hline 15 & S6, S17 & Emotional conflicts result from feelings of resentment \\
\hline 16 & S6, S17 & Emotional conflicts result from feelings of personality clashes. \\
\hline 17 & S7 & Both forms of conflict can cause problems in the workplace. \\
\hline 18 & S8 & But when managed well, they can be helpful in promoting high performance. \\
\hline 19 & S8 & But when managed well, they can be helpful in promoting creativity. \\
\hline 20 & S8 & But when managed well, they can be helpful in promoting innovation. \\
\hline 21 & S9 & These are the Consequences of conflict \\
\hline 22 & S10 & $\begin{array}{l}\text { Whether or not conflict benefits people and organisations depends on two } \\
\text { factors }\end{array}$ \\
\hline 23 & S10 & $\begin{array}{l}\text { Whether or not conflict benefits people and organisations depends on the } \\
\text { intensity of the conflict }\end{array}$ \\
\hline 24 & S10 & $\begin{array}{l}\text { Whether or not conflict benefits people and organisations depends on how } \\
\text { well the conflict is managed. }\end{array}$ \\
\hline 25 & S11 & $\begin{array}{l}\text { The inverted ' } U \text { ' curve in Figure } 17.4 \text { shows that conflict of moderate } \\
\text { intensity can be good for performance. }\end{array}$ \\
\hline 26 & S12 & Functional conflict is constructive conflict. \\
\hline 27 & S12 & This conflict stimulates people to greater work efforts. \\
\hline 28 & S12 & This conflict stimulates people to greater cooperation. \\
\hline 29 & S12 & This conflict stimulates people to greater creativity. \\
\hline 30 & S13 & Dysfunctional conflict is destructive conflict. \\
\hline 31 & S13 & This type of conflict occurs at very low intensities. \\
\hline
\end{tabular}




\begin{tabular}{|l|l|l|}
\hline 32 & S13 & This type of conflict occurs at very high intensities. \\
\hline 33 & S14 & Too much conflict interferes with other more task-relevant activities. \\
\hline 34 & S14 & Too little conflict may promote complacency. \\
\hline 35 & S14 & Too little conflict may promote the loss of a creative, high-performance edge. \\
\hline 36 & S15 & Conflict is a disagreement over issues of substance. \\
\hline 37 & S15 & Conflict is an emotional antagonism. \\
\hline 38 & S18 & Functional conflict is constructive. \\
\hline 39 & S18 & Functional conflict helps task performance. \\
\hline 40 & S19 & Dysfunctional conflict is destructive. \\
\hline 41 & S19 & Dysfunctional conflict hurts task performance. \\
\hline
\end{tabular}


Passage A7

\begin{tabular}{|c|c|c|}
\hline \# & $\begin{array}{l}\text { Sentence } \\
\#\end{array}$ & Simple sentences \\
\hline 1 & S1 & Charles Handy is $a$ British scholar and consultant. \\
\hline 2 & S1 & Charles Handy wrote a book. \\
\hline 3 & $\mathrm{~S} 1$ & The book was called The Age of Unreason \\
\hline 4 & $\mathrm{~S} 1$ & $\begin{array}{l}\text { In his book, Handy discusses dramatic new developments in the } \\
\text { world of work and careers. }\end{array}$ \\
\hline 5 & $\mathrm{~S} 2$ & Handy specifically says that times are changing. \\
\hline 6 & $\mathrm{~S} 2$ & Handy sys that we must change with them. \\
\hline 7 & S3 & Each of us should take this advice. \\
\hline 8 & $\mathrm{~S} 3$ & Each of us should take charge of our careers. \\
\hline 9 & $\mathrm{~S} 4$ & $\begin{array}{l}\text { However, the best companies do not leave career development just } \\
\text { to individual employees. }\end{array}$ \\
\hline 10 & S5 & There was a study of Australian companies. \\
\hline 11 & S5 & $\begin{array}{l}\text { The study found that the best employers emphasized the career } \\
\text { development of their people. }\end{array}$ \\
\hline 12 & S5 & $\begin{array}{l}\text { They did this in an effort to keep employees satisfied with their } \\
\text { jobs and personal development. }\end{array}$ \\
\hline 13 & S6 & $\begin{array}{l}\text { The best employers were more likely to implement one-on-one } \\
\text { mentoring than other organisations. }\end{array}$ \\
\hline 14 & S6 & $\begin{array}{l}\text { The best employers were more likely to implement leadership } \\
\text { development programs than other organisations. }\end{array}$ \\
\hline 15 & S7 & $\begin{array}{l}\text { They were committed to building a tripartite commitment to } \\
\text { career development. }\end{array}$ \\
\hline 16 & S7 & $\begin{array}{l}\text { This commitment involved the organisation as well as each } \\
\text { individual's manager or supervisor. }\end{array}$ \\
\hline 17 & S7 & $\begin{array}{l}\text { In addition, employees were involved in their own development } \\
\text { plans. }\end{array}$ \\
\hline 18 & S8 & Career can be formally defined. \\
\hline 19 & S8 & $\begin{array}{l}\text { This definition is the sequence of jobs and work pursuits that } \\
\text { constitute what a person does for a living. }\end{array}$ \\
\hline 20 & S9 & $\begin{array}{l}\text { For many of us, a career begins on an anticipatory basis with our } \\
\text { formal education. }\end{array}$ \\
\hline 21 & S10 & From there it progresses into an initial job choice. \\
\hline 22 & S10 & It then progresses into any number of subsequent choices. \\
\hline 23 & S10 & $\begin{array}{l}\begin{array}{l}\text { These subsequent choices may involve changes in task } \\
\text { assignments. }\end{array} \\
\end{array}$ \\
\hline 24 & S10 & $\begin{array}{l}\text { These subsequent choices may involve changes in employing } \\
\text { organisations. }\end{array}$ \\
\hline 25 & S10 & These subsequent choices may involve changes in occupations. \\
\hline 26 & $\mathrm{~S} 11$ & A career path is a sequence of jobs. \\
\hline 27 & S11 & These jobs are held over time during a career. \\
\hline 28 & $\mathrm{~S} 12$ & Career paths vary. \\
\hline
\end{tabular}




\begin{tabular}{|l|l|l|}
\hline 29 & S12 & $\begin{array}{l}\text { They vary between those that are pursued internally with the same } \\
\text { employers and those pursued externally among various employers. }\end{array}$ \\
\hline 30 & S13 & $\begin{array}{l}\text { Many organisations place great emphasis on making long-term } \\
\text { career opportunities available to their employees. }\end{array}$ \\
\hline 31 & S13 & $\begin{array}{l}\text { Whereas Handy's view of the future is that external career paths } \\
\text { will be increasingly important. }\end{array}$ \\
\hline 32 & S14 & $\begin{array}{l}\text { A career is a sequence of jobs that constitute what a person does } \\
\text { for a living. }\end{array}$ \\
\hline
\end{tabular}


Passage 8

\begin{tabular}{|c|c|c|}
\hline \# & $\begin{array}{l}\text { Sentence } \\
\#\end{array}$ & Idea Unit \\
\hline 1 & S1 & Erin Brokovich is a legal clerk. \\
\hline 2 & S1 & $\begin{array}{l}\text { Erin Brokovich was instrumental in constructing a case against the } \\
\text { Pacific Gas \& Electric company in California for contaminating } \\
\text { drinking water. }\end{array}$ \\
\hline 3 & $\mathrm{~S} 2$ & $\begin{array}{l}\text { Jeffrey Wigland exposed his US tobacco company's practice of } \\
\text { internationally manipulating the effect of nicotine in cigarettes. }\end{array}$ \\
\hline 4 & S3 & $\begin{array}{l}\text { Toni Hoffman is a nurse in charge of the Intensive Care Unit at } \\
\text { Bundaberg Base Hospital in Queensland, }\end{array}$ \\
\hline 5 & S3 & $\begin{array}{l}\text { Toni Hoffman tried for two years to raise concerns about the lethal } \\
\text { activities of a surgeon. }\end{array}$ \\
\hline 6 & S3 & This surgeon is now accused of murder. \\
\hline 7 & S4 & $\begin{array}{l}\text { Detective Sergeant Simon Illingworth spent four years working for } \\
\text { Police Internal Affairs in Victoria. }\end{array}$ \\
\hline 8 & S4 & He weeded out crooked police. \\
\hline 9 & S4 & He put his life at risk. \\
\hline 10 & S5 & They were whistleblowers, \\
\hline 11 & S5 & $\begin{array}{l}\text { Whistleblowers are people who expose the misdeeds of others in } \\
\text { organisations. }\end{array}$ \\
\hline 12 & S5 & They do this in order to preserve the ethical standards. \\
\hline 13 & S5 & They do this in order to protect against wasteful acts. \\
\hline 14 & S5 & They do this in order to protect against harmful acts. \\
\hline 15 & S5 & They do this in order to protect against illegal acts. \\
\hline 16 & S6 & Whistleblowers face the risks of impaired career progress. \\
\hline 17 & S6 & Whistleblowers face other forms of organisational retaliation. \\
\hline 18 & S6 & These other forms go up to and including dismissal. \\
\hline 19 & S7 & Many workers become whistleblowers unintentionally. \\
\hline 20 & S7 & This occurs when reporting workplace fraud. \\
\hline 21 & S7 & This occurs when reporting corruption. \\
\hline 22 & S7 & This occurs when reporting maladministration. \\
\hline 23 & S8 & $\begin{array}{l}\text { Today, federal and state laws in countries such as Australia and New } \\
\text { Zealand increasingly offer whistleblowers some defence against } \\
\text { 'retaliatory discharge'. }\end{array}$ \\
\hline 24 & S9 & $\begin{array}{l}\text { Signs indicate that the courts are growing supportive of } \\
\text { whistleblowers, }\end{array}$ \\
\hline 25 & S9 & $\begin{array}{l}\text { But although this is so, legal protection for them can still be } \\
\text { inadequate. }\end{array}$ \\
\hline 26 & S10 & Laws vary from state to state. \\
\hline 27 & S10 & And federal laws mainly protect government workers. \\
\hline 28 & S11 & $\begin{array}{l}\text { Furthermore, even with legal protection, potential whistleblowers } \\
\text { may find it hard to expose unethical behaviour in the workplace. }\end{array}$ \\
\hline 29 & S12 & There are organisational barriers to whistleblowing. \\
\hline 30 & S12 & Some of these barriers include a strict chain of command. \\
\hline
\end{tabular}




\begin{tabular}{|c|c|c|}
\hline 31 & S12 & A strict chain of command makes it hard to bypass the boss. \\
\hline 32 & S12 & Strong work group identities are a barrier to whistleblowing. \\
\hline 33 & S12 & Strong work group identities encourage loyalty and self-censorship. \\
\hline 34 & S12 & Ambiguous priorities are a barrier to whistleblowing. \\
\hline 35 & S12 & Ambiguous priorities make it hard to distinguish right from wrong. \\
\hline 36 & S13 & $\begin{array}{l}\text { Some organisations have formally appointed staff members to serve } \\
\text { as 'ethical advisors'. }\end{array}$ \\
\hline 37 & S13 & $\begin{array}{l}\text { They have done this in the attempt to remove these and other blocks } \\
\text { to the exposure of unethical behaviours, }\end{array}$ \\
\hline 38 & S14 & Others have set up formal staff units. \\
\hline 39 & S14 & These units will process reported infractions. \\
\hline 40 & S15 & $\begin{array}{l}\text { One novel proposal goes so far as to suggest the convening of moral } \\
\text { quality circles }\end{array}$ \\
\hline 41 & S15 & These moral quality circles will help create shared commitments. \\
\hline 42 & S15 & These shared commitments help people to work at their moral best. \\
\hline 43 & S16 & Whistleblowers expose the misdeeds of others in organisations. \\
\hline
\end{tabular}


Passage A9

\begin{tabular}{|c|c|c|}
\hline 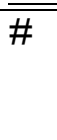 & $\begin{array}{l}\text { Sen- } \\
\text { tence } \\
\#\end{array}$ & Idea Units \\
\hline 1 & S1 & The nature of the task is always important. \\
\hline 2 & S2 & $\begin{array}{l}\text { The nature of the task affects how well a team can focus its } \\
\text { efforts }\end{array}$ \\
\hline 3 & S2 & $\begin{array}{l}\text { The nature of the task affects how intense the group process } \\
\text { needs to be to get the job done }\end{array}$ \\
\hline 4 & S3 & $\begin{array}{l}\text { Clearly defined tasks make it easier for team members to focus } \\
\text { their work efforts. }\end{array}$ \\
\hline 5 & S4 & $\begin{array}{l}\text { Complex tasks require more information exchange than do } \\
\text { simpler tasks. }\end{array}$ \\
\hline 6 & S4 & $\begin{array}{l}\text { Complex tasks require more intense interaction than do simpler } \\
\text { tasks. }\end{array}$ \\
\hline 7 & S5 & $\begin{array}{l}\text { The organisational setting can also affect how team members } \\
\text { relate to one another. }\end{array}$ \\
\hline 8 & S5 & $\begin{array}{l}\text { The organisational setting can also affect how team members } \\
\text { apply their skills towards task accomplishment. }\end{array}$ \\
\hline 9 & S6 & A key issue is the amount of support provided \\
\hline 10 & S6 & $\begin{array}{l}\text { The support is provided in terms of information, material } \\
\text { resources, technology, organisational structures, available } \\
\text { rewards and spatial arrangements. }\end{array}$ \\
\hline 11 & S7 & $\begin{array}{l}\text { Increasingly, for example, organisations are being } \\
\text { architecturally designed to directly facilitate teamwork }\end{array}$ \\
\hline 12 & S8 & $\begin{array}{l}\text { At St Luke's Advertising Agency in London, the traditional } \\
\text { office environment has been changed. }\end{array}$ \\
\hline 13 & S8 & $\begin{array}{l}\text { The traditional office environment has been changed in favour } \\
\text { of a long desk in the centre of a large room. }\end{array}$ \\
\hline 14 & S9 & The desk has facilities for laptop computer connection. \\
\hline 15 & S9 & The desk provides a central focus. \\
\hline 16 & S9 & $\begin{array}{l}\text { Open communication and the flow of ideas are encouraged at } \\
\text { the desk. }\end{array}$ \\
\hline 17 & S10 & There are some benches around the desk. \\
\hline 18 & S10 & There are meeting rooms nearby as well. \\
\hline 19 & S10 & $\begin{array}{l}\text { However, the company aims at eliminating the sense of } \\
\text { hierarchy }\end{array}$ \\
\hline 20 & S10 & $\begin{array}{l}\text { This sense of hierarchy is normally associated with the size, } \\
\text { location and layout of offices }\end{array}$ \\
\hline 21 & S10 & Instead, the company promotes creative thinking \\
\hline 22 & S10 & St Luke's has become famous for this creative thinking. \\
\hline 23 & S11 & Team size affects how members work together. \\
\hline 24 & S11 & Team size affects how members handle disagreements. \\
\hline 25 & S11 & Team size affects how members reach agreements. \\
\hline 26 & S12 & $\begin{array}{l}\text { The number of potential interactions increases as teams } \\
\text { increase in size. }\end{array}$ \\
\hline
\end{tabular}




\begin{tabular}{|c|c|c|}
\hline 27 & S12 & $\begin{array}{l}\text { The number of potential interactions increases as } \\
\text { communications become more congested. }\end{array}$ \\
\hline 28 & S13 & $\begin{array}{l}\text { Teams larger than about six or seven members can be difficult } \\
\text { to manage for the purpose of creative problem solving. }\end{array}$ \\
\hline 29 & S14 & $\begin{array}{l}\text { When voting is required, teams with odd numbers of members } \\
\text { are often preferred so as to prevent 'ties' }\end{array}$ \\
\hline 30 & S15 & In all teams, the membership characteristics are important. \\
\hline 31 & S16 & Teams must have members with the right abilities or skill mix. \\
\hline 32 & S16 & $\begin{array}{l}\text { These abilities or skills should be the right ones to master and } \\
\text { perform tasks well. }\end{array}$ \\
\hline 33 & S17 & They must also have values, personalities and diversity \\
\hline 34 & S17 & $\begin{array}{l}\text { The values, personalities and diversity must be appropriate to } \\
\text { the task }\end{array}$ \\
\hline 35 & S17 & $\begin{array}{l}\text { They must be sufficiently compatible for everyone to work } \\
\text { together. }\end{array}$ \\
\hline
\end{tabular}


$\underline{\text { Passage A10 }}$

\begin{tabular}{|c|c|c|}
\hline \# & $\begin{array}{l}\text { Sentence } \\
\#\end{array}$ & Idea Units \\
\hline 1 & S1 & $\begin{array}{l}\text { Resources and customers are two critical elements in the open- } \\
\text { systems view of organisations }\end{array}$ \\
\hline 2 & $\mathrm{~S} 2$ & $\begin{array}{l}\text { Resources must be put to good use for an organisation to perform } \\
\text { well. }\end{array}$ \\
\hline 3 & $\mathrm{~S} 2$ & $\begin{array}{l}\text { Customers must be well served for an organisation to perform } \\
\text { well. }\end{array}$ \\
\hline 4 & S3 & The notion of value creation is very important in this context. \\
\hline 5 & $\mathrm{~S} 4$ & $\begin{array}{l}\text { If operations add value to the original cost of the resource inputs, } \\
\text { then a business organisation can earn a profit. }\end{array}$ \\
\hline 6 & S4 & $\begin{array}{l}\text { A business can make a profit by selling a product for more than the } \\
\text { cost of making it. }\end{array}$ \\
\hline 7 & $\mathrm{~S} 4$ & Fast-food restaurant meals are an example of this. \\
\hline 8 & S5 & A not-for-profit organisation can add wealth to society. \\
\hline 9 & S5 & $\begin{array}{l}\text { They add value by providing a public service that is worth more } \\
\text { than its cost. }\end{array}$ \\
\hline 10 & S5 & Fire prevention services in a community are an example of this. \\
\hline 11 & S6 & $\begin{array}{l}\text { Value is created when an organisation's resources are used in the } \\
\text { right way. }\end{array}$ \\
\hline 12 & S6 & $\begin{array}{l}\text { Value is created when an organisation's resources are used at the } \\
\text { right time. }\end{array}$ \\
\hline 13 & S6 & $\begin{array}{l}\text { Value is created when an organisation's resources are used at } \\
\text { minimum cost to create high quality goods and services for } \\
\text { customers. }\end{array}$ \\
\hline 14 & S7 & The best organisations use a variety of performance measures. \\
\hline 15 & S8 & $\begin{array}{l}\text { On the customer side, high-performing organisations measure } \\
\text { customer satisfaction and loyalty, }\end{array}$ \\
\hline 16 & S8 & These organisations also measure market share. \\
\hline 17 & S9 & On the employee side, they measure retention. \\
\hline 18 & S9 & They measure career development. \\
\hline 19 & S9 & They measure job satisfaction. \\
\hline 20 & S9 & They measure related issues. \\
\hline 21 & S10 & $\begin{array}{l}\text { One of the most common indicators of organisational performance } \\
\text { overall is productivity. }\end{array}$ \\
\hline 22 & S10 & $\begin{array}{l}\text { Productivity is a summary measure of the quantity and quality of } \\
\text { work performance. }\end{array}$ \\
\hline 23 & S10 & Resource use is taken into account \\
\hline 24 & S11 & Productivity can be measured at the individual levels. \\
\hline 25 & S11 & Productivity can be measured at group levels \\
\hline 26 & S11 & Productivity can be measured at organisational levels \\
\hline 27 & S12 & Figure 1.2 links productivity with two terms. \\
\hline
\end{tabular}




\begin{tabular}{|c|c|c|}
\hline 28 & S12 & These terms are commonly used in management. \\
\hline 29 & S12 & These terms are effectiveness and efficiency. \\
\hline 30 & S13 & $\begin{array}{l}\text { Performance effectiveness is a measure of task output or goal } \\
\text { accomplishment }\end{array}$ \\
\hline 31 & S14 & $\begin{array}{l}\text { If you are working in the manufacturing area of a computer firm, } \\
\text { for example, performance effectiveness may mean that you meet a } \\
\text { daily production target. }\end{array}$ \\
\hline 32 & S14 & $\begin{array}{l}\text { Production targets are measured in terms of the quantity and } \\
\text { quality of keyboards assembled }\end{array}$ \\
\hline 33 & S15 & $\begin{array}{l}\text { By so doing, you allow the company as a whole to maintain its } \\
\text { production schedule }\end{array}$ \\
\hline 34 & S15 & $\begin{array}{l}\text { You also allow the company to meet customer demands for timely } \\
\text { delivery and high quality products. }\end{array}$ \\
\hline 35 & S16 & Productivity is the quantity and quality of work performance. \\
\hline 36 & S16 & Resource use is considered. \\
\hline 37 & S17 & $\begin{array}{l}\text { Performance effectiveness is an output measure of task or goal } \\
\text { accomplishment. }\end{array}$ \\
\hline
\end{tabular}


Passage A11

\begin{tabular}{|c|c|c|}
\hline \# & $\begin{array}{l}\text { Sentence } \\
\#\end{array}$ & Idea Unit \\
\hline 1 & S1 & System can be formally defined. \\
\hline 2 & S1 & A system is a collection of interrelated parts. \\
\hline 3 & S1 & $\begin{array}{l}\text { These interrelated parts in a system function together to achieve a } \\
\text { common purpose. }\end{array}$ \\
\hline 4 & $\mathrm{~S} 2$ & A subsystem is a smaller component of a larger system. \\
\hline 5 & S3 & $\begin{array}{l}\text { One of the earliest management writers to adopt a systems } \\
\text { perspective was Chester Barnard. }\end{array}$ \\
\hline 6 & S4 & Chester Barnard wrote a groundbreaking book. \\
\hline 7 & S4 & The book was written in 1938. \\
\hline 8 & S4 & The book was called Functions of the Executive. \\
\hline 9 & S4 & $\begin{array}{l}\text { The book was based on years of experience as a telephone } \\
\text { company executive. }\end{array}$ \\
\hline 10 & S5 & Barnard described organisations as cooperative systems. \\
\hline 11 & S5 & $\begin{array}{l}\text { Barnard said that the contributions of individuals in systems are } \\
\text { integrated for a common purpose. }\end{array}$ \\
\hline 12 & S6 & $\begin{array}{l}\text { Importantly, Barnard considered that this cooperation was } \\
\text { conscious. }\end{array}$ \\
\hline 13 & S6 & Barnard considered that this cooperation was deliberate. \\
\hline 14 & S6 & Barnard considered that this cooperation was purposeful. \\
\hline 15 & S7 & $\begin{array}{l}\text { It was the job of the executives, or managers, to make this } \\
\text { cooperation happen. }\end{array}$ \\
\hline 16 & S7 & They should do it through communication. \\
\hline 17 & S8 & $\begin{array}{l}\text { Systems thinking continues to influence management and practice } \\
\text { today. }\end{array}$ \\
\hline 18 & S9 & One application is described in Figure 4.4. \\
\hline 19 & S10 & This figure first depicts the larger organisation as an open system \\
\hline 20 & S10 & This system interacts with its environment. \\
\hline 21 & S10 & $\begin{array}{l}\text { The interaction with the environment entails the continual process } \\
\text { of transforming inputs from suppliers into outputs for customers. }\end{array}$ \\
\hline 22 & S11 & $\begin{array}{l}\text { Within the organisation any number of critical subsystems can be } \\
\text { described. }\end{array}$ \\
\hline 23 & S12 & $\begin{array}{l}\text { In the figure, the operations and service management systems are a } \\
\text { central point. }\end{array}$ \\
\hline 24 & S13 & They facilitate the interactions between other subsystems. \\
\hline 25 & S13 & $\begin{array}{l}\text { These interactions include activities such as purchasing, } \\
\text { accounting, sales and information }\end{array}$ \\
\hline 26 & S13 & These interactions are essential to the work of the organisation. \\
\hline 27 & S14 & $\begin{array}{l}\text { Importantly, high performance by the organisation as a whole } \\
\text { occurs only when each subsystem performs its task well. }\end{array}$ \\
\hline 28 & S14 & $\begin{array}{l}\text { High performance by the organisation as a whole occurs only } \\
\text { when each system works well in cooperation with the other }\end{array}$ \\
\hline
\end{tabular}




\begin{tabular}{|l|l|l|}
\hline & & subsystems. \\
\hline 29 & S14 & These ideas were suggested by Barnard. \\
\hline 30 & S15 & $\begin{array}{l}\text { It is the job of managers throughout the organisation to make this } \\
\text { coordinated action possible. }\end{array}$ \\
\hline 31 & S16 & $\begin{array}{l}\text { A system is a collection of interrelated parts working together for a } \\
\text { purpose. }\end{array}$ \\
\hline 32 & S17 & A subsystem is a smaller component of a larger system. \\
\hline 33 & S18 & Open systems transform resource inputs. \\
\hline 34 & S18 & They transform them from the environment into product outputs. \\
\hline
\end{tabular}




\section{REFERENCES}

Abisamra, N.S. (2001). Teaching second language reading from an interactive perspective. Retrieved from http://nadabs11.tripod.com/reading/ .

Abu-Rabia, S. (1996). Druze minority students learning Hebrew in Israel: The relationship of attitudes, cultural background, and interest of material to reading comprehension in a second language. Journal of Multilingual and Multicultural Development, 17(6), 415-426.

American College Testing. (2006, 1 March). Reading between the lines: What the ACT reading test reveals about college readiness. Retrieved from http://www.act.org/path/policy'reports/reading.html

Aebersold, J.A. \& Field, M.L. (1997). From reading to reading teacher. Issues and strategies for second language classrooms. Cambridge, England: Cambridge University Press.

Afflerbach, P. (2000). Verbal reports and protocol analysis. In M.L. Kamil, P.B. Mosenthal, P.D. Pearson \& R. Barr (Eds.), Handbook of Reading Research. Volume III (pp. 163-180). Mahwah, NJ: Lawrence Erlbaum Associates.

Airini, D.B., Curtis, E., Johnson, O., Luatua, F., O’Shea, M., Rakena, T., Reynolds, G., Sauni, P., Smith, A., Huirua, T.S., Tarawa, M., UlugiaPua, M. (2009). Success for all: Improving Maori and Pasifika student success in degree-level studies. Milestone report 8. Auckland, New Zealand: The University of Auckland.

Ajideh, P. (2003). Schema Theory-based pre-reading tasks: A neglected essential in the ESL reading classroom. The Reading Matrix, 3(1). Retrieved from http://www.readingmatrix.com/articles/ajideh/article.pdf.

Alexander, P.A, Jetton, T.L. \& Kulikowich, J.M. (1995). Interrelationship of knowledge, interest, and recall: Assessing a model of domain learning. Journal of Educational Psychology, 87(4), 559-575.

Alptekin, C. (2006). Cultural familiarity in inferential and literal comprehension in in L2 reading. System, 34, 494-508.

Anae, M., Coxon, E., Mara, D., Wendt-Samu, T. \& Finau, C. (2001). Pasifika education research guidelines. Wellington, New Zealand: Ministry of Education.

Anderson, N.J. (1991). Individual differences in strategy use in second language reading and testing. The Modern Language Journal, 75(4), pp.460-472.

Armbruster, B.B. \& Anderson, T.H. (1990). The effect of mapping on the free recall of expository text. Technical report No.160, Centre for the Study 
of Reading, University of Illinois at Urbana-Champaign. Retrieved from https://test.ideals.illinois.edu/bitstream/handle/123456789/7935/ctrstread techrepv01980i00160_opt.pdf? sequence $=1$.

Armstrong, S.L. (2008). Using metaphor analysis to uncover learners' conceptualizations of academic literacies in postsecondary developmental contexts. The International Journal of Learning, 15(9), 211-218.

Auerbach, E.R. \& Paxton, D. (1997). "It's not the English thing": Bringing reading research into the ESL classroom. TESOL Quarterly, 31(2), 237255.

Bailin, A. \& Grafstein, A. (2001). The linguistic assumptions underlying readability formulae: A critique. Language and Communication, 21(3), 285-301.

Baker, L. \& Brown, A.L. (2002). Metacognitive skills and reading. In P.D. Pearson (Ed.), Handbook of Reading Research (pp.353-394). Mahwah, NJ: Lawrence Erlbaum.

Baldwin, R.S., Peleg-Bruckner, Z. \& McClintock, A.H. (1985). Effects of topic interest and prior knowledge on reading comprehension. Reading Research Quarterly, 20(4), 497-504.

Bang, H.J. \& Zhao, C.G. (2007). Reading strategies used by advanced Korean and Chinese ESL graduate students: A case study. The Reading Matrix, 7(1), 30-50.

Barnett, M. (1986). Syntactic and lexical/semantic skill in foreign language reading: Importance and interaction. Modern Language Journal, 70, 343-49.

Barton, D. \& Hamilton, M. (1998). Local literacies: Reading and writing in one community. London: Routledge.

Basham, C., Ray, R. \& Whalley, E. (1993). Cross-Cultural perspectives on task representation in reading to write. In J.G. Carson \& I. Leki (Eds.), Reading in the compositions classroom: Second language perspectives. Boston, MA: Heinle and Heinle.

Baynam, M. (1995). Literary practices: Investigating literacy in social contexts. London, England: Longman.

Bean, T.W. (2000). Reading in the content areas: Social constructivist dimensions. In M.L. Kamil, P.B. Mosenthal, P.D. Pearson \& R. Barr (Eds.), Handbook of Reading Research. Volume III (pp. 629-644). Mahwah, NJ: Lawrence Erlbaum Associates.

Beers, T. (1987). Schema-theoretic models of reading: Humanizing the machine. Reading Research Quarterly, 22(3), 369-377.

Bell, J. (2007). Reading practices: Postgraduate Thai student perceptions. The Reading Matrix, 7(1), 51-68. 
Belloni, L.F. \& Jongsma, E.A. (1978). The effects of interest on reading comprehension of low achieving students. Journal of Reading, 22(2), 106-109.

Benseman, J., Sutton, A. \& Lander, J. (2003). Foundation learning in Aotearoa New Zealand. Retrieved from

http://www.educationcounts.govt.nz/publications/tertiary_education/277 73/28033/28029.

Behrman, E.H. (2004). "The Culture of Reading" in a public school. Ethnography, Service-Learning, and Undergraduate Researchers. Retrieved from http://130.91.80.97:591/PDFs/46-3/The\%20Culture.pdf.

Behrman, E.H. \& Street, C. (2005). The validity of using a content-specific reading comprehension test for college placement. Journal of College Reading and Learning, 35(2), 5-22.

Berkowitz, S.J. (1986). Effects of instruction in text organization on sixthgrade students' memory for expository reading. Reading Research Quarterly, 21(2), 161-178.

Berman, R. (1984). Syntactic components of the FL reading process. In J.C. Alderson \& A.H. Urquhart (Eds.), Reading in a Foreign Language. Longman.

Bernhardt, S.A. (1986). Seeing the text. College Composition and Communication, 37(1), 66-78.

Bernhardt, E.B. (1991). Reading Development in a Second Language: Theoretical, empirical \& classroom perspectives. Norwood, NJ: Ablex.

Bernhardt, E.B. (2000). Second language reading as a case study of reading scholarship in the $20^{\text {th }}$ century. In M.L. Kamil, P.B. Mosenthal, P.D. Pearson \& R. Barr (Eds.), Handbook of Reading Research. Volume III (pp. 791-812). Mahwah, NJ: Lawrence Erlbaum Associates.

Besser, S., Brooke, G., Burton, M., Parisella, M., Spare, Y., Stratford, S. \& Wainwright, J. (2004). Adult literacy learners' difficulties in reading: an exploratory study. A Research Report for the National Research and Development Centre for adult literacy and numeracy. London: National Research and Development Centre. Retrieved from www.nrdc.org.uk.

Birch, B. (2007). English L2 Reading: Getting to the Bottom (2nd Ed.) Mahwah, NJ: Lawrence Erlbaum Associates.

Block, E. (1986). The comprehension strategies of second language readers. TESOL Quarterly, 20(3), 463-494.

Block, E. (1992). See how they read: Comprehension monitoring of L1 and L2 Readers. TESOL Quarterly, 26(2), 319-343.

Bouvet, E. (2002). Reading in a foreign language: strategic variation between readers of differing proficiency. Flinders University Language Group 
Online Review, 1(1). Retrieved from http://ehlt.flinders.edu.au/deptlang/fulgor/.

Brown, A.L. (1980). Metacognition development and reading. In R.J. Spiro, B.C. Bruce \& W.F. Brewer (Eds.), Theoretical issues in reading comprehension (pp.453-481). Hillsdale, NJ: Erlbaum Associates.

Brown, A.L. \& Campione, J.C. (1996). Communities of learning and thinking, or a context by any other name. In P.Woods (Ed.), Contemporary Issues in Teaching and Learning (pp.120-126). London, England: Routledge

Brown, C.L. (2007). Supporting English language learners in content-reading. Reading Improvement, 44(1), 32-39.

Brown, C., Snodgrass, T., Kemper, S.J., Herman, R. \& Covington, M.A. (2008). Automatic Measurement of Propositional Idea Density from Part-of-Speech Tagging. Retrieved from http://www.pubmedcentral.nih.gov/articlerender.fcgi?artid=2423207.

Camiciottoli, B.C. (2003). Metadiscourse and ESP Reading Comprehension: An Exploratory Study. Reading in a Foreign Language, 15(1), 28-44.

Caplan, N. \& Nelson, S. D. (1973). On being useful: The nature and consequences of psychological research on social problems. American Psychologist, 28, 199-211.

Carrell, P.L. (1985). Facilitating ESL Reading by Teaching Text Structure. TESOL Quarterly, 19(4), 727-752.

Carrell, P.L. \& Carson, J.G. (1997). Extensive and intensive reading in an EAP setting. ESP Journal, 16(1), 47-60.

Carrell, P. L. \& Eisterhold, J. C. (1983). Schema theory and ESL reading. TESOL Quarterly, 17, 218-232.

Carrell, P. C. (1985). Facilitating ESL reading by teaching text structure. TESOL Quarterly, 19, 727-752.

Carrell, P.L. (1987). Content and formal schemata in ESL reading. TESOL Quarterly, 21(3), 461-481.

Carter-Wells, J. (1996). Raising expectations for critical reading. New Directions For Higher Education, 96, 45-54.

Carver, R. P. (1990). Reading rate: A review of research and theory. San Diego, CA: Academic Press.

Carver, R.P. (1994). Percentage of unknown vocabulary words in text as a function of the relative difficulty of the text: Implications for instruction. Journal of Reading Behavior, 26, 413-437.

Cassanave, C.P. (1988). Comprehension monitoring in ESL reading: A neglected essential. TESOL Quarterly, 22(2), 283-302.

Cervetti, G., Pardales, M.J., \& Damico, J.S. (2001). A tale of differences: Comparing the traditions, perspectives, and educational goals of critical 
reading and critical literacy. Reading Online. Retrieved from http://www.readingonline.org/articles/cervetti/index.html.

Chamot, A.U. \& O'Malley, J.M. (1994). The CALLA handbook: Implementing the Cognitive Academic Language Learning Approach. White Plains, NY: Addison, Wesley, Longman.

Chandler, D. (n.d.). An introduction to genre theory. Retrieved from http://www.aber.ac.uk/media/Documents/intgenre/intgenre4.html.

Chang, Y-F. (2006). On the use of the immediate recall task as a measure of second language reading comprehension. Language Testing, 23(4), 520543.

Chaparro, B.S., Shaikh, A.D. \& Baker, J.R. (2005). Reading online text with a poor layout: Is performance worse? Usability News, 7(1). Retrieved from http://www.surl.org/usabilitynews/71/page_setting.asp .

Chi, F-M. (1995). EFL readers and a focus on intertextuality. Journal of Reading, 38(8), 638-644.

Clarke, M.A. (1980). The short circuit hypothesis of ESL reading - Or when language competence interferes with reading performance. The Modern Language Journal, 64(2), 203-209.

Coady, J. (1997). L2 vocabulary acquisition through extensive reading. In J. Coady \& T. Huckin (Eds.), Second Language Vocabulary Acquisition (pp. 225-237). Cambridge, England: Cambridge University Press.

Cobb, T. (n.d.). Web Vocabprofile. [an adaptation of Heatley \& Nation's (1994) Range. Retrieved 2009 from http://www.lextutor.ca/vp/ ].

Cobb, T. \& Horst, M. (2001).Reading academic English: Carrying learners across the lexical threshold. In J. Flowerdew \& M. Peacock (Eds.), Research perspectives on English for academic purposes (pp. 315-330). Cambridge, England: Cambridge University Press.

Cohen, A.D. \& Horowitz, R. (2002). What should teachers know about bilingual learners and the reading process? In J.H. Sullivan (Ed.), Literacy and the Second Language Learner (pp. 29-53). Volume 1 of Research in Second Language Learning. Greenwich, CT: Information Age Publishing.

Comparative Education Research Unit. Ministry of Education. (2004). Focus on Pasifika Students Achievement in reading Literacy: Results from PISA 2000. Wellington, New Zealand: Ministry of Education. Retrieved from

http://www.educationcounts.govt.nz/publications/series/2543/pisa_2000/ focus_pasifika_2000.

Conley, D.T. (2008). Rethinking College Readiness. New Directions for Higher Education, 144, 3-13. 
Coulthard, M. (2001). On analyzing and evaluating written text. In M. Coulthard (Ed.), Advances in written text analysis (pp. 1-11). London, England: Routledge.

Coxhead, A. (2000). A New Academic Word List. TESOL Quarterly, 34(2), 213-238.

Cummins, J. (1979). Linguistic interdependence and the educational development of bilingual children. Review of Educational Research, 49(2), 222-251.

Davidson-Toumu'a, M.R. (2005). The tertiary academic reading experience: Case studies of first year Pacific students (Unpublished master's thesis). Victoria University of Wellington, Wellington, New Zealand.

Davis, K.A. (1995). Qualitative Theory and Methods in Applied Linguistics Research. TESOL Quarterly: Qualitative Research in ESOL, 29(3), 427453.

Dennis, S. \& Kintsch, W. (n.d.). The text mapping and inference rule generation problems in text generation: Evaluating a memory-based account. Retrieved from http://209.85.173.104/custom?q=cache:rFf4JbSYr2YJ:mall.psy.ohiostate.edu/TextMapping.pdf+\%22text-

mapping $\% 22 \& \mathrm{hl}=\mathrm{en} \& \mathrm{ct}=\mathrm{clnk} \& \mathrm{~cd}=8 \& \mathrm{gl}=\mathrm{nz} \& \mathrm{client}=\mathrm{pub}-$ 9081652265667472 .

Devine, J. (1988). The relationship between general language competence and second language reading proficiency: Implications for teaching. In P.L. Carrell, J. Devine \& D.E. Eskey (Eds.), Interactive approaches to second language reading (pp.260-278). Cambridge, England: Cambridge University Press.

Devine, J. (1993). The role of metacognition in second language reading and writing. In J.G. Carson \& I. Leki (Eds.), Reading in the composition classroom: Second language perspectives (pp.105). Boston, MA: Heinle and Heinle.

Dickie, J. (n.d.). Pasifika students in New Zealand schools: Some explanations for their literacy performance. Retrieved from http://www.aare.edu.au/03pap/dic03191.pdf .

Dreher, M.J., \& Singer, H. (2001). Friendly texts and text-friendly teachers. Theory Into Practice, XXVIII(2), 98-104.

Dreyer, C. \& Nel, C. (2003). Teaching reading strategies and reading comprehension within a technology-enhanced learning environment. System, 31, 349-365.

Dudley-Evans, T. (n.d.). Genre analysis: a key to a theory of ESP? Retrieved from http://www.aelfe.org.documents/text2-Dudley.pdf. 
Duke, N.K. \& Pearson, P.D. (2002). Effective Practices for Developing Reading Comprehension. In A.E. Farstrup \& S.J. Samuels (Eds.), What research has to say about reading instruction (pp. 205-242). Newark, DE: International Reading Association.

Dunworth, K. (2008). A Task-Based Analysis of Undergraduate Assessments: A Tool for the EAP practitioner. TESOL Quarterly, 42, 315-323.

Edwards, P.A. \& Turner, J.D. (2009). Family literacy and reading comprehension. In G.G. Duffy \& S. Israel (Eds.), Handbook of Research on Reading Comprehension. NY: Routledge.

Eskey, D.E. (1976, July). Toward a theory of second language reading. A paper presented to the Conference on Second Language Learning and Teaching, Oswego, NY. Retrieved from http://eric.ed.gov/PDFS/ED132845.pdf.

Fanene, N. (2006). A study of the academic writing problems of New Zealandborn Samoan students in tertiary institutions. (Master's thesis, Auckland University of Technology, Auckland, New Zealand). Retrieved from http://repositoryaut.lconz.ac.nz/handle/10292/271.

Field, M.L. \& Aebersold, J. (1990). Cultural attitudes toward reading: Implications for teachers of ESL/bilingual readers. Journal of Reading, 33(6), 406-410.

Fiocco, M. (1996). Tertiary literacy: Definition and practice within one university. What is the relationship to language theory? HERDSA Conference Proceedings. Retrieved from http://www.herdsa.org.au/confs/1996/fiocco.html.

Flockton L. \& Crooks, T. (2001). Reading and speaking. Assessment results 2000. National education monitoring report 19. Retrieved from http://nemp.otago.ac.nz/read_speak/2000/index.htm.

Florio-Ruane, S. \& McVee, M. (2000). Ethnographic approaches to literacy research. In M. L. Kamil, P. B. Mosenthal, P. D. Pearson, \& R. Barr (Eds.), Handbook of Reading Research (pp. 153-162). Mahwah, NJ: Lawrence Erlbaum.

Flower, L., Stein, V., Ackerman, J., Kantz, M.J., McCormick, K. \& Peck, W.C. (1990). Reading to write: Exploring a cognitive and social process. New York, NY: Oxford University Press.

Floyd, P. \& Carrell, P. (1987). Effects on ESL reading of teaching cultural content schemata. Language Learning, 37, 89-108.

Francis, H. \& Hallam, S. (2000). Genre effects on higher education students' text reading for understanding. Higher Education, 39, 279-296.

Fraser, C.A. (1989). Reading for Independence. TESL Canada Journal, 6(2), 74-86. 
Fredericksen, C.H. (1975). Acquisition of semantic information for discourse: Effects of repeated exposure. Journal of Verbal Learning and Verbal Behavior, 14, 158-169.

Fulcher, G. (1997). Text difficulty and accessibility: Reading formulae and expert judgement. System, 25(4), 497-513.

Garner, R. (1981). Monitoring of passage inconsistency among poor comprehenders: A preliminary test of the "piecemeal processing" explanation. Journal of Educational Research, 74, 159-162.

Garner, R., Alexander, P.A., Gillingham, M.G., Kulikowich, J.M. \& Brown, R. (1991). Interest and learning from text. American Educational Research Journal, 28, 643-659.

Gebel, T.A. \& Schrier, L.L. (2002). Spanish language teachers' beliefs about reading in a second language. In J.H.Sullivan (Ed.), Literacy and the Second Language Learner (pp. 85-109). Volume 1 of Research in Second Language Learning. Greenwich, CT: Information Age Publishing.

Gibbons, P. (2002). Scaffolding language learning: Teaching second language learners in the mainstream classroom. Portsmouth, NH: Heinemann.

Goodman, K. (1982). Language and literacy. Boston, MA: Routledge \& Keegan.

Grabe, W. (1991). Current developments in second language reading research. TESOL Quarterly, 25(3), 375-406.

Grabe, W. (2010). Revisiting the MLA Report on reconfiguring foreign language programs. Reading in a Foreign Language, 22(Supplement1), 11-14.

Green, A. (2006). University challenge, dynamic subject knowledge, teaching and transition. Arts and Humanities in Higher Education, 5, 275-290.

Grimes, S.K. \& David, K.C. (1999). Underprepared community college students: Implications of attitudinal and experiential differences.

Community College Review. Retrieved from http://findarticles.com/p/articles/mi_m0HCZ/is_2_27/ai_63323069.

$\mathrm{Gu}$, P.Y. (2003). Vocabulary learning in a second language: Person, task, context and strategies. TESL-EJ, 7(2). Retrieved from http://www.teslej.org/wordpress/issues/volume7/ej26/ej26a4/.

Guthrie, J.T., Coddington, C.S. \& Wigfield, A. (2009). Profiles of reading motivation among African American and Caucasian students. Journal of Literacy Research, 41(3), 317-353.

Health Research Council. (2004). Guidelines for Pacific health research. Wellington, New Zealand: Health Research Council of New Zealand.

Heatley, A. and Nation, P. (1994). Range. Victoria University of Wellington, NZ. [Computer program, available at http://www.vuw.ac.nz/lals/]. 
Helu-Thaman, K. (2009). Towards cultural democracy in teaching and learning with specific references to Pacific Island Nations (PINs). International Journal for the Scholarship of Teaching and Learning, 3(2). Retrieved from http://academics.georgiasouthern.edu/ijsotl/v3n2/invited_essays/PDFs/In vitedEssay_Thaman.pdf.

Hermida, J. (2009). The Importance of Teaching Academic Reading Skills in First-Year University Courses. Retrieved from SSRN: http://ssrn.com/abstract=1419247.

Hidi, S. (2001). Interest, Reading and Learning: Theoretical and Practical Considerations. Educational Psychology Review, 13(3), 191-209.

Hirst, E., Henderson, R., Allan, M., Bode, J. \& Kocatepe, M. (2004). Repositioning academic literacy: Charting the emergence of a community of practice. Australian Journal of Language and Literacy, 27(1), 66-80.

Hittleman, D.R. (1978). Readability, readability formulas, and cloze: Selecting instructional materials. Journal of Reading, 22(2), 117-122.

Horiba, Y. (2000). Reader Control in reading: Effects of language competence, text type, and task. Discourse Processes, 29(3), 223-267.

$\mathrm{Hu}$, M. \& Nation, P. (2000). Unknown vocabulary density and reading comprehension. Reading in a Foreign Language, 13(1), 403-430.

Huang, S. (2006). Reading English for academic purposes - What situational factors may motivate learners to read? System, 34, 371-383.

ICAS. (2002). Academic Literacy: A Statement of Competencies Expected of Students Entering California's Public Colleges and Universities. Sacramento, CA: Intersegmental Committee of the Academic Senates (ICAS). Retrieved from http://www.universityofcalifornia.edu/senate/reports/acadlit.pdf.

Jackson, N. E. (2005). Are university students' component reading skills related to their text comprehension and academic achievement? Learning and Individual Differences, 15, 113-139.

Johns, A.M. (1995). Teaching classroom and authentic genres: Initiating students into academic cultures and discourses. In D. Belcher \& G. Braine (Eds.), Academic Writing in a Second Language: Essays on Research and Pedagogy (pp. 277-291). Norwood, NJ: Ablex Publishing.

Johnson, D.M., \& Saville-Troike, M. (1992). Validity and reliability in qualitative research on second language acquisition and teaching. TESOL Quarterly, 26(3), 602-605.

Juel, C. (1988). Learning to Read and Write: A Longitudinal Study of FiftyFour Children from First Through Fourth Grade. Journal of Educational Psychology, 80, 437-447. 
Just, M.A. \& Carpenter, P.A. (1980). A theory of reading: From eye fixations to comprehension. Psychological Review, 87(4), 329-354.

Kelder, R. (1998). Literacy, the Self, and Culture: An Enquiry. Information Analyses. Retrieved from http://eric.ed.gov/ERICDocs/data/ericdocs2sq1/content_storage_01/0000 019b/80/17/39/b3.pdf .

Kemp, C.G. (1963). Improvement of critical thinking in relation to openclosed belief systems. Journal of Experimental Education, 31(3), 321323.

Kintsch, W. \& Keenan, J. (1973). Reading rate and retention as a function of the number of propositions in the base structure of sentences. Cognitive Psychology, 5, 257-274.

Koda, K. (2005). Insights into second language reading: A cross-linguistic approach. Cambridge, England: Cambridge University Press.

Kong, A. (2006). Connections between L1 and L2 readings; Reading strategies used by four Chinese adult readers. The Reading Matrix, 6(22), 19-45.

Koo, Y.L. \& Soo Hoo, P.L. (2007). The Social construction of literacy by Malaysian Chinese parents: Perceptions of parents toward the language and literacy practices of two teenage children. The Reading Matrix, 7(3), 72-87.

Kovach, M. (2009). Indigenous methodologies: Characteristics, conversations, and contexts. Toronto, Canada: University of Toronto Press.

Krieger, E. (1990). Developing comprehension through author awareness. Journal of Reading, 33(8), 618-619.

LaBerge, D. \& Samuels, S.J. (1974). Toward a theory of automatic information processing in reading. Cognitive Psychology, 6(2), 293323.

Larsen-Freeman, D. \& Long, M.H. (1991). An introduction to second language research. London, England: Longman.

Lau, K. (2006). Reading strategy use between Chinese good and poor readers: a think-aloud study. Journal of Research in Reading, 29(4), 383-399.

Lau, K. \& Chan, D.W. (2003). Reading strategy use and motivation among Chinese good and poor readers in Hong Kong. Journal of Research in Reading, 26(2), 177-190.

Laufer, B. (1989). What percentage of text-lexis is essential for comprehension? In C. Lauren \& M. Nordman (Eds.), Special language: From humans thinking to thinking machines (pp. 316-323). Avon, England: Multilingual Matters. 
Laufer, B. (1997). The lexical plight in second language reading: Words you don't know, words you think you know, and words you can't guess. In J. Coady \& T. Huckin (Eds.), Second language vocabulary acquisition (pp. 20-34). Cambridge, England: Cambridge University Press.

Laufer, B. \& Nation, I. S. P. (1995). Vocabulary size and use: Lexical richness in L2 written production. Applied Linguistics, 16, 307-322.

Lee, J. \& Schallert, D.L. (1997). The relative contribution of L2 language proficiency and L1 reading ability to L2 reading performance: A test of the threshold hypothesis in an EFL context. TESOL Quarterly, 31(4), 713-739.

Lee, S-K. (2009). Topic congruence and topic interest: How do they affect second language reading comprehension? Reading in a foreign language, 21(2), 159-178.

Lewandowski, L.J., Codding, R.S., Kleinmann, A.E. \& Tucker, K.L. (2003). Assessment of reading rate in postsecondary students. Journal of Psychoeducational Assessment, 21, 134-144.

Lin, H. \& Chen, T. (2006). Decreasing cognitive load for novice EFL learners: Effects of question and descriptive advance organizers in facilitating EFL learners' comprehension of an animation-based content lesson. System, 34, 416-431.

Lincoln, Y.S. \& Guba, E.G. (1985). Naturalistic Inquiry. Newbury Park, CA: Sage

Livingstone, S.M. (1993). The rise and fall of audience research: An old story with a new ending. Journal of Communication, 43(4), 5-12.

Lonon-Blanton, L. (1993). Reading as performance: Reframing the function of reading. In J.G. Carson \& I. Leki (Eds.), Reading in the Compositions Classroom: Second Language Perspectives. MA: Heinle and Heinle.

Lorch, R.F. \& Lorch, E.P. (1995). Effects of organizational signals on textprocessing strategies. Journal of Educational Psychology, 87(4), 537544.

Martino, N.L. \& Hoffman, P.R. (2002). An investigation of reading and language abilities of college freshmen. Journal of Research in Reading, 25(3), 310-318.

Mangubhai, F. (1995). Invitation to another world: Literacy in the Pacific. Directions: Journal of Educational Studies, 17(1), 3-20.

Mann, S.J. (2000). The student's experience of reading. Higher Education, 39(3), 297-317.

Manzo, A.V., Manzo, U., Barnhill, A. \& Thomas, M. (2000). Proficient reader subtypes: Implications for literacy theory, assessment, and practice. Reading Psychology, 21, 217-232. 
Marshall, S. \& Gilmour, M. (1993). Lexical knowledge and reading comprehension in Papua New Guinea. English for Specific Purposes, 12(1), 69-81.

Masson, M.E.J. \& Sala, L.S. (1978). Interactive processes in sentence comprehension and recognition. Cognitive Psychology, 10(2), 244-270.

Matthews, M. (1989). Woods and trees: The correction of myopia in L2 reading. Reading in a Foreign Language, 6(1), 357-361.

McInnis, C. \& James, R. (1995). First year on campus: Diversity in the initial experiences of Australian undergraduates. Canberra, Australia: Australian Government Publishing Service. Retrieved from http://ultibase.rmit.edu.au/Articles/june97/three1.htm.

McHardy, J. (2009). The characteristics of adult readers in entry level tertiary settings in New Zealand. A thesis presented to the University of Waikato, New Zealand, in fulfillment of the requirement for the Degree of Master of Education. Retrieved from the University of Waikato Research Commons http://hdl.handle.net/10289/3588.

Meyer, B.J.F., Brandt, D.M. \& Bluth, G.J. (1980). Use of top-level structure in text: Key comprehension of ninth-grade students. Reading Research Quarterly, 16(1), 72-103.

Meyer, B.J.F. (1975). The organization of prose and its effect on memory. Amsterdam, Netherlands: North Holland.

Meyer, B. J., \& McConkie, G. W. (1973). What is recalled after hearing a passage? Journal of Educational Psychology, 65, 109-117.

Meyer, B.J.F., Talbot, A.P., \& Florencio, D. (1999). Reading Rate and Prose Retrieval. Scientific Studies of Reading, 3(4), 303-329.

Meyer, B.J.F. (2003). Text coherence and readability. Topics in Language Disorder, 23(3), 204-224.

Miles, M.B. (1994). Qualitative Data Analysis. Thousand Oaks, CA: Sage Publications.

Ministry of Education. (n.d.). Focus on Pasifika Achievement in Reading Literacy. Results from PISA 2000. Wellington, New Zealand: Ministry of Education. Retrieved from http://educationcounts.edcentre.govt.nz/publications/pasifika/literacypisa2000.html.

Ministry of Education. (2005). Tertiary Education Learning Outcomes Policy, Ministry of Education. Wellington, New Zealand: Ministry of Education. Retrieved from http://www.educationcounts.govt.nz/publications/tertiary_education/277 73/5495. 
Mokhtari, K. \& Reichard, CA. (2002). Assessing students' metacognitive awareness of reading strategies. Journal of Educational Psychology, 94(2), 249-259.

Moore, T. \& Morton, J. (2005). Dimensions of difference: A comparison of university writing and IELTS writing. Journal of English for Academic Purposes, 4(1), 43-66.

Nagy, W. E., \& Scott, J. A. (2000). Vocabulary processes. In M. L. Kamil, P. B. Mosenthal, P. D. Pearson, \& R. Barr (Eds.), Handbook of reading research, Vol. III (pp. 269-284). Mahwah, NJ: Erlbaum.

Nation, I.S.P. \& Beglar, D. (2007). A vocabulary size test. The Language Teacher, 31(7), 9-13.

Nation, I.S.P. (2007). Vocabulary Size Test, BNC Version (1-14K). [print version of online test]. Retrieved from http://www.lextutor.ca/tests/.

Nelson, G. (1993). Reading and writing: Integrating cognitive and social dimensions. In J.G. Carson, \& I. Leki (Eds.), Reading in the compositions classroom: Second language perspectives. MA: Heinle and Heinle.

Neuman, S.B. \& Celano, D. (2001). Access to print in middle- and lowincome communities: An ecological study of four neighborhoods. Reading Research Quarterly, 36, 8-26.

New Zealand Qualifications Authority (NZQA). (2010). Annual Report on NCEA \& New Zealand Scholarship data and statistics 2009. Wellington, New Zealand: New Zealand Qualifications Authority.

Nilgupta, S. (1978). The relationship of syntax to readability for ESL students in Thailand. In D. Feitelson (Ed.), Cross-Cultural Perspectives on Reading and Reading Research (pp. 89-102). Newark, DE: International Reading Association.

Nist, S.L., \& Holschuh, J.P. (2005). Practical applications of the research on epistemological beliefs. Journal of College Reading and Learning, 35(2), 84-93.

Nist, S.L. \& Simpson, M. (2000). College Studying. Retrieved from http://www.readingonline.org/articles/handbook/nist/.

Nuttall, C. (1982). Teaching Reading Skills in a Foreign Language. London, England: Heinemann Educational Books.

Obah, T. (1982). Reading in higher education in Nigeria: Problems and progress. Journal of Reading, 25(4), 315-321.

O'Leary, Z. (2004). The essential guide to doing research. London, England: Sage Publications.

Oxford, R.L. (1997). Cooperative Learning, Collaborative Learning, and Interaction: Three communicative strands in the language classroom. The Modern Language Journal, 81(4), 443-456. 
Pani, S. (2004). Reading strategy instruction through mental modeling. ELT Journal, 58(4), 355-362.

Paris, S.G. (2005). Reinterpreting the development of reading skills. Reading Research Quarterly, 40(2), 184-202.

Paris, S.G., Wasik, B.A. \& Turner, J.C. (1991). The development of strategic readers. In R. Barr, M. Kamil, P. Mosenthal, \& P.D. Pearson (Eds.), Handbook of reading research (pp.609-640). NY: Longman.

Parry, K. (1996). Culture, literacy, and L2 reading. TESOL Quarterly, 30(4), 665-692.

Pearson, P.D. (2009). The roots of reading comprehension instruction. In S.E. Israel, \& G.G. Duffy (Eds.), Handbook of Research on Reading Comprehension (pp. 3-31). NY: Routledge.

Peña Calvo, A. (2004). Reading strategically. A study of the teaching of reading strategies to university students. CILT Research ForumStrategies in Language Learning. Retrieved from http://www.cilt.org.uk/research/resfor1/calvo.htm.

Perfetti, C.A. (1969). Lexical density and phrase structure depth as variables in sentence retention. Journal of Verbal Learning and Verbal Behavior, $8(6), 719-724$.

Pinker, S. (1989). Learnability and cognition: The acquisition of argument structure. Cambridge MA: MIT Press.

Pithers, R.T. \& Soden, R. (2000). Critical thinking in education: a review. Educational Research, 42(3), 237-249.

Portnuff Venable, G. (2003). Readability case studies and scenarios. Topics in Language Disorders, 23(3), 204-221.

Posner, M.I. \& Snyder, C.R.R. (1975). Attention and cognitive control. In R.L. Solso, (Ed), Information Processing and Cognition: The Loyola symposium. Lawrence Erlbaum.

Prater, K. (2009). Reading comprehension and English language learners. In S.E. Israel \& G. G. Duffy (Eds.), Handbook of Research on Reading Comprehension (pp. 607-621). NY: Taylor \& Francis.

Pretorius, E.J. (2000). "What they can't read will hurt them": Reading and academic achievement. Innovation, 21, 33-41. Retrieved from http://www.innovation.ukzn.ac.za/InnovationPdfs/No21pp3341Pretorius.pdf.

Pretorius, E.J. (2006). The comprehension of logical relations in expository texts by students who study through the medium of ESL. System, 34, 432-450.

Pucci, S. (1994). Supporting Spanish language literacy: Latino children and free reading resources in schools. Bilingual Research Journal, 18, 6782. 
Railton, D. \& Watson, P. (2005). Teaching autonomy: 'Reading groups' and the development of autonomous learning practices. Active Learning in Higher Education, 6,182-193.

Rapp, D.N. \& van den Broek, P. (2005). Dynamic text comprehension: An integrative view of reading. Current Directions in Psychological Science, 14(5), 276-279.

Ridgeway, 2003

Rubens, P.M. (1986). A reader's view of text and graphics: Implications for transactional text. Journal of Technical Writing and Communication, 16(1-2), 73-86.

Rumelhart, D.E. \& Ortony, A. (1977). In R.C. Anderson, R.J. Spiro \& W.E. Montague (Eds.), Schooling and the acquisition of knowledge. Hillsdale, N.J.: Lawrence Erlbaum.

Sadoski, M., Goetz, E.T. \& Fritz, J.B. (1993). Impact of concreteness on comprehensibility, interest and memory for text: Implications for dual coding theory and text design. Journal of Educational Psychology, 85(2), 291-304.

Salataci, R. \& Akyel, A. (2002). Possible Effects of Strategy Instruction on L1 and L2 Reading. Reading in a Foreign Language, 14(1), 1-17.

Säljö, R. (1982). Learning and understanding: A study of differences in constructing meaning from a text. Göteborg, Sweden: Acta Universitatis Gothoburgensis.

Scarcella, R.C. \& Oxford, R.L. (1992). The tapestry of language learning: The individual in the communicative classroom. TESL-EJ, 1(3). Retreived from http://www.tesl-ej.org/wordpress/issues/volume1/ej03/ej03r20/.

Schmitt, N, Schmitt, D. \& Clapham, C. (2001). Developing and exploring the behavior of two new versions of the Vocabulary Levels Test. Language Testing, 18(1), 55-88.

Schneider, B. (2001). Fits about fit. Applied Psychology: An International Review, 50(1), 141-152.

Schraw, G. \& Bruning, R. (1996). Readers' implicit models of reading. Reading Research Quarterly, 31(3), 290-305.

Schumm, J.S. \& Mangrum II, C.T. (1991). FLIP: A framework for content area reading. Journal of Reading, 35(2), 120-127.

Scott, D. \& Smart, W. (2005). What factors make a difference to getting a degree in New Zealand? Wellington, New Zealand: Tertiary Sector Performance Analysis and Reporting, Ministry of Education.

Scribner, S. \& Cole, M. (1981). The psychology of literacy. Harvard University Press.

Sengupta, S. (2002). Developing academic reading at tertiary level: A longitudinal study tracing conceptual change. The Reading Matrix, 2(1). 
Retrieved from

http://www.readingmatrix.com/articles/sengupta/index.html.

Shearer, B.A., Lundeberg, M.A. \& Coballes-Vega, C. (1997). Making the connection between research and reality: Strategies teachers use to read and evaluate journal articles. Journal of Educational Psychology, 89(4), 593-598.

Sheorey, R. \& Mokhtari, K. (2001). Differences in the metacogntive awareness of reading strategies among native and non-native readers. System, 29, 431-449.

Singhal, M. (2001). Reading Proficiency, Reading Strategies, Metacognitive Awareness and L2 Readers. The Reading Matrix, 1(1). Retrieved from http://www.readingmatrix.com/articles/singhal/index.html.

Snow, C.E., Burns, M.S. \& Griffin, P. (1998). Preventing reading difficulties in young children. National Academy Press, Washington DC. Retrieved from www.nap.edu/readingroom/books/reading/

Song, M. (1998). Teaching reading strategies in and ongoing EFL university reading classroom. Asian Journal of English Language Teaching, 8, 4154.

Stake, R.E. (1995). The Art of Case Study Research. Thousand Oaks, CA: Sage Publications.

Stanovich, K.E. (1980). Toward an Interactive-Compensatory Model of Individual Differences in the Development of Reading Fluency. Reading Research Quarterly, 16(1), 32-71.

Statistics New Zealand. (n.d.). School leavers with no qualifications, 19912000. Retrieved from

http://www.stats.govt.nz/browse_for_stats/education_and_training/secon dary_education/school-leavers-with-no-qualifications/school-leavers-noqualifications.aspx

Strucker, J. \& Davidson, R. (2003). Adult Reading Components Study (ARCS). An NCSALL Research Brief, National Centre for the Study of Adult Learning and Literacy. Retrieved from http://www.ncsall.net/fileadmin/resources/research/brief_strucker2.pdf .

Sugirin. (1999, July). Studying the academic reading comprehension process: responding to methodological concerns. A paper presented at the HERDSA Annual International Conference, in Melbourne, Australia.

Sullivan, J.H. (2002). The Second Language Educators' challenge: Learning about literacy. In J.H. Sullivan (Ed.), Literacy and the Second Language Learner. Volume 1 of Research in Second Language Learning. Greenwich, CT: Information Age Publishing.

Szmerecsányi, B.M. (2004). On operationalizing syntactic complexity. JADT 2004: 7es Journées internationals d'Analyse statistique des Données 
Textuelles, 1032-1038. Retrieved from http://citeseerx.ist.psu.edu/viewdoc/summary?doi=10.1.1.109.3174.

Tabachnick, R. \& Bloch, M. (1995). Learning in and out of school: Critical perspectives on the theory of cultural compatibility. In B. Swadener \& S. Lubeck (Eds.), Children and families "at promise": Deconstructing the discourse of risk (pp. 187 - 209). Albany, NY: State University of New York.

Taillefer, G. (2005). Reading for academic purposes: the literacy practices of British, French and Spanish Law and Economics students as background for study abroad. Journal of Research in Reading, 28(4), 435-51.

Taylor, B.M. (1980). Children's memory for expository texts after reading. Reading Research Quarterly, 15 (3), 399-411.

Te Arapiki Ako, Literacy and Numeracy for Adults. (n.d.) Assessment for learning. Retrieved from http://literacyandnumeracyforadults.com/Assessment-forLearning2/Literacy-and-Numeracy-for-Adults-Assessment-Tool.

Tertiary Education Commission (TEC). (n.d.). Learning Progressions Background. Retrieved from http://www.tec.govt.nz/upload/downloads/Learning-progressionsbackground.pdf

Teemant, A., Bernhardt, E. \& Roderiguez-Munoz, M. (1997). Collaborating with content-area teachers: what we need to share. In M.A. Snow \& D.M. Brinton (Eds.), The content-based classroom: Perspectives on integrating language and content (pp. 311-318). White Plains, NY: Addison Wesley Longman.

Thistlethwaite, L.L. (1990). Critical reading for at-risk students. Journal of Reading, 33(8), 586-593.

Tinto, V. (2004, April). Access without support is not opportunity: Rethinking the first year of college for low-income students. A paper presented at the Annual Conference of the American Association of Collegiate Registrars and Admissions Officers. Las Vegas, Nevada. Retrieved from http://pathways.syr.edu/documents/2004AACRAOSpeech.pdf.

Tobias, S. (1995). Interest and metacognitive word knowledge. Journal of Educational Psychology, 87(3), 399-405.

Trabasso, T. \& Bouchard, E. (2002). Teaching readers how to comprehend text strategically. In C. C. Block \& M. Pressley (Eds.), Comprehension instruction: Research-based best practices (pp. 176-200). New York, NY: Guilford Press.

Trigwell, K. \& Ashwin, P. (2006). An exploratory study of situated conceptions of learning and learning environments. Higher Education, 51, 243-258. 
Urquhart, A.H., \& Weir, C.J. (1998). Reading in a second language: process, product and practice. London, England: Longman.

Vacca, R.T. (2002). Making a difference in adolescents' school lives: Visible and invisible aspects of content area reading. In A.E. Farstrup \& S.J. Samuels (Eds.), What research has to say about Reading Instruction (pp.184-204). Newark, DE: International Reading Association.

Van den Broek, P., Rapp, D.N. \& Kendeou, P. (2005). Integrating memorybased and constructionist processes in accounts of reading comprehension. Discourse Processes, 39(2\&3), 299-316.

van Dijk, T.A. \& Kintsch, W. (1983). Strategies of discourse comprehension. NY: Academic Press.

Van Steensil, R. (2006). Relations between socio-cultural factors, the home literacy environment and children's literacy development in the first years of primary education. Journal of Research in Reading, 29(4), 367382.

Vellutino, F.R., Tunmer, W.E., Jaccard, J.J., \& Chen, R. (2007). Components of reading ability: Multivariate evidence for a convergent skills model of reading development. Scientific Studies of Reading, 11(1), 3-32.

Wagemaker, H. (1993). Achievement in reading literacy: New Zealand's performance in a national and international context. Wellington, New Zealand: Research Section, Ministry of Education.

Wallace, C. (1986). Learning to read in a multicultural society. The social context of second language literacy. Oxford, England: Pergamon Press.

Wallace, C. (1992). Critical language awareness in the EFL classroom. In N. Fairclough (Ed.), Critical Language Awareness (pp. 59-92). London, England, Longman.

Wallace, C. (1995). 'Reading with a suspicious eye': Critical reading in the foreign language classroom. In B. Seidlhofer \& G. Cook (Eds.), Principles and practice in applied linguistics: Studies in honour of Henry Widowson (pp.335-348). Oxford, England: Oxford University Press.

Wallace, C. (2003). Critical reading in language education. Hampshire, England: Palgrave Macmillan.

Ward, A, \& Beach, S.A. (2007). Apprenticeships in critical literacy: Conversations with pre-service teachers. Policy and Practice in Education, 13(1,2), 8-24.

Warschauer, M. (1997). A sociocultural approach to literacy and its significance for CALL. In K. Murphy-Judy \& R. Sanders (Eds.), Nexus: The convergence of research \& teaching through new information technologies (pp. 88-97). Durham, NC: University of North Carolina. 
Weir, C.J., Yang, H. \& Jin, Y. (2000). An empirical investigation of the componentiality of the L2 Reading in English for academic purposes. In M. Milanovic (Ed.), Studies in Language Testing, 12. Cambridge, England: Cambridge University Press.

Wensvoort, M. (2010). Pasifika peoples in tertiary education in New Zealand: 2008. Wellington, New Zealand: Tertiary Sector Performance Analysis and Reporting Division, Ministry of Education, New Zealand.

Retrieved from

http://www.educationcounts.govt.nz/publications/tertiary_education/367 69/71683.

Wensvoort, M. (2010). 2009 Tertiary Education Enrolments. Wellington, New Zealand: Tertiary Sector Performance Analysis and Reporting Division, Ministry of Education, New Zealand. Retrieved from http://www.educationcounts.govt.nz/publications/tertiary_education/753 61.

White, H.L. (2004). Nursing instructors must also teach reading and study skills. Reading Improvement,4(1), 38- 51.

Wilson, K. (2003). A social constructivist approach to teaching reading: Turning the rhetoric into reality. Paper presented at the 16th Educational Conference, Melbourne, Australia. Retrieved from http://www.englishaustralia.com.au/ea_conference03/proceedings/pdf/0 37F_Wilson.pdf.

Wilson, K., Devereux, L., Macken-Horarik, M. \& Trimmingham-Jack, C. (2004). Reading reading: How students learn to (dis)engage with critical reading. Retrieved from http://www.herdsa.org.au/conference2004/contributions/RPapers/P048rj t.pdf .

Wilson, K. (2009). Reading in the margins: EAP reading pedagogies and their critical, postcritical potential. Retrieved from http://epress.lib.uts.edu.au/dspace/handle/2100/839.

Xue, G. \& Nation, I.S.P. (1984). A university word list. Language Learning and Communication, 3, 215-229.

Yamashita, J. (2002). Mutual compensation between L1 reading ability and L2 language proficiency in L2 reading comprehension. Journal of Research in Reading, 25(1), 81-95.

Yang, L. \& Wilson, K. (2006). Second language classroom reading: A social constructivist approach. The Reading Matrix, 6(3), 364-372.

Zhang, Z. (1994, November). The Development and Use of a Reading Strategy Inventory for ESL College Students. A Paper resented at MidSouth Educational Research Association in Nashville, Tennessee. Retrieved from http://www.eric.ed.gov/PDFS/ED382920.pdf. 
